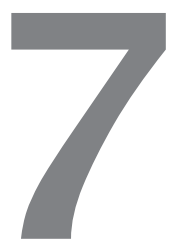

\title{
Investigação em estruturas reais
}

No início deste trabalho foi mencionado que muitas estruturas do mundo da engenharia civil podem ser sensíveis aos efeitos da não-linearidade geométrica. Mencionou-se que eram de especial importância para a investigação desses efeitos as estruturas usadas no sistema de transmissão do sinal de telefonia móvel celular.

A razão desse particular interesse deveu-se ao fato de que o Brasil, a partir de 1998, havia promovido uma reestruturação do seu sistema de telecomunicações para estimular o crescimento e a universalização dos serviços de telefonia. Com isso, favoreceu a implantação, em todo o território brasileiro, de milhares de estações para a transmissão do sinal de telefonia móvel celular. Para tanto, os opera- 
dores privados, valeram-se de estruturas compostas, em muitos casos, apenas de postes em balanço de elevada esbelteza e, por conseguinte, sensíveis às ações dinâmicas do vento.

Como a implantação do sistema de telefonia móvel deu-se em um ritmo acelerado, não houve tempo hábil para que os profissionais envolvidos nos projetos dessas estruturas adaptassem seus modelos de cálculo e, portanto, mantiveram em uso aqueles com os quais estavam mais familiriazados. Nesse sentido, os projetos desenvolvidos para construção dos postes de telecomunicações estavam baseados no processo de cálculo previsto no item 4 da NBR 6123/88, denominado, neste trabalho, de modelo estático, e descrito na seção 6.1.

O presente capítulo visa avaliar dois aspectos relativos aos postes de telecomunicações, que são:

- a diferença produzida entre o modelo estático de cálculo para a ação do vento, usado no dimensionamento das estruturas, e os demais modelos presentes na NBR 6123/88; e

- a influência que a rigidez geométrica exerce no cálculo da frequência e na, consequente, resposta dinâmica devida à turbulência atmosférica.

Para isso foram selecionados cinco postes de telecomunicações. Os dados relativos à geometria das estruturas e os parâmetros para o cálculo da ação do vento foram gentilmente cedidos pela RM Engenharia, sediada na cidade de São Paulo-SP. Informações não autorizadas foram intencionalmente omitidas.

O aço foi considerado submetido a tensões dentro da região elástica de deformação. Quando necessário, o módulo de elasticidade do concreto foi calculado usando-se a expressão da NBR 6118/04 - Projeto de Estruturas de Concreto Armado, seguindo a expressão $\mathrm{E}_{\mathrm{ci}}=5600 \sqrt{\mathrm{f}_{\mathrm{ck}}}$, onde $\mathrm{f}_{\mathrm{ck}}$ é a resistência característica à compressão do concreto em $\mathrm{MPa}$. O módulo de elasticidade secante do concreto, recomendado para as análises elásticas de projeto, é definido pela NBR 6118/04 como $\mathrm{E}_{\mathrm{cs}}=0,85 \mathrm{E}_{\mathrm{ci}}$. O módulo de elasticidade e o peso específico do aço, adotados nas análises das estruturas reais, de $205 \mathrm{GPa}$ e $77 \mathrm{kN} / \mathrm{m}^{3}$, foram os recomendados pela NBR 8800/96 - Projeto e execução de estruturas de aço de edifícios. A densidade corresponde ao peso específico do aço é, portanto, $7850 \mathrm{~kg} / \mathrm{m}^{3}$. Mes- 
mo já tendo sido mencionadas anteriormente, as características dos materiais também foram indicadas em cada análise.

$\mathrm{Na}$ determinação dos esforços devidos ao vento foram desenvolvidos cinco processos de cálculo. Inicialmente foi utilizado o método para a determinação exclusiva da ação do vento como força estática (modelo estático), previsto no item 4 da NBR 6123/88 - Forças devidas ao vento em edificações.

No segundo e no terceiro processos foram usados o método contínuo simplificado (modelo dinâmico simplificado), estipulado no item 9.3.1 da NBR 6123/88, sendo a frequência obtida sob condições lineares e não-lineares. Nos outros dois modelos, a resposta dinâmica da estrutura foi obtida utilizando-se o modelo dinâmico discreto (análise dinâmica discreta), conforme preconizado no item 9.3.2 da NBR 6123/88, também utilizando-se modelos lineares e não-lineares. Os modelos simplificado e discreto estão previstos no capítulo 9 - Cálculo da resposta dinâmica na direção do vento médio, da NBR 6123/88. Todos os modelos para o cálculo da ação do vento, mencionados anteriormente, foram apresentados no capítulo 6 do presente trabalho.

Os processos de cálculos dinâmicos lineares foram desenvolvidos buscando-se o máximo de apoio nas prescrições da NBR 6123/88. Já os métodos não-lineares foram desenvolvidos levando em conta a influência do esforço normal no cálculo das frequências e nas formas modais naturais de vibração das estruturas.

A discretização da massa e as formas modais das estruturas foram obtidas pelos modelos elaborados em Elementos Finitos, no programa SAP2000.

Os comentários acerca do emprego do Método dos Elementos Finitos foram feitos no capítulo 3. No entanto, o emprego do método proposto nesta Tese para o cálculo da frequência fundamental requer que sejam feitos comentários adicionais neste momento. Como as estruturas possuem geometria e propriedades variando com a altura, o emprego do método deve ser feito por trechos, sendo suas integrais resolvidas nos limites estabelecidos para cada intervalo.

Para o emprego correto do método, as propriedades generalizadas como as massas e as rigidezes também devem ser calculadas para 
cada parte da estrutura. Cuidado especial deve ser tomado ao serem calculadas as rigidezes geométricas, pois cada parcela deve levar em conta a força normal distribuída no respectivo intervalo e os esforços que atuam nos segmentos superiores.

De um modo geral, o emprego do método proposto nesta pesquisa foi dividido nas seguintes etapas: definição dos dados e geometria, cálculo da massa generalizada, determinação da rigidez generalizada e cálculo da frequência. Chama-se a atenção para o fato de que nenhum fator de majoração dos esforços ou minoração das resistências dos materiais foi adotado.

\subsection{ESTRUTURA 1 - POSTE METÁLICO DE 48 M}

\subsubsection{Dados e geometria}

Trata-se de um poste metálico destinado ao suporte do sistema irradiante do sinal de telefonia móvel celular. A estrutura possui 48 metros de altura e seção transversal circular vazada de diâmetro externo (申ext) e espessura (e) variáveis.

A Tabela 7.1 e a Figura 7.1 apresentam as propriedades da estrutura e a discretização do modelo. Na Figura 7.2 podem ser vistas fotografias da estrutura.

$\mathrm{O}$ índice de esbeltez da estrutura é $\lambda=310$. Cabe mencionar que o comitê de revisão da NBR 8800/96 - Projeto e execução de estruturas de aço de edifícios, na proposta de revisão de abril de 2006, sugere que a esbeltez das barras comprimidas não deve ser superior a 200 .

A Tabela 7.2 apresenta os parâmetros estruturais e dos dispositivos existentes para o cálculo da ação do vento. 


\begin{tabular}{|c|c|c|c|c|c|c|c|c|c|c|c|c|}
\hline ๑ं & छ్ & $\begin{array}{l}\circ \\
\infty \\
0^{\circ}\end{array}$ & $\begin{array}{l}\circ \\
\infty \\
0^{\circ}\end{array}$ & $\begin{array}{l}\circ \\
\infty \\
0^{\circ}\end{array}$ & $\begin{array}{l}\infty \\
\infty \\
0^{\circ}\end{array}$ & $\begin{array}{l}\infty \\
\infty \\
0^{\prime}\end{array}$ & $\begin{array}{l}\circ \\
\infty \\
0^{\prime}\end{array}$ & $\begin{array}{l}\circ \\
\infty \\
\circ\end{array}$ & $\begin{array}{l}\infty \\
\infty \\
0^{\prime}\end{array}$ & $\begin{array}{l}\circ \\
\infty \\
0^{\circ}\end{array}$ & $\begin{array}{l}\circ \\
\infty \\
0^{\prime}\end{array}$ & $\begin{array}{l}\circ \\
\infty \\
0^{\circ}\end{array}$ \\
\hline$\underset{\longleftarrow}{\stackrel{x}{x}}$ & छ्छ & $\begin{array}{l}\text { மீ } \\
\text { ஸ̃ }\end{array}$ & $\begin{array}{l}\check{\sigma} \\
\stackrel{0}{0}\end{array}$ & 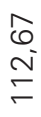 & $\begin{array}{l}\underset{N}{N} \\
\stackrel{\sim}{\sim} \\
\sim\end{array}$ & $\frac{\infty}{N}$ & $\begin{array}{l}m \\
\text { m } \\
\stackrel{m}{n}\end{array}$ & 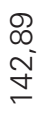 & 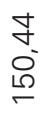 & $\begin{array}{l}\circ \\
\text { 우 } \\
\stackrel{0}{\circ}\end{array}$ & 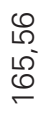 & $\underset{\check{N}}{\check{\sigma}}$ \\
\hline$\stackrel{\text { İ }}{\stackrel{0}{0}}$ & छ્ & $\begin{array}{l}\circ \\
0 \\
0 \\
0\end{array}$ & $\begin{array}{l}8 \\
\text { - }\end{array}$ & $\begin{array}{l}8 \\
\infty \\
\infty\end{array}$ & ○ㅇ & $\begin{array}{l}8 \\
0\end{array}$ & $\begin{array}{l}8 \\
8 \\
10\end{array}$ & $\underset{\forall}{\text { ৪ }}$ & $\begin{array}{l}\text { ○ } \\
\text { ले }\end{array}$ & $\begin{array}{l}8 \\
\text { ○ }\end{array}$ & $\stackrel{8}{8}$ & $\begin{array}{l}8 \\
0 \\
0\end{array}$ \\
\hline ๑ं & छ્ & $\begin{array}{l}\circ \\
\infty \\
0\end{array}$ & $\begin{array}{l}\circ \\
\infty \\
0\end{array}$ & $\begin{array}{l}0 \\
\infty \\
0\end{array}$ & $\begin{array}{l}0 \\
\infty \\
0\end{array}$ & $\begin{array}{l}\circ \\
\infty \\
0\end{array}$ & $\begin{array}{l}\circ \\
\infty \\
0\end{array}$ & $\begin{array}{l}8 \\
\infty \\
0\end{array}$ & $\begin{array}{l}0 \\
\infty \\
0 \\
0\end{array}$ & $\begin{array}{l}0 \\
\infty \\
0 \\
0\end{array}$ & $\begin{array}{l}0 \\
\infty \\
0 \\
0\end{array}$ & \\
\hline 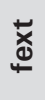 & छ્ & $\begin{array}{l}\text { ○ } \\
\text { ○' }\end{array}$ & $\begin{array}{l}\text { 8. } \\
\text { ه' }\end{array}$ & $\begin{array}{l}\text { ৪ } \\
\text { ه' }\end{array}$ & $\begin{array}{l}\text { ○ } \\
\text { ○' }\end{array}$ & $\begin{array}{l}\text { ৪ } \\
\text { ᄋ' }\end{array}$ & $\begin{array}{l}\text { ৪ } \\
\text { ᄋ' }\end{array}$ & $\begin{array}{l}\text { ৪ } \\
\text { ᄋ' }\end{array}$ & $\begin{array}{l}\text { ○ } \\
\text { ○் }\end{array}$ & $\begin{array}{l}\text { ○ } \\
\text { ○' }\end{array}$ & $\begin{array}{l}8 \\
\text { ○' } \\
\text { هं }\end{array}$ & \\
\hline ث̊ & छ & $\begin{array}{l}\text { ○ } \\
\text { ○ }\end{array}$ & $\begin{array}{l}\text { ㅇ } \\
\text { த் }\end{array}$ & $\begin{array}{l}8 \\
\infty \\
\infty \\
-\end{array}$ & $\begin{array}{l}8 \\
\stackrel{-}{-}\end{array}$ & $\begin{array}{l}8 \\
0^{\circ} \\
-\end{array}$ & $\begin{array}{l}8 \\
10 \\
\leftarrow\end{array}$ & $\begin{array}{l}8 \\
\text { ○ } \\
\stackrel{-}{-}\end{array}$ & $\begin{array}{l}8 \\
\text { mे }\end{array}$ & $\begin{array}{l}8 \\
\stackrel{0}{\sim} \\
\sim\end{array}$ & $\begin{array}{l}8 \\
\text { - } \\
=\end{array}$ & \\
\hline ๑ं & छ्ट & $\begin{array}{l}\circ \\
\infty \\
0 \\
0\end{array}$ & $\begin{array}{l}\circ \\
\infty \\
\circ\end{array}$ & $\begin{array}{l}\circ \\
\infty \\
0 \\
0\end{array}$ & $\begin{array}{l}\infty \\
\infty \\
\circ\end{array}$ & $\begin{array}{l}0 \\
\infty \\
0\end{array}$ & $\begin{array}{l}0 \\
\infty \\
0\end{array}$ & $\begin{array}{l}\bigcirc \\
\infty \\
0^{\circ}\end{array}$ & $\begin{array}{l}0 \\
\infty \\
0\end{array}$ & $\begin{array}{l}\circ \\
\infty \\
0 \\
0\end{array}$ & $\begin{array}{l}0 \\
\infty \\
0^{-}\end{array}$ & \\
\hline 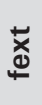 & छ् & $\begin{array}{l}8 \\
0 \\
\infty \\
\infty\end{array}$ & $\begin{array}{l}\text { ○ } \\
\circ \\
\infty\end{array}$ & $\begin{array}{l}8 \\
0 \\
\infty \\
\infty\end{array}$ & $\begin{array}{l}8 \\
8 \\
\infty \\
\infty\end{array}$ & $\begin{array}{l}8 \\
\circ \\
\infty\end{array}$ & $\begin{array}{l}8 \\
\circ \\
\infty\end{array}$ & $\begin{array}{l}\text { 8 } \\
\text { ○் }\end{array}$ & $\begin{array}{l}\text { 8. } \\
\text { ○் }\end{array}$ & $\begin{array}{l}\text { ○ } \\
\text { ○் }\end{array}$ & $\begin{array}{l}\text { ○ } \\
\text { ○் }\end{array}$ & \\
\hline ঙ্ঠ & छ్ & $\begin{array}{l}\text { ○ } \\
\text { оे }\end{array}$ & $\begin{array}{l}\text { ○ } \\
\text { D }\end{array}$ & $\begin{array}{l}\text { ○ } \\
\infty \\
\stackrel{0}{\sim}\end{array}$ & 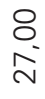 & $\begin{array}{l}8 \\
0 \\
\stackrel{0}{ }\end{array}$ & $\begin{array}{l}8 \\
\stackrel{\circ}{\circ} \\
\sim\end{array}$ & $\begin{array}{l}8 \\
\stackrel{-}{\sim} \\
\text { D }\end{array}$ & $\begin{array}{l}\text { ৪ } \\
\text { Nं }\end{array}$ & $\begin{array}{l}8 \\
\text { ㄱ } \\
\text { N }\end{array}$ & $\begin{array}{l}\text { ৪ } \\
\text { ড }\end{array}$ & \\
\hline •ं & छ् & $\begin{array}{l}\infty \\
\stackrel{\infty}{+} \\
0^{\circ}\end{array}$ & $\begin{array}{l}\infty \\
\stackrel{\infty}{+} \\
0^{\prime}\end{array}$ & $\begin{array}{l}\infty \\
\stackrel{\infty}{+} \\
0^{\circ}\end{array}$ & $\begin{array}{l}\circ \\
\infty \\
0^{\circ}\end{array}$ & $\begin{array}{l}\circ \\
\infty \\
0^{\circ}\end{array}$ & $\begin{array}{l}0 \\
\infty \\
0^{-}\end{array}$ & $\begin{array}{l}\circ \\
\infty \\
0^{\circ}\end{array}$ & $\begin{array}{l}\circ \\
\infty \\
0^{\circ}\end{array}$ & $\begin{array}{l}\circ \\
\infty \\
0^{\circ}\end{array}$ & $\begin{array}{l}\infty \\
\infty \\
0^{-}\end{array}$ & \\
\hline 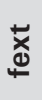 & छ्ट & $\begin{array}{l}\text { ৫ } \\
\text { ○' }\end{array}$ & 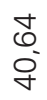 & $\begin{array}{l}\text { ৫ } \\
\text { ○' }\end{array}$ & $\begin{array}{l}8 \\
0 \\
0\end{array}$ & $\begin{array}{l}8 \\
\varnothing \\
0 \\
\end{array}$ & $\begin{array}{l}8 \\
\varnothing \\
0 \\
\end{array}$ & $\begin{array}{l}\text { ○ } \\
\text { ○ } \\
\text { - }\end{array}$ & $\begin{array}{l}\text { O } \\
\text { ' } \\
\end{array}$ & $\begin{array}{l}\text { ○ } \\
\circ \\
\end{array}$ & $\begin{array}{l}8 \\
\circ \\
\infty\end{array}$ & \\
\hline ڤั) & $\xi$ & $\begin{array}{l}\text { ○ } \\
\text { o- } \\
\text { ণ }\end{array}$ & $\begin{array}{l}\text { ○ } \\
\text { ó }\end{array}$ & $\begin{array}{l}\text { ৪ } \\
\text { J }\end{array}$ & $\begin{array}{l}\stackrel{8}{0} \\
\text { N }\end{array}$ & $\begin{array}{l}\text { ○ } \\
\text { ᄋ́ }\end{array}$ & $\begin{array}{l}\text { ○ } \\
\text { on }^{0}\end{array}$ & $\begin{array}{l}8 \\
\text { @' }\end{array}$ & $\begin{array}{l}\text { ৪ } \\
\text { ले }\end{array}$ & $\begin{array}{l}\text { O } \\
\text { Nं }\end{array}$ & $\frac{8}{\text { - }}$ & \\
\hline
\end{tabular}


Tabela 7.2 - Estrutura 1: características dos dispositivos.

\begin{tabular}{|c|c|c|c|c|}
\hline Dispositivo & $\begin{array}{c}\text { Área } \\
\text { frontal }\end{array}$ & Ca & Cota & $\begin{array}{c}\text { Peso específico, Peso } \\
\text { distribuído ou Peso }\end{array}$ \\
\hline Poste & Variável & 0,6 & de 0 a $48 \mathrm{~m}$ & $7850 \mathrm{kN} / \mathrm{m}^{3}$ \\
\hline Escada & $0,05 \mathrm{~m}^{2} / \mathrm{m}$ & 2,0 & de 0 a $48 \mathrm{~m}$ & $0,15 \mathrm{kN} / \mathrm{m}$ \\
\hline Cabos & $0,15 \mathrm{~m}^{2} / \mathrm{m}$ & 1,2 & de 0 a $48 \mathrm{~m}$ & $0,25 \mathrm{kN} / \mathrm{m}$ \\
\hline $\begin{array}{c}\text { Antenas e } \\
\text { suportes }\end{array}$ & $1,1 \mathrm{~m}^{2}$ & 1,0 & $48 \mathrm{~m}$ & $3,36 \mathrm{kN}$ \\
\hline
\end{tabular}

$(\mathrm{Ca}=$ Coeficiente de arrasto $)$
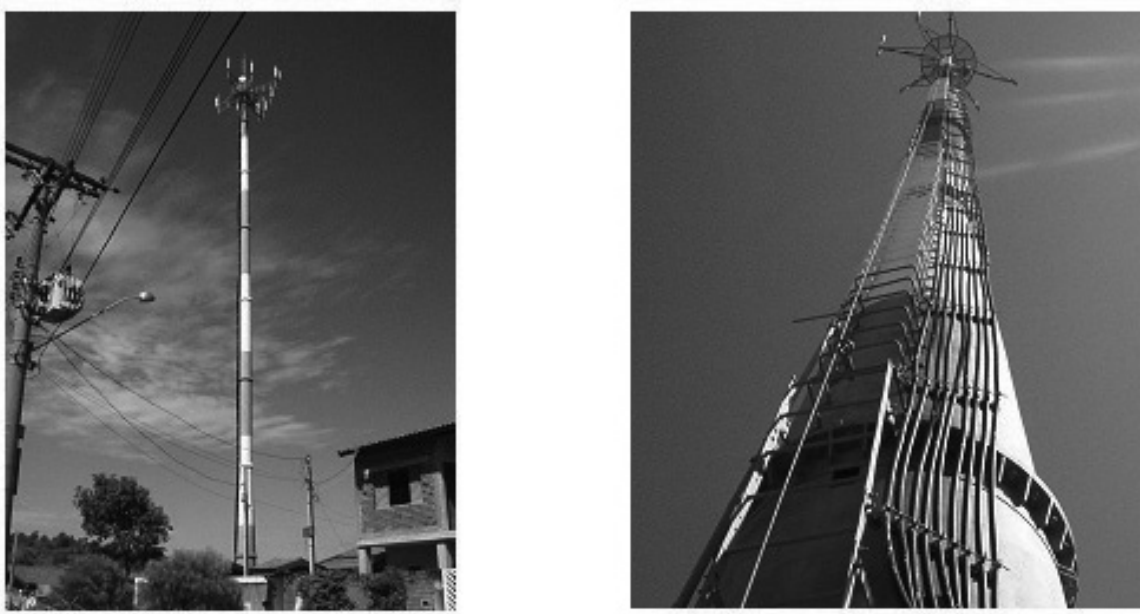

Figura 7.1 - Estrutura 1: Fotografias. 


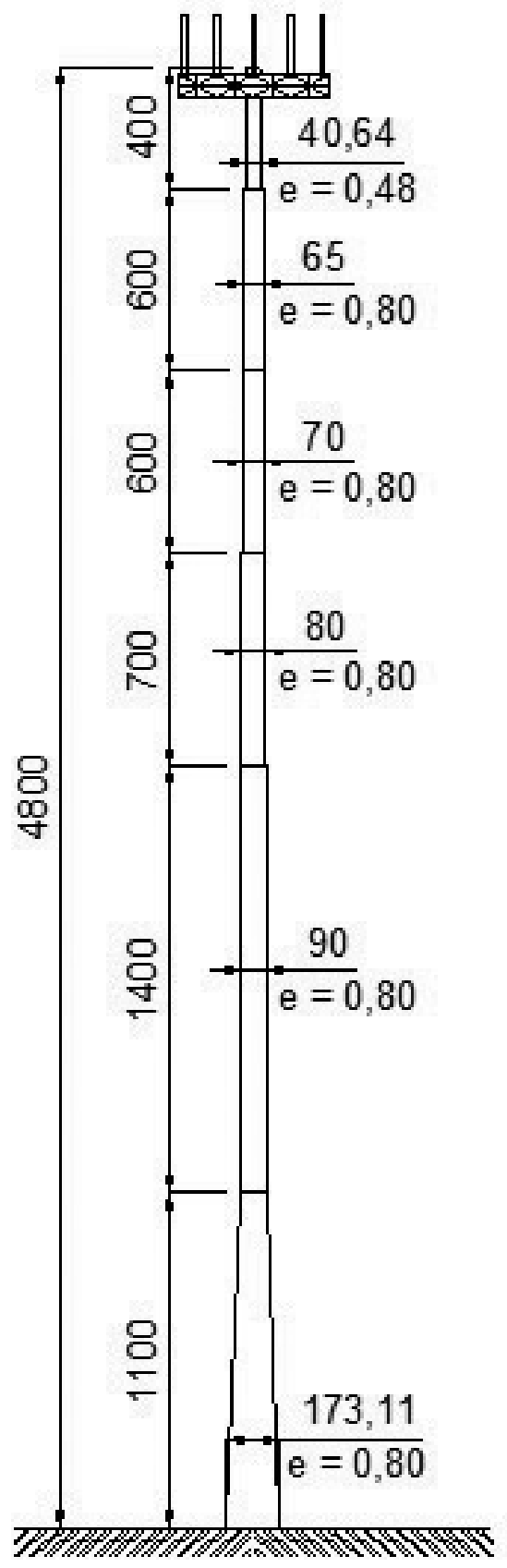

Figura 7.2 - Estrutura 1: Geometria - Medidas em centímetro. 


\subsubsection{Modelagem por Elementos Finitos}

A estrutura foi modelada utilizando-se elementos de barra com seções transversais constantes e variáveis, conforme o caso. No modelo foram atribuídas as forças descritas na Tabela 7.2, com as correspondentes massas.

A Figura 7.3 apresenta o modelo tridimensional disponibilizado pelo programa e a discretização da estrutura construída com 40 elementos de barra.

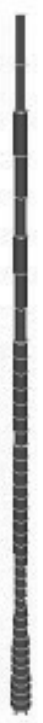

(a) $3 \mathrm{D}$

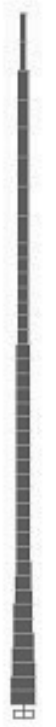

(b) Vista lateral

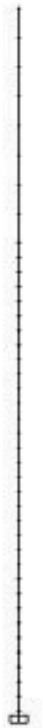

(c) Discretização

Figura 7.3 - Estrutura 1: modelo por Elementos Finitos.

Os modos de vibração e as frequências obtidas pelo Método dos Elementos Finitos são as constantes na Figura 7.4. 


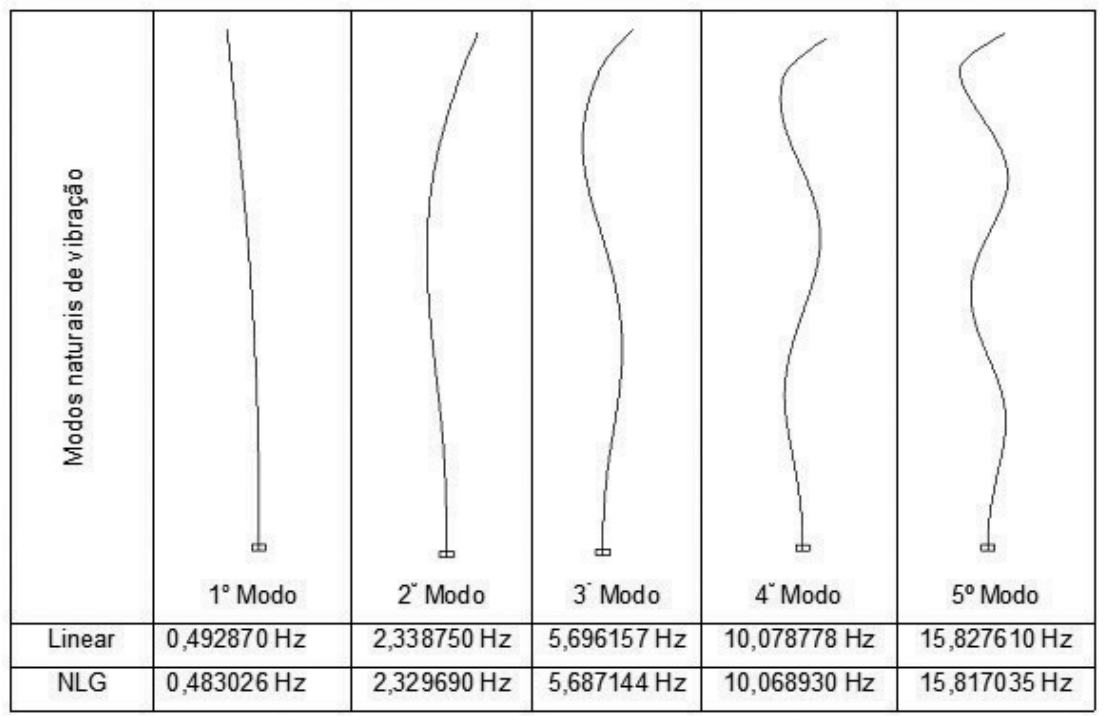

(NLG = Não-Linearidade Geométrica)

Figura 7.4 - Estrutura 1: modos naturais de vibração.

\subsubsection{Aplicação do método proposto}

\subsubsection{Definição dos parâmetros}

Os dados utilizados na aplicação do método proposto foram os seguintes:

- módulo de elasticidade: $\mathrm{E}=205 \mathrm{GPa}$,

- $\quad$ densidade do aço: $\rho=7850 \mathrm{~kg} / \mathrm{m}^{3}$,

- $\quad$ massa concentrada no topo: $\mathrm{m}_{0}=342,40 \mathrm{~kg}$;

- $\quad$ massa distribuída por unidade de altura: $\mathrm{m}_{\mathrm{e}}=40 \mathrm{~kg} / \mathrm{m}$.

As ordenadas correspondentes às alturas na estrutura e as propriedades geométricas das seções transversais, dos respectivos trechos, são dadas por: 
Na base, quando $\mathrm{x}=0$, tem-se: $\mathrm{D}_{1}=173,11 \mathrm{~cm}, \mathrm{e}_{1}=0,80 \mathrm{~cm}$, $\mathrm{d}_{1}=\mathrm{D}_{1}-2 \mathrm{e}_{1}, \mathrm{~A}_{1}=\frac{\pi}{4}\left(\mathrm{D}_{1}^{2}-\mathrm{d}_{1}^{2}\right), \mathrm{I}_{1}=\frac{\pi}{64}\left(\mathrm{D}_{1}^{4}-\mathrm{d}_{1}^{4}\right)$.

No segmento subsequente, de propriedades variáveis, tem-se: $\mathrm{D}(\mathrm{x})=\frac{\mathrm{D}_{2}-\mathrm{D}_{1}}{\mathrm{~L}_{1}} \mathrm{x}+\mathrm{D}_{1}, \mathrm{~d}(\mathrm{x})=\mathrm{D}(\mathrm{x})-2 \mathrm{e}_{1}$, $\mathrm{A}(\mathrm{x})=\frac{\pi}{4}\left(\mathrm{D}(\mathrm{x})^{2}-\mathrm{d}_{1}(\mathrm{x})^{2}\right), \mathrm{I}(\mathrm{x})=\frac{\pi}{64}\left(\mathrm{D}(\mathrm{x})^{4}-\mathrm{d}(\mathrm{x})^{4}\right)$.

Na ordenada $L_{1}=11 \mathrm{~m}$, define-se: $\mathrm{D}_{2}=90,00 \mathrm{~cm}$, $\mathrm{e}_{2}=0,80 \mathrm{~cm}, \mathrm{~d}_{2}=\mathrm{D}_{2}-2 \mathrm{e}_{2}, \mathrm{~A}_{2}=\frac{\pi}{4}\left(\mathrm{D}_{2}^{2}-\mathrm{d}_{2}^{2}\right)$, $\mathrm{I}_{2}=\frac{\pi}{64}\left(\mathrm{D}_{2}^{4}-\mathrm{d}_{2}^{4}\right)$

Em $\mathrm{L}_{2}=25,00 \mathrm{~m}$, tem-se: $\mathrm{D}_{3}=80,00 \mathrm{~cm}, \mathrm{e}_{3}=0,80 \mathrm{~cm}$, $\mathrm{d}_{3}=\mathrm{D}_{3}-2 \mathrm{e}_{3}, \mathrm{~A}_{3}=\frac{\pi}{4}\left(\mathrm{D}_{3}{ }^{2}-\mathrm{d}_{3}{ }^{2}\right), \mathrm{I}_{3}=\frac{\pi}{64}\left(\mathrm{D}_{3}{ }^{4}-\mathrm{d}_{3}^{4}\right)$.

Em $\mathrm{L}_{3}=32,00 \mathrm{~m}$, tem-se: $\mathrm{D}_{4}=70,00 \mathrm{~cm}, \mathrm{e}_{4}=0,80 \mathrm{~cm}$, $\mathrm{d}_{4}=\mathrm{D}_{4}-2 \mathrm{e}_{4}, \mathrm{~A}_{4}=\frac{\pi}{4}\left(\mathrm{D}_{4}^{2}-\mathrm{d}_{4}^{2}\right), \mathrm{I}_{4}=\frac{\pi}{64}\left(\mathrm{D}_{4}^{4}-\mathrm{d}_{4}^{4}\right)$.

Em $\mathrm{L}_{4}=38,00 \mathrm{~m}$, tem-se: $\mathrm{D}_{5}=65,00 \mathrm{~cm}, \mathrm{e}_{5}=0,80 \mathrm{~cm}$, $\mathrm{d}_{5}=\mathrm{D}_{5}-2 \mathrm{e}_{5}, \mathrm{~A}_{5}=\frac{\pi}{4}\left(\mathrm{D}_{5}^{2}-\mathrm{d}_{5}^{2}\right), \mathrm{I}_{5}=\frac{\pi}{64}\left(\mathrm{D}_{5}^{4}-\mathrm{d}_{5}^{4}\right)$.

Para $\mathrm{L}_{5}=44,00 \mathrm{~m} \mathrm{e} \mathrm{L}_{6}=48,00 \mathrm{~m}$, tem-se: $\mathrm{D}_{6}=40,64 \mathrm{~cm}$, $\mathrm{e}_{5}=0,48 \mathrm{~cm}, \mathrm{~d}_{6}=\mathrm{D}_{6}-2 \mathrm{e}_{6}, \mathrm{~A}_{6}=\frac{\pi}{4}\left(\mathrm{D}_{6}^{2}-\mathrm{d}_{6}{ }^{2}\right)$, $\mathrm{I}_{5}=\frac{\pi}{64}\left(\mathrm{D}_{5}^{4}-\mathrm{d}_{5}^{4}\right)$ 


\subsubsection{Cálculo da massa generalizada}

Os subíndices em números romanos, introduzidos a partir deste ponto, visam evitar redundância de notação nas expressões. A massa generalizada foi obtida por meio das integrais dispostas a seguir.

Para o primeiro segmento:

$$
\mathrm{m}_{1}=\int_{0}^{\mathrm{L}_{1}} \mathrm{~m}_{\mathrm{I}}(\mathrm{x}) \phi(\mathrm{x})^{2} \mathrm{dx}, \text { com } \mathrm{m}_{\mathrm{I}}(\mathrm{x})=\mathrm{A}(\mathrm{x}) \rho+\mathrm{m}_{\mathrm{e}} .
$$

Para o segundo segmento:

$$
\mathrm{m}_{2}=\int_{\mathrm{L}_{1}}^{\mathrm{L}_{2}} \mathrm{~m}_{\mathrm{II}} \phi(\mathrm{x})^{2} \mathrm{dx}, \text { com } \mathrm{m}_{\mathrm{II}}=\mathrm{A}_{2} \rho+\mathrm{m}_{\mathrm{e}} .
$$

Analogamente ao segundo trecho, para os demais, pode-se escrever na forma geral

$$
m_{i}=\int_{L_{i-1}}^{L_{i}} m_{i} \phi(x)^{2} d x, \text { com } m_{i}=A_{i} \rho+m_{e} .
$$

A massa distribuída generalizada foi obtida por

$$
\mathrm{m}_{\mathrm{R}}=\sum_{\mathrm{i}=1}^{6} \mathrm{~m}_{\mathrm{i}}
$$

E a massa generalizada total por:

$$
\mathrm{M}=\mathrm{m}_{0}+\mathrm{m}_{\mathrm{R}}
$$

\subsubsection{Cálculo da rigidez generalizada}

\section{Rigidez geométrica generalizada}

Para calcular a rigidez geométrica generalizada foi preciso determinar os esforços normais relativos aos trechos definidos na geometria. Do topo para a base da estrutura os esforços normais são: 


$$
\begin{gathered}
\mathrm{F}_{0}=\mathrm{m}_{0} \mathrm{~g}, \\
\mathrm{~F}_{6}=\int_{\mathrm{L}_{5}}^{\mathrm{L}_{5}} \mathrm{~m}_{\mathrm{VI}} \mathrm{gdx}, \\
\mathrm{F}_{5}=\int_{\mathrm{L}_{4}}^{\mathrm{L}_{5}} \mathrm{~m}_{\mathrm{V}} \mathrm{gdx},
\end{gathered}
$$

e assim sucessivamente, ou seja:

$$
F_{i}=\int_{L_{i-1}}^{L_{i}} m_{i} g d x
$$

sendo que o esforço normal relativo ao primeiro segmento, que é linearmente variável, foi obtido pela seguinte expressão

$$
F_{1}=\int_{0}^{L_{1}} m_{I}(x) g d x, .
$$

A força normal generalizada $\mathrm{F}$ é então:

$$
F=\sum_{i=0}^{6} F_{i} .
$$

E as rigidezes geométricas foram calculadas pelas seguintes expressões:

$$
\begin{aligned}
& \mathrm{K}_{\mathrm{g} 6}=\int_{\mathrm{L}_{5}}^{\mathrm{L}}\left[\mathrm{F}_{0}+\mathrm{m}_{\mathrm{VI}}\left(\mathrm{L}_{6}-\mathrm{x}\right) \mathrm{g}\left(\frac{\mathrm{d}}{\mathrm{dx}} \phi(\mathrm{x})\right)^{2}\right], \\
& \mathrm{K}_{\mathrm{g} 5}=\int_{\mathrm{L}_{4}}^{\mathrm{L}_{5}}\left[\mathrm{~F}_{0}+\mathrm{F}_{6}+\mathrm{m}_{\mathrm{V}}\left(\mathrm{L}_{5}-\mathrm{x}\right) \mathrm{g}\left(\frac{\mathrm{d}}{\mathrm{dx}} \phi(\mathrm{x})\right)^{2}\right], \\
& \mathrm{K}_{\mathrm{g} 4}=\int_{\mathrm{L}_{3}}^{\mathrm{L}_{4}}\left[\mathrm{~F}_{0}+\mathrm{F}_{6}+\mathrm{F}_{5}+\mathrm{m}_{\mathrm{IV}}\left(\mathrm{L}_{4}-\mathrm{x}\right) \mathrm{g}\left(\frac{\mathrm{d}}{\mathrm{dx}} \phi(\mathrm{x})\right)^{2}\right],
\end{aligned}
$$




$$
\begin{aligned}
& \mathrm{K}_{\mathrm{g} 3}=\int_{\mathrm{L}_{2}}^{\mathrm{L}_{3}}\left[\mathrm{~F}_{0}+\mathrm{F}_{6}+\mathrm{F}_{5}+\mathrm{F}_{4}+\mathrm{m}_{\mathrm{III}}\left(\mathrm{L}_{3}-\mathrm{x}\right) \mathrm{g}\left(\frac{\mathrm{d}}{\mathrm{dx}} \phi(\mathrm{x})\right)^{2}\right], \\
& \mathrm{K}_{\mathrm{g} 2}=\int_{\mathrm{L}_{1}}^{\mathrm{L}_{2}}\left[\mathrm{~F}_{0}+\mathrm{F}_{6}+\mathrm{F}_{5}+\mathrm{F}_{4}+\mathrm{F}_{3}+\mathrm{m}_{\mathrm{II}}\left(\mathrm{L}_{2}-\mathrm{x}\right) \mathrm{g}\left(\frac{\mathrm{d}}{\mathrm{dx}} \phi(\mathrm{x})\right)^{2}\right] . \\
& \mathrm{K}_{\mathrm{g} 1}=\int_{0}^{\mathrm{L}_{1}}\left[\mathrm{~F}_{0}+\mathrm{F}_{6}+\mathrm{F}_{5}+\mathrm{F}_{4}+\mathrm{F}_{3}+\mathrm{F}_{2}+\mathrm{m}_{\mathrm{I}}(\mathrm{x})\left(\mathrm{L}_{1}-\mathrm{x}\right) \mathrm{g}\left(\frac{\mathrm{d}}{\mathrm{dx}} \phi(\mathrm{x})\right)^{2}\right] .
\end{aligned}
$$

A rigidez geométrica generalizada $\mathrm{K}_{\mathrm{g}}$ da estrutura é, portanto:

$$
\mathrm{K}_{\mathrm{g}}=\sum_{\mathrm{i}=1}^{6} \mathrm{~K}_{\mathrm{gi}}
$$

\section{Rigidez elástica generalizada}

As parcelas da rigidez elástica são:

$$
\begin{gathered}
\mathrm{K}_{01}=\int_{0}^{\mathrm{L}_{1}} \mathrm{EI}(\mathrm{x})\left(\frac{\mathrm{d}^{2}}{\mathrm{dx}^{2}} \phi(\mathrm{x})\right)^{2} \mathrm{dx}, \\
\mathrm{K}_{02}=\int_{\mathrm{L}_{1}}^{\mathrm{L}_{2}} \mathrm{EI}_{2}\left(\frac{\mathrm{d}^{2}}{\mathrm{dx}^{2}} \phi(\mathrm{x})\right)^{2} \mathrm{dx},
\end{gathered}
$$

que, de forma análoga ao segundo trecho, para os demais, pode ser escrita como

$$
K_{0 i}=\int_{L_{i-1}}^{L_{i}} E I_{i}\left(\frac{d^{2}}{d x^{2}} \phi(x)\right)^{2} d x
$$


$\mathrm{E}$ a rigidez elástica generalizada $\mathrm{K}_{0}$ será dada pela soma de suas parcelas, logo:

$$
\mathrm{K}_{0}=\sum_{\mathrm{i}=1}^{6} \mathrm{~K}_{0 \mathrm{i}}
$$

\subsubsection{Cálculo da frequência}

As frequências do primeiro modo de vibração da estrutura pelo método proposto são as seguintes: modelo linear $=0,569799 \mathrm{~Hz}$, modelo não-linear $=0,562350 \mathrm{~Hz}$.

\subsubsection{Ação do vento}

\subsubsection{Forças estáticas devidas ao vento}

Os parâmetros empregados na determinação das forças estáticas devidas a ação do vento foram:

- $\quad$ fator topográfico $\mathrm{S}_{1}=1,0$;

- fator de rugosidade do terreno $\mathrm{S}_{2}$ correspondente à categoria III, classe B, com os parâmetros $p=0,105, b=0,940$ e $\mathrm{F}_{\mathrm{r}}=0,980$;

- $\quad$ fator estatístico $\mathrm{S}_{3}=1,1$;

- velocidade básica do vento $\mathrm{V}_{0}=45 \mathrm{~m} / \mathrm{s}$.

\subsubsection{Resposta dinâmica pelo modelo simplificado da NBR 6123/88}

Os parâmetros usados na determinação da resposta dinâmica pelo modelo contínuo simplificado da NBR 6123/88 foram os seguintes: largura da edificação $0,875 \mathrm{~m}$; altura da edificação $48 \mathrm{~m}$; categoria do terreno III; velocidade básica do vento e fatores estatísticos como descrito no item anterior. 
A NBR 6123/88 sugere o expoente de 1,7 para a forma modal e uma razão de amortecimento crítico de 0,008. Para o cálculo da frequência recorrer-se-á, subsidiariamente, à recomendação contida na Tabela 6.4 (Tabela 19 da NBR 6123/88), item Edifícios com estrutura de aço soldada. O inverso da expressão $0,29 \sqrt{\mathrm{h}}-0,4$, portanto, fornece $\mathrm{f}_{1}=0,621435 \mathrm{~Hz}$, um resultado distante $9 \%$ do calculado pelo método proposto neste trabalho e $22,27 \%$ do calculo efetuado pelo MEF. Com isso, relação adimensional Vp/(f $\left.f_{1} \mathrm{~L}\right)$ torna-se igual a 0,031. Adotando a taxa de amortecimento crítico z igual a 0,008 chega-se a um coeficiente de amplificação dinâmica $\xi$ de 2,406; obtido por interpolação linear.

Uma segunda avaliação, usando o modelo simplificado da NBR 6123/88, foi feita sob condições não-lineares. Esse modelo foi chamado de Modelo Simplificado Não-Linear por estar apoiado na frequência e na forma do primeiro modo de vibração do modelo não-linear. A frequência da estrutura sob não-linearidade geométrica é 0,48318 Hz. Com isso, a relação adimensional $\mathrm{Vp} /\left(\mathrm{f}_{1} \mathrm{~L}\right)$ é 0,039 , o que conduz a um fator de amplificação dinâmica $\xi$ de 2,519, considerando a taxa de amortecimento crítico já adotada.

\subsubsection{Resposta dinâmica pelo modelo discreto da NBR 6123/88}

A resposta dinâmica pelo modelo dinâmico discreto linear foi calculada com base na frequência de $0,429870 \mathrm{~Hz}, \mathrm{Vp} /\left(\mathrm{f}_{1} \mathrm{~L}\right)=0,038$, $\xi=2,590$; e demais parâmetros para a ação do vento como citados anteriormente. É válido observar que a frequência do primeiro modo de vibração, obtida pelo método proposto, conforme descrito no item 7.4.3, foi de 0,569799 Hz e, pelo Método dos Elementos Finitos, foi de $0,483026 \mathrm{~Hz}$, apresentando uma diferença de 16,42\%, decorrente do ligeiro afastamento das formas de vibração resultantes de cada método.

O cálculo da resposta dinâmica com a inclusão da não-linearidade geométrica foi feito levando-se em conta as contribuições até o $5^{\circ}$ modo de vibração. Para o primeiro modo a relação adimensional Vp/ $\left(f_{1} L\right)$ e o coeficiente de amplificação dinâmica são os mesmo do Mode- 
lo Simplificado Não-linear, presentes no item anterior. Para os modos de 2 a 5 , a frequência, a relação adimensional e o coeficiente de amplificação dinâmica são, respectivamente: 2,32969 Hz, 0,008 e 1,895; $5,687144 \mathrm{~Hz}, 0,003$ e 1,613; 10,06893 Hz, 0,002 e 1,588; 15,817035 Hz, 0,001 e 1,463 .

\subsubsection{Análise dos resultados}

Inicia-se a análise dos resultados avaliando a formulação desenvolvida no método proposto por meio da intensidade do esforço normal. O modelo elaborado no programa de Elementos Finitos serve como referência. Os esforços normais na estrutura por ambos os métodos estão na Tabela 7.3.

Tabela 7.3 - Estrutura 1: esforço normal.

\begin{tabular}{|c|c|c|c|c|}
\hline $\mathbf{L}$ & Proposto & MEF & \multicolumn{2}{|c|}{ Diferença } \\
\hline $\mathbf{( m )}$ & $\mathbf{( k N )}$ & $\mathbf{( k N )}$ & Absoluta & $\mathbf{( \% )}$ \\
\hline 48,00 & 3,355520 & 3,355520 & 0,0000 & 0,000000 \\
\hline 44,00 & 6,786842 & 6,786842 & 0,0000 & $-0,000001$ \\
\hline 38,00 & 16,585633 & 16,585633 & 0,0000 & $-0,000001$ \\
\hline 32,00 & 26,964392 & 26,964392 & 0,0000 & $-0,000001$ \\
\hline 25,00 & 40,426203 & 40,426204 & 0,0000 & $-0,000002$ \\
\hline 11,00 & 70,056344 & 70,056345 & 0,0000 & $-0,000002$ \\
\hline 0,00 & 102,174047 & 102,174049 & 0,0000 & $-0,000002$ \\
\hline
\end{tabular}

A diferença entre a frequência de vibração do modo fundamental, calculada sob não-linearidade geométrica, pelo método proposto, de $0,562350 \mathrm{~Hz}$ e a obtida pelo Método dos Elementos Finitos, de $0,483026 \mathrm{~Hz}$, é de $16 \%$. 
Pela expressão adotada na NBR 6123/88, a frequência calculada para o primeiro modo de vibração foi de $0,621435 \mathrm{~Hz}, 35,13 \%$ acima da frequência calculada pela solução proposta nesta Tese.

Verificou-se um ligeiro afastamento na forma modal dada pela função trigonométrica, assumida como função de forma do método proposto, e a obtida pelo modelo não-linear pelo MEF, como será discutido ao final desta seção.

A frequência calculada pela expressão (4.19) foi de $0,403193 \mathrm{~Hz}$, o que representa uma diferença de 28,30 \% em relação ao valor exato do método.

Os resultados críticos da ação do vento na estrutura são encontrados na comparação entre a análise estática e a análise pelo modelo dinâmico simplificado linear, onde o momento fletor da análise simplificada linear alcança o valor de 1,49 vezes o valor do momento fletor da análise estática.

Embora o coeficiente de amplificação dinâmica usado no modelo simplificado não-linear seja $4,6 \%$ superior ao do modelo simplificado linear, o seu resultado, na comparação como modelo estático, situou-se abaixo do modelo simplificado linear, com uma relação entre os momentos de 1,46, o que revela a influência que a forma modal assumida no modelo simplificado linear exerce nos resultados da resposta dinâmica da estrutura.

Computando-se as contribuições até o $5^{\circ}$ modo de vibração do modelo dinâmico discreto não-linear, a relação entre o momento fletor máximo na estrutura e o resultado obtido pela análise estática é de 1,33 vezes (33,34\%), apresentando uma diferença de 399,73 kNm.

Avaliando-se os resultados obtidos nas análises discretas não-lineares verifica-se uma pequena influência da contribuição dos modos de vibração acima do fundamental, resultando em uma diferença de $0,44 \%$, quando computadas exclusivamente as contribuições do $1^{\circ}$ modo com a superposição das contribuições do $1^{\circ}$ ao $5^{\circ}$ modo, pelo critério da raiz quadrada da soma dos quadrados. As flutuações resultantes do primeiro modo de vibração são as que aportam maior contribuição para a resposta dinâmica da estrutura, 64\% da resposta total, mesmo quando computadas as contribuições até o $5^{\circ}$ modo, conforme disposto na Tabela 7.4. 
Já as resposta dinâmicas obtida pelos modelos dinâmicos discretos, linear e não-linear, levando-se em conta apenas o $1^{\circ}$ modo, guardam entre si uma reduzida diferença de $0,52 \%$. A diferença entre a frequência fundamental do modelo linear e a do modelo não-linear, de $2 \%$, elevou o coeficiente de amplificação dinâmica em 0,37\%. Com isso, o momento na estrutura foi acrescido de 8,09 kNm.

Tabela 7.4 - Estrutura 1: momentos fletores da análise discreta não-linear.

\begin{tabular}{|c|c|c|c|c|c|c|}
\hline \multirow{2}{*}{ z } & \multirow{2}{*}{$\begin{array}{l}\text { Vento } \\
\text { Médio }\end{array}$} & \multicolumn{5}{|c|}{ Flutuações } \\
\hline & & Modo1 & Modo 2 & Modo 3 & Modo 4 & Modo 5 \\
\hline (m) & (kNm) & (kNm) & (kNm) & (kNm) & (kNm) & (kNm) \\
\hline 48 & 0,00 & 0,00 & 0,00 & 0,00 & 0,00 & 0,00 \\
\hline 46 & 2,77 & 8,24 & 3,63 & 2,14 & 1,02 & 0,56 \\
\hline 44 & 6,89 & 19,59 & 8,30 & 4,58 & 1,96 & 0,91 \\
\hline 42 & 12,41 & 35,11 & 13,94 & 6,87 & 2,41 & 0,72 \\
\hline 40 & 19,69 & 55,64 & 20,20 & 8,50 & 2,15 & 0,05 \\
\hline 38 & 28,78 & 80,76 & 26,50 & 9,17 & 1,27 & 0,75 \\
\hline 36 & 39,66 & 110,14 & 32,31 & 8,71 & 0,01 & 1,36 \\
\hline 34 & 52,35 & 143,46 & 37,15 & 7,10 & 1,39 & 1,50 \\
\hline 32 & 66,84 & 180,29 & 40,67 & 4,52 & 2,56 & 1,12 \\
\hline 31 & 74,80 & 199,91 & 41,82 & 2,98 & 2,97 & 0,77 \\
\hline 30 & 83,33 & 220,34 & 42,50 & 1,30 & 3,23 & 0,34 \\
\hline 29 & 92,43 & 241,52 & 42,70 & 0,47 & 3,34 & 0,14 \\
\hline
\end{tabular}




\begin{tabular}{|c|c|c|c|c|c|c|}
\hline \multirow{2}{*}{ z } & \multirow{2}{*}{$\begin{array}{l}\text { Vento } \\
\text { Médio }\end{array}$} & \multicolumn{5}{|c|}{ Flutuações } \\
\hline & & Modo1 & Modo 2 & Modo 3 & Modo 4 & Modo 5 \\
\hline$(\mathrm{m})$ & (kNm) & (kNm) & (kNm) & (kNm) & (kNm) & (kNm) \\
\hline 28 & 102,10 & 263,39 & 42,40 & 2,26 & 3,27 & 0,62 \\
\hline 27 & 112,33 & 285,92 & 41,59 & 4,02 & 3,04 & 1,06 \\
\hline 26 & 123,10 & 309,04 & 40,28 & 5,72 & 2,66 & 1,44 \\
\hline 25 & 134,42 & 332,72 & 38,47 & 7,29 & 2,15 & 1,73 \\
\hline 24 & 146,28 & 356,92 & 36,14 & 8,68 & 1,52 & 1,89 \\
\hline 23 & 158,70 & 381,63 & 33,29 & 9,85 & 0,81 & 1,92 \\
\hline 22 & 171,68 & 406,79 & 29,93 & 10,76 & 0,05 & 1,81 \\
\hline 21 & 185,22 & 432,37 & 26,09 & 11,37 & 0,72 & 1,58 \\
\hline 20 & 199,30 & 458,32 & 21,79 & 11,67 & 1,46 & 1,23 \\
\hline 19 & 213,91 & 484,60 & 17,06 & 11,64 & 2,13 & 0,80 \\
\hline 18 & 229,05 & 511,16 & 11,94 & 11,27 & 2,70 & 0,31 \\
\hline 17 & 244,69 & 537,98 & 6,45 & 10,55 & 3,15 & 0,20 \\
\hline 16 & 260,84 & 565,01 & 0,63 & 9,50 & 3,44 & 0,69 \\
\hline 15 & 277,49 & 592,23 & 5,48 & 8,12 & 3,55 & 1,12 \\
\hline 14 & 294,61 & 619,60 & 11,86 & 6,43 & 3,49 & 1,47 \\
\hline 13 & 312,19 & 647,11 & 18,46 & 4,45 & 3,23 & 1,71 \\
\hline
\end{tabular}




\begin{tabular}{|c|c|c|c|c|c|c|}
\hline \multirow{2}{*}{$\mathbf{z}$} & \multirow{2}{*}{$\begin{array}{l}\text { Vento } \\
\text { Médio }\end{array}$} & \multicolumn{5}{|c|}{ Flutuações } \\
\hline & & Modo1 & Modo 2 & Modo 3 & Modo 4 & Modo 5 \\
\hline$(\mathrm{m})$ & (kNm) & (kNm) & (kNm) & (kNm) & (kNm) & (kNm) \\
\hline 12 & 330,24 & 674,71 & 25,25 & 2,22 & 2,79 & 1,82 \\
\hline 11 & 348,72 & 702,40 & 32,21 & 0,24 & 2,17 & 1,78 \\
\hline 10 & 367,63 & 730,16 & 39,30 & 2,90 & 1,39 & 1,60 \\
\hline 9 & 386,98 & 757,97 & 46,50 & 5,74 & 0,45 & 1,27 \\
\hline 8 & 406,78 & 785,83 & 53,80 & 8,73 & 0,63 & 0,80 \\
\hline 7 & 427,02 & 813,72 & 61,18 & 11,86 & 1,82 & 0,19 \\
\hline 6 & 447,70 & 841,63 & 68,62 & 15,09 & 3,13 & 0,52 \\
\hline 5 & 468,83 & 869,57 & 76,11 & 18,40 & 4,51 & 1,34 \\
\hline 4 & 490,38 & 897,52 & 83,63 & 21,78 & 5,96 & 2,23 \\
\hline 3 & 512,35 & 925,49 & 91,18 & 25,19 & 7,45 & 3,17 \\
\hline 2 & 534,63 & 953,45 & 98,74 & 28,63 & 8,96 & 4,15 \\
\hline 1 & 557,18 & 981,42 & 106,30 & 32,09 & 10,49 & 5,14 \\
\hline 0 & 579,96 & 1009,39 & 113,87 & 35,54 & 12,03 & 6,14 \\
\hline
\end{tabular}

A Tabela 7.5 apresenta o valor dos momentos fletores na estrutura devidos à ação do vento das análises descritas anteriormente, comparando-as ao modelo estático. 


\begin{tabular}{|c|c|c|c|c|c|c|c|c|c|c|}
\hline \multicolumn{2}{|c|}{ 总 } & & $\sum_{\underline{\Sigma}}^{\bar{E}}$ & $\begin{array}{l}8 \\
0 \\
0\end{array}$ & $\begin{array}{l}\stackrel{\llcorner}{\llcorner} \\
\stackrel{+}{\leftarrow}\end{array}$ & $\begin{array}{l}\infty \\
\stackrel{\infty}{\infty} \\
\stackrel{n}{n}\end{array}$ & $\begin{array}{l}\stackrel{1}{+} \\
\text { ்ָ }\end{array}$ & $\frac{\bar{N}}{\infty 0^{-}}$ & 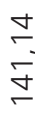 & $\begin{array}{l}\text { ᄋ } \\
\text { ᄋ́ } \\
\text { ه }\end{array}$ \\
\hline \multicolumn{2}{|c|}{ 崫 } & & $\underline{\underline{E}}$ & $\begin{array}{l}8 \\
\text { ' }\end{array}$ & $\underset{\stackrel{\sim}{\sim}}{\stackrel{一}{\leftarrow}}$ & $\begin{array}{l}\text { N } \\
\text { हे }\end{array}$ & $\begin{array}{l}\mathscr{\sigma} \\
\text { ๘ }\end{array}$ & $\begin{array}{l}0 \\
+ \\
0 \\
0\end{array}$ & $\begin{array}{l}m \\
\infty \\
\infty \\
m\end{array}$ & $\begin{array}{l}8 \\
0 \\
0 \\
\infty\end{array}$ \\
\hline \multirow{5}{*}{ 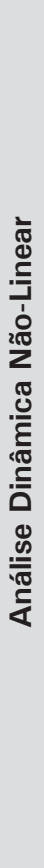 } & \multirow{5}{*}{ 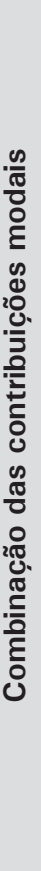 } & $\begin{array}{l}\text { L } \\
\sigma \\
\sigma\end{array}$ & $\underline{\underline{E}}_{\underline{\underline{E}}}$ & $\begin{array}{l}8 \\
\text { ○ }\end{array}$ & $\begin{array}{l}\text { o } \\
E \\
E\end{array}$ & $\begin{array}{l}\text { o } \\
\stackrel{\infty}{\sim} \\
\stackrel{0}{0}\end{array}$ & 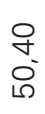 & 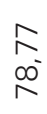 & $\begin{array}{l}\text { ㄷ } \\
\text { m) } \\
F\end{array}$ & $\begin{array}{l}\stackrel{L}{N} \\
\text { Nָ} \\
\stackrel{2}{\Omega}\end{array}$ \\
\hline & & $\begin{array}{l}+ \\
\sigma \\
\sigma\end{array}$ & $\underline{\underline{E}}$ & $\begin{array}{l}8 \\
0 \\
0\end{array}$ & $\begin{array}{l}\text { ब } \\
\text { E }\end{array}$ & $\begin{array}{l}\text { す } \\
\infty \\
\stackrel{0}{N}\end{array}$ & 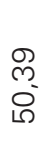 & 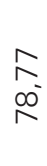 & $\begin{array}{l}\text { 三 } \\
\text { m} \\
\sigma\end{array}$ & 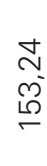 \\
\hline & & $\begin{array}{l}m \\
\sigma \\
\sigma\end{array}$ & $\underline{\underline{\varepsilon}}$ & $\begin{array}{l}8 \\
0 \\
0\end{array}$ & $\begin{array}{l}\sigma \\
\sigma\end{array}$ & $\begin{array}{l}\infty \\
\stackrel{\infty}{N} \\
\stackrel{\infty}{N}\end{array}$ & $\begin{array}{l}\text { ñ } \\
0 \\
0\end{array}$ & $\frac{m}{N}$ & $\begin{array}{l}\frac{0}{\check{m}} \\
\frac{m}{\sigma}\end{array}$ & 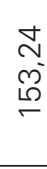 \\
\hline & & $\begin{array}{l}N \\
0 \\
\sigma\end{array}$ & $\underline{\underline{E}}$ & 응 & $\begin{array}{l}\mathscr{E} \\
E \\
E\end{array}$ & $\begin{array}{l}\text { ᄋ } \\
\text { స }\end{array}$ & $\begin{array}{l}\stackrel{P}{R} \\
\text { D্ }\end{array}$ & $\frac{m}{\infty} \frac{m}{\infty}$ & $\begin{array}{l}\hat{\theta} \\
\stackrel{1}{\sigma} \\
\sigma\end{array}$ & $\begin{array}{l}\bar{\sigma} \\
\text { No } \\
\text { م }\end{array}$ \\
\hline & & $\begin{array}{l}\text { 음 } \\
\text { 을 }\end{array}$ & $\sum_{\underline{\Sigma}}^{\bar{\varepsilon}}$ & $\begin{array}{l}8 \\
0 \\
0\end{array}$ & $\begin{array}{l}\text { ㅇ. } \\
\text { - }\end{array}$ & $\begin{array}{l}\bar{N} \\
\underset{\sim}{\sim}\end{array}$ & \begin{tabular}{l} 
寸 \\
\multirow{\sigma}{*}{}
\end{tabular} & 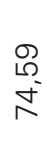 & \begin{tabular}{l}
$\stackrel{\varphi}{\sim}$ \\
$\infty$ \\
0 \\
\hdashline
\end{tabular} & 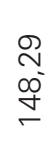 \\
\hline \multicolumn{2}{|c|}{$\overrightarrow{\mathrm{Q}}$} & & $\sum_{\underline{\Sigma}}^{\bar{E}}$ & $\begin{array}{l}8 \\
0 \\
0\end{array}$ & $\begin{array}{l}\mathscr{Q} \\
\infty \\
\stackrel{0}{ }\end{array}$ & $\frac{m}{\stackrel{m}{\sigma}}$ & $\begin{array}{l}\text { D } \\
0^{-} \\
+\end{array}$ & $\begin{array}{l}\stackrel{\mathrm{m}}{\mathrm{m}} \\
\stackrel{\mathrm{N}}{\mathrm{N}}\end{array}$ & 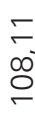 & \begin{tabular}{l}
$m$ \\
$\infty$ \\
\multirow{+}{+}{}
\end{tabular} \\
\hline 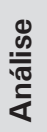 & 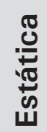 & & $\sum_{\underline{E}}^{\bar{E}}$ & $\begin{array}{l}8 \\
0 \\
0\end{array}$ & $\frac{\sigma}{\sigma}$ & $\begin{array}{l}m \\
\stackrel{a}{2} \\
\stackrel{2}{2}\end{array}$ & $\stackrel{m}{m} \underset{N}{N}$ & 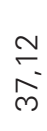 & $\begin{array}{l}\stackrel{+}{+} \\
\stackrel{+}{\circ}\end{array}$ & $\frac{m}{\sigma}$ \\
\hline & $N$ & & ह్ & $\stackrel{\infty}{+}$ & Q & ষ & F & 암 & $\stackrel{\infty}{m}$ & $\stackrel{m}{\infty}$ \\
\hline
\end{tabular}




\begin{tabular}{|c|c|c|c|c|c|c|c|c|c|c|}
\hline \multicolumn{2}{|c|}{ 岂 } & & $\begin{array}{l}\underline{\underline{E}} \\
\underline{\underline{z}}\end{array}$ & 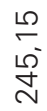 & 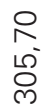 & $\stackrel{\stackrel{\llcorner}{\Omega}}{\stackrel{m}{m}}$ & 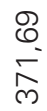 & $\begin{array}{l}\llcorner \\
\infty \\
8^{\circ} \\
\delta^{\circ}\end{array}$ & $\begin{array}{l}m \\
m \\
\stackrel{m}{\checkmark}\end{array}$ & 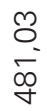 \\
\hline \multicolumn{2}{|c|}{ 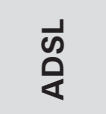 } & & $\sum_{\underline{\underline{E}}}^{\overline{\underline{E}}}$ & $\begin{array}{l}\stackrel{\mathcal{N}}{0} \\
\stackrel{+}{\sim}\end{array}$ & 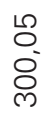 & $\stackrel{\stackrel{\mathscr{\Omega}}{\infty}}{\stackrel{m}{m}}$ & 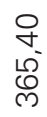 & 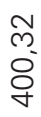 & 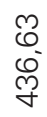 & $\begin{array}{l}\underset{\forall}{\sim} \\
\underset{\forall}{*}\end{array}$ \\
\hline \multirow{5}{*}{ 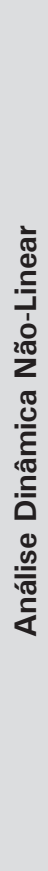 } & \multirow{5}{*}{ 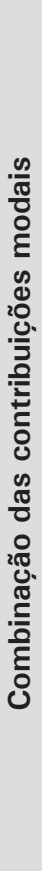 } & $\begin{array}{l}\text { I0 } \\
\text { ס } \\
\end{array}$ & $\sum_{\underline{\underline{E}}}^{\overline{\underline{E}}}$ & $\frac{m}{\infty}$ & 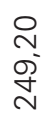 & 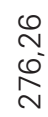 & $\begin{array}{l}\widetilde{0} \\
\text { ¿े } \\
\text { లె }\end{array}$ & $\begin{array}{l}\stackrel{L}{N} \\
\underset{\sim}{\sim} \\
\text { m }\end{array}$ & 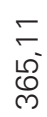 & $\frac{m}{\stackrel{n}{-}}$ \\
\hline & & $\begin{array}{l}+ \\
\text { ர } \\
-\end{array}$ & $\begin{array}{l}\overline{\underline{\xi}} \\
\text { 兰 }\end{array}$ & 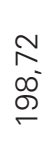 & $\begin{array}{l}\stackrel{ }{N} \\
\stackrel{\sim}{\sim}\end{array}$ & $\begin{array}{l}\mathscr{v} \\
\underset{v}{0} \\
\stackrel{N}{N}\end{array}$ & $\begin{array}{l}\mathcal{N} \\
\text { ¿ } \\
\text { లे }\end{array}$ & $\begin{array}{l}\text { N } \\
\text { N } \\
\text { m }\end{array}$ & $\begin{array}{l}\check{\sigma} \\
\stackrel{5}{0} \\
\text { हn }\end{array}$ & 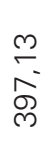 \\
\hline & & $\begin{array}{l}m \\
\text { ర } \\
-\end{array}$ & $\begin{array}{l}\overline{\underline{\varepsilon}} \\
\underline{\underline{z}}\end{array}$ & $\begin{array}{l}- \\
\text { ó } \\
\text { Oे } \\
\Gamma\end{array}$ & 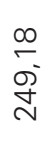 & 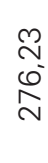 & $\begin{array}{l}\text { ¿ } \\
\text { ¿े } \\
\text { ల }\end{array}$ & $\begin{array}{l}\text { N } \\
\text { N } \\
\text { m }\end{array}$ & $\begin{array}{l}8 \\
0 \\
10 \\
0 \\
0\end{array}$ & స్ \\
\hline & & $\begin{array}{l}N \\
0 \\
-\end{array}$ & $\begin{array}{l}\overline{\underline{\varepsilon}} \\
\underline{\underline{z}}\end{array}$ & 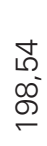 & $\begin{array}{l}\stackrel{m}{\sigma} \\
\stackrel{\sigma}{+}\end{array}$ & 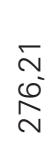 & 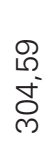 & $\begin{array}{l}\stackrel{N}{N} \\
\text { f } \\
\text { లn }\end{array}$ & $\begin{array}{l}\infty \\
0 \\
10 \\
c 0 \\
0\end{array}$ & 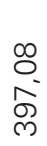 \\
\hline & & $\begin{array}{l}\overline{-} \\
\frac{0}{0} \\
\text { 일 }\end{array}$ & $\begin{array}{l}\overline{\underline{\varepsilon}} \\
\underline{\underline{z}}\end{array}$ & $\begin{array}{l}m \\
\infty \\
\text { m. } \\
\stackrel{2}{\sigma}\end{array}$ & $\begin{array}{l}\overline{0} \\
\dot{J} \\
\text { v }\end{array}$ & $\frac{\text { s. }}{\text { ล }}$ & 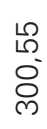 & $\begin{array}{l}\text { ○ी } \\
\text { ○' } \\
\text { ల్ }\end{array}$ & $\begin{array}{l}\text { 을 } \\
\bar{c}\end{array}$ & $\begin{array}{l}\text { व } \\
\text { j } \\
\text { गे }\end{array}$ \\
\hline \multicolumn{2}{|c|}{ 定 } & & $\sum_{\underline{\underline{\Sigma}}}^{\overline{\underline{\varepsilon}}}$ & 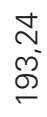 & $\begin{array}{l}\infty \\
\infty \\
\stackrel{\sim}{\sim} \\
\stackrel{\sim}{\sim}\end{array}$ & $\frac{8}{8}$ & $\begin{array}{l}0 \\
\stackrel{0}{\circ} \\
\stackrel{2}{N}\end{array}$ & $\begin{array}{l}\text { n } \\
\text { న } \\
\text { N }\end{array}$ & $\begin{array}{l}10 \\
0 \\
0 \\
0 \\
0\end{array}$ & $\begin{array}{l}\text { م } \\
\text { Nं } \\
\text { ळ }\end{array}$ \\
\hline 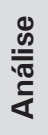 & & & $\begin{array}{l}\overline{\underline{\varepsilon}} \\
\underline{\underline{z}}\end{array}$ & $\begin{array}{l}\stackrel{L}{+} \\
\text { চ́ }\end{array}$ & $\begin{array}{l}\text { N } \\
\text { N} \\
\sim\end{array}$ & 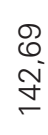 & $\begin{array}{l}\bar{N} \\
\stackrel{\sigma}{\sigma} \\
\sim\end{array}$ & $\begin{array}{l}\infty \\
\infty \\
0^{\circ} \\
\end{array}$ & 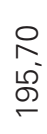 & $\begin{array}{l}0 \\
6 \\
10^{-} \\
\bar{\sim}\end{array}$ \\
\hline & $N$ & & $\overline{\mathrm{E}}$ & ले & లె & $\bar{m}$ & లి & $\stackrel{\curvearrowright}{\sim}$ & $\stackrel{\infty}{N}$ & $\lesssim$ \\
\hline
\end{tabular}




\begin{tabular}{|c|c|c|c|c|c|c|c|c|c|c|}
\hline \multicolumn{2}{|c|}{$\begin{array}{l}\underset{\infty}{2} \\
\text { 定 }\end{array}$} & & $\underset{\text { 忘 }}{\overline{\underline{E}}}$ & $\begin{array}{l}\hat{\infty} \\
\sigma^{-} \\
\bar{\sigma}\end{array}$ & $\begin{array}{l}0 \\
\circ \\
0 \\
0 \\
1\end{array}$ & $\begin{array}{l}\text { đै } \\
\text { ठ } \\
0\end{array}$ & 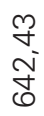 & $\begin{array}{l}- \\
\overline{10} \\
00 \\
0\end{array}$ & $\begin{array}{l}\text { o } \\
0 \\
\infty \\
\text { N } \\
\text { N }\end{array}$ & $\stackrel{\infty}{\stackrel{\infty}{N}} \underset{N}{N}$ \\
\hline \multicolumn{2}{|c|}{ 峁 } & & $\underset{\underline{\underline{E}}}{\overline{\underline{\varepsilon}}}$ & $\begin{array}{l}\hat{0} \\
\text { m. } \\
\bar{n}\end{array}$ & 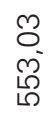 & $\begin{array}{l}\llcorner \\
\stackrel{0}{ } \\
\stackrel{5}{\circ}\end{array}$ & $\begin{array}{l}\frac{m}{6} \\
\overline{0} \\
\tilde{c} \\
0\end{array}$ & 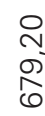 & $\begin{array}{l}\text { E } \\
\stackrel{\text { N}}{N}\end{array}$ & $\begin{array}{l}\hat{\sigma} \\
\hat{0} \\
\end{array}$ \\
\hline \multirow{5}{*}{ 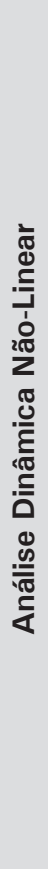 } & \multirow{5}{*}{ 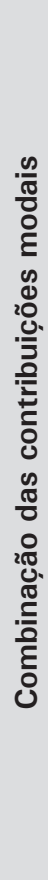 } & $\begin{array}{l}10 \\
\pi \\
-\end{array}$ & 衣 & $\begin{array}{l}\hat{N} \\
\text { ò } \\
\stackrel{y}{y}\end{array}$ & 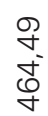 & $\begin{array}{l}\hat{\sigma} \\
\stackrel{8}{g}\end{array}$ & 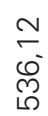 & 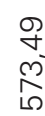 & $\begin{array}{l}\text { † } \\
\bar{\sigma} \\
\bar{\sigma}\end{array}$ & 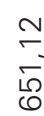 \\
\hline & & $\begin{array}{l}+ \\
\stackrel{\sigma}{\sigma}\end{array}$ & $\underset{\underline{\underline{\underline{\varepsilon}}}}{\overline{\underline{\underline{\varepsilon}}}}$ & $\begin{array}{l}\hat{N} \\
\text { Oे } \\
\stackrel{y}{+}\end{array}$ & 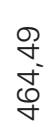 & 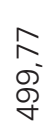 & $\begin{array}{l}\bar{E} \\
\overline{0} \\
\text { Dె }\end{array}$ & 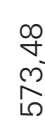 & 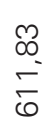 & $\frac{7}{7}$ \\
\hline & & $\begin{array}{l}m \\
\pi \\
-\end{array}$ & 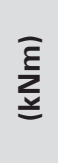 & 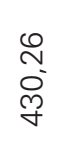 & $\begin{array}{l}\stackrel{\infty}{+} \\
\stackrel{+}{+} \\
\stackrel{+}{+}\end{array}$ & 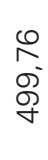 & $\begin{array}{l}\bar{E} \\
\bar{\omega} \\
\text { مె }\end{array}$ & 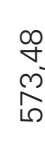 & $\begin{array}{l}\mathscr{\infty} \\
\stackrel{\infty}{\bar{\sigma}} \\
\end{array}$ & $\frac{F}{5}$ \\
\hline & & $\begin{array}{c}N \\
\stackrel{0}{-}\end{array}$ & $\begin{array}{l}\overline{\underline{\varepsilon}} \\
\text { 兰 }\end{array}$ & $\begin{array}{l}\bar{N} \\
\tilde{O} \\
\tilde{g}\end{array}$ & $\begin{array}{l}\text { 워 } \\
\text { 寸 } \\
\text { o }\end{array}$ & 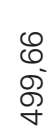 & 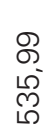 & $\begin{array}{l}\text { m } \\
\text { N} \\
\end{array}$ & $\begin{array}{l}\infty \\
\stackrel{-}{\bar{\sigma}} \\
\bar{\sigma}\end{array}$ & $\begin{array}{l}\text { o. } \\
\text { o. } \\
0\end{array}$ \\
\hline & & $\begin{array}{l}\bar{o} \\
\stackrel{\circ}{0} \\
\Sigma\end{array}$ & $\begin{array}{l}\bar{E} \\
\text { 兰 }\end{array}$ & $\underset{\stackrel{8}{\&}}{\stackrel{\&}{\Im}}$ & \begin{tabular}{l}
$\stackrel{ }{N}$ \\
$\underset{N}{O}$ \\
\multirow{\sigma}{*}{}
\end{tabular} & 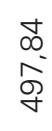 & 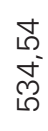 & $\begin{array}{l}\stackrel{\llcorner}{N} \\
\underset{1}{N}\end{array}$ & $\begin{array}{l}8 \\
\circ \\
0 \\
\end{array}$ & $\begin{array}{l}\text { ه̊ } \\
\circ \\
\circ \\
0 \\
0\end{array}$ \\
\hline \multicolumn{2}{|c|}{$\overrightarrow{\mathrm{Q}}$} & & $\underset{\underline{\underline{E}}}{\overline{\underline{E}}}$ & 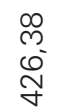 & $\begin{array}{l}\text { ' } \\
\infty \\
8 \\
8 \\
8\end{array}$ & 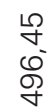 & 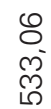 & $\begin{array}{l}\infty \\
0 \\
0 \\
0 \\
0\end{array}$ & $\begin{array}{l}\stackrel{+}{~} \\
\stackrel{8}{8} \\
\stackrel{8}{0}\end{array}$ & 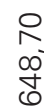 \\
\hline 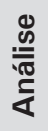 & 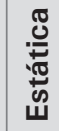 & & $\begin{array}{l}\sum_{\underline{\underline{\xi}}} \\
\end{array}$ & $\begin{array}{l}\stackrel{2}{N} \\
0 \\
\stackrel{0}{N}\end{array}$ & $\begin{array}{l}\mathscr{Q} \\
\stackrel{\circ}{ } \\
\infty \\
\stackrel{\circ}{N}\end{array}$ & $\begin{array}{l}\text { N } \\
\text { N } \\
\text { o } \\
\text { N }\end{array}$ & $\begin{array}{l}\tilde{D} \\
\infty \\
0 \\
0 \\
0\end{array}$ & $\begin{array}{l}\text { W } \\
\text { N } \\
\text { లె }\end{array}$ & $\begin{array}{l}\text { 우 } \\
\text { ò } \\
\text { लै }\end{array}$ & $\begin{array}{l}\underset{J}{*} \\
\text { o } \\
\text { m }\end{array}$ \\
\hline & $N$ & & $\underline{\xi}$ & $\stackrel{\bullet}{N}$ & 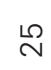 & $\stackrel{\Delta}{\sim}$ & $\stackrel{n}{N}$ & $\approx$ & $\bar{N}$ & 尺 \\
\hline
\end{tabular}




\begin{tabular}{|c|c|c|c|c|c|c|c|c|c|c|}
\hline \multicolumn{2}{|c|}{$\begin{array}{l}\text { 之 } \\
\text { 号 }\end{array}$} & & $\begin{array}{l}\overline{\underline{\xi}} \\
\underline{\underline{z}}\end{array}$ & $\begin{array}{l}\bar{\emptyset} \\
\frac{\bar{\infty}}{\bar{\infty}}\end{array}$ & $\begin{array}{l}\overline{0} \\
\text { 刃' } \\
\infty\end{array}$ & 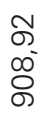 & $\begin{array}{l}\stackrel{\bullet}{N} \\
\stackrel{\sim}{\Omega} \\
\stackrel{\sim}{\sigma}\end{array}$ & $\begin{array}{l}8 \\
\text { ㅁ } \\
\text { ㅇ }\end{array}$ & 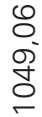 & $\begin{array}{l}\text { 워 } \\
\text { ó } \\
\text { ᄋ }\end{array}$ \\
\hline \multicolumn{2}{|c|}{ 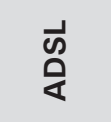 } & & $\begin{array}{l}\overline{\underline{\xi}} \\
\underline{\underline{\underline{z}}}\end{array}$ & $\begin{array}{l}\stackrel{+}{0} \\
\frac{\mathcal{m}^{-}}{\infty}\end{array}$ & $\begin{array}{l}\text { న } \\
\text { న } \\
\infty \\
\infty\end{array}$ & $\begin{array}{l}00 \\
0 \\
0 \\
0 \\
0\end{array}$ & $\begin{array}{l}+ \\
\stackrel{+}{+}\end{array}$ & $\begin{array}{l}\circ \\
\text { 응 } \\
\text { ᄋ }\end{array}$ & $\begin{array}{l}\text { N } \\
0 \\
0 \\
0 \\
0\end{array}$ & $\begin{array}{l}\text { m } \\
\text { లె } \\
\text { D }\end{array}$ \\
\hline \multirow{5}{*}{ 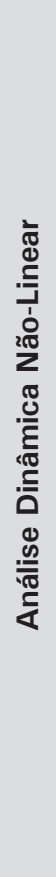 } & \multirow{5}{*}{ 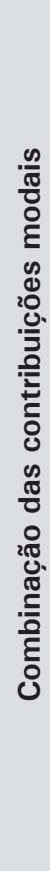 } & $\begin{array}{l}\text { L } \\
\sigma \\
\sigma\end{array}$ & $\underline{\underline{\underline{\varepsilon}}}_{\underline{\underline{z}}}$ & $\frac{\text { D }}{\text { চ }}$ & $\begin{array}{l}\text { N } \\
\text { Nं} \\
\text { N }\end{array}$ & 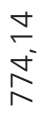 & 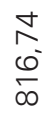 & $\begin{array}{l}8 \\
0 \\
0 \\
0 \\
\infty\end{array}$ & $\begin{array}{l}0 \\
\circ \\
\text { f } \\
\text { ठ }\end{array}$ & $\frac{N}{N}$ \\
\hline & & $\begin{array}{l}+ \\
\sigma \\
\sigma\end{array}$ & $\begin{array}{l}\overline{\underline{\xi}} \\
\underline{\underline{\underline{z}}}\end{array}$ & $\frac{\stackrel{g}{N}}{\underset{\sigma}{\sigma}}$ & $\begin{array}{l}\tilde{m} \\
\text { N } \\
N\end{array}$ & $\frac{\text { N }}{\stackrel{N}{N}}$ & $\frac{\pi}{0_{0}^{+}}$ & $\begin{array}{l}0 \\
0 \\
0 \\
0 \\
\infty\end{array}$ & $\begin{array}{l}0 \\
\circ \\
\text { ঠे } \\
\text { ه }\end{array}$ & \begin{tabular}{l}
$N$ \\
$N$ \\
$\infty$ \\
\multirow{\infty}{\infty}{}
\end{tabular} \\
\hline & & $\begin{array}{l}m \\
\sigma \\
\sigma\end{array}$ & $\begin{array}{l}\overline{\underline{\xi}} \\
\underline{\underline{z}}\end{array}$ & 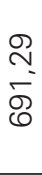 & $\begin{array}{l}\bar{m} \\
\text { N} \\
N\end{array}$ & 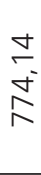 & $\begin{array}{l}m \\
\stackrel{m}{0} \\
\frac{0}{\infty}\end{array}$ & $\begin{array}{l}10 \\
0 \\
0 \\
0 \\
\infty\end{array}$ & $\begin{array}{l}\text { ᄋ } \\
\text { षे } \\
\text { ठे }\end{array}$ & $\begin{array}{l}\bar{N} \\
\infty 0^{-} \\
J \\
0\end{array}$ \\
\hline & & $\begin{array}{l}N \\
0 \\
r\end{array}$ & $\begin{array}{l}\bar{\xi} \\
\underline{\underline{z}}\end{array}$ & $\frac{12}{\check{\sigma}}$ & $\begin{array}{l}\text { న) } \\
\text { Nָ } \\
\end{array}$ & $\begin{array}{l}m \\
\text { ¿ } \\
\text { N }\end{array}$ & $\begin{array}{l}10 \\
0 \\
0 \\
0 \\
\infty\end{array}$ & $\begin{array}{l}\text { ब } \\
\text { D } \\
\infty \\
\infty\end{array}$ & $\begin{array}{l}\text { o } \\
\text { ঠे } \\
\text { ه }\end{array}$ & $\begin{array}{l}\text { ठ } \\
\infty \\
0 \\
5 \\
0\end{array}$ \\
\hline & & $\begin{array}{l}- \\
\frac{0}{0} \\
\Sigma \\
\Sigma\end{array}$ & $\begin{array}{l}\bar{\xi} \\
\underline{\underline{E}}\end{array}$ & $\begin{array}{l}\llcorner \\
\infty \\
\stackrel{\circ}{\circ} \\
\varnothing\end{array}$ & 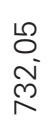 & $\begin{array}{l}\text { g } \\
\text { n' } \\
\end{array}$ & 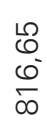 & $\begin{array}{l}\hat{\sigma} \\
\text { م } \\
\infty \\
\infty\end{array}$ & $\begin{array}{l}\bar{\sigma} \\
\text { ભ̊ } \\
\bar{\delta}\end{array}$ & \begin{tabular}{l} 
m \\
\multirow{\infty}{\infty}{} \\
o
\end{tabular} \\
\hline \multicolumn{2}{|c|}{ 虽 } & & $\begin{array}{l}\overline{\underline{\xi}} \\
\underline{\underline{z}}\end{array}$ & $\begin{array}{l}5 \\
\text { వ } \\
\infty \\
0\end{array}$ & 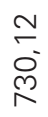 & م્ & $\begin{array}{l}+\mathbb{L} \\
\underset{\infty}{+}\end{array}$ & $\begin{array}{l}\stackrel{0}{N} \\
\hat{N} \\
\infty\end{array}$ & $\begin{array}{l}\overline{0} \\
\overline{8}\end{array}$ & 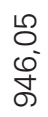 \\
\hline 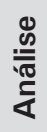 & 总 & & $\begin{array}{l}\bar{\xi} \\
\underline{\underline{z}}\end{array}$ & 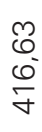 & \begin{tabular}{l}
$\stackrel{0}{\circ}$ \\
0 \\
\multirow{8}{*}{}
\end{tabular} & \begin{tabular}{l}
$\stackrel{+}{\checkmark}$ \\
$\infty$ \\
\multirow{\gamma}{\sigma}{}
\end{tabular} & $\begin{array}{l}\hat{\sigma} \\
0 \\
\overline{0}\end{array}$ & 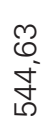 & $\begin{array}{l}\hat{m} \\
\text { o } \\
\hat{n}\end{array}$ & $\frac{\infty}{\frac{\infty}{\sigma}}$ \\
\hline & $N$ & & $\xi$ & $\stackrel{\circ}{\square}$ & $\stackrel{\infty}{\leftarrow}$ & $\approx$ & $\stackrel{\bullet}{\sigma}$ & $\stackrel{\circ 0}{\sim}$ & $\stackrel{\searrow}{\ddagger}$ & $\stackrel{m}{r}$ \\
\hline
\end{tabular}




\begin{tabular}{|c|c|c|c|c|c|c|c|c|c|c|c|}
\hline \multicolumn{2}{|c|}{ 离 } & & $\begin{array}{l}\sum_{\underline{\Sigma}} \\
\underline{\underline{z}}\end{array}$ & 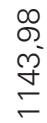 & $\begin{array}{l}\stackrel{0}{\circ} \\
\frac{5}{\sigma} \\
\check{\sigma}\end{array}$ & \begin{tabular}{l} 
․ \\
D \\
N \\
\multirow{2}{*}{}
\end{tabular} & $\begin{array}{l}\hat{N} \\
\text { No } \\
\stackrel{2}{N}\end{array}$ & 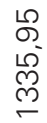 & $\begin{array}{l}\text { N } \\
\text { ঠ } \\
\infty \\
\stackrel{N}{-}\end{array}$ & 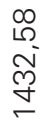 & 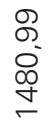 \\
\hline \multicolumn{2}{|c|}{ 虫 } & & $\begin{array}{l}\overline{\underline{E}} \\
\underline{\underline{\underline{z}}}\end{array}$ & $\begin{array}{l}\stackrel{ }{\llcorner} \\
\infty \\
\stackrel{0}{+} \\
\leftarrow\end{array}$ & 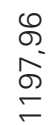 & 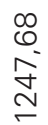 & 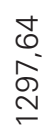 & 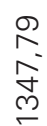 & $\begin{array}{l}\stackrel{2}{-} \\
\infty \\
\text { న }\end{array}$ & 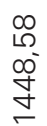 & $\begin{array}{l}\bullet \\
\stackrel{\sigma}{\sigma} \\
\stackrel{\sigma}{+}\end{array}$ \\
\hline \multirow{5}{*}{ 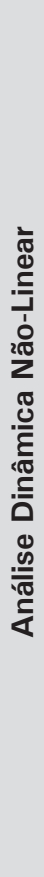 } & \multirow{5}{*}{ 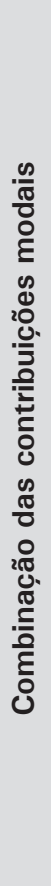 } & $\begin{array}{l}\text { م) } \\
\text { ๘ } \\
-\end{array}$ & $\begin{array}{l}\overline{\underline{E}} \\
\underline{\underline{\Sigma}}\end{array}$ & $\begin{array}{l}\text { ब } \\
\text { ஸ் } \\
\text { ब }\end{array}$ & 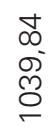 & $\begin{array}{l}\text { N } \\
\underset{0}{0} \\
\infty \\
O\end{array}$ & $\begin{array}{l}\stackrel{N}{\circ} \\
\stackrel{m}{\sigma}\end{array}$ & 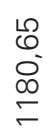 & $\begin{array}{l}\stackrel{L}{0} \\
\infty \\
\stackrel{\infty}{\sim} \\
\underset{N}{ }\end{array}$ & 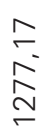 & $\begin{array}{l}\infty \\
\stackrel{\infty}{0} \\
\stackrel{2}{N} \\
\sim\end{array}$ \\
\hline & & $\begin{array}{l}\dot{\sigma} \\
\sigma \\
\sigma\end{array}$ & $\begin{array}{l}\bar{\xi} \\
\underline{\underline{E}}\end{array}$ & $\begin{array}{l}\text { ब } \\
\text { న' } \\
\text { ब }\end{array}$ & $\begin{array}{l}\text { m } \\
\infty \\
\text { లె } \\
0\end{array}$ & 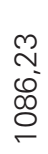 & $\begin{array}{l}\stackrel{-}{\sigma} \\
m \\
\stackrel{m}{\sigma}\end{array}$ & \begin{tabular}{l}
\multirow{0}{0}{} \\
$\circ$ \\
$\infty$ \\
-
\end{tabular} & 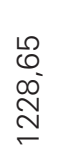 & \begin{tabular}{l}
\multirow{2}{*}{} \\
$\stackrel{N}{\sigma}$
\end{tabular} & $\begin{array}{l}\infty \\
\stackrel{-}{0} \\
\stackrel{\sim}{N} \\
\stackrel{m}{-}\end{array}$ \\
\hline & & $\begin{array}{l}m \\
\sigma \\
\sigma\end{array}$ & $\begin{array}{l}\overline{\underline{\varepsilon}} \\
\underline{\underline{z}}\end{array}$ & $\begin{array}{l}\infty \\
\text { న } \\
\text { న' }\end{array}$ & $\begin{array}{l}m \\
\infty \\
\infty \\
\infty \\
\varnothing\end{array}$ & $\begin{array}{l}\stackrel{m}{N} \\
0 \\
\infty \\
0 \\
0\end{array}$ & 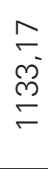 & \begin{tabular}{l}
\multirow{0}{0}{} \\
$\circ$ \\
$\infty$ \\
$\approx$
\end{tabular} & $\begin{array}{l}\stackrel{\llcorner}{0} \\
\infty \\
\stackrel{0}{\simeq} \\
\underset{\sim}{-}\end{array}$ & $\begin{array}{l}\stackrel{0}{-} \\
\stackrel{N}{N}\end{array}$ & 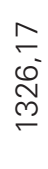 \\
\hline & & $\begin{array}{l}N \\
0 \\
\sigma\end{array}$ & $\begin{array}{l}\bar{\xi} \\
\underline{\underline{E}}\end{array}$ & $\begin{array}{l}\infty \\
\text { న } \\
\text { ల' } \\
\text { ه }\end{array}$ & $\begin{array}{l}\text { m } \\
\infty \\
\text { गे } \\
\text { ల }\end{array}$ & $\begin{array}{l}\underset{N}{N} \\
0 \\
\infty \\
O\end{array}$ & $\begin{array}{l}\stackrel{\llcorner}{-} \\
\stackrel{m}{m} \\
\rightleftharpoons\end{array}$ & $\begin{array}{l}0 \\
0 \\
\circ \\
\infty \\
\leftarrow\end{array}$ & $\begin{array}{l}\mathscr{Q} \\
\stackrel{D}{ } \\
\stackrel{\infty}{\sim} \\
\underset{\sim}{-}\end{array}$ & $\begin{array}{l}\underset{N}{N} \\
\stackrel{N}{N}\end{array}$ & $\begin{array}{l}\infty \\
\stackrel{\infty}{\infty} \\
\stackrel{1}{N} \\
\stackrel{n}{\sim} \\
\sim\end{array}$ \\
\hline & & 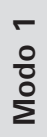 & $\begin{array}{l}\bar{\xi} \\
\underline{\underline{\Sigma}}\end{array}$ & 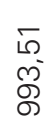 & $\begin{array}{l}\text { 음 } \\
\text { गे } \\
\text { ㅁ }\end{array}$ & 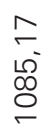 & $\begin{array}{l}m \\
\bar{m} \\
\stackrel{m}{\sigma}\end{array}$ & $\begin{array}{l}\curvearrowright \\
\infty \\
\stackrel{0}{ } \\
\approx\end{array}$ & $\begin{array}{l}\stackrel{\infty}{N} \\
\underset{\cup}{N} \\
\underset{\sim}{ }\end{array}$ & $\begin{array}{l}\stackrel{\sim}{N} \\
\underset{N}{N} \\
\stackrel{\sim}{\sim}\end{array}$ & 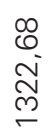 \\
\hline \multicolumn{2}{|c|}{$\overrightarrow{0}$} & & $\begin{array}{l}\underline{\underline{\xi}} \\
\underline{\underline{Z}}\end{array}$ & $\begin{array}{l}\text { m } \\
\text { চ }\end{array}$ & 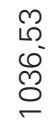 & 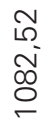 & $\begin{array}{l}\text { の } \\
\infty \\
\stackrel{0}{N} \\
\approx\end{array}$ & $\begin{array}{l}\text { ठ } \\
\stackrel{5}{\curvearrowright} \\
\approx \\
\approx\end{array}$ & $\begin{array}{l}\text { O } \\
\text { Ñ } \\
\stackrel{N}{V}\end{array}$ & 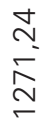 & 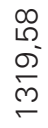 \\
\hline 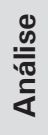 & 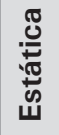 & & $\begin{array}{l}\bar{\xi} \\
\underline{\underline{\Sigma}}\end{array}$ & $\begin{array}{l}\text { ठ } \\
\text { N } \\
\text { గึ } \\
0\end{array}$ & $\begin{array}{l}\text { m } \\
\text { D' } \\
0 \\
0\end{array}$ & $\begin{array}{l}\llcorner \\
\infty \\
\infty \\
\stackrel{\infty}{N} \\
N\end{array}$ & $\begin{array}{l}\bar{\infty} \\
\infty \\
0 \\
0\end{array}$ & $\begin{array}{l}\hat{\infty} \\
\text { ') } \\
\infty\end{array}$ & $\begin{array}{l}\text { m } \\
\text { N } \\
\infty \\
\infty\end{array}$ & $\begin{array}{l}\text { ले } \\
\text { ద̊ } \\
\infty\end{array}$ & $\begin{array}{l}\text { న } \\
\text { गे } \\
\text { ñ }\end{array}$ \\
\hline & $\mathbf{N}$ & & $\overline{\mathrm{E}}$ & $\stackrel{\sim}{\leftarrow}$ & $\mp$ & 으 & $\sigma$ & $\infty$ & $N$ & 0 & ما \\
\hline
\end{tabular}




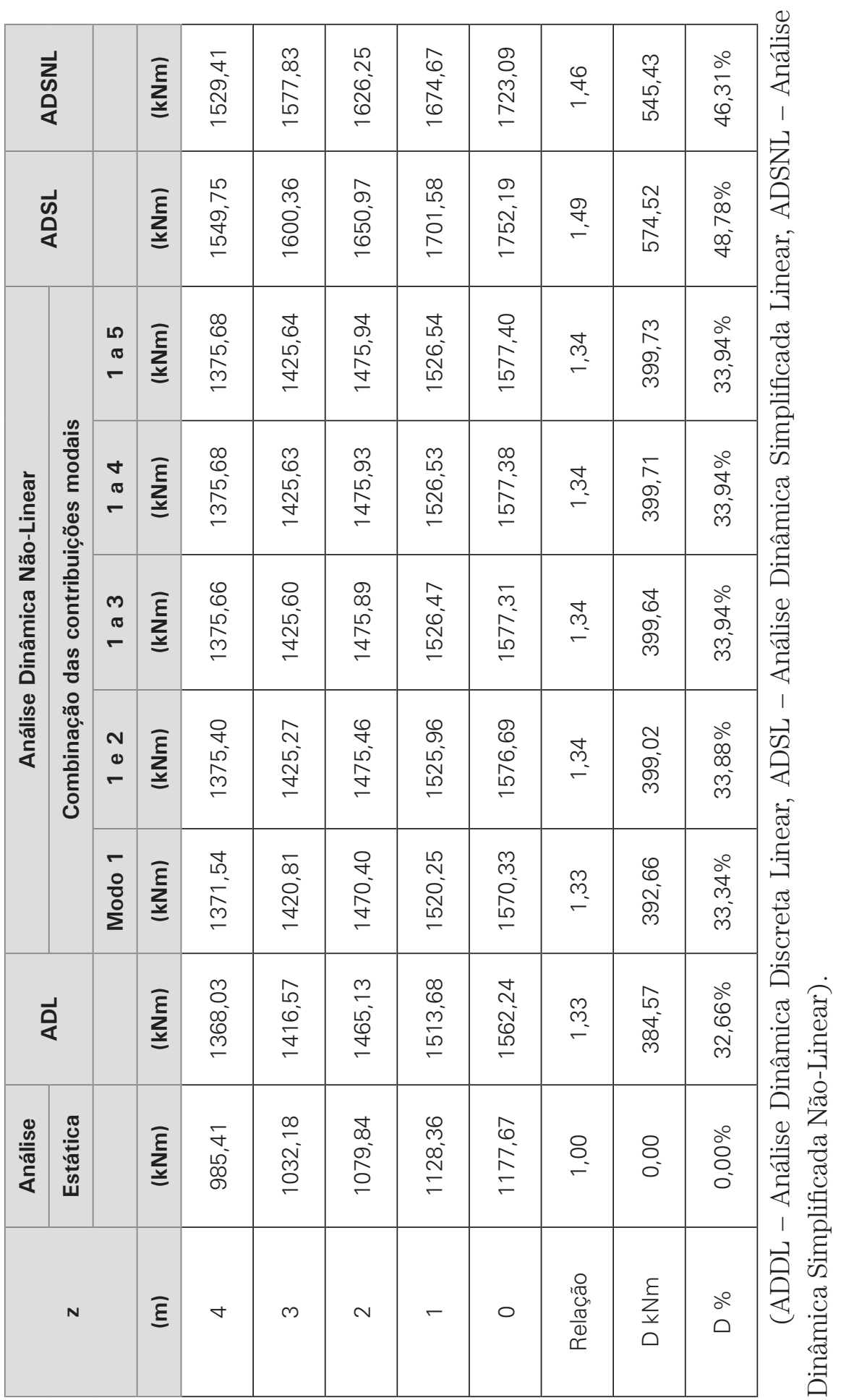


O gráfico da Figura 7.5 apresenta os resultados obtidos para a resposta da ação do vento sobre a estrutura. De baixo para cima, nesta ordem, estão: a análise estática, as análises dinâmicas discretas linear e não-linear do $1^{\circ}$ modo e modos superiores até o $5^{\circ}$ e as análises dinâmicas simplificadas.

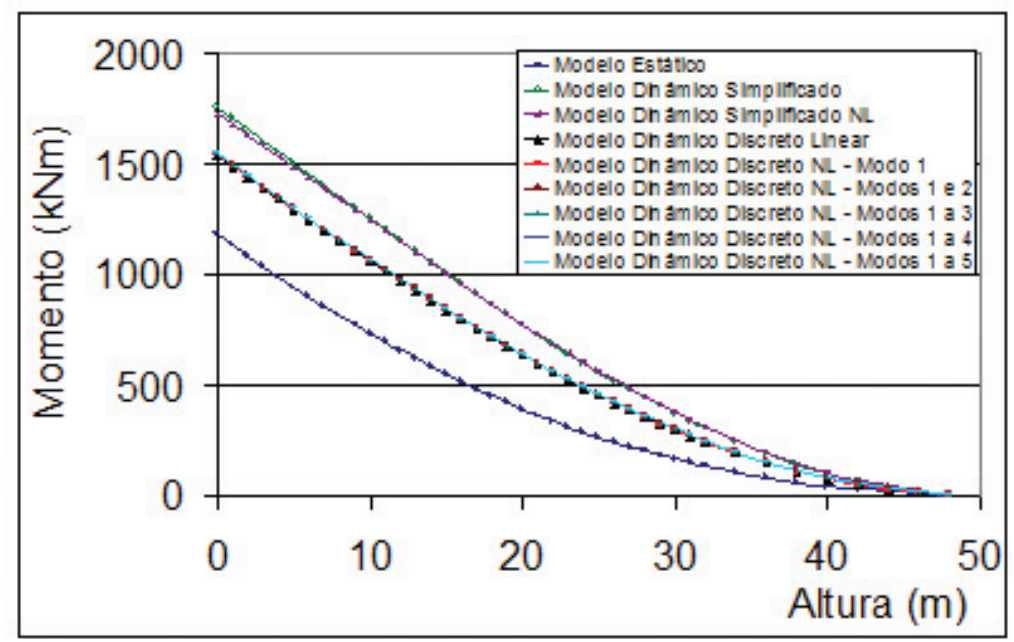

Figura 7.5 - Ação do vento na estrutura 1.

As formas modais da NBR 6123/88, a do Método dos Elementos Finitos e do método proposto constam na Figura 7.6(a).

A substituição do expoente $(\gamma)$ da forma modal sugerida pela NBR 6123/88, expressão (6.6), por 1,965, propicia uma melhor aproximação à forma modal do modelo não-linear desta estrutura, pois a curva definida com esse novo expoente oferece pontos mais próximos aos da forma modal não-linear, como pode ser observado na Figura 7.6(b).

O expoente sugerido anteriormente supera em 16\% o valor do recomendado pela NBR 6123/88. 


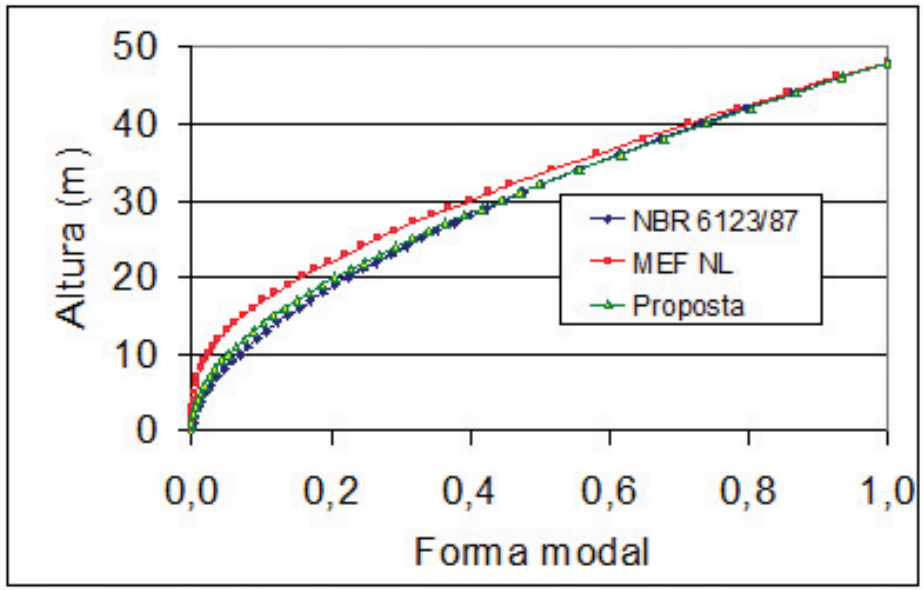

(a) expoente da forma modal: $\because=1,7$

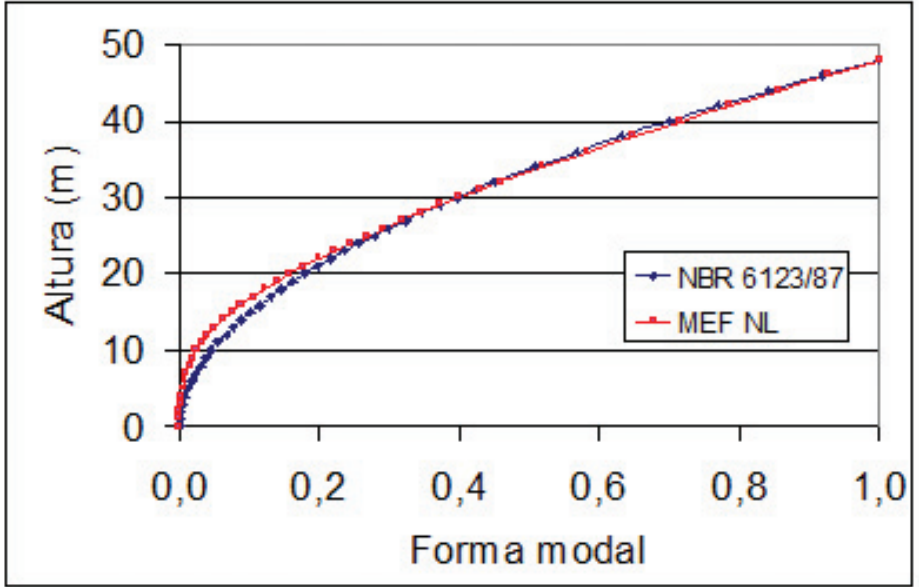

(b) expoente da forma modal: $\gamma=1,965$

Figura 7.6 - Estrutura 1 - Comparativo das formas modais: (a) usada na análise, (b) sugerida. 


\subsection{ESTRUTURA 2 - POSTE METÁLICO DE 60,80 M}

\subsubsection{Dados e geometria}

Foi analisado um poste metálico destinado também ao suporte de sistemas irradiantes de sinal de telefonia móvel celular. A estrutura possui 60,80 metros de altura e seção transversal circular vazada com diâmetro externo ( $\phi$ ext) e espessura (e) variável conforme indicação na Tabela 7.6. Na Figura 7.7 podem ser vistas fotografias da estrutura.

A Tabela 7.7 e a Figura 7.8 apresentam os parâmetros estruturais e dos dispositivos existentes, para o cálculo da ação do vento. A esbeltez da estrutura é 280. 


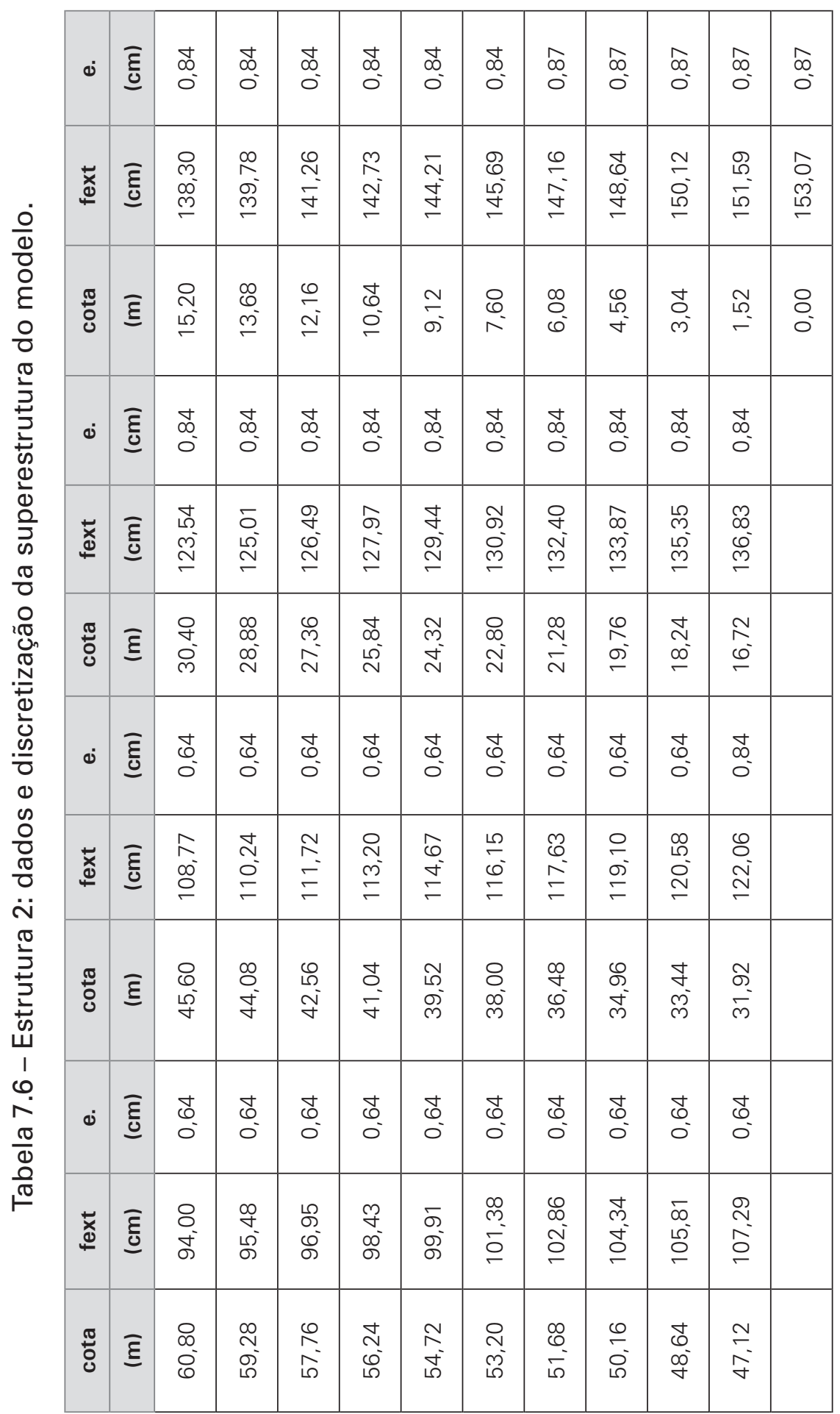


Tabela 7.7 - Estrutura 2: características dos dispositivos.

\begin{tabular}{|c|c|c|c|c|}
\hline Dispositivo & Área & Ca & Cota & $\begin{array}{c}\text { Peso específico, } \\
\text { peso distribuído } \\
\text { ou peso }\end{array}$ \\
\hline Poste & Variável & 0,6 & de 0 a $60,8 \mathrm{~m}$ & $7850 \mathrm{kN} / \mathrm{m}^{3}$ \\
\hline Escada & $0,05 \mathrm{~m}^{2} / \mathrm{m}$ & 2,0 & de 0 a $60,8 \mathrm{~m}$ & $0,15 \mathrm{kN} / \mathrm{m}$ \\
\hline Cabos & $0,15 \mathrm{~m}^{2} / \mathrm{m}$ & 1,2 & de 0 a $60,8 \mathrm{~m}$ & $0,25 \mathrm{kN} / \mathrm{m}$ \\
\hline $\begin{array}{c}\text { Antenas e } \\
\text { plataforma }\end{array}$ & $2,8 \mathrm{~m}^{2}$ & 1,0 & $60,8 \mathrm{~m}$ & $1,88 \mathrm{kN}$ \\
\hline
\end{tabular}

$(\mathrm{Ca}=$ Coeficiente de arrasto $)$
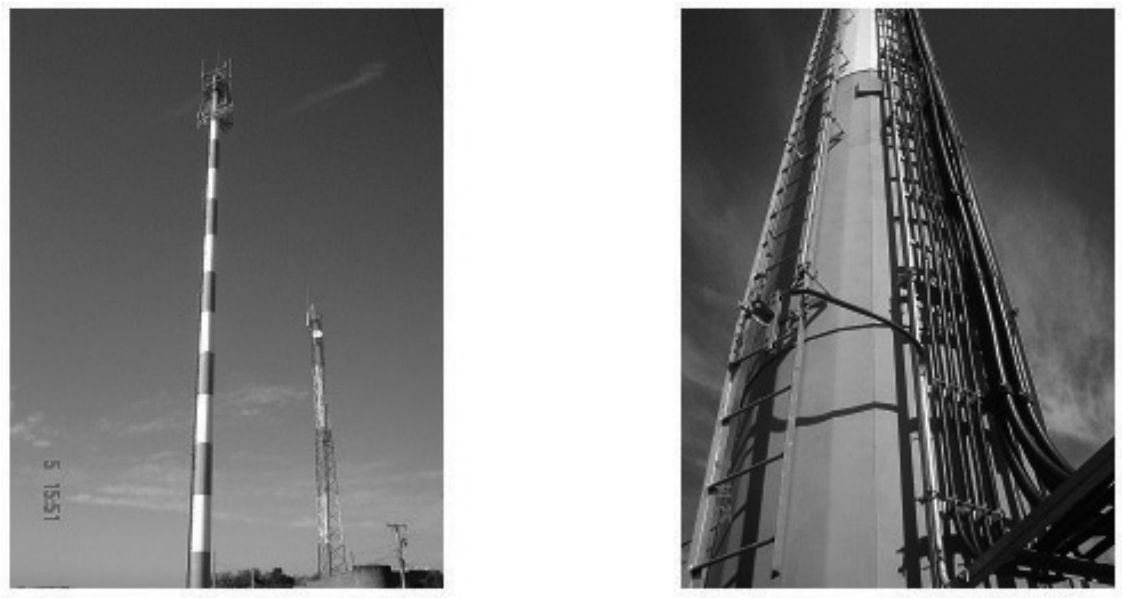

Figura 7.7 - Estrutura 2: Fotografias. 


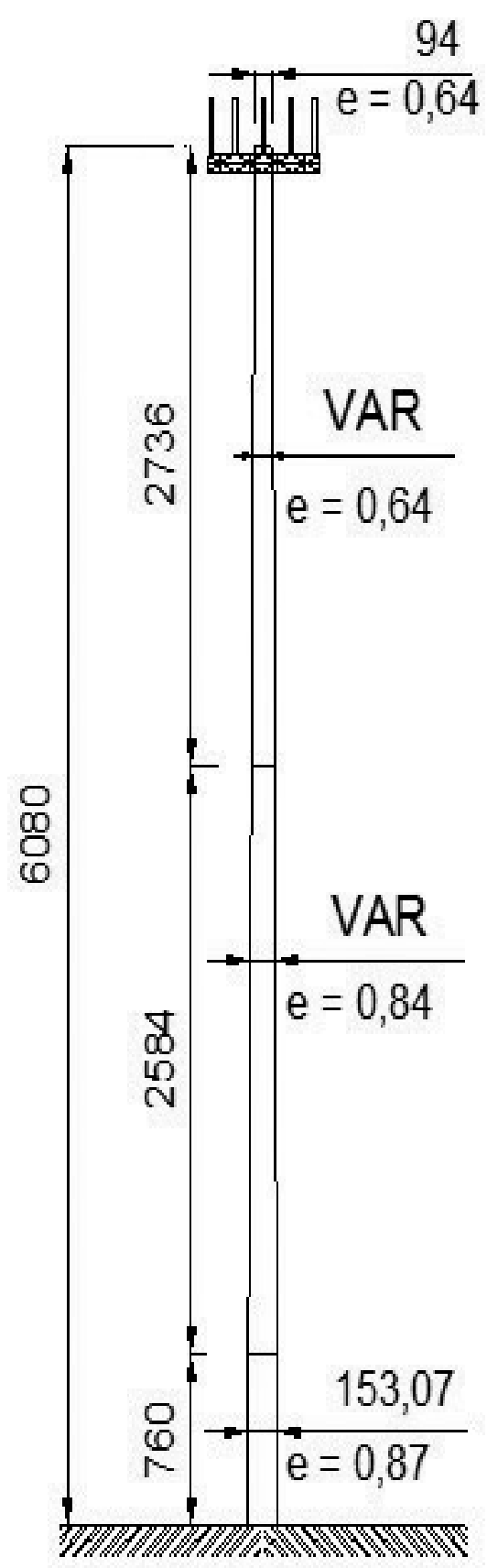

Figura 7.8 - Estrutura 2: Geometria - Poste Metálico - 60,8 m Medidas em centímetros. 


\subsubsection{Modelagem por Elementos Finitos}

A modelagem por Elementos Finitos da estrutura acompanhou os critérios já utilizados nas estruturas precedentes. A Figura 7.9 apresenta o modelo tridimensional, a vista lateral e a discretização da estrutura construídos pelo programa SAP2000.

Os modos de vibração e as frequências obtidas pelo Método dos Elementos Finitos são mostradas na Figura 7.10.

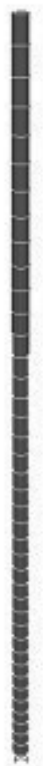

(a) $3 \mathrm{D}$

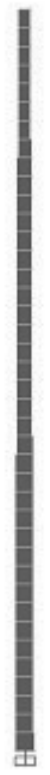

(b) Vista Lateral

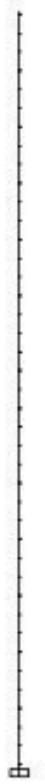

(c) Discretização

Figura 7.9 - Estrutura 2: Modelo por Elementos Finitos. 


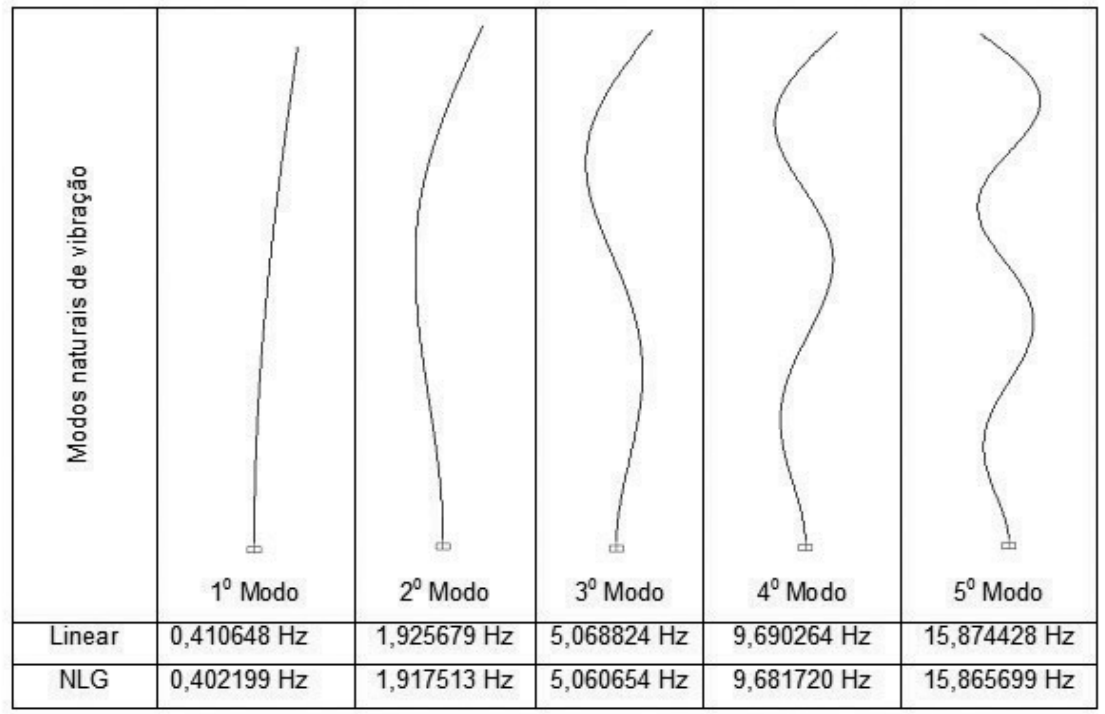

(NLG = Não-Linearidade Geométrica)

Figura 7.10 - Estrutura 2: modos naturais de vibração.

\subsubsection{Aplicação do método proposto}

\subsubsection{Definição dos dados parâmetros}

Os dados para aplicação do método foram os seguintes:

- módulo de elasticidade: $\mathrm{E}=205 \mathrm{GPa}$;

- densidade do aço: $\rho=7850 \mathrm{~kg} / \mathrm{m}^{3}$;

- massa concentrada no topo: $\mathrm{m}_{0}=451,20 \mathrm{~kg}$;

- massa distribuída por unidade de altura: $\mathrm{m}_{\mathrm{e}}=40 \mathrm{~kg} / \mathrm{m}$.

O diâmetro externo das seções varia linearmente com a altura com seguinte expressão:

$$
\mathrm{D}(\mathrm{x})=\frac{\mathrm{D}_{2}-\mathrm{D}_{1}}{\mathrm{~L}_{1}} \mathrm{x}+\mathrm{D}_{1}
$$


onde $\mathrm{D}_{2}$ é o diâmetro do topo e $\mathrm{D}_{1}$ é o diâmetro da base da estrutura. As alturas e as propriedades geométricas das seções nos trechos definidos na geometria são dadas por:

$$
\begin{aligned}
& \mathrm{L}_{1}=7,60 \mathrm{~m}, \mathrm{D}_{1}=153,07 \mathrm{~cm}, \mathrm{e}_{1}=0,87 \mathrm{~cm}, \mathrm{~d}_{1}(\mathrm{x})=\mathrm{D}(\mathrm{x})-2 \mathrm{e}_{1} \\
& \mathrm{~A}_{1}(\mathrm{x})=\frac{\pi}{4}\left(\mathrm{D}^{2}-\mathrm{d}_{1}(\mathrm{x})^{2}\right), \mathrm{I}_{1}(\mathrm{x})=\frac{\pi}{64}\left(\mathrm{D}(\mathrm{x})^{4}-\mathrm{d}_{1}(\mathrm{x})^{4}\right) \\
& \mathrm{L}_{2}=33,44 \mathrm{~m}, \mathrm{e}_{2}=0,84 \mathrm{~cm}, \mathrm{~d}_{2}(\mathrm{x})=\mathrm{D}(\mathrm{x})-2 \mathrm{e}_{2} \\
& \mathrm{~A}_{2}(\mathrm{x})=\frac{\pi}{4}\left(\mathrm{D}(\mathrm{x})^{2}-\mathrm{d}_{2}(\mathrm{x})^{2}\right), \mathrm{I}_{2}(\mathrm{x})=\frac{\pi}{64}\left(\mathrm{D}(\mathrm{x})^{4}-\mathrm{d}_{2}(\mathrm{x})^{4}\right) \\
& \mathrm{L}_{3}=60,80 \mathrm{~m}, \mathrm{D}_{2}=94 \mathrm{~cm}, \mathrm{e}_{3}=0,64 \mathrm{~cm}, \mathrm{~d}_{3}(\mathrm{x})=\mathrm{D}(\mathrm{x})-2 \mathrm{e}_{3} \\
& \mathrm{~A}_{3}(\mathrm{x})=\frac{\pi}{4}\left(\mathrm{D}(\mathrm{x})^{2}-\mathrm{d}_{3}(\mathrm{x})^{2}\right), \mathrm{I}_{3}(\mathrm{x})=\frac{\pi}{64}\left(\mathrm{D}(\mathrm{x})^{4}-\mathrm{d}_{3}(\mathrm{x})^{4}\right)
\end{aligned}
$$

\subsubsection{Cálculo da massa generalizada}

A massa generalizada foi obtida por meio das seguintes integrais:

$$
\begin{aligned}
& \mathrm{m}_{1}=\int_{0}^{\mathrm{L}_{1}} \mathrm{~m}_{\mathrm{I}}(\mathrm{x}) \phi(\mathrm{x})^{2} \mathrm{dx}, \text { com } \mathrm{m}_{\mathrm{I}}(\mathrm{x})=\mathrm{A}_{1}(\mathrm{x}) \rho+\mathrm{m}_{\mathrm{e}} \\
& \mathrm{m}_{2}=\int_{\mathrm{L}_{1}}^{\mathrm{L}_{2}} \mathrm{~m}_{\mathrm{II}}(\mathrm{x}) \phi(\mathrm{x})^{2} \mathrm{dx}, \text { com } \mathrm{m}_{\mathrm{II}}(\mathrm{x})=\mathrm{A}_{2}(\mathrm{x}) \rho+\mathrm{m}_{\mathrm{e}} \\
& \mathrm{m}_{3}=\int_{\mathrm{L}_{2}}^{\mathrm{L}} \mathrm{m}_{\mathrm{III}}(\mathrm{x}) \phi(\mathrm{x})^{2} \mathrm{dx}, \text { com } \mathrm{m}_{\mathrm{III}}(\mathrm{x})=\mathrm{A}_{3}(\mathrm{x}) \rho+\mathrm{m}_{\mathrm{e}} .
\end{aligned}
$$

A massa distribuída generalizada foi calculada por

$$
\mathrm{m}_{\mathrm{R}}=\sum_{\mathrm{i}=1}^{3} \mathrm{~m}_{\mathrm{i}}
$$


E a massa generalizada total pela expressão:

$$
\mathrm{M}=\mathrm{m}_{0}+\mathrm{m}_{\mathrm{R}}
$$

\subsubsection{Cálculo da rigidez generalizada}

\section{Rigidez geométrica generalizada}

Os esforços normais relativos aos trechos definidos na geometria, são

$$
\begin{gathered}
\mathrm{F}_{0}=\mathrm{m}_{0} \mathrm{~g}, \\
\mathrm{~F}_{3}=\int_{\mathrm{L}_{2}}^{\mathrm{L}} \mathrm{m}_{\mathrm{III}}(\mathrm{x}) \mathrm{gdx}, \\
\mathrm{F}_{2}=\int_{\mathrm{L} 1}^{\mathrm{L}_{2}} \mathrm{~m}_{\mathrm{II}}(\mathrm{x}) \mathrm{gdx}, \mathrm{e} \\
\mathrm{F}_{1}=\int_{0}^{\mathrm{L}_{1}} \mathrm{~m}_{\mathrm{I}}(\mathrm{x}) \mathrm{gdx},
\end{gathered}
$$

A força normal generalizada $\mathrm{F}$ foi obtida pela soma dessa parcelas:

$$
\mathrm{F}=\sum_{\mathrm{i}=0}^{3} \mathrm{~F}_{\mathrm{i}}
$$

As rigidezes geométricas foram calculadas pelas seguintes integrais:

$$
\begin{gathered}
\mathrm{K}_{\mathrm{g} 3}=\int_{\mathrm{L}_{2}}^{\mathrm{L}_{3}}\left[\mathrm{~F}_{0}+\mathrm{m}_{\mathrm{III}}(\mathrm{x})\left(\mathrm{L}_{3}-\mathrm{x}\right) \mathrm{g}\left(\frac{\mathrm{d}}{\mathrm{dx}} \phi(\mathrm{x})\right)^{2}\right] \\
\mathrm{K}_{\mathrm{g} 2}=\int_{\mathrm{L}_{1}}^{\mathrm{L}_{2}}\left[\mathrm{~F}_{0}+\mathrm{F}_{3}+\mathrm{m}_{\mathrm{II}}(\mathrm{x})\left(\mathrm{L}_{2}-\mathrm{x}\right) \mathrm{g}\left(\frac{\mathrm{d}}{\mathrm{dx}} \phi(\mathrm{x})\right)^{2}\right]
\end{gathered}
$$




$$
\mathrm{K}_{\mathrm{g} 1}=\int_{\mathrm{L}_{2}}^{\mathrm{L}_{3}}\left[\mathrm{~F}_{0}+\mathrm{F}_{3}+\mathrm{F}_{2}+\mathrm{m}_{\mathrm{I}}(\mathrm{x})\left(\mathrm{L}_{1}-\mathrm{x}\right) \mathrm{g}\left(\frac{\mathrm{d}}{\mathrm{dx}} \phi(\mathrm{x})\right)^{2}\right]
$$

A rigidez geométrica generalizada é:

$$
\mathrm{K}_{\mathrm{g}}=\sum_{\mathrm{i}=1}^{3} \mathrm{~K}_{\mathrm{gi}}
$$

\section{Rigidez elástica generalizada}

As parcelas da rigidez elástica para os intervalos já definidos são:

$$
\begin{aligned}
& \mathrm{K}_{01}=\int_{0}^{\mathrm{L}_{1}} \mathrm{EI}_{1}(\mathrm{x})\left(\frac{\mathrm{d}^{2}}{\mathrm{dx}^{2}} \phi(\mathrm{x})\right)^{2} \mathrm{dx}, \\
& \mathrm{K}_{02}=\int_{\mathrm{L}_{1}}^{\mathrm{L}_{2}} \mathrm{E}_{1} \mathrm{I}_{2}(\mathrm{x})\left(\frac{\mathrm{d}^{2}}{\mathrm{dx}^{2}} \phi(\mathrm{x})\right)^{2} \mathrm{dx} \mathrm{e} \\
& \mathrm{K}_{03}=\int_{\mathrm{L}_{2}}^{\mathrm{L}} \mathrm{EI}_{3}(\mathrm{x})\left(\frac{\mathrm{d}^{2}}{\mathrm{dx}^{2}} \phi(\mathrm{x})\right)^{2} \mathrm{dx} .
\end{aligned}
$$

$\mathrm{E}$ a rigidez à flexão generalizada $\mathrm{K}_{0}$ é, portanto:

$$
\mathrm{K}_{0}=\sum_{\mathrm{i}=1}^{3} \mathrm{~K}_{0 \mathrm{i}}
$$

\subsubsection{Cálculo da frequência}

As frequências do primeiro modo de vibração da estrutura pelo método proposto são: modelo linear $=0,411504 \mathrm{~Hz}$, modelo não-linear $=0,403130 \mathrm{~Hz}$. 


\subsubsection{Ação do vento}

\subsubsection{Forças estáticas devidas ao vento}

Os parâmetros empregados na determinação das forças estáticas devidas a ação do vento foram: fator topográfico $\mathrm{S}_{1}=1,2$; fator de rugosidade do terreno $\mathrm{S}_{2}$ correspondente à categoria III, classe C, com os parâmetros $\mathrm{p}=0,115, \mathrm{~b}=0,930$ e $\mathrm{F}_{\mathrm{r}}=0,950$; fator estatístico $\mathrm{S}_{3}=1,1$; velocidade básica do vento $\mathrm{V}_{0}=40 \mathrm{~m} / \mathrm{s}$.

\subsubsection{Resposta dinâmica pelo modelo simplificado da NBR 6123/88}

Os parâmetros usados na determinação da resposta dinâmica pelo modelo contínuo simplificado da NBR 6123/88 foram os seguintes: largura da edificação 1,235 m; altura da edificação para o cálculo da frequência $60,80 \mathrm{~m}$; categoria do terreno, velocidade básica do vento e fatores estatísticos como descrito no item anterior. A frequência fundamental foi obtida fazendo $T_{1}=0,29 \sqrt{\mathrm{h}}-0,4$ (Tabela 6.4), $\log \mathrm{f}_{1}=0,537272 \mathrm{~Hz}$. A forma modal foi obtida pela expressão (6.6) com $\gamma$ igual a 1,7. A relação adimensional $\mathrm{Vp} /\left(\mathrm{f}_{1} \mathrm{~L}\right)$, para esse caso, é 0,038. Adotando a taxa de amortecimento crítico $\zeta$ igual a 0,008 chega-se a um coeficiente de amplificação dinâmica $\xi$ igual a 2,471 .

Para desenvolvimento do modelo simplificado não-linear, tomou-se a frequência e o modo de vibração da estrutura sob não-linearidade geométrica. A frequência do modelo não-linear é 0,402115 Hz. Com isso, a relação adimensional $\mathrm{Vp} /\left(\mathrm{f}_{1} \mathrm{~L}\right)$ é de 0,050 , o que conduz a um fator de amplificação dinâmica $\xi=2,603$, considerando a taxa de amortecimento crítico já adotada. 


\subsubsection{Resposta dinâmica pelo modelo discreto da NBR 6123/88}

A resposta dinâmica pelo modelo dinâmico discreto linear foi calculada com base nos parâmetros e relações apresentadas no item anterior.

Os parâmetros usados no cálculo da resposta dinâmica pelo modelo discreto, com a inclusão da não-linearidade geométrica, foram descritos na análise simplificada não-linear, apresentada também no item anterior. Para os modos de 2 a 5 os fatores de amplificação dinâmica, dadas as frequências mostradas na Figura 7.10, são, respectivamente: 1,$963 ; 1,647 ; 1,599 ; 1,463$.

\subsubsection{Análise dos resultados}

Os esforços normais na estrutura pelo método proposto e pelo MEF estão na Tabela 7.13.

Tabela 7.8 - Estrutura 2: esforço normal.

\begin{tabular}{|c|c|c|c|c|}
\hline $\mathbf{L}$ & Proposto & MEF & \multicolumn{2}{|c|}{ Diferença } \\
\hline $\mathbf{( m )}$ & $(\mathbf{k N})$ & $\mathbf{( k N )}$ & Absoluta & $(\%)$ \\
\hline 60,80 & 4,421760 & 4,421760 & 0,0000 & 0,0000000 \\
\hline 33,44 & 60,275586 & 60,275587 & 0,0000 & $-0,0000017$ \\
\hline 7,60 & 139,796072 & 139,796074 & 0,0000 & $-0,0000018$ \\
\hline 0,00 & 166,504082 & 166,504085 & 0,0000 & $-0,0000018$ \\
\hline
\end{tabular}

A frequência do primeiro modo de vibração obtida pelo método proposto, como descrito no item 7.4.3, foi de $0,403130 \mathrm{~Hz}$ e pelo Método dos Elementos Finitos foi de 0,402115 Hz, apresentando uma diferença de $0,23 \%$. 
Encontra-se o valor de 0,307058 Hz no cálculo dessa frequência quando feita pela expressão (4.19), o que representa uma diferença de 23,83\% em relação ao cálculo exato; de 23,66\% em relação ao MEF e de 42,85\% em relação à expressão adotada da NBR 6123/88 (0,537272 Hz).

Os resultados críticos para a ação do vento na estrutura voltam a ser encontrados na comparação entre o modelo estático e os modelos dinâmicos simplificados. Com o modelo dinâmico simplificado não-linear, a relação entre o momento fletor máximo na estrutura é de 2,22 vezes, que representa uma diferença $3827,93 \mathrm{kNm}(122,41 \%)$.

Nas análises discretas não-lineares verifica-se uma pequena influência da contribuição dos modos de vibração acima do fundamental. Quando computadas as contribuições do vento médio e as flutuações do $1^{\circ}$ ao $5^{\circ}$ modo, pelo critério da raiz quadrada da soma dos quadrados, surge uma diferença de 1,56\% em relação à análise que inclui apenas a combinação do vento médio com o primeiro modo. As flutuações resultantes do primeiro modo de vibração são as que aportam maior contribuição na resposta da estrutura, retendo $67 \%$ do esforço total, quando se adiciona exclusivamente o primeiro modo de vibração.

A diferença entre a análise discreta não-linear e análise das forças estáticas, prevista no item 4 da NBR 6123/88 (modelo estático), é de 60,90\%, conforme mostrado na Tabela 7.9 .

Analisando os resultados obtidos da resposta dinâmica dos modelos discretos, linear e não-linear, observa-se uma diferença na frequência do primeiro modo de $2,10 \%$ e do fator de amplificação dinâmica de $0,38 \%$. Com isso, o momento na estrutura foi acrescido de 75,60 kNm, uma diferença de 1,55\%.

A Tabela 7.9 apresenta o valor dos momentos fletores do vento médio e das flutuações correspondentes aos modos de vibração de 1 a 5, da análise dinâmica discreta não-linear. 
Tabela 7.9 - Estrutura 2: momentos fletores da análise discreta não-linear.

\begin{tabular}{|c|c|c|c|c|c|c|}
\hline \multirow{2}{*}{$\mathbf{z}$} & \multirow{2}{*}{$\begin{array}{l}\text { Vento } \\
\text { Médio }\end{array}$} & \multicolumn{5}{|c|}{ Flutuações } \\
\hline & & Modo1 & Modo 2 & Modo 3 & Modo 4 & Modo 5 \\
\hline (m) & (kNm) & (kNm) & $(\mathrm{kNm})$ & (kNm) & (kNm) & $(k N m)$ \\
\hline 60,80 & 0,00 & 0,00 & 0,00 & 0,00 & 0,00 & 0,00 \\
\hline 59,28 & 5,98 & 12,58 & 3,39 & 0,69 & 0,65 & 0,22 \\
\hline 57,76 & 13,91 & 31,01 & 8,10 & 1,56 & 1,36 & 0,41 \\
\hline 56,24 & 23,82 & 55,14 & 13,79 & 2,46 & 1,90 & 0,45 \\
\hline 54,72 & 35,70 & 84,78 & 20,15 & 3,26 & 2,06 & 0,28 \\
\hline 53,20 & 49,55 & 119,74 & 26,85 & 3,82 & 1,68 & 0,17 \\
\hline 51,68 & 65,38 & 159,84 & 33,58 & 4,03 & 0,66 & 0,90 \\
\hline 50,16 & 83,19 & 204,89 & 40,04 & 3,80 & 1,05 & 1,86 \\
\hline 48,64 & 102,98 & 254,68 & 45,92 & 3,05 & 3,42 & 2,99 \\
\hline 47,12 & 124,74 & 309,03 & 50,96 & 1,75 & 6,38 & 4,16 \\
\hline 45,60 & 148,49 & 367,73 & 54,89 & 0,13 & 9,78 & 5,29 \\
\hline 44,08 & 174,21 & 430,58 & 57,48 & 2,59 & 13,46 & 6,26 \\
\hline 42,56 & 201,90 & 497,37 & 58,51 & 5,59 & 17,23 & 6,99 \\
\hline 41,04 & 231,56 & 567,89 & 57,81 & 9,07 & 20,87 & 7,43 \\
\hline
\end{tabular}




\begin{tabular}{|c|c|c|c|c|c|c|}
\hline \multirow{2}{*}{ z } & \multirow{2}{*}{$\begin{array}{l}\text { Vento } \\
\text { Médio }\end{array}$} & \multicolumn{5}{|c|}{ Flutuações } \\
\hline & & Modo1 & Modo 2 & Modo 3 & Modo 4 & Modo 5 \\
\hline (m) & (kNm) & (kNm) & (kNm) & (kNm) & (kNm) & (kNm) \\
\hline 39,52 & 263,18 & 641,95 & 55,21 & 12,96 & 24,19 & 7,56 \\
\hline 38,00 & 296,76 & 719,34 & 50,59 & 17,18 & 27,04 & 7,43 \\
\hline 36,48 & 332,28 & 799,85 & 43,85 & 21,61 & 29,27 & 7,07 \\
\hline 34,96 & 369,75 & 883,29 & 34,92 & 26,15 & 30,80 & 6,59 \\
\hline 33,44 & 409,14 & 969,45 & 23,76 & 30,69 & 31,61 & 6,08 \\
\hline 31,92 & 450,44 & 1058,27 & 10,27 & 35,10 & 31,68 & 5,63 \\
\hline 30,40 & 493,65 & 1149,93 & 5,92 & 39,25 & 30,98 & 5,39 \\
\hline 28,88 & 538,75 & 1244,32 & 24,84 & 42,98 & 29,64 & 5,43 \\
\hline 27,36 & 585,71 & 1341,20 & 46,42 & 46,20 & 27,83 & 5,81 \\
\hline 25,84 & 634,52 & 1440,35 & 70,56 & 48,81 & 25,75 & 6,56 \\
\hline 24,32 & 685,16 & 1541,56 & 97,15 & 50,72 & 23,62 & 7,65 \\
\hline 22,80 & 737,61 & 1644,61 & 126,03 & 51,88 & 21,68 & 9,03 \\
\hline 21,28 & 791,83 & 1749,31 & 157,06 & 52,25 & 20,13 & 10,62 \\
\hline 19,76 & 847,81 & 1855,47 & 190,07 & 51,82 & 19,17 & 12,31 \\
\hline 18,24 & 905,51 & 1962,90 & 224,88 & 50,58 & 18,96 & 13,98 \\
\hline
\end{tabular}




\begin{tabular}{|c|c|c|c|c|c|c|}
\hline \multirow{2}{*}{ z } & \multirow{2}{*}{$\begin{array}{l}\text { Vento } \\
\text { Médio }\end{array}$} & \multicolumn{5}{|c|}{ Flutuações } \\
\hline & & Modo1 & Modo 2 & Modo 3 & Modo 4 & Modo 5 \\
\hline (m) & (kNm) & (kNm) & (kNm) & (kNm) & (kNm) & (kNm) \\
\hline 16,72 & 964,89 & 2071,42 & 261,30 & 48,57 & 19,61 & 15,53 \\
\hline 15,20 & 1025,91 & 2180,88 & 299,13 & 45,83 & 21,20 & 16,83 \\
\hline 13,68 & 1088,54 & 2291,11 & 338,20 & 42,40 & 23,76 & 17,81 \\
\hline 12,16 & 1152,73 & 2401,99 & 378,30 & 38,37 & 27,27 & 18,41 \\
\hline 10,64 & 1218,42 & 2513,37 & 419,25 & 33,82 & 31,68 & 18,60 \\
\hline 9,12 & 1285,55 & 2625,15 & 460,89 & 28,83 & 36,88 & 18,39 \\
\hline 7,60 & 1354,05 & 2737,22 & 503,05 & 23,49 & 42,75 & 17,81 \\
\hline 6,08 & 1423,85 & 2849,50 & 545,58 & 17,89 & 49,15 & 16,91 \\
\hline 4,56 & 1494,86 & 2961,92 & 588,37 & 12,10 & 55,94 & 15,77 \\
\hline 3,04 & 1566,79 & 3074,42 & 631,31 & 6,20 & 62,98 & 14,47 \\
\hline 1,52 & 1639,53 & 3186,95 & 674,33 & 0,24 & 70,15 & 13,08 \\
\hline 0,00 & 1712,90 & 3299,49 & 717,36 & 5,74 & 77,36 & 11,67 \\
\hline
\end{tabular}

A Tabela 7.10 resume os valores dos momentos fletores na estrutura devidos à ação do vento, obtidos nas análises descritas anteriormente, comparando-as ao modelo estático. 


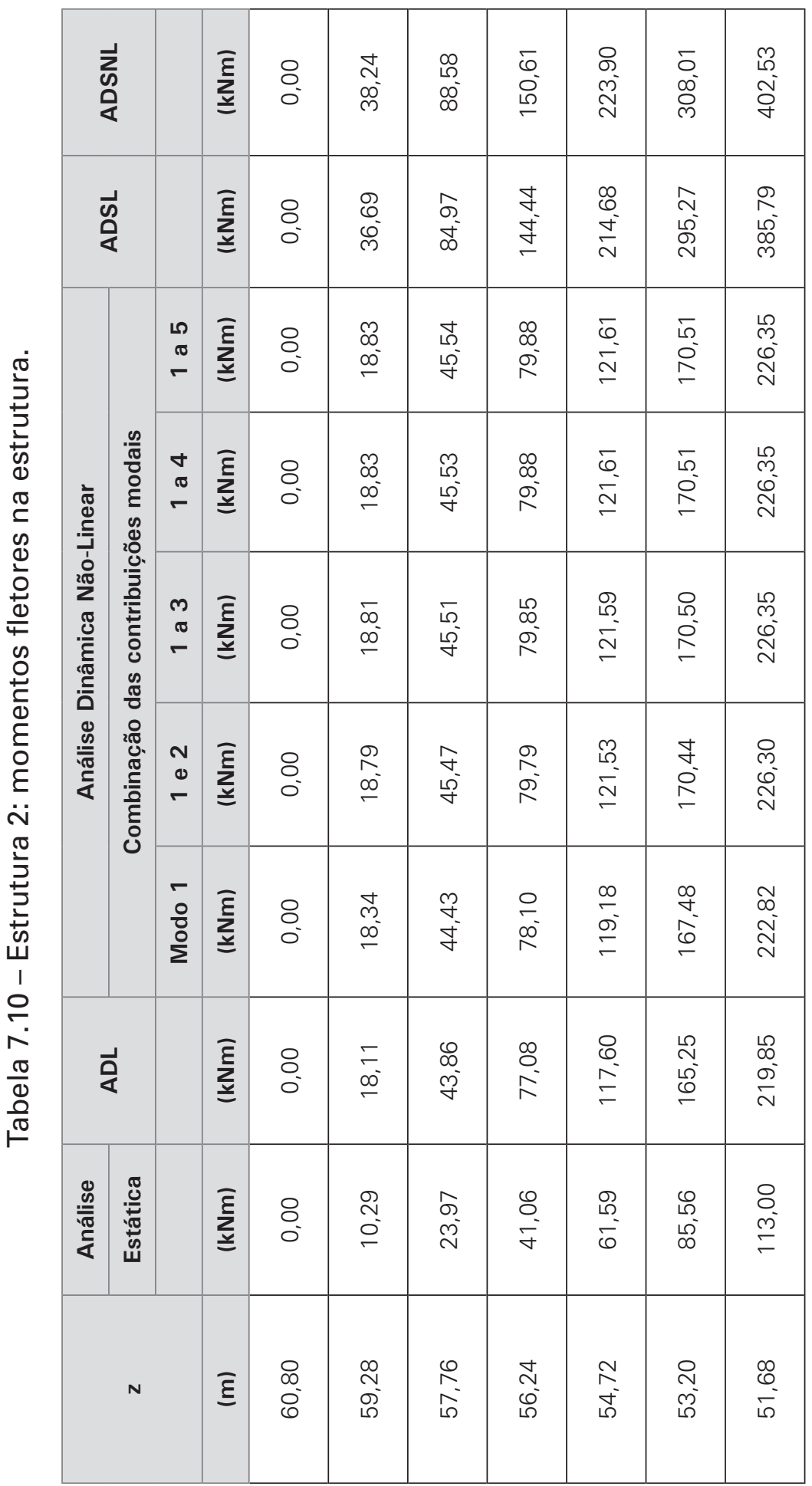




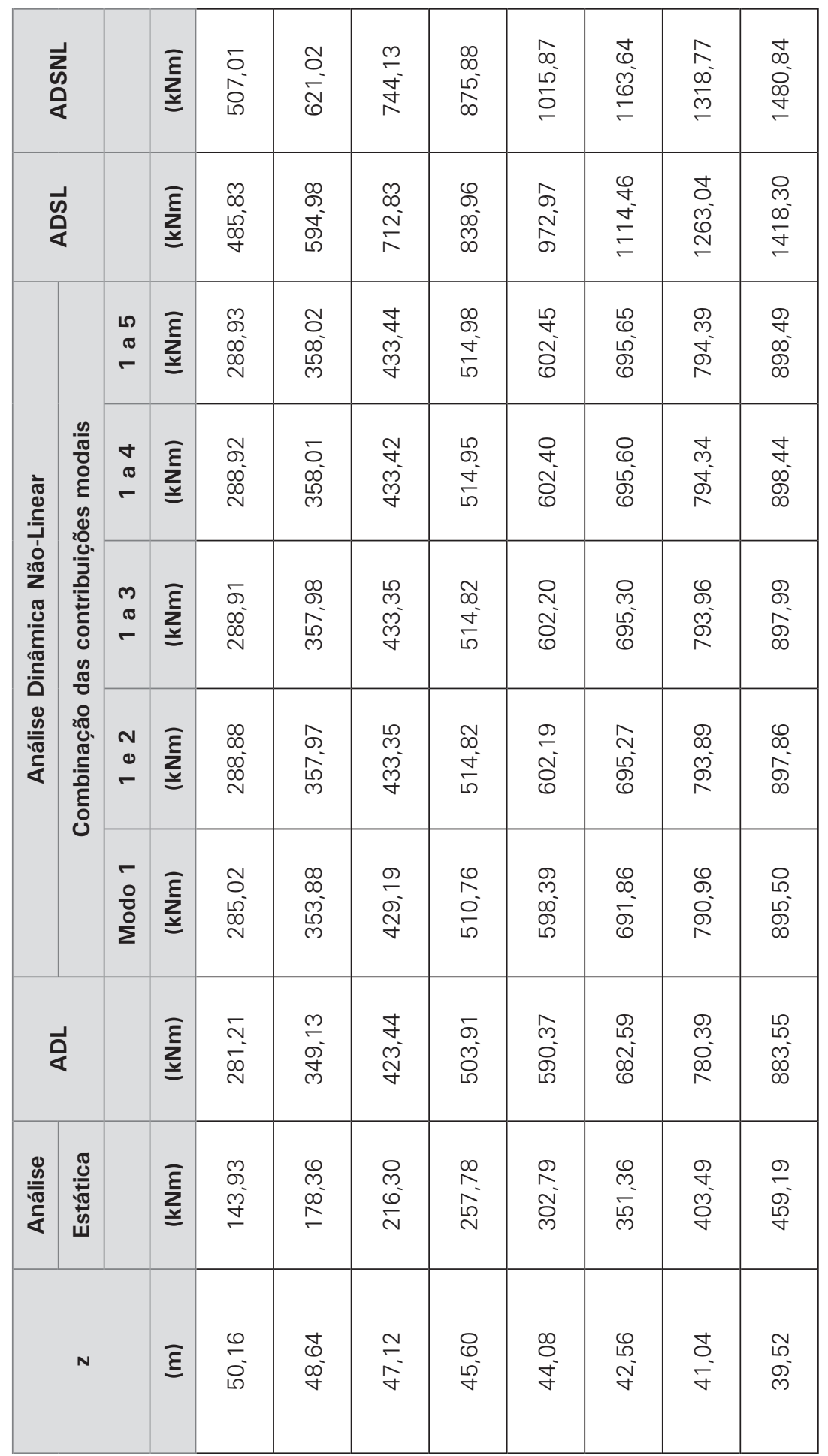




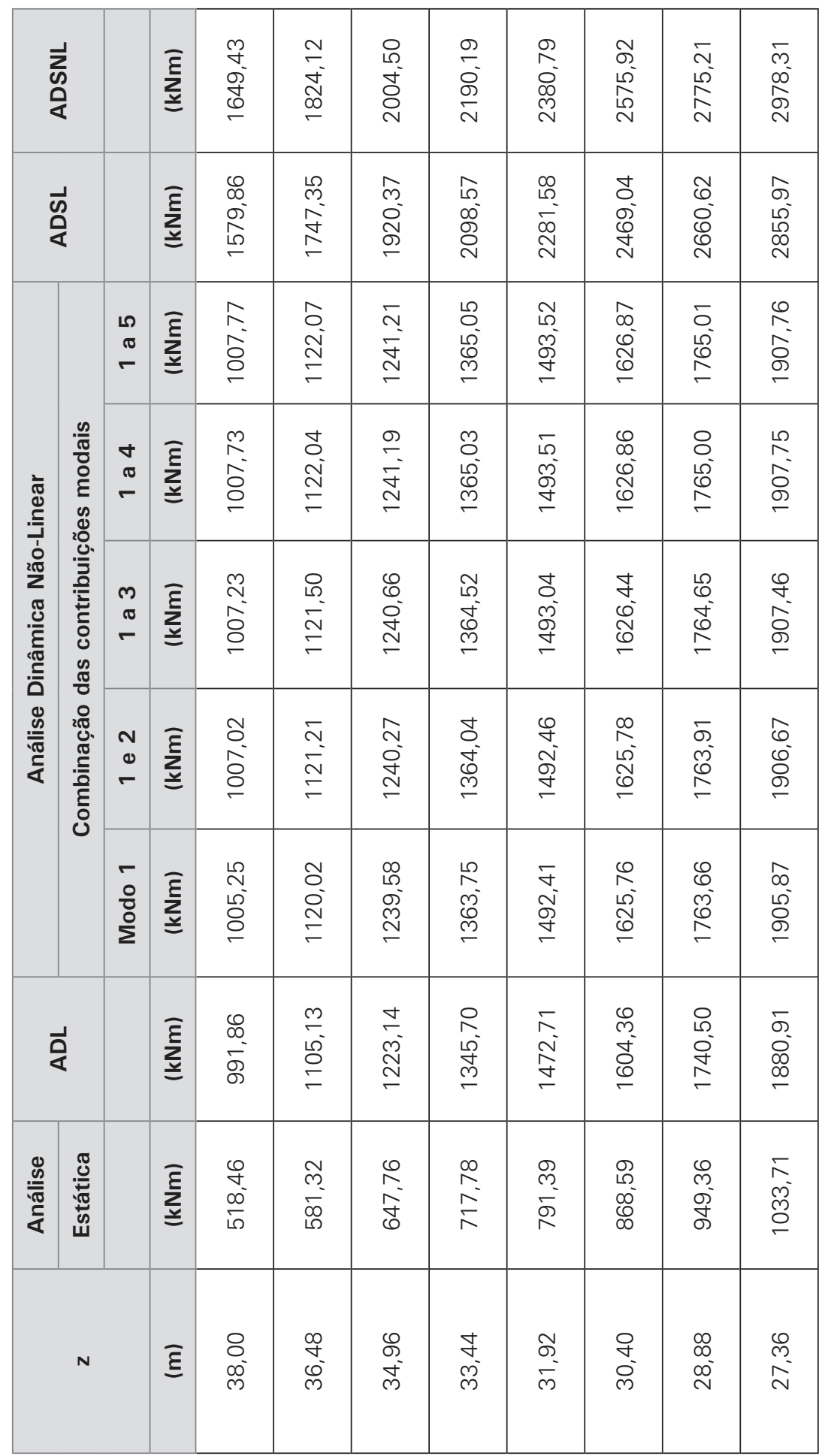




\begin{tabular}{|c|c|c|c|c|c|c|c|c|c|c|c|}
\hline \multicolumn{2}{|c|}{ 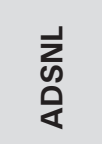 } & & $\begin{array}{l}\bar{\xi} \\
\text { E }\end{array}$ & 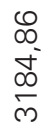 & $\begin{array}{l}\text { న } \\
\text { 尺 } \\
\text { ల్ }\end{array}$ & $\begin{array}{l}\text { ○ } \\
\text { ஸ̊ } \\
\stackrel{0}{0}\end{array}$ & 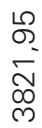 & 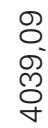 & 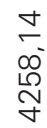 & \begin{tabular}{l}
$\mathscr{N}$ \\
$\infty$ \\
$\infty$ \\
\multirow{8}{*}{}
\end{tabular} & $\begin{array}{l}\text { ఠু } \\
8 \\
\stackrel{8}{\gamma}\end{array}$ \\
\hline \multicolumn{2}{|c|}{ 㟔 } & & $\begin{array}{l}\bar{\xi} \\
\bar{\sum}\end{array}$ & 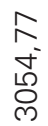 & $\begin{array}{l}- \\
0 \\
\stackrel{0}{0} \\
\stackrel{N}{N}\end{array}$ & $\begin{array}{l}\hat{f} \\
\bar{\sigma} \\
\dot{\sigma} \\
\text { ले }\end{array}$ & $\begin{array}{l}0 \\
\stackrel{0}{0} \\
0 \\
0 \\
0 \\
\text { ले }\end{array}$ & $\begin{array}{l}\stackrel{0}{m} \\
\infty \\
\infty \\
\infty \\
\infty\end{array}$ & $\begin{array}{l}\mathcal{1} \\
\infty \\
\infty \\
\infty \\
\infty \\
\dot{q}\end{array}$ & $\begin{array}{l}\text { o } \\
\text { ஸे } \\
\text { Oे }\end{array}$ & \begin{tabular}{l}
$\infty$ \\
$\stackrel{\infty}{\sigma}$ \\
\multirow{\sigma}{\sigma}{}
\end{tabular} \\
\hline \multirow{5}{*}{ 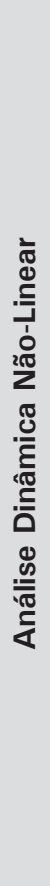 } & \multirow{5}{*}{ 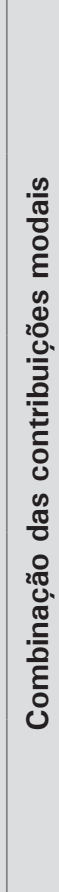 } & $\begin{array}{l}0 \\
\pi \\
\sigma\end{array}$ & $\begin{array}{l}\bar{\xi} \\
\sum_{\underline{E}}\end{array}$ & 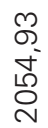 & \begin{tabular}{l}
$\bar{n}$ \\
$\stackrel{0}{0}$ \\
\multirow{N}{N}{}
\end{tabular} & 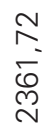 & 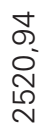 & $\begin{array}{l}\hat{\gamma} \\
\text { No } \\
0 \\
\mathscr{N}\end{array}$ & 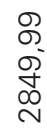 & 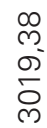 & $\frac{\nabla}{\sigma}$ \\
\hline & & $\begin{array}{l}\sigma \\
\sigma \\
\sigma\end{array}$ & $\begin{array}{l}\bar{\xi} \\
\text { E }\end{array}$ & \begin{tabular}{l}
$\bar{\sigma}$ \\
\multirow{}{*}{} \\
$\stackrel{\sim}{0}$
\end{tabular} & $\begin{array}{l}\text { D } \\
\underset{\sim}{0} \\
\stackrel{N}{N}\end{array}$ & 롱 & 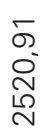 & 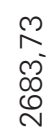 & 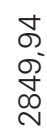 & $\begin{array}{l}m \\
m \\
\text { m } \\
\text { m }\end{array}$ & $\begin{array}{l}\infty \\
\sigma \\
\frac{\sigma}{n}\end{array}$ \\
\hline & & $\begin{array}{l}m \\
\pi \\
\sigma\end{array}$ & $\begin{array}{l}\bar{\xi} \\
\text { 方 }\end{array}$ & 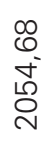 & 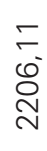 & 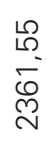 & $\begin{array}{l}\circ \\
\infty \\
\circ \\
\stackrel{\sim}{0} \\
\stackrel{1}{N}\end{array}$ & $\begin{array}{l}\text { M } \\
\text { No } \\
\infty \\
0 \\
0\end{array}$ & $\begin{array}{l}\stackrel{1}{\infty} \\
\infty \\
\stackrel{+}{+} \\
\infty \\
\sim\end{array}$ & $\begin{array}{l}\stackrel{M}{N} \\
\stackrel{\sigma}{\delta}\end{array}$ & $\frac{\hat{\sigma}}{\bar{\sigma}}$ \\
\hline & & $\begin{array}{l}N \\
0 \\
\sigma\end{array}$ & $\begin{array}{l}\bar{\xi} \\
\text { 主 }\end{array}$ & $\begin{array}{l}0 \\
\infty \\
N^{-} \\
0 \\
0\end{array}$ & $\begin{array}{l}\text { D } \\
\stackrel{2}{O} \\
\stackrel{N}{N}\end{array}$ & 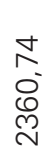 & 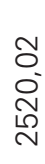 & 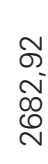 & $\begin{array}{l}\stackrel{ }{N} \\
\text { ণ্ডি } \\
\stackrel{\infty}{v}\end{array}$ & $\begin{array}{l}\hat{6} \\
\infty \\
\overline{0} \\
0\end{array}$ & $\begin{array}{l}0 \\
\frac{\sigma}{\sigma} \\
\frac{\sigma}{n}\end{array}$ \\
\hline & & $\begin{array}{l}- \\
\frac{0}{0} \\
\frac{0}{\Sigma}\end{array}$ & $\sum_{\underline{\underline{E}}}^{\bar{E}}$ & $\begin{array}{l}\stackrel{+}{\leftarrow} \\
\text { N } \\
\stackrel{\sim}{0}\end{array}$ & 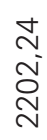 & 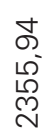 & $\begin{array}{l}N \\
\text { N } \\
\stackrel{\infty}{\sigma} \\
\sim\end{array}$ & 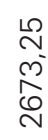 & 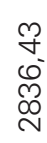 & 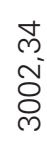 & 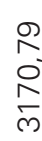 \\
\hline \multicolumn{2}{|c|}{ 完 } & & $\begin{array}{l}\bar{\xi} \\
\underline{\underline{\Sigma}}\end{array}$ & 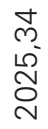 & 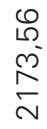 & $\begin{array}{l}\text { N } \\
\stackrel{\sim}{N} \\
\stackrel{N}{N}\end{array}$ & \begin{tabular}{l}
\multirow{\checkmark}{*}{} \\
$\stackrel{\circ}{\circ}$ \\
$\stackrel{+}{+}$
\end{tabular} & 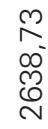 & $\begin{array}{l}\bar{\sigma} \\
\text { হ } \\
\text { ন }\end{array}$ & $\begin{array}{l}\bar{\infty} \\
\text { ల్ } \\
\text { D } \\
\text { N }\end{array}$ & $\begin{array}{l}N \\
\tilde{m} \\
\frac{n}{n}\end{array}$ \\
\hline 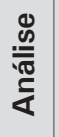 & 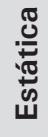 & & $\sum_{\underline{\underline{E}}}^{\bar{E}}$ & $\begin{array}{l}\bar{\sigma} \\
\check{\Sigma} \\
\check{\sigma}\end{array}$ & $\begin{array}{l}\hat{\sigma} \\
\stackrel{n}{N} \\
\stackrel{-}{-}\end{array}$ & $\begin{array}{l}9 \\
0 \\
\infty \\
0 \\
\text { m }\end{array}$ & $\begin{array}{l}0 \\
\llcorner \\
\bullet \\
\stackrel{0}{\circ} \\
\stackrel{+}{-}\end{array}$ & 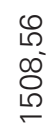 & $\begin{array}{l}\delta \\
\dot{\sigma} \\
\sigma \\
\sigma\end{array}$ & 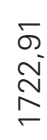 & $\begin{array}{l}\text { の } \\
\text { ம0 } \\
\infty \\
\infty\end{array}$ \\
\hline & $N$ & & छ & $\begin{array}{l}\stackrel{+}{\infty} \\
\stackrel{0}{\sim}\end{array}$ & \begin{tabular}{l} 
N \\
\multirow{N}{*}{}
\end{tabular} & \begin{tabular}{l}
$\circ$ \\
$\infty$ \\
\multirow{N}{N}{}
\end{tabular} & $\stackrel{\infty}{\stackrel{\infty}{\sim}}$ & $\begin{array}{l}\stackrel{0}{N} \\
\stackrel{\sigma}{-}\end{array}$ & $\begin{array}{l}\stackrel{+}{\sim} \\
\infty \\
\infty\end{array}$ & $\frac{N}{\sigma^{-}}$ & $\begin{array}{l}\stackrel{0}{\sim} \\
\leftarrow\end{array}$ \\
\hline
\end{tabular}




\begin{tabular}{|c|c|c|c|c|c|c|c|c|c|c|c|}
\hline \multicolumn{2}{|c|}{ 音 } & & $\bar{\sum}_{\underline{\underline{E}}}^{\bar{\varepsilon}}$ & \begin{tabular}{l}
$\stackrel{一}{\sigma}$ \\
\multirow{J}{\sigma}{} \\
$\stackrel{+}{+}$
\end{tabular} & $\begin{array}{l}\hat{m} \\
\infty \\
+ \\
\frac{0}{0}\end{array}$ & $\begin{array}{l}\text { ले } \\
\text { N } \\
\text { N }\end{array}$ & $\begin{array}{l}\text { న } \\
\infty \\
\infty \\
\infty \\
\circ \\
\circ\end{array}$ & 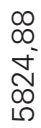 & $\begin{array}{l}\bar{\sigma} \\
0 \\
0 \\
0 \\
0\end{array}$ & 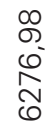 & \begin{tabular}{l}
\multirow{0}{0}{} \\
m \\
0 \\
0
\end{tabular} \\
\hline \multicolumn{2}{|c|}{ के } & & $\begin{array}{l}\bar{\xi} \\
\underline{\underline{E}}\end{array}$ & 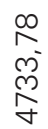 & $\begin{array}{l}0 \\
\infty \\
0 \\
\circ \\
\stackrel{5}{+}\end{array}$ & $\begin{array}{l}\infty \\
0 \\
\infty \\
0 \\
0 \\
\end{array}$ & 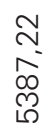 & $\begin{array}{l}\tilde{N} \\
0 \\
0 \\
0 \\
0 \\
0\end{array}$ & 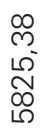 & \begin{tabular}{l} 
గ \\
\multirow{J}{*}{} \\
8
\end{tabular} & 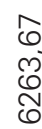 \\
\hline \multirow{5}{*}{ 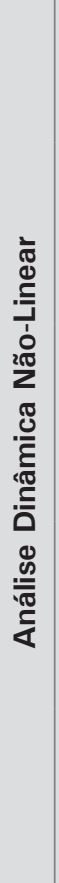 } & \multirow{5}{*}{ 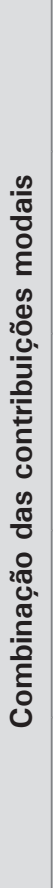 } & $\begin{array}{l}\text { م } \\
\sigma \\
\sigma\end{array}$ & $\sum_{\underline{\underline{E}}}^{\bar{\xi}}$ & $\begin{array}{l}\text { ఫ } \\
0 \\
0 \\
0 \\
m\end{array}$ & 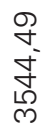 & $\begin{array}{l}\stackrel{0}{+} \\
\stackrel{+}{N} \\
\text { N }\end{array}$ & 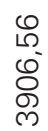 & 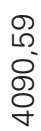 & 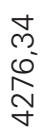 & 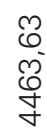 & 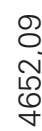 \\
\hline & & $\begin{array}{l}\sigma \\
\sigma \\
\sigma\end{array}$ & $\begin{array}{l}\bar{\xi} \\
\underline{\underline{E}}\end{array}$ & $\begin{array}{l}\infty \\
\stackrel{0}{0} \\
0^{-} \\
m \\
m \\
m\end{array}$ & 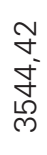 & $\begin{array}{l}\stackrel{ }{+} \\
\stackrel{+}{N} \\
\stackrel{N}{m}\end{array}$ & 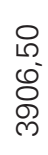 & 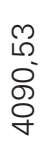 & $\begin{array}{l}\underset{\sim}{\sim} \\
\underset{\sim}{\sim} \\
\underset{\sim}{\sim}\end{array}$ & 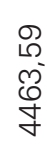 & 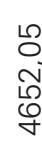 \\
\hline & & $\begin{array}{l}m \\
\sigma \\
\sigma\end{array}$ & $\sum_{\underline{\underline{E}}}^{\bar{\varepsilon}}$ & $\begin{array}{l}0 \\
0 \\
0 \\
\mathbb{0} \\
m\end{array}$ & 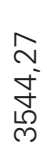 & $\underset{\stackrel{ }{\sim}}{\stackrel{\sim}{N}}$ & $\begin{array}{l}\stackrel{1}{\sim} \\
\stackrel{0}{8} \\
\stackrel{8}{\circ}\end{array}$ & 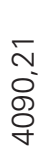 & \begin{tabular}{l}
$\infty$ \\
$\infty$ \\
$\stackrel{\circ}{\circ}$ \\
$\underset{\leftarrow}{\sim}$ \\
\multirow{\sigma}{*}{}
\end{tabular} & $\begin{array}{l}\text { o } \\
\text { ஸे } \\
0 \\
\text { 寸 }\end{array}$ & $\begin{array}{l}m \\
+ \\
i \\
0 \\
\sigma\end{array}$ \\
\hline & & $\begin{array}{l}N \\
0 \\
\sigma\end{array}$ & $\sum_{\underline{\underline{E}}}^{\bar{\xi}}$ & $\begin{array}{l}\hat{N} \\
0 \\
0 \\
\tilde{m} \\
m\end{array}$ & $\begin{array}{l}\hat{\sigma} \\
\text { m. } \\
\text { مै } \\
\text { ले }\end{array}$ & $\begin{array}{l}\infty \\
\stackrel{\infty}{N} \\
\stackrel{N}{N}\end{array}$ & 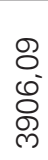 & $\begin{array}{l}\bar{\sigma} \\
\text { ᄋ̆ } \\
\text { ᄋ }\end{array}$ & \begin{tabular}{l}
$N$ \\
$\infty$ \\
$\stackrel{N}{N}$ \\
\multirow{N}{*}{} \\
\multirow{N}{*}{}
\end{tabular} & $\begin{array}{l}\text { L } \\
0 \\
\text { ர் } \\
\text { ச }\end{array}$ & 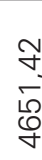 \\
\hline & & $\begin{array}{l}\text { 음 } \\
\frac{0}{0} \\
\text { 을 }\end{array}$ & $\sum_{\underline{\underline{E}}}^{\bar{\xi}}$ & مَ & 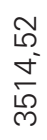 & 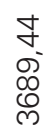 & $\begin{array}{l}0 \\
6 \\
0 \\
0 \\
\infty\end{array}$ & 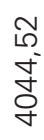 & 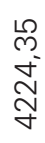 & 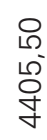 & 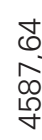 \\
\hline \multicolumn{2}{|c|}{ 定 } & & $\begin{array}{l}\bar{\varepsilon} \\
\underline{\underline{z}}\end{array}$ & $\begin{array}{l}0 \\
\text { D } \\
\infty \\
\text { D } \\
\text { N }\end{array}$ & $\begin{array}{l}\text { ' } \\
\infty \\
\text { D } \\
0 \\
\text { లి }\end{array}$ & 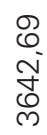 & 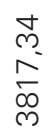 & 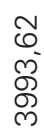 & 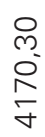 & $\begin{array}{l}\text { N } \\
\underset{\text { J }}{\text { Ð }}\end{array}$ & 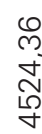 \\
\hline 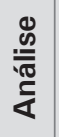 & 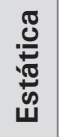 & & $\sum_{\underline{\underline{E}}}^{\bar{\varepsilon}}$ & $\begin{array}{l}m \\
\infty \\
0 \\
\stackrel{\circ}{0} \\
\stackrel{\circ}{-}\end{array}$ & 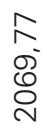 & $\begin{array}{l}\stackrel{\circ}{\sigma} \\
\stackrel{\sigma}{\sigma}\end{array}$ & $\frac{\bar{m}}{\stackrel{n}{N}}$ & 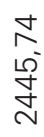 & 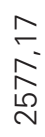 & $\begin{array}{l}\stackrel{0}{\sigma} \\
\bar{夭} \\
\sim\end{array}$ & 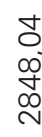 \\
\hline & $N$ & & $\underline{\xi}$ & $\begin{array}{l}\infty \\
0 \\
\stackrel{\omega}{ }\end{array}$ & $\begin{array}{l}\stackrel{0}{\sim} \\
\stackrel{-}{\sim}\end{array}$ & \begin{tabular}{l}
\multirow{0}{0}{} \\
$\stackrel{0}{\circ}$
\end{tabular} & $\frac{N}{\sigma}$ & $\begin{array}{l}0 \\
\end{array}$ & ○' & $\begin{array}{l}\mathscr{Q} \\
\nabla\end{array}$ & $\begin{array}{l}\text { J } \\
\text { M }\end{array}$ \\
\hline
\end{tabular}




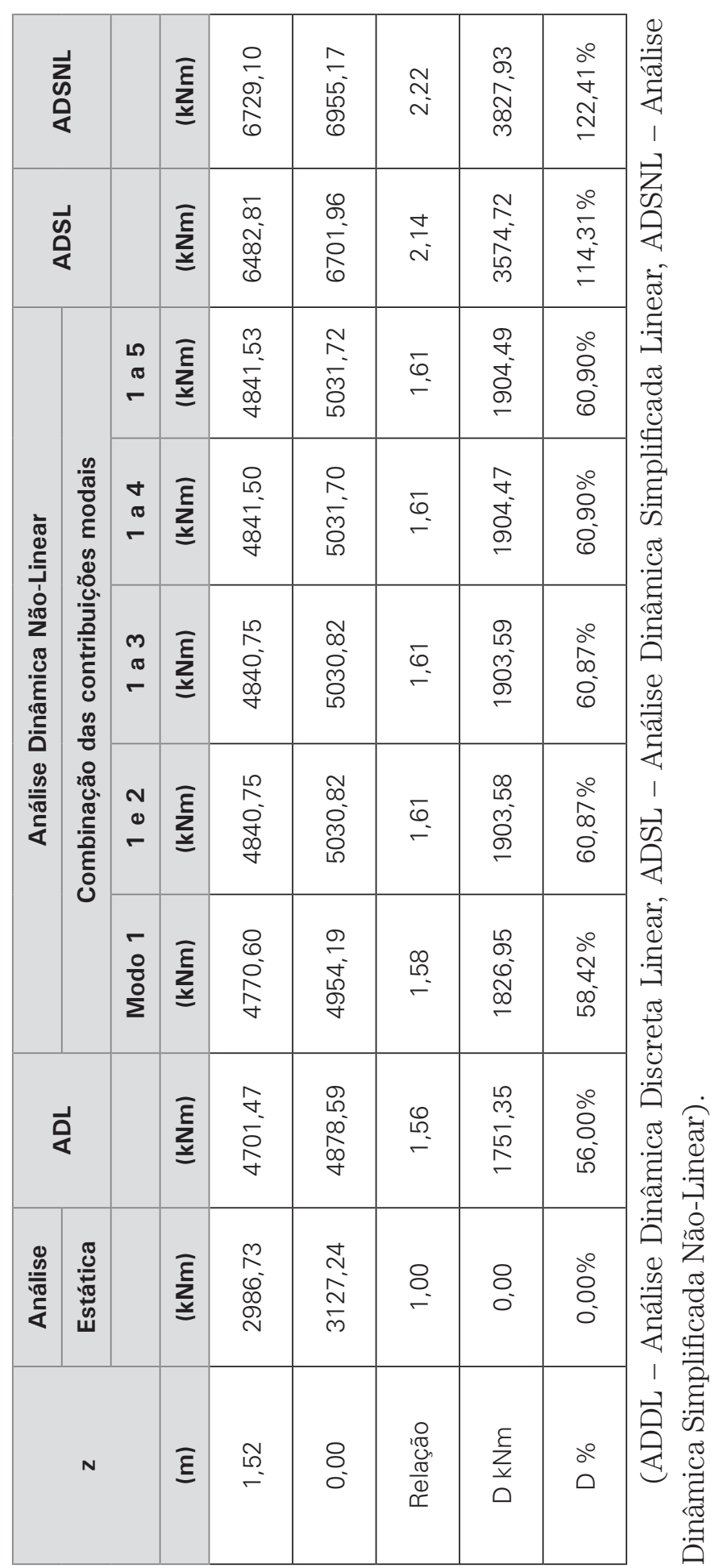


No gráfico da Figura 7.11 podem ser encontrados os resultados obtidos para a ação do vento sobre a estrutura. O traçado mais inferior corresponde ao valor do momento fletor para a análise estática, usada originalmente para dimensionar a estrutura. Os traçados superiores subsequentes correspondem aos resultados das análises dinâmicas discretas. Logo acima se encontram as curvas das análises dinâmicas simplificadas.

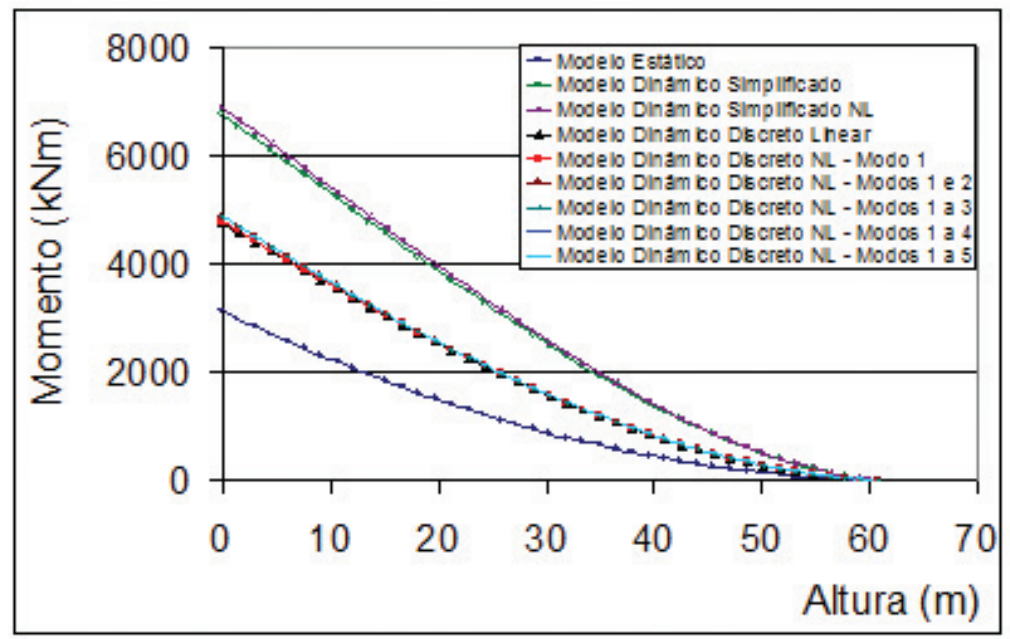

Figura 7.11 - Ação do vento na estrutura 2.

As formas modais da NBR 6123/88, a do Método dos Elementos Finitos e do método proposto constam na Figura 7.12(a).

Uma melhor aproximação à forma modal não-linear é conseguida substituindo-se o expoente da expressão (6.6) por 1,775; pois a curva definida com esse novo expoente oferece pontos mais próximos à forma modal não-linear, como pode ser observado na Figura 7.12(b).

O expoente sugerido anteriormente supera em 4,4\% o valor do recomendado pela NBR 6123/88. 


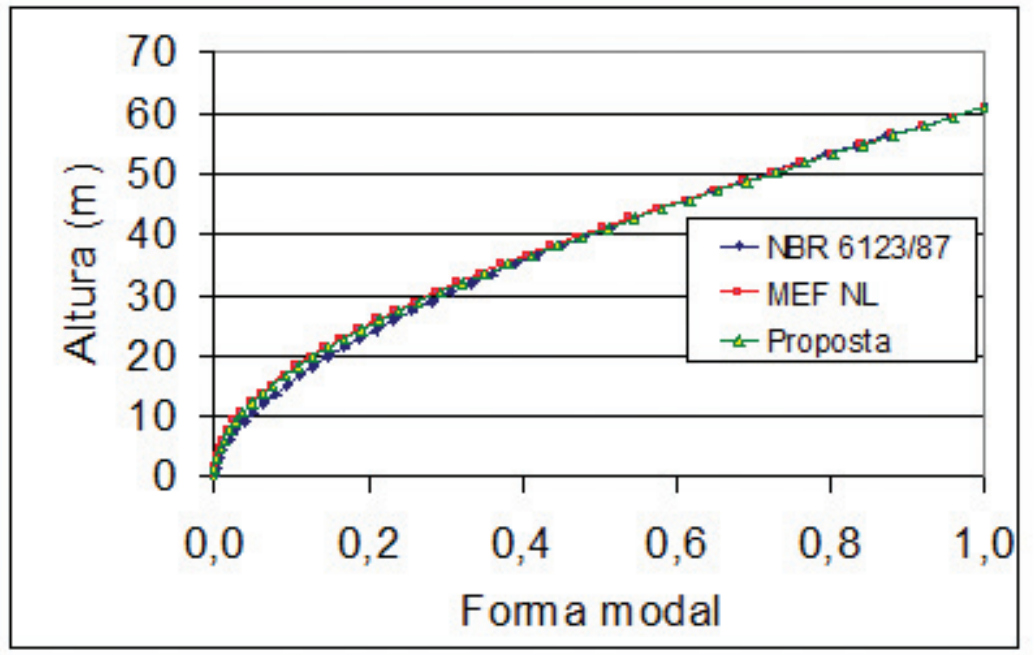

(a) expoente da forma modal: $\gamma=1,7$

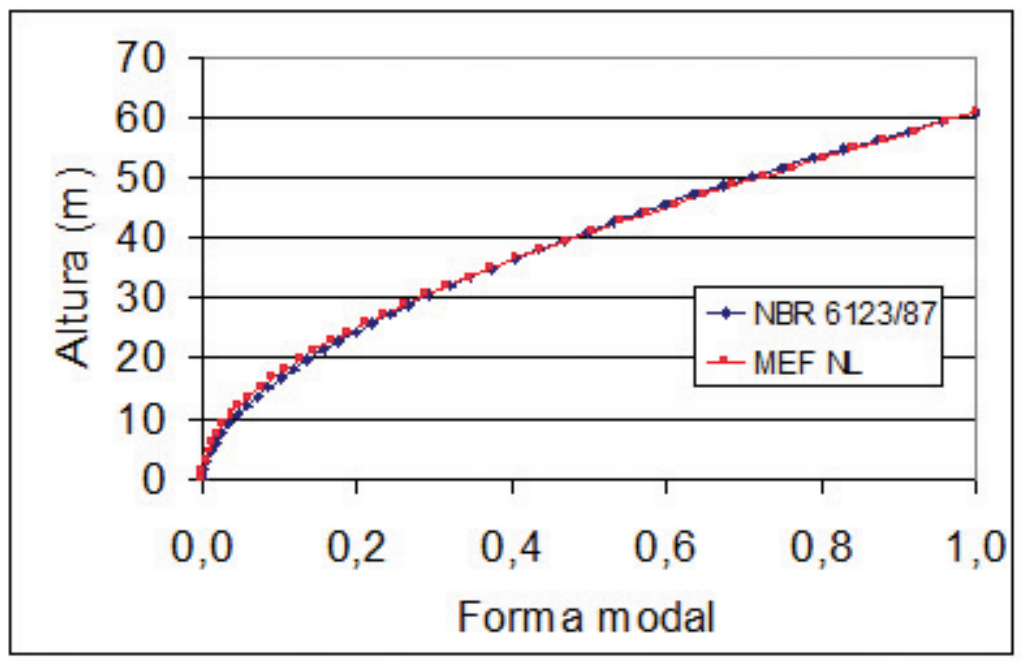

(b) expoente da forma modal: $\gamma=1,775$

Figura 7.12 - Estrutura 2 - comparativo das formas modais: (a) usada na análise, (b) sugerida. 


\subsection{ESTRUTURA 3 - POSTE DE CONCRETO ARMADO DE $40 \mathrm{M}$}

\subsubsection{Dados e geometria}

Semelhantemente às precedentes, esta é uma estrutura destinada ao suporte de sistemas irradiantes de sinal de telefonia móvel celular. É um poste de concreto armado de $40 \mathrm{~m}$ de altura e com seção transversal circular vazada de diâmetro externo de $60 \mathrm{~cm}$. As propriedades da seção mudam ao longo do comprimento devido à mudança da espessura e da variação da área de aço na seção transversal ao longo do eixo longitudinal da estrutura. o índice de esbeltez da estrutura é $\lambda=462$. A secção 15.8.1 da NBR - 6118/04 limita o índice de esbeltez a 200 de pilares isolados de concreto armado. Exceto para postes com força normal menor que $0,10 f_{c d} A_{c}$, o índice de esbeltez pode ser maior. A estrutura atende a essa condição.

O concreto usado na fabricação da estrutura teve como resistência característica à compressão igual a $45 \mathrm{MPa}$ e densidade de $2600 \mathrm{~kg} / \mathrm{m}^{3}$. O cobrimento especificado para as armaduras foi de $25 \mathrm{~mm}$ e o aço empregado na construção do poste foi o CA-50. O modulo de elasticidade secante do concreto, de $3193 \mathrm{MPa}$, foi calculado conforme a NBR 61818/03.

A estrutura possui um conjunto de antenas e acessórios, como plataforma, escada, cabos e esteiramento, com as características da Tabela 7.11. 
Tabela 7.11 - Características da estrutura 3 e dispositivos.

\begin{tabular}{|c|c|c|c|c|}
\hline Dispositivo & Área & Ca & Cota & $\begin{array}{c}\text { Peso específico, peso } \\
\text { distribuído ou peso }\end{array}$ \\
\hline Poste & $0,6 \mathrm{~m}^{2} / \mathrm{m}$ & 0,6 & de 0 a $40 \mathrm{~m}$ & $25,48 \mathrm{kN} / \mathrm{m}^{3}$ \\
\hline Escada & $0,05 \mathrm{~m}^{2} / \mathrm{m}$ & 2,0 & de 0 a $40 \mathrm{~m}$ & $0,15 \mathrm{kN} / \mathrm{m}$ \\
\hline Cabos & $0,15 \mathrm{~m}^{2} / \mathrm{m}$ & 1,2 & de 0 a $40 \mathrm{~m}$ & $0,25 \mathrm{kN} / \mathrm{m}$ \\
\hline $\begin{array}{c}\text { Plataforma e } \\
\text { suportes }\end{array}$ & $1 \mathrm{~m}^{2}$ & 2,0 & $40 \mathrm{~m}$ & $4,90 \mathrm{kN}$ \\
\hline & & & & \\
\hline Antenas & $3 \mathrm{~m}^{2}$ & 1,0 & $40 \mathrm{~m}$ & $1,88 \mathrm{kN}$ \\
\hline
\end{tabular}

(Ca indica o coeficiente de arrasto)

Como se trata de uma estrutura de concreto armado é preciso levar em conta a presença do aço no momento de inércia da seção transversal, o que deve ser feito por meio da homogeneização da seção de concreto.

Seja uma seção circular vaza de diâmetro externo D. Uma barra de aço qualquer $b_{i}$ ocupa uma posição i na seção definida por $\mathrm{Rb}_{\mathrm{i}}$ e $\theta_{\mathrm{i}}$, conforme representado na Figura 7.13.

$\mathrm{Rb}_{\mathrm{i}}$ determina a posição do centro de cada barra em relação ao centro da seção. Como todas as barras possuem o mesmo raio, por simplicidade de notação, se fará $R b_{i}=R b$, logo:

$$
\mathrm{Rb}=\frac{\mathrm{D}}{2}-\mathrm{cob}-\frac{\mathrm{d}_{\mathrm{bi}}}{2}
$$


onde cob é o cobrimento das armaduras e $\mathrm{d}_{\mathrm{bi}}$ é o diâmetro da barra i.

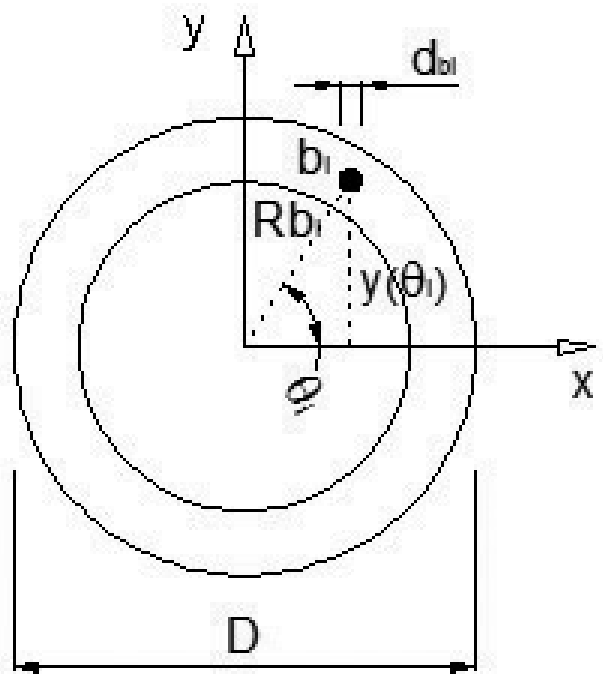

Figura 7.13 - Estrutura 3: parâmetros para homogeneização da seção.

Sendo $\theta$ a variável independente, a distância entre o centro de cada barra em relação ao eixo central de inércia da seção é $\mathrm{y}(\theta)=\operatorname{sen}(\theta) \mathrm{Rb}$. O espaçamento entre os centros de cada barra da seção foi obtido por esp $=\frac{2 \pi R_{b}}{n b}$, e a defasagem angular entre elas por $\Delta \theta=\frac{\text { esp }}{\mathrm{Rb}}$. Fazendo-se $\theta$ variar de 0 a $2 \pi$ em intervalos definidos por $\Delta \theta$, a inércia total das barras de aço em relação à seção da estrutura pôde ser obtida pelo teorema dos eixos paralelos com a expressão

$$
\mathrm{I}_{\mathrm{s}}=\sum_{\theta}^{2 \pi}\left(\frac{\pi \mathrm{d}_{\mathrm{b}}^{4}}{64}+\mathrm{y}(\theta)^{2} \frac{\pi \mathrm{d}_{\mathrm{b}}^{2}}{4}\right)
$$


O momento de inércia homogeneizado das barras de aço será, pois:

$$
\mathrm{I}_{\text {shom }}=\sum_{\theta}^{2 \pi} \mathrm{I}(\theta)\left(\frac{\mathrm{E}_{\mathrm{s}}}{\mathrm{E}_{\mathrm{csec}}}-1\right) .
$$

A parcela da inércia de concreto é $\mathrm{I}_{\text {conc }}=\mathrm{I}-\mathrm{I}_{\mathrm{s}}$, com I sendo a inércia da seção circular. A inércia total da seção homogeneizada será obtida por $\mathrm{I}_{\text {tot }}=\mathrm{I}_{\text {conc }}+\mathrm{I}_{\text {shom }}$.

Para encontrar um fator $\mathrm{F}$ que multiplique a inércia nominal da seção em termos da inércia homogeneizada da seção total de aço faz-se

$$
\mathrm{F}=1+\frac{\mathrm{I}_{\text {shom }}}{\mathrm{I}_{\text {tot }}}
$$

Os fatores de homogeneização, as propriedades estruturais e geometria da estrutura estão na Tabela 7.12. A estrutura está representada pela Figura 7.14. 
Tabela 7.12 - Estrutura 3: propriedades da estrutura e fatores de homogeneização das seções.

\begin{tabular}{|c|c|c|c|c|c|c|}
\hline Nó & Altura & $\begin{array}{c}f \\
\text { externo }\end{array}$ & Espessura & $\mathrm{nb}$ & $f b$ & $\begin{array}{c}\text { Fator de } \\
\text { inércia }\end{array}$ \\
\hline & (m) & $(\mathrm{cm})$ & $(\mathrm{cm})$ & & $(\mathrm{mm})$ & \\
\hline 41 & 40 & 60 & 10 & 20 & 13 & \multirow{9}{*}{1,0963} \\
\hline 40 & 39 & 60 & 10 & 20 & 13 & \\
\hline 39 & 38 & 60 & 10 & 20 & 13 & \\
\hline 38 & 37 & 60 & 10 & 20 & 13 & \\
\hline 37 & 36 & 60 & 10 & 20 & 13 & \\
\hline 36 & 35 & 60 & 10 & 20 & 13 & \\
\hline 35 & 34 & 60 & 10 & 20 & 13 & \\
\hline 34 & 33 & 60 & 10 & 20 & 13 & \\
\hline 33 & 32 & 60 & 10 & 20 & 13 & \\
\hline 32 & 31 & 60 & 13 & 20 & 13 & 1,0869 \\
\hline 31 & 30 & 60 & 12 & 15 & 16 & 1,0995 \\
\hline
\end{tabular}




\begin{tabular}{|c|c|c|c|c|c|c|}
\hline \multirow[t]{2}{*}{ Nó } & Altura & $\begin{array}{c}f \\
\text { externo }\end{array}$ & Espessura & \multirow[t]{2}{*}{$\mathrm{nb}$} & $f b$ & \multirow[t]{2}{*}{$\begin{array}{c}\text { Fator de } \\
\text { inércia }\end{array}$} \\
\hline & (m) & $(\mathrm{cm})$ & $(\mathrm{cm})$ & & $(\mathrm{mm})$ & \\
\hline 30 & 29 & 60 & 11 & 15 & 16 & \multirow{4}{*}{1,1029} \\
\hline 29 & 28 & 60 & 11 & 15 & 16 & \\
\hline 28 & 27 & 60 & 11 & 15 & 16 & \\
\hline 27 & 26 & 60 & 11 & 15 & 16 & \\
\hline 26 & 25 & 60 & 11 & 16 & 16 & 1,1091 \\
\hline 25 & 24 & 60 & 11 & 17 & 16 & 1,1153 \\
\hline 24 & 23 & 60 & 11 & 18 & 16 & 1,1214 \\
\hline 23 & 22 & 60 & 11 & 19 & 16 & 1,1274 \\
\hline 22 & 21 & 60 & 11 & 20 & 16 & 1,1334 \\
\hline 21 & 20 & 60 & 14 & 20 & 16 & 1,123 \\
\hline 20 & 19 & 60 & 15 & 15 & 20 & 1,1374 \\
\hline 19 & 18 & 60 & 16 & 15 & 20 & 1,1354 \\
\hline 18 & 17 & 60 & 13 & 16 & 20 & \multirow{2}{*}{1,1512} \\
\hline 17 & 16 & 60 & 13 & 16 & 20 & \\
\hline 16 & 15 & 60 & 13 & 17 & 20 & 1,1594 \\
\hline
\end{tabular}




\begin{tabular}{|c|c|c|c|c|c|c|}
\hline \multirow[t]{2}{*}{ Nó } & Altura & $\begin{array}{c}f \\
\text { externo }\end{array}$ & Espessura & \multirow[t]{2}{*}{$\mathrm{nb}$} & $f b$ & \multirow{2}{*}{$\begin{array}{c}\text { Fator de } \\
\text { inércia }\end{array}$} \\
\hline & $(\mathrm{m})$ & $(\mathrm{cm})$ & $(\mathrm{cm})$ & & $(\mathrm{mm})$ & \\
\hline 15 & 14 & 60 & 13 & 18 & 20 & 1,1675 \\
\hline 14 & 13 & 60 & 13 & 19 & 20 & \multirow{2}{*}{1,1755} \\
\hline 13 & 12 & 60 & 13 & 19 & 20 & \\
\hline 12 & 11 & 60 & 13 & 20 & 20 & 1,1833 \\
\hline 11 & 10 & 60 & 13 & 22 & 20 & 1,1987 \\
\hline 10 & 9 & 60 & 16 & 22 & 20 & 1,1889 \\
\hline 9 & 8 & 60 & 16 & 15 & 25 & 1,1961 \\
\hline 8 & 7 & 60 & 17 & 15 & 25 & 1,194 \\
\hline 7 & 6 & 60 & 14 & 16 & 25 & \multirow{2}{*}{1,2132} \\
\hline 6 & 5 & 60 & 14 & 16 & 25 & \\
\hline 5 & 4 & 60 & 14 & 17 & 25 & \multirow{3}{*}{1,2241} \\
\hline 4 & 3 & 60 & 14 & 17 & 25 & \\
\hline 3 & 2 & 60 & 14 & 17 & 25 & \\
\hline 2 & 1 & 60 & 18 & 17 & 25 & \multirow{2}{*}{1,2136} \\
\hline 1 & 0 & 60 & 18 & 17 & 25 & \\
\hline
\end{tabular}




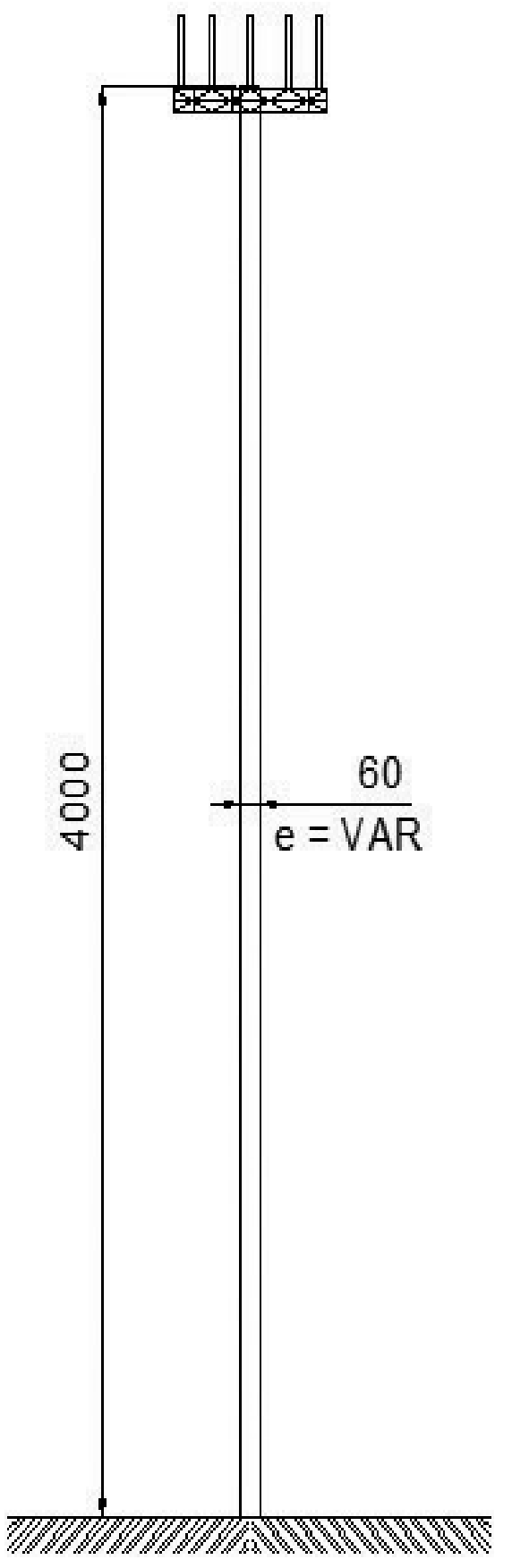

Figura 7.14 - Estrutura 3: geometria - medidas em centímetros. 


\subsubsection{Modelagem por Elementos Finitos}

A modelagem por Elementos Finitos da estrutura acompanhou os critérios utilizados na modelagem das estruturas precedentes. As seções tiveram seus momentos de inércias majorados pelos respectivos fatores de homogeneização.

A Figura 7.15 apresenta o modelo com a discretização da estrutura. Os modos de vibração e as frequências obtidas pelo Método dos Elementos Finitos são as constantes na Figura 7.16.

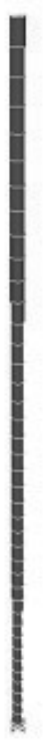

(a) $3 \mathrm{D}$
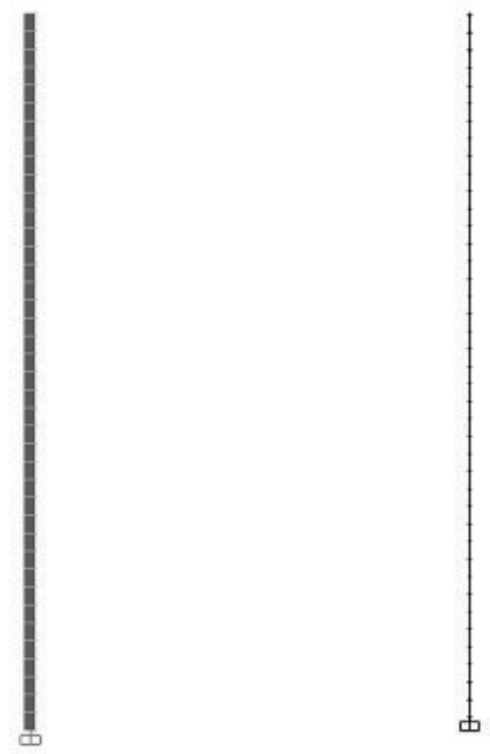

(b) Vista lateral

(a) Discretização

Figura 7.15 - Estrutura 3: modelo por Elementos Finitos. 


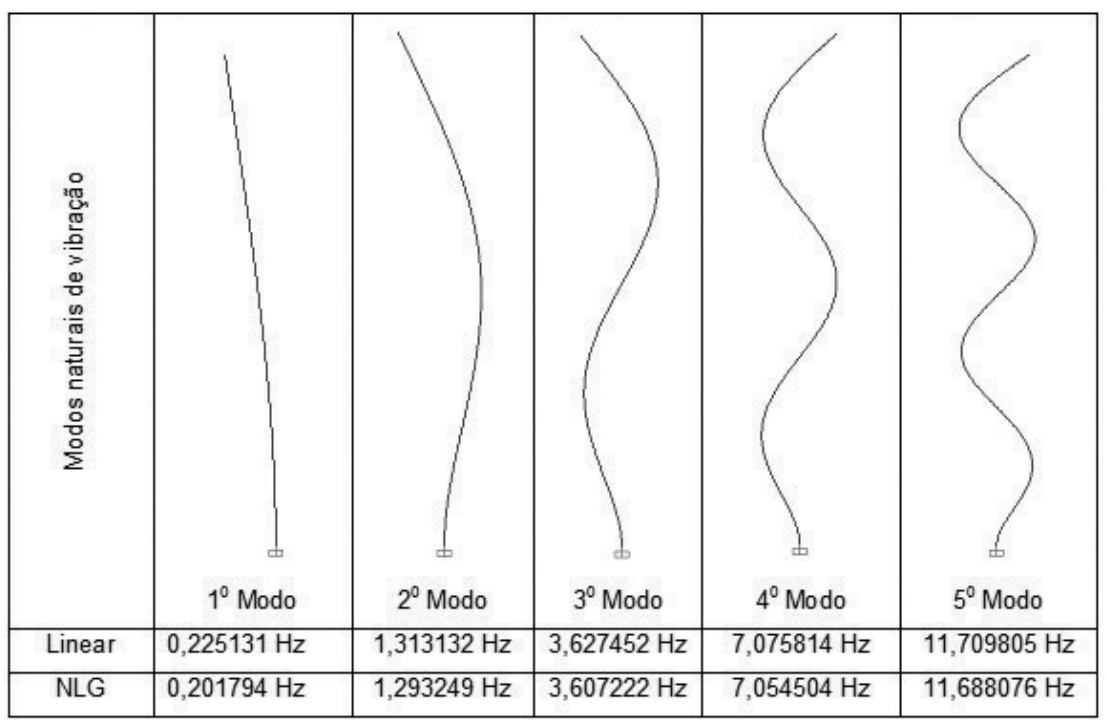

(NLG diz respeito à Não-Linearidade Geométrica)

Figura 7.16 - Estrutura 3: modos naturais de vibração.

\subsubsection{Aplicação do método proposto}

\subsubsection{Definição dos parâmetros}

Os dados utilizados na aplicação do método proposto nesta Tese foram:

- módulo de elasticidade do concreto: $\mathrm{E}=31931 \mathrm{MPa}$,

- densidade do concreto: $\rho=2600 \mathrm{~kg} / \mathrm{m}^{3}$,

- $\quad$ massa concentrada no topo: $\mathrm{m}_{0}=692 \mathrm{~kg}$;

- massa distribuída por unidade de altura: $\mathrm{m}_{\mathrm{e}}=40 \mathrm{~kg} / \mathrm{m}$.

As ordenadas de interesse ao longo da altura, a área e a inércia das seções são dadas por:

$$
\begin{aligned}
& L=L_{i}, A_{i}=\frac{\pi}{4}\left(D^{2}-d_{i}^{2}\right) \text { e } I_{i}=\frac{\pi}{64}\left(D^{4}-d_{i}^{4}\right) f_{i}, c o m \\
& i=1,2 \ldots . .40 .
\end{aligned}
$$


Nas expressões anteriores, $D$ é o diâmetro externo da estrutura, constante, nesse caso, f é o fator de homogeneização da seção e i, se refere a uma dada posição ou seção na altura.

\subsubsection{Cálculo da massa generalizada}

A massa generalizada total é obtida pelas expressões que se apresentam a seguir. A introdução dos subíndices gregos objetivam evitar a redundância de notação.

$$
\begin{aligned}
& \mathrm{M}=\mathrm{m}_{0}+\sum_{\mathrm{i}=1}^{40} \mathrm{~m}_{\mathrm{i}}, \text { com } \\
& \mathrm{m}_{\mathrm{i}}=\int_{\mathrm{i}-1}^{\mathrm{i}} \mathrm{m}_{\mathrm{\imath}} \phi(\mathrm{x})^{2} \text { sendo a massa generalizada, } \mathrm{e} \\
& \mathrm{m}_{\mathrm{\imath}}=\mathrm{A}_{\mathrm{i}} \rho+\mathrm{m}_{\mathrm{e}} \text { a massa distribuída do intervalo } \mathrm{i}, \mathrm{i} ;
\end{aligned}
$$

com $i, \imath=1,2 \ldots .40$.

\subsubsection{Cálculo da rigidez generalizada}

A parcela da rigidez generalizada relativa à rigidez geométrica foi obtida por:

$$
\begin{aligned}
& \mathrm{K}_{\mathrm{g}}=\sum_{\mathrm{i}=1}^{40} \mathrm{~K}_{\mathrm{gi}}, \text { com } \\
& \mathrm{K}_{\mathrm{gi}}=\int_{\mathrm{i}-1}^{\mathrm{i}}\left[\mathrm{F}_{0}+\sum_{\mathrm{i}+1}^{40} \mathrm{~F}_{\mathrm{i}}+\mathrm{m}_{\mathrm{i}}\left(\mathrm{L}_{\mathrm{i}}-\mathrm{x}\right) \mathrm{g}\right]\left(\frac{\mathrm{d}}{\mathrm{dx}} \phi(\mathrm{x})\right)^{2} \mathrm{dx}, \\
& \mathrm{F}_{\mathrm{i}}=\int_{\mathrm{i}-1}^{\mathrm{i}} \mathrm{m}_{\mathrm{l}} \mathrm{gdx}
\end{aligned}
$$


e

$$
\mathrm{F}_{0}=\mathrm{m}_{0} \mathrm{~g}
$$

A rigidez elástica generalizada, por sua vez, foi encontrada por:

$$
\begin{aligned}
& \mathrm{K}_{0}=\sum_{\mathrm{i}=1}^{40} \mathrm{~K}_{0 \mathrm{i}}, \mathrm{com} \\
& \mathrm{K}_{0 \mathrm{i}}=\int_{\mathrm{i}-1}^{\mathrm{i}} \mathrm{EI}_{\mathrm{i}}\left(\frac{\mathrm{d}}{\mathrm{dx}} \phi(\mathrm{x})\right)^{2} \mathrm{dx}, \text { com } \mathrm{i}=1,2 \ldots .40 .
\end{aligned}
$$

Com isso, a rigidez generalizada da estrutura pôde ser calculada fazendo $\mathrm{K}=\mathrm{K}_{0}-\mathrm{K}_{\mathrm{g}}$, considerando o esforço normal de compressão positivo.

\subsubsection{Cálculo da frequência}

A frequência natural da estrutura relativa ao primeiro modo de vibração, considerando a rigidez geométrica, é de $0,214575 \mathrm{~Hz}$, enquanto que para o modelo linear é de 0,237367 Hz.

\subsubsection{Ação do vento}

\subsubsection{Forças estáticas devidas ao vento}

Os parâmetros empregados na determinação das forças estáticas devidas a ação do vento foram: fator topográfico $S_{1}=1,0$; fator de rugosidade do terreno $\mathrm{S}_{2}$ correspondente à categoria $\mathrm{IV}$, classe $\mathrm{B}$, com os parâmetros $\mathrm{p}=0,125, \mathrm{~b}=0,85$ e $\mathrm{F}_{\mathrm{r}}=0,98$; fator estatístico $\mathrm{S}_{3}=1,1$; velocidade básica do vento $\mathrm{V}_{0}=35 \mathrm{~m} / \mathrm{s}$. 


\subsubsection{Resposta dinâmica pelo modelo simplificado da NBR 6123/88}

Os parâmetros usados na determinação da resposta dinâmica pelo modelo contínuo simplificado da NBR 6123/88 foram os seguintes: largura da edificação 0,6 m; altura da edificação 40 m; categoria do terreno, velocidade básica do vento e fatores estatísticos como descrito no item anterior.

A frequência fundamental foi obtida fazendo $T_{1}=0,015 \mathrm{~h}(\mathrm{Ta}-$ bela 6.4), $\log \mathrm{f}_{1}=1,666667 \mathrm{~Hz}$. A forma modal foi obtida pela expressão (6.6) com g igual a 1,7. A relação adimensional $\mathrm{Vp} /\left(\mathrm{f}_{1} \mathrm{~L}\right)$ é igual a 0,009 , o que leva, com uma taxa crítica de amortecimento $\zeta$ igual a 0,01, a um coeficiente de amplificação dinâmica $\xi$ de 1,611.

O modelo simplificado não-linear foi calculado com o coeficiente de amplificação dinâmica, obtido com a frequência do modelo discreto não-linear, que será descrito adiante.

\subsubsection{Resposta dinâmica pelo modelo discreto da NBR 6123/88}

A resposta dinâmica pelo modelo dinâmico discreto linear foi calculada com base na frequência $0,225131 \mathrm{~Hz}$. A relação adimensional Vp/(f $\left.\mathrm{f}_{1} \mathrm{~L}\right)$, nesse caso, é 0,066, e o fator de amplificação dinâmica $\xi$ é igual a 2,313, para o amortecimento crítico de 0,01. A frequência fundamental da estrutura com a inclusão da não-linearidade geométrica é $0,201796 \mathrm{~Hz}$, o que leva a um coeficiente de amplificação dinâmica $\xi$ de 2,516. Para os modos de 2 a 5 os fatores de amplificação dinâmica, dadas as frequências apresentadas na Figura 7.16, são, respectivamente: 1,$686 ; 1,400 ; 1,354 ; 1,236$.

\subsubsection{Análise dos resultados}

Os esforços normais em relação às alturas definidas na discretização da estrutura 3, obtidos pela solução matemática do método proposto, e pelo Método dos Elementos Finitos, estão na Tabela 7.13. 


\begin{tabular}{|c|c|c|c|c|c|c|c|c|}
\hline \multirow{2}{*}{ 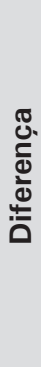 } & do & 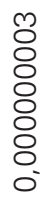 & \begin{tabular}{l} 
\} $\\
{8} \\
{8} \\
{8} \\
{8} \\
{8} \\
{8} \\
{\circ}$ & $\begin{array}{l}\text { ㅇ } \\
8 \\
8 \\
8 \\
8 \\
8 \\
\circ \\
\circ\end{array}$ & $\begin{array}{l}\text { \. } \\
8 \\
8 \\
8 \\
8 \\
8 \\
\circ\end{array}$ & $\begin{array}{l}\text { m } \\
8 \\
8 \\
8 \\
8 \\
8 \\
8 \\
\circ\end{array}$ & $\begin{array}{l}\text { ঠ } \\
8 \\
8 \\
8 \\
8 \\
8 \\
8 \\
\circ\end{array}$ & $\begin{array}{l}\text { ஜ } \\
8 \\
8 \\
8 \\
8 \\
8 \\
8 \\
0\end{array}$ \\
\hline & $\stackrel{\text { \& }}{<}$ & $\begin{array}{l}\circ \\
8 \\
8 \\
\circ \\
\circ\end{array}$ & $\begin{array}{l}\text { ㅇ } \\
8 \\
8 \\
\circ\end{array}$ & $\begin{array}{l}\circ \\
8 \\
8 \\
\circ \\
\circ\end{array}$ & $\begin{array}{l}\circ \\
\text { ᄋ } \\
\circ \\
0\end{array}$ & $\begin{array}{l}\text { ㅇ } \\
8 \\
\circ \\
0\end{array}$ & $\begin{array}{l}8 \\
8 \\
8 \\
\circ\end{array}$ & $\begin{array}{l}8 \\
8 \\
8 \\
\circ \\
\circ\end{array}$ \\
\hline 出 & $\bar{z}_{\underline{\underline{z}}}$ & 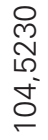 & $\begin{array}{l}m \\
\infty \\
m \\
\tilde{m} \\
\sigma \\
\sigma\end{array}$ & 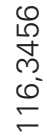 & 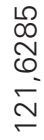 & 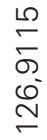 & 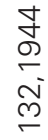 & 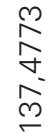 \\
\hline $\begin{array}{l}\stackrel{0}{0} \\
\text { : } \\
\frac{0}{0} \\
\text { ㅇ }\end{array}$ & $\bar{z}_{\underline{z}}$ & 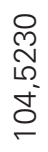 & $\begin{array}{l}m \\
\infty \\
\infty \\
\tilde{\sigma} \\
\sigma \\
\sigma\end{array}$ & $\begin{array}{l}0 \\
\stackrel{0}{ } \\
\text { m } \\
0^{\circ} \\
\leftarrow\end{array}$ & 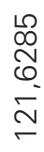 & 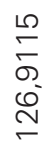 & 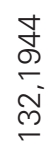 & \begin{tabular}{l}
$\stackrel{m}{N}$ \\
\multirow{\delta}{*}{} \\
m
\end{tabular} \\
\hline- & छ్ & $\stackrel{\square}{\square}$ & $\stackrel{\infty}{\sim}$ & 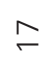 & $\ddot{\sigma}$ & $\stackrel{\llcorner}{\circ}$ & $\stackrel{\nabla}{ }$ & $\stackrel{m}{2}$ \\
\hline \multirow{2}{*}{ 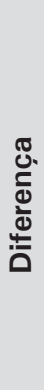 } & ১0 & $\begin{array}{l}8 \\
8 \\
8 \\
8 \\
8 \\
8 \\
8 \\
\circ \\
\circ\end{array}$ & $\begin{array}{l}\text { m } \\
8 \\
8 \\
8 \\
8 \\
8 \\
8 \\
0\end{array}$ & $\begin{array}{l}\text { ঠ } \\
\text { ᄋ } \\
8 \\
8 \\
8 \\
8 \\
\circ \\
\circ\end{array}$ & $\begin{array}{l}\text { L } \\
\text { ᄋ } \\
8 \\
8 \\
8 \\
8 \\
8 \\
\circ \\
\circ\end{array}$ & $\begin{array}{l}\text { ㅇ } \\
8 \\
8 \\
8 \\
8 \\
8 \\
8 \\
8 \\
0\end{array}$ & $\begin{array}{l}0 \\
8 \\
8 \\
8 \\
8 \\
8 \\
8 \\
0 \\
0\end{array}$ & $\begin{array}{l}0 \\
8 \\
8 \\
8 \\
8 \\
8 \\
8 \\
8 \\
0\end{array}$ \\
\hline & $\stackrel{\mathscr{0}}{\stackrel{0}{\alpha}}$ & $\begin{array}{l}8 \\
8 \\
8 \\
\circ\end{array}$ & $\begin{array}{l}8 \\
8 \\
8 \\
\circ \\
\circ\end{array}$ & $\begin{array}{l}8 \\
8 \\
8 \\
\circ\end{array}$ & $\begin{array}{l}\circ \\
8 \\
8 \\
\circ \\
0\end{array}$ & $\begin{array}{l}8 \\
8 \\
8 \\
\circ\end{array}$ & $\begin{array}{l}8 \\
8 \\
8 \\
\circ\end{array}$ & $\begin{array}{l}\text { ㅇ } \\
8 \\
\circ \\
\circ\end{array}$ \\
\hline 岀 & $\bar{z}_{\underline{\underline{z}}}$ & $\begin{array}{l}\frac{0}{\infty} \\
\frac{1}{0} \\
0^{-}\end{array}$ & 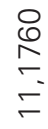 & 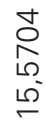 & 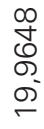 & 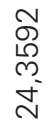 & $\begin{array}{l}\stackrel{\llcorner}{N} \\
\stackrel{\omega}{N} \\
\stackrel{\infty}{N} \\
\stackrel{\infty}{N}\end{array}$ & 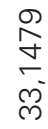 \\
\hline $\begin{array}{l}\text { 요 } \\
\text { : } \\
\text { 은 } \\
\text { ㄴ }\end{array}$ & $\bar{z}_{\underline{\underline{z}}}$ & $\begin{array}{l}0 \\
\infty \\
0 \\
0 \\
0\end{array}$ & 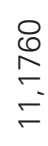 & 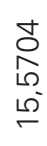 & 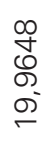 & 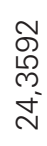 & $\begin{array}{l}\stackrel{L}{N} \\
\stackrel{1}{\rho} \\
\stackrel{\infty}{\infty} \\
\sim\end{array}$ & 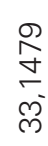 \\
\hline 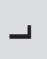 & $\bar{\xi}$ & 아 & ল & ల & $\hat{m}$ & ले & $\stackrel{\llcorner}{\aleph}$ & ঙ \\
\hline
\end{tabular}
\end{tabular}




\begin{tabular}{|c|c|c|c|c|c|c|c|c|c|}
\hline \multirow{2}{*}{ 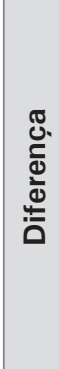 } & ১ீ & $\begin{array}{l}\text { ஜ } \\
8 \\
8 \\
8 \\
8 \\
8 \\
\circ\end{array}$ & $\begin{array}{l}\text { 尺े } \\
8 \\
8 \\
8 \\
8 \\
\circ \\
\circ\end{array}$ & $\begin{array}{l}\text { ○े } \\
8 \\
8 \\
8 \\
8 \\
8 \\
\circ \\
\circ\end{array}$ & $\begin{array}{l}\text { ঠे } \\
8 \\
8 \\
8 \\
8 \\
8 \\
\circ \\
\circ\end{array}$ & $\begin{array}{l}\text { ஜ } \\
8 \\
8 \\
8 \\
8 \\
8 \\
\circ \\
\circ \\
0\end{array}$ & $\begin{array}{l}5 \\
8 \\
8 \\
8 \\
8 \\
8 \\
8 \\
\circ\end{array}$ & $\begin{array}{l}\text { 尺े } \\
8 \\
8 \\
8 \\
8 \\
8 \\
\circ \\
\circ\end{array}$ & $\begin{array}{l}\text { ஜ } \\
8 \\
8 \\
8 \\
8 \\
8 \\
8 \\
\circ\end{array}$ \\
\hline & $\stackrel{n}{\stackrel{2}{\alpha}}$ & $\begin{array}{l}8 \\
8 \\
8 \\
0\end{array}$ & $\begin{array}{l}8 \\
8 \\
8 \\
\circ\end{array}$ & $\begin{array}{l}\text { ㅇ } \\
8 \\
\circ \\
\circ\end{array}$ & $\begin{array}{l}\circ \\
8 \\
8 \\
\circ \\
\circ\end{array}$ & $\begin{array}{l}8 \\
8 \\
8 \\
\circ \\
0\end{array}$ & $\begin{array}{l}8 \\
8 \\
8 \\
\circ\end{array}$ & $\begin{array}{l}8 \\
8 \\
8 \\
0\end{array}$ & $\begin{array}{l}\circ \\
8 \\
\circ \\
\circ \\
\circ\end{array}$ \\
\hline 岃 & $\underline{\underline{z}}$ & 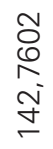 & 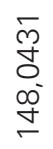 & 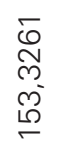 & 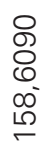 & $\begin{array}{l}\text { ల్ } \\
\text { ले } \\
0 \\
0 \\
0\end{array}$ & $\begin{array}{l}\hat{N} \\
0 \\
0 \\
\stackrel{-}{0} \\
\stackrel{-}{-}\end{array}$ & 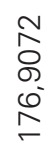 & \begin{tabular}{l} 
m \\
\multirow{L}{L}{} \\
\multirow{\sigma}{*}{} \\
$\infty$ \\
$\infty$ \\
$\infty$
\end{tabular} \\
\hline 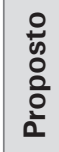 & $\underset{\underline{\underline{\Sigma}}}{\overline{\underline{z}}}$ & 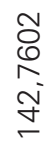 & 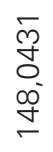 & $\begin{array}{l}\bar{\Theta} \\
\text { N } \\
\tilde{N} \\
\stackrel{N}{\sigma}\end{array}$ & 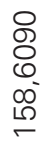 & 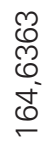 & $\begin{array}{l}\hat{m} \\
0 \\
0 \\
\stackrel{\circ}{0} \\
\stackrel{-}{-}\end{array}$ & 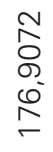 & \begin{tabular}{l} 
m \\
\multirow{5}{S}{} \\
\multirow{5}{*}{} \\
$\infty$ \\
$\infty$ \\
-
\end{tabular} \\
\hline$\lrcorner$ & $\widehat{\underline{\xi}}$ & $\stackrel{\sim}{\leftarrow}$ & $\mp$ & $\stackrel{\circ}{\circ}$ & $\sigma$ & $\infty$ & $\wedge$ & 0 & مصا \\
\hline \multirow{2}{*}{ 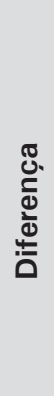 } & ১゚ & $\begin{array}{l}0 \\
8 \\
8 \\
8 \\
8 \\
8 \\
\circ \\
0\end{array}$ & $\begin{array}{l}8 \\
8 \\
8 \\
8 \\
8 \\
8 \\
\circ \\
0\end{array}$ & $\begin{array}{l}0 \\
8 \\
8 \\
8 \\
8 \\
8 \\
8 \\
8 \\
0\end{array}$ & $\begin{array}{l}\text { 으 } \\
\text { 8̊ } \\
8 \\
8 \\
8 \\
8\end{array}$ & 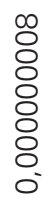 & $\begin{array}{l}5 \\
8 \\
8 \\
8 \\
8 \\
8 \\
8 \\
0\end{array}$ & $\begin{array}{l}0 \\
8 \\
8 \\
8 \\
8 \\
8 \\
8 \\
0\end{array}$ & $\begin{array}{l}\text { ㅇ } \\
8 \\
8 \\
8 \\
8 \\
8 \\
8 \\
\circ\end{array}$ \\
\hline & $\stackrel{n}{\frac{0}{\alpha}}$ & $\begin{array}{l}8 \\
8 \\
8 \\
0\end{array}$ & $\begin{array}{l}8 \\
8 \\
\circ \\
\circ\end{array}$ & $\begin{array}{l}8 \\
8 \\
\circ \\
\circ \\
\circ\end{array}$ & $\begin{array}{l}8 \\
8 \\
8 \\
\circ\end{array}$ & $\begin{array}{l}8 \\
8 \\
8 \\
\circ\end{array}$ & $\begin{array}{l}8 \\
8 \\
8 \\
\circ\end{array}$ & $\begin{array}{l}8 \\
8 \\
8 \\
\circ\end{array}$ & $\begin{array}{l}8 \\
8 \\
8 \\
0\end{array}$ \\
\hline 出 & $\underline{\underline{z}}$ & $\begin{array}{l}\stackrel{M}{N} \\
\text { D } \\
\stackrel{n}{m}\end{array}$ & 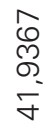 & $\begin{array}{l}\underset{\check{m}}{m} \\
\underset{\sigma}{\sigma}\end{array}$ & 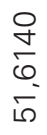 & $\begin{array}{l}\infty \\
0 \\
\overline{0} \\
0^{-} \\
\sigma^{\circ}\end{array}$ & $\begin{array}{l}\text { m } \\
\underset{n}{n} \\
\bar{\sigma}\end{array}$ & $\begin{array}{l}\text { 尺 } \\
\text { ป } \\
0 \\
0 \\
0\end{array}$ & 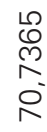 \\
\hline $\begin{array}{l}\text { 옹 } \\
\text { : } \\
\text { 응 } \\
\text { 는 }\end{array}$ & $\underset{\underline{\underline{z}}}{\overline{\underline{z}}}$ & 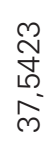 & $\begin{array}{l}\hat{0} \\
\text { ల్ } \\
\dot{\gamma}\end{array}$ & 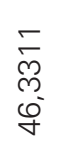 & 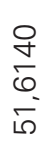 & $\begin{array}{l}\infty \\
0 \\
0 \\
0 \\
0^{-} \\
0^{\circ}\end{array}$ & $\begin{array}{l}m \\
\stackrel{n}{N} \\
\underset{\sigma}{\sigma}\end{array}$ & $\begin{array}{l}\text { D } \\
\text { Oे } \\
0 \\
0 \\
0\end{array}$ & $\begin{array}{l}\text { D } \\
0 \\
\text { N } \\
0 \\
0\end{array}$ \\
\hline- & $\widehat{\underline{\xi}}$ & $m$ & ๗ె & $\bar{m}$ & 이 & $\stackrel{N}{N}$ & $\stackrel{\infty}{\sim}$ & $\lesssim$ & $\stackrel{\bullet}{\sim}$ \\
\hline
\end{tabular}




\begin{tabular}{|c|c|c|c|c|c|c|c|}
\hline 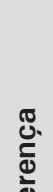 & ১̊ & 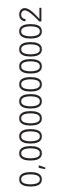 & 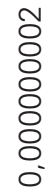 & $\begin{array}{l}5 \\
8 \\
8 \\
8 \\
8 \\
8 \\
8 \\
\end{array}$ & $\begin{array}{l}5 \\
8 \\
8 \\
8 \\
8 \\
8 \\
8 \\
\circ\end{array}$ & $\begin{array}{l}\overline{8} \\
8 \\
8 \\
8 \\
8 \\
8 \\
\circ \\
\circ\end{array}$ & \\
\hline & $\stackrel{\mathscr{0}}{\text { \& }}$ & $\begin{array}{l}8 \\
8 \\
8 \\
\circ\end{array}$ & $\begin{array}{l}8 \\
8 \\
8 \\
\circ\end{array}$ & $\begin{array}{l}\text { ○ } \\
8 \\
\circ \\
\circ\end{array}$ & $\begin{array}{l}\text { ○ } \\
\text { ○ } \\
\text { ○. }\end{array}$ & $\begin{array}{l}8 \\
8 \\
\circ \\
\circ \\
\circ\end{array}$ & \\
\hline 岀 & $\underline{\underline{z}}$ & $\begin{array}{l}\text { m } \\
\text { ○ } \\
0 \\
\infty \\
\infty \\
-\end{array}$ & 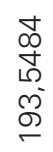 & 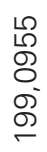 & 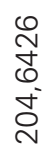 & 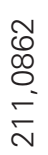 & \\
\hline 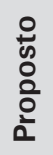 & $\underline{\sum_{z}}$ & $\begin{array}{l}m \\
\delta \\
\varnothing \\
\infty \\
\infty \\
\infty\end{array}$ & 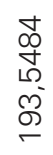 & 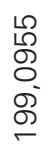 & 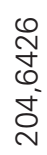 & 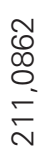 & \\
\hline- & $\overline{\underline{\xi}}$ & $\nabla$ & $m$ & $\sim$ & - & 0 & \\
\hline 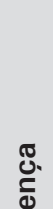 & ১̊ & $\begin{array}{l}10 \\
8 \\
8 \\
8 \\
8 \\
8 \\
8 \\
\circ \\
0\end{array}$ & 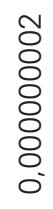 & $\begin{array}{l}\text { ஜ } \\
8 \\
8 \\
8 \\
8 \\
8 \\
\circ \\
\circ\end{array}$ & $\begin{array}{l}\text { ஜ } \\
8 \\
8 \\
8 \\
8 \\
8 \\
8 \\
\circ\end{array}$ & 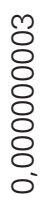 & $\begin{array}{l}\text { m } \\
8 \\
8 \\
8 \\
8 \\
8 \\
8 \\
\circ\end{array}$ \\
\hline$\Delta$ & $\stackrel{0}{\stackrel{0}{\alpha}}$ & $\begin{array}{l}\circ \\
8 \\
\bigcirc \\
\circ \\
0\end{array}$ & $\begin{array}{l}8 \\
8 \\
8 \\
0\end{array}$ & $\begin{array}{l}8 \\
\text { ○ } \\
\circ \\
\circ\end{array}$ & $\begin{array}{l}8 \\
8 \\
8 \\
\circ \\
\end{array}$ & $\begin{array}{l}8 \\
8 \\
8 \\
\circ \\
\circ\end{array}$ & $\begin{array}{l}8 \\
8 \\
8 \\
0\end{array}$ \\
\hline 岀 & $\underset{\text { 方 }}{ }$ & $\begin{array}{l}\bar{m} \\
\stackrel{+}{\sim} \\
\stackrel{N}{N}\end{array}$ & 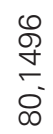 & \begin{tabular}{l}
$\widetilde{N}$ \\
$\stackrel{1}{ }$ \\
$\infty$ \\
\multirow{J}{-}{}
\end{tabular} & $\begin{array}{l}\infty \\
\sim \\
0 \\
\llcorner \\
\infty \\
\infty \\
\infty\end{array}$ & 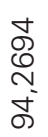 & $\begin{array}{l}\circ \\
\varnothing \\
\text { の } \\
\infty \\
\text { の }\end{array}$ \\
\hline 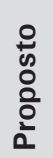 & $\underline{\underline{z}}$ & $\begin{array}{l}\bar{m} \\
\stackrel{y}{N} \\
\stackrel{N}{N}\end{array}$ & $\begin{array}{l}\mathscr{Q} \\
\stackrel{D}{+} \\
\stackrel{-}{\circ} \\
\infty\end{array}$ & 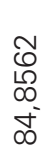 & 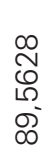 & 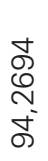 & $\begin{array}{l}\circ \\
0 \\
\text { o } \\
\infty \\
\text { o }\end{array}$ \\
\hline$\lrcorner$ & $\overline{\mathrm{E}}$ & $\stackrel{\llcorner}{\sim}$ & $\stackrel{\Xi}{\sim}$ & $\stackrel{M}{N}$ & $\approx$ & $\bar{\sim}$ & $\stackrel{৩}{\sim}$ \\
\hline
\end{tabular}


A frequência do primeiro modo de vibração obtida pelo método proposto, como descrito no item 7.4 .3 foi de $0,215715 \mathrm{~Hz}$ e, pelo Método dos Elementos Finitos, foi de 0,201794 Hz, uma diferença de 6,9\%. Pela NBR 6123/88 essa frequência ficou em 1,666667 Hz. No cálculo simplificado do método proposto, utilizando a Eq. (4.21), com as propriedades geométricas da estrutura de forma ponderada, encontra-se o valor de $0,189377 \mathrm{~Hz}$, o que representa uma diferença de 13,91\% em relação ao cálculo exato do método; de 6,15\% em relação ao MEF e de $88,64 \%$ em à NBR 6123/88.

Acompanhando os resultados já encontrados, os valores críticos da ação do vento encontram-se na comparação entre o modelo estático e o modelo dinâmico discreto simplificado não-linear. A diferença do momento fletor máximo na estrutura, entre essas duas análises, atinge um valor de 61,19\%.

Nas análises discretas não-lineares segue-se com uma pequena influência da contribuição dos modos de vibração acima do fundamental. Quando computadas as contribuições do vento médio e as flutuações do $1^{\circ}$ ao $5^{\circ}$ modo surge uma diferença de 0,006\% em relação à combinação que leva em conta o vento médio e apenas a flutuação do primeiro modo, sendo, portanto, desprezível. Nesse caso, as flutuações resultantes do primeiro modo de vibração são as que aportam maior contribuição na resposta da estrutura, com $68 \%$ do valor total.

A diferença entre a análise discreta não-linear e análise das forças estáticas é de 29,07\%. Os resultados das diferentes análises para a determinação da ação do vento podem ser encontradas na Tabela 7.14.

Analisando os resultados dos modelos discretos, linear e não-linear, observa-se, para o modelo não-linear, uma diminuição, na frequência do primeiro modo de vibração, de 10,34\% e uma elevação do fator de amplificação dinâmica de 10,57\% .

A Tabela 7.15 resume o valor dos momentos na estrutura devidos à ação do vento das análises descritas anteriormente, comparando-as ao modelo estático. 
Tabela 7.14 - Estrutura 3: momentos da análise discreta nãolinear.

\begin{tabular}{|c|c|c|c|c|c|c|}
\hline \multirow{2}{*}{$\mathbf{z}$} & \multirow{2}{*}{$\begin{array}{l}\text { Vento } \\
\text { Médio }\end{array}$} & \multicolumn{5}{|c|}{ Flutuações } \\
\hline & & Modo1 & Modo 2 & Modo 3 & Modo 4 & Modo 5 \\
\hline (m) & (kNm) & (kNm) & (kNm) & (kNm) & (kNm) & $(\mathrm{kNm})$ \\
\hline 40 & 0,00 & 0,00 & 0,00 & 0,00 & 0,00 & 0,00 \\
\hline 39 & 2,19 & 1,93 & 0,00 & 0,32 & 0,09 & 0,16 \\
\hline 38 & 4,62 & 4,76 & 0,01 & 0,75 & 0,20 & 0,36 \\
\hline 37 & 7,29 & 8,47 & 0,02 & 1,27 & 0,33 & 0,55 \\
\hline 36 & 10,20 & 13,02 & 0,02 & 1,83 & 0,45 & 0,71 \\
\hline 35 & 13,35 & 18,38 & 0,03 & 2,40 & 0,56 & 0,81 \\
\hline 34 & 16,73 & 24,52 & 0,04 & 2,95 & 0,64 & 0,83 \\
\hline 33 & 20,34 & 31,40 & 0,05 & 3,44 & 0,68 & 0,76 \\
\hline 32 & 24,18 & 38,99 & 0,06 & 3,86 & 0,69 & 0,61 \\
\hline 31 & 28,24 & 47,25 & 0,07 & 4,18 & 0,65 & 0,39 \\
\hline 30 & 32,52 & 56,22 & 0,08 & 4,37 & 0,57 & 0,13 \\
\hline 29 & 37,02 & 65,90 & 0,09 & 4,42 & 0,44 & 0,15 \\
\hline 28 & 41,73 & 76,23 & 0,10 & 4,32 & 0,29 & 0,41 \\
\hline
\end{tabular}




\begin{tabular}{|c|c|c|c|c|c|c|}
\hline \multirow{2}{*}{ z } & \multirow{2}{*}{$\begin{array}{l}\text { Vento } \\
\text { Médio }\end{array}$} & \multicolumn{5}{|c|}{ Flutuações } \\
\hline & & Modo1 & Modo 2 & Modo 3 & Modo 4 & Modo 5 \\
\hline (m) & (kNm) & (kNm) & (kNm) & (kNm) & (kNm) & $(\mathrm{kNm})$ \\
\hline 27 & 46,65 & 87,14 & 0,10 & 4,08 & 0,12 & 0,62 \\
\hline 26 & 51,78 & 98,60 & 0,11 & 3,70 & 0,06 & 0,75 \\
\hline 25 & 57,11 & 110,57 & 0,11 & 3,20 & 0,24 & 0,80 \\
\hline 24 & 62,64 & 123,04 & 0,12 & 2,59 & 0,40 & 0,75 \\
\hline 23 & 68,37 & 135,95 & 0,12 & 1,90 & 0,53 & 0,61 \\
\hline 22 & 74,29 & 149,29 & 0,12 & 1,14 & 0,62 & 0,40 \\
\hline 21 & 80,39 & 163,01 & 0,12 & 0,33 & 0,68 & 0,14 \\
\hline 20 & 86,68 & 177,09 & 0,12 & 0,48 & 0,68 & 0,15 \\
\hline 19 & 93,15 & 191,54 & 0,12 & 1,28 & 0,64 & 0,42 \\
\hline 18 & 99,80 & 206,34 & 0,11 & 2,03 & 0,55 & 0,65 \\
\hline 17 & 106,62 & 221,49 & 0,11 & 2,69 & 0,42 & 0,80 \\
\hline 16 & 113,60 & 236,94 & 0,10 & 3,24 & 0,25 & 0,85 \\
\hline 15 & 120,75 & 252,64 & 0,09 & 3,66 & 0,07 & 0,81 \\
\hline 14 & 128,05 & 268,55 & 0,07 & 3,95 & 0,12 & 0,67 \\
\hline
\end{tabular}




\begin{tabular}{|c|c|c|c|c|c|c|}
\hline \multirow{2}{*}{ z } & \multirow{2}{*}{$\begin{array}{l}\text { Vento } \\
\text { Médio }\end{array}$} & \multicolumn{5}{|c|}{ Flutuações } \\
\hline & & Modo1 & Modo 2 & Modo 3 & Modo 4 & Modo 5 \\
\hline (m) & (kNm) & (kNm) & (kNm) & $(\mathrm{kNm})$ & (kNm) & (kNm) \\
\hline 13 & 135,51 & 284,66 & 0,06 & 4,07 & 0,30 & 0,45 \\
\hline 12 & 143,11 & 300,94 & 0,05 & 4,04 & 0,45 & 0,19 \\
\hline 11 & 150,86 & 317,36 & 0,03 & 3,83 & 0,58 & 0,10 \\
\hline 10 & 158,75 & 333,91 & 0,01 & 3,46 & 0,66 & 0,38 \\
\hline 9 & 166,76 & 350,56 & 0,00 & 2,94 & 0,69 & 0,61 \\
\hline 8 & 174,90 & 367,30 & 0,02 & 2,26 & 0,66 & 0,77 \\
\hline 7 & 183,16 & 384,12 & 0,04 & 1,43 & 0,58 & 0,82 \\
\hline 6 & 191,53 & 401,00 & 0,06 & 0,48 & 0,44 & 0,76 \\
\hline 5 & 200,00 & 417,92 & 0,08 & 0,57 & 0,25 & 0,59 \\
\hline 4 & 208,56 & 434,87 & 0,10 & 1,69 & 0,02 & 0,33 \\
\hline 3 & 217,22 & 451,84 & 0,12 & 2,87 & 0,23 & 0,01 \\
\hline 2 & 225,92 & 468,82 & 0,15 & 4,07 & 0,50 & 0,39 \\
\hline 1 & 234,65 & 485,80 & 0,17 & 5,29 & 0,78 & 0,80 \\
\hline 0 & 243,41 & 502,78 & 0,19 & 6,51 & 1,06 & 1,22 \\
\hline
\end{tabular}




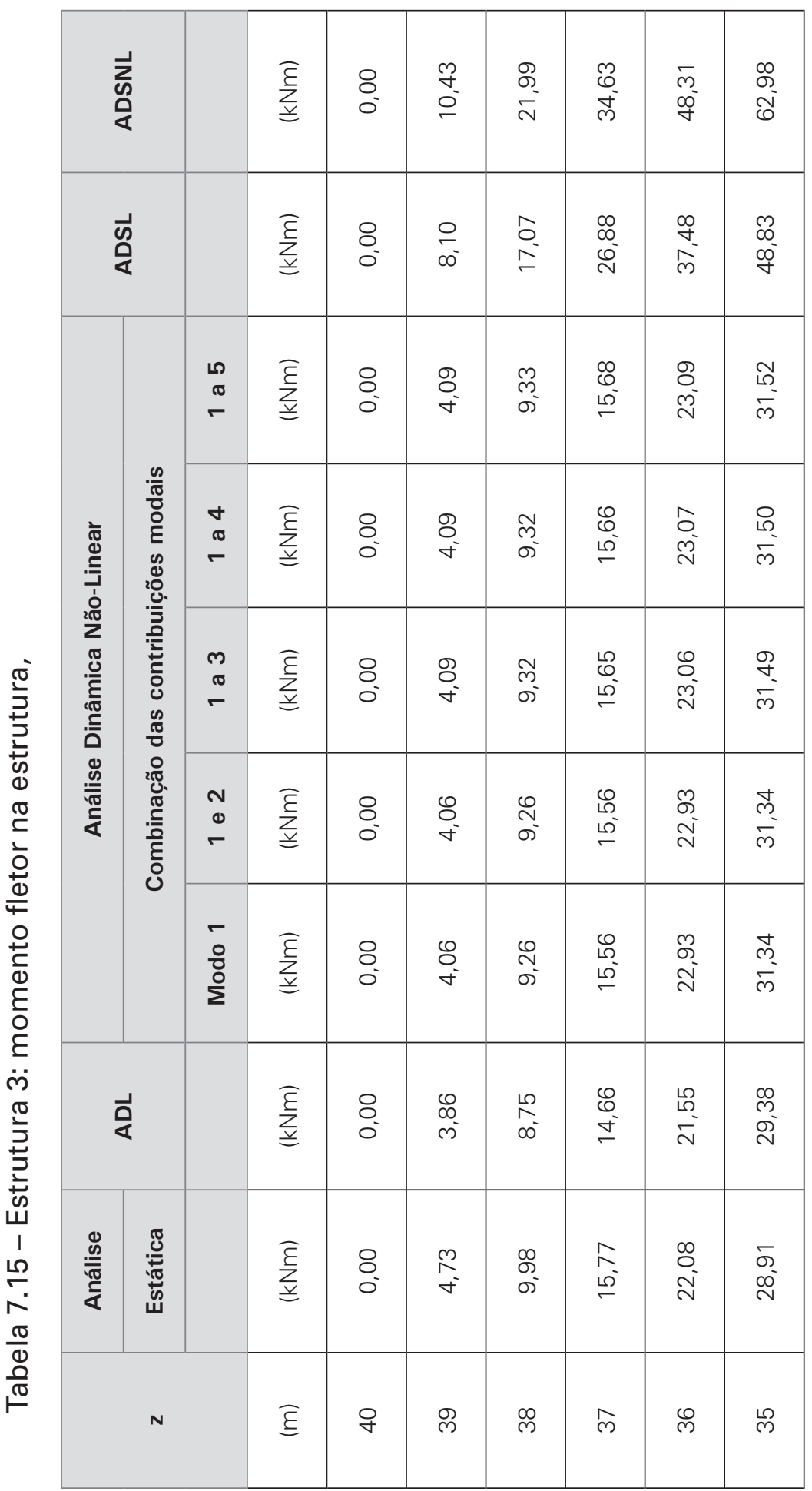




\begin{tabular}{|c|c|c|c|c|c|c|c|c|c|c|}
\hline \multicolumn{2}{|c|}{$\begin{array}{l}\text { 岕 } \\
\text { 足 }\end{array}$} & & $\begin{array}{l}8 \\
\infty \\
\infty\end{array}$ & $\frac{\sigma}{\check{\sigma}}$ & $\begin{array}{l}\text { f } \\
\stackrel{5}{\approx} \\
\approx\end{array}$ & 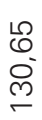 & 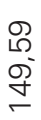 & $\begin{array}{l}\stackrel{1}{N} \\
\text { ه } \\
6\end{array}$ & $\begin{array}{l}8 \\
\bullet \\
\infty \\
\infty\end{array}$ & $\begin{array}{l}\infty \\
\stackrel{\infty}{0} \\
\stackrel{0}{N} \\
\stackrel{1}{N}\end{array}$ \\
\hline \multicolumn{2}{|c|}{ 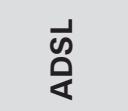 } & & $\begin{array}{l}8 \\
8 \\
8 \\
0\end{array}$ & $\begin{array}{l}\stackrel{L}{0} \\
\stackrel{0}{N}\end{array}$ & $\begin{array}{l}\text { J } \\
\text { 同 }\end{array}$ & $\begin{array}{l}\text { Ó } \\
\text { ¿ }\end{array}$ & $\begin{array}{l}8 \\
\frac{8}{\circ} \\
\leftarrow\end{array}$ & $\begin{array}{l}\bar{\pi} \\
\text { ò } \\
\text { m. }\end{array}$ & 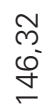 & \begin{tabular}{l} 
ㅁ \\
\multirow{N}{0}{} \\
0
\end{tabular} \\
\hline \multirow{5}{*}{ 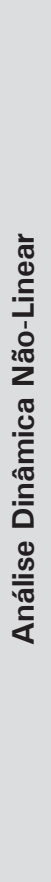 } & \multirow{5}{*}{ 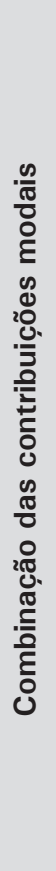 } & $\begin{array}{l}\text { م) } \\
\sigma \\
\sigma\end{array}$ & $\begin{array}{l}\text { ঠ } \\
\text { ' } \\
\text { ' }\end{array}$ & $\underset{n}{\bar{n}}$ & $\begin{array}{l}\text { ㅇ } \\
\text { ָ̃ }\end{array}$ & \begin{tabular}{r}
$\hat{N}$ \\
\multirow{N}{*}{}
\end{tabular} & $\begin{array}{l}\stackrel{\llcorner}{\infty} \\
\infty \\
\infty\end{array}$ & 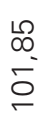 & $\begin{array}{l}\text { g } \\
6 \\
6\end{array}$ & $\begin{array}{l}\text { N } \\
\text { Nं } \\
\text { mे }\end{array}$ \\
\hline & & $\begin{array}{l}+ \\
\sigma \\
\sigma\end{array}$ & $\begin{array}{l}\text { ঠे } \\
\text { ᄋ }\end{array}$ & 으 & $\begin{array}{l}\text { న } \\
\text { ָָ } \\
\end{array}$ & $\begin{array}{l}0 \\
N \\
N\end{array}$ & $\begin{array}{l}\stackrel{\circ}{\infty} \\
\infty \\
\infty\end{array}$ & 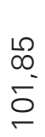 & $\begin{array}{l}8 \\
6 \\
6 \\
6\end{array}$ & $\begin{array}{l}\text { N } \\
\text { N } \\
\text { m}\end{array}$ \\
\hline & & $\begin{array}{l}\text { m } \\
\text { ర } \\
-\end{array}$ & $\begin{array}{l}\text { ঠे } \\
\text { ○ }\end{array}$ & ্ָ & 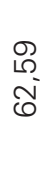 & $\begin{array}{l}\stackrel{0}{N} \\
\mathbb{N}\end{array}$ & $\begin{array}{l}\stackrel{\llcorner}{\infty} \\
\stackrel{\infty}{\infty}\end{array}$ & $\begin{array}{l}\stackrel{1}{\infty} \\
\overline{0} \\
\end{array}$ & $\begin{array}{l}8 \\
0 \\
6 \\
\sigma\end{array}$ & $\begin{array}{l}\text { N } \\
\text { N } \\
\text { m}\end{array}$ \\
\hline & & $\begin{array}{l}N \\
0 \\
\sigma\end{array}$ & \begin{tabular}{l}
\multirow{N}{*}{} \\
O্
\end{tabular} & $\begin{array}{l}\circ \\
\frac{0}{5}\end{array}$ & \begin{tabular}{l} 
ㅇ \\
\multirow{\sigma}{*}{}
\end{tabular} & $\begin{array}{l}\infty \\
\llcorner \\
\mathbb{N} \\
N\end{array}$ & $\begin{array}{l}\infty \\
0 \\
\infty \\
\infty\end{array}$ & 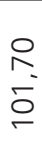 & $\begin{array}{l}\hat{L} \\
0 \\
= \\
=\end{array}$ & \begin{tabular}{l}
\multirow{N}{N}{} \\
$\underset{N}{ }$
\end{tabular} \\
\hline & & $\begin{array}{l}- \\
\text { 음 } \\
\text { 일 }\end{array}$ & \begin{tabular}{l}
\multirow{N}{*}{} \\
$\stackrel{\circ}{+}$
\end{tabular} & $\begin{array}{l}\frac{0}{5} \\
\frac{1}{5}\end{array}$ & \begin{tabular}{l} 
ㅁ \\
\multirow{\sigma}{*}{}
\end{tabular} & $\begin{array}{l}\infty \\
\llcorner \\
\mathbb{N} \\
N\end{array}$ & $\begin{array}{l}\infty \\
6 \\
\infty \\
\infty\end{array}$ & 옹 & $\begin{array}{l}\hat{L} \\
0 \\
\approx \\
\sigma\end{array}$ & \begin{tabular}{l}
\multirow{N}{N}{} \\
$\underset{N}{2}$
\end{tabular} \\
\hline \multicolumn{2}{|c|}{ 完 } & & $\frac{m}{\infty}$ & \begin{tabular}{c}
$\stackrel{0}{N}$ \\
\multirow{\sigma}{*}{}
\end{tabular} & $\begin{array}{l}\stackrel{10}{\sim} \\
\infty \\
0 \\
10\end{array}$ & 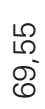 & $\begin{array}{l}\text { ) } \\
\infty \\
\infty\end{array}$ & $\begin{array}{l}\text { ఠ } \\
\text { ठ }\end{array}$ & 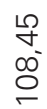 & $\begin{array}{l}\text { 吕 } \\
\text { N } \\
\text { N }\end{array}$ \\
\hline 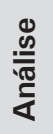 & 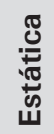 & & $\begin{array}{l}0 \\
\underset{N}{v} \\
\underset{m}{m}\end{array}$ & $\begin{array}{l}\underset{f}{f} \\
\text { f }\end{array}$ & \begin{tabular}{l} 
g \\
\multirow{N}{0}{}
\end{tabular} & 孞 & $\begin{array}{l}\stackrel{\circ}{N} \\
0^{\circ}\end{array}$ & $\begin{array}{l}\Re \\
0 \\
\infty \\
\infty\end{array}$ & $\begin{array}{l}\text { O } \\
\text { - }\end{array}$ & $\begin{array}{l}0 \\
\infty \\
5 \\
-\end{array}$ \\
\hline & $N$ & & હે & $m$ & ๗ె & $\bar{m}$ & ిల & $\stackrel{\sim}{\sim}$ & $\stackrel{\infty}{\sim}$ & $\widehat{\curvearrowright}$ \\
\hline
\end{tabular}




\begin{tabular}{|c|c|c|c|c|c|c|c|c|c|c|}
\hline \multicolumn{2}{|c|}{ 志 } & & $\begin{array}{l}\stackrel{N}{\check{N}} \\
\stackrel{\text { N }}{\mathrm{N}}\end{array}$ & $\begin{array}{l}\text { N } \\
\text { ஸे } \\
\stackrel{N}{N}\end{array}$ & 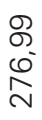 & $\begin{array}{l}\text { م) } \\
\text { ஓं }\end{array}$ & $\begin{array}{l}\stackrel{0}{N} \\
\stackrel{N}{N} \\
\stackrel{n}{n}\end{array}$ & $\frac{\stackrel{9}{N}}{\text { 官 }}$ & $\begin{array}{l}\stackrel{ }{N} \\
\underset{N}{N}\end{array}$ & 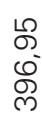 \\
\hline \multicolumn{2}{|c|}{$\begin{array}{l}\text { வ } \\
\text { Q⿱宀女 }\end{array}$} & & $\begin{array}{l}m \\
\infty \\
\infty \\
\stackrel{0}{\sim}\end{array}$ & $\begin{array}{l}\infty \\
\infty \\
\stackrel{\circ}{\sigma}\end{array}$ & $\frac{\sigma}{\frac{\sigma}{\sigma}}$ & 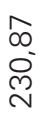 & $\begin{array}{l}\infty \\
\infty \\
\infty \\
\stackrel{+}{+}\end{array}$ & 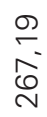 & $\frac{\infty}{\stackrel{\infty}{N}} \stackrel{\infty}{\stackrel{\infty}{N}}$ & $\begin{array}{l}\text { Ð } \\
\text { J } \\
\text { ల }\end{array}$ \\
\hline \multirow{5}{*}{ 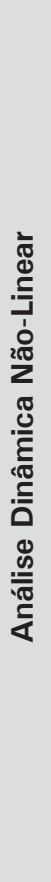 } & \multirow{5}{*}{ 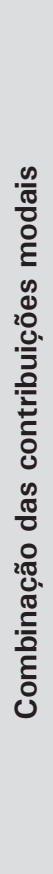 } & $\begin{array}{l}\text { L } \\
\sigma \\
\sigma\end{array}$ & $\begin{array}{l}\stackrel{0}{N} \\
\stackrel{\infty}{+} \\
\stackrel{+}{\leftarrow}\end{array}$ & $\begin{array}{l}\stackrel{\circ}{1} \\
\stackrel{0}{0} \\
\leftarrow\end{array}$ & $\begin{array}{l}\hat{L} \\
\aleph^{\circ} \\
\infty\end{array}$ & $\begin{array}{l}\text { চ } \\
\text { চ }\end{array}$ & 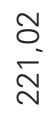 & 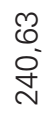 & $\begin{array}{l}\infty \\
\stackrel{\infty}{\sim} \\
\stackrel{0}{N}\end{array}$ & $\stackrel{\substack{\infty \\
\sim}}{\stackrel{\infty}{v}}$ \\
\hline & & $\begin{array}{l}+ \\
\sigma \\
\sigma\end{array}$ & $\begin{array}{l}\mathbb{8} \\
0 \\
0^{0} \\
+\end{array}$ & $\begin{array}{l}\Omega \\
0 \\
0 \\
0\end{array}$ & $\begin{array}{l}0 \\
\llcorner \\
\Gamma^{-} \\
\infty\end{array}$ & $\begin{array}{l}\text { হ } \\
\text { চ }\end{array}$ & 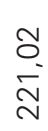 & 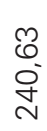 & $\begin{array}{l}\stackrel{\infty}{N} \\
\stackrel{0}{8} \\
\stackrel{0}{v}\end{array}$ & $\begin{array}{l}0 \\
\stackrel{+}{\alpha} \\
\sim \\
\sim\end{array}$ \\
\hline & & $\begin{array}{l}m \\
\sigma \\
\sigma\end{array}$ & $\begin{array}{l}\text { ऽ } \\
0 \\
\infty \\
\stackrel{+}{+}\end{array}$ & $\begin{array}{l}\text { の } \\
0 \\
0 \\
0\end{array}$ & $\begin{array}{l}0 \\
\llcorner \\
\infty \\
\infty \\
\infty\end{array}$ & 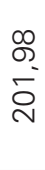 & \begin{tabular}{l}
\multirow{O}{N}{} \\
$\bar{N}$
\end{tabular} & 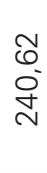 & $\begin{array}{l}\text { N } \\
\stackrel{0}{0} \\
\text { N }\end{array}$ & \begin{tabular}{l}
0 \\
\multirow{\alpha}{\alpha}{} \\
$\sim$ \\
$\sim$
\end{tabular} \\
\hline & & $\begin{array}{l}N \\
0 \\
\sigma\end{array}$ & $\begin{array}{l}\text { Ðै } \\
\infty^{-} \\
\underset{\leftarrow}{+}\end{array}$ & 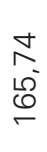 & $\begin{array}{l}\text { L } \\
\text { లో } \\
\infty\end{array}$ & $\begin{array}{l}\text { n. } \\
\text { চ্ }\end{array}$ & $\begin{array}{l}\bar{\delta} \\
\bar{N}\end{array}$ & \begin{tabular}{l}
$\widetilde{O}$ \\
0 \\
\multirow{+}{*}{}
\end{tabular} & $\begin{array}{l}\hat{N} \\
0 \\
0 \\
\mathbb{0}\end{array}$ & 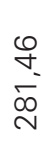 \\
\hline & & $\begin{array}{l}\text { ㅇ } \\
\frac{0}{0} \\
\Sigma\end{array}$ & 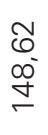 & $\frac{N}{\Delta}$ & $\begin{array}{l}\text { L } \\
\text { ஸ } \\
\infty\end{array}$ & $\begin{array}{l}\hat{\sigma} \\
\bar{\sigma} \\
\text { ¿ }\end{array}$ & $\begin{array}{l}\bar{\delta} \\
\bar{N}\end{array}$ & 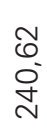 & $\begin{array}{l}\hat{N} \\
\stackrel{0}{0} \\
\stackrel{0}{0}\end{array}$ & $\begin{array}{l}0 \\
\stackrel{+}{-} \\
\stackrel{\alpha}{\sim}\end{array}$ \\
\hline \multicolumn{2}{|c|}{ 完 } & & 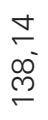 & $\begin{array}{l}\infty \\
\text { న } \\
\text { ని } \\
\end{array}$ & 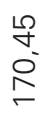 & $\begin{array}{l}\text { గN } \\
\stackrel{\infty}{\infty}\end{array}$ & 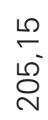 & $\begin{array}{l}\bar{m} \\
\stackrel{N}{N}\end{array}$ & 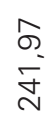 & 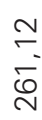 \\
\hline 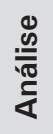 & 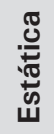 & & $\begin{array}{l}\overline{\check{n}} \\
\stackrel{\tilde{m}}{\mp}\end{array}$ & 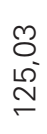 & $\stackrel{m}{m}$ & $\begin{array}{l}\check{0} \\
\circ \\
0\end{array}$ & 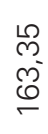 & 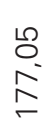 & 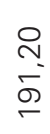 & 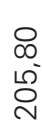 \\
\hline & $N$ & & $\stackrel{\sim}{\sim}$ & $\stackrel{\llcorner}{\sim}$ & $\stackrel{\sim}{\sim}$ & $\stackrel{N}{N}$ & $\underset{N}{ }$ & $\bar{\sim}$ & ㄱ & $\stackrel{\sigma}{\sim}$ \\
\hline
\end{tabular}




\begin{tabular}{|c|c|c|c|c|c|c|c|c|c|c|}
\hline \multicolumn{2}{|c|}{$\begin{array}{l}\text { 主 } \\
\text { 足 }\end{array}$} & & 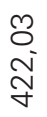 & 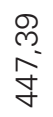 & $\begin{array}{c}\bar{\sigma} \\
\stackrel{丶}{\sigma}\end{array}$ & $\begin{array}{l}\text { م } \\
\infty \\
\infty \\
\text { م }\end{array}$ & 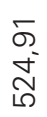 & 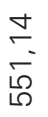 & 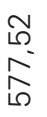 & $\begin{array}{l}\text { ঠ } \\
\text { 寸 } \\
\text { ठ }\end{array}$ \\
\hline \multicolumn{2}{|c|}{ 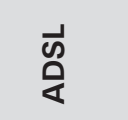 } & & $\begin{array}{l}\text { 尺 } \\
\text { Nે } \\
\text { ले }\end{array}$ & 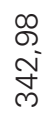 & \begin{tabular}{l} 
م \\
\multirow{2}{*}{} \\
రె
\end{tabular} & $\begin{array}{l}\text { D } \\
\text { o } \\
\text { N } \\
\text { లn }\end{array}$ & $\begin{array}{l}\infty \\
\infty \\
-5 \\
\dot{\gamma}\end{array}$ & $\begin{array}{l}\bar{\infty} \\
\underset{\sim}{\sim}\end{array}$ & 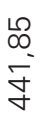 & $\begin{array}{l}\text { S } \\
\bar{\sigma} \\
\dot{\sigma}\end{array}$ \\
\hline \multirow{5}{*}{ 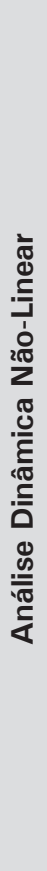 } & \multirow{5}{*}{ 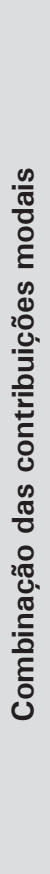 } & $\begin{array}{l}\text { م) } \\
\text { ס }\end{array}$ & $\begin{array}{l}\infty \\
\mathbb{0} \\
\text { Nं } \\
\text { ঠे }\end{array}$ & $\begin{array}{l}\bar{J} \\
\underset{J}{J} \\
m\end{array}$ & $\begin{array}{l}0 \\
0 \\
0 \\
\text { மे }\end{array}$ & 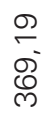 & 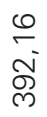 & 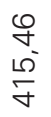 & 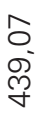 & 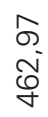 \\
\hline & & $\begin{array}{l}+ \\
\text { ர } \\
\sigma\end{array}$ & 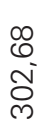 & $\begin{array}{l}\underset{J}{J} \\
\underset{\sim}{J}\end{array}$ & $\begin{array}{l}8 \\
8 \\
0 \\
\text { ల్ }\end{array}$ & 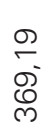 & $\begin{array}{l}\frac{0}{2} \\
\text { ळे }\end{array}$ & 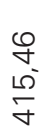 & $\begin{array}{l}\text { ô } \\
\text { गे } \\
\text { ণ }\end{array}$ & $\begin{array}{l}\hat{\sigma} \\
\text { ô } \\
0\end{array}$ \\
\hline & & $\begin{array}{l}m \\
\text { ס } \\
\sigma\end{array}$ & 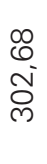 & $\begin{array}{l}\bar{J} \\
\underset{J}{J} \\
\text { J }\end{array}$ & $\begin{array}{l}0 \\
8 \\
0 \\
\text { ల) }\end{array}$ & 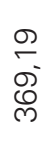 & 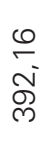 & $\begin{array}{l}\dot{O} \\
\stackrel{\sigma}{\circ} \\
\frac{\sigma}{+}\end{array}$ & $\begin{array}{l}\text { ô } \\
\text { गे } \\
\text { ॄे }\end{array}$ & 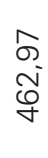 \\
\hline & & $\begin{array}{l}N \\
0 \\
\sigma\end{array}$ & $\begin{array}{l}\hat{0} \\
\text { N } \\
\text { Oे }\end{array}$ & $\begin{array}{l}\stackrel{+}{+} \\
\stackrel{ \pm}{N} \\
\text { D }\end{array}$ & $\begin{array}{l}\infty \\
\llcorner \\
0^{\circ} \\
\stackrel{0}{m}\end{array}$ & $\begin{array}{l}\text { E- } \\
\text { s. } \\
0 \\
\text { ल }\end{array}$ & $\begin{array}{l}\frac{m}{5} \\
\text { مे }\end{array}$ & $\begin{array}{l}\frac{M}{+} \\
\frac{1}{+}\end{array}$ & 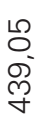 & 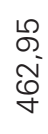 \\
\hline & & $\begin{array}{l}- \\
\text { 음 } \\
\text { 몬 }\end{array}$ & $\begin{array}{l}\hat{\theta} \\
\text { ঠे } \\
\text { లి }\end{array}$ & $\begin{array}{l}\stackrel{P}{+} \\
\stackrel{d}{\sim} \\
\text { }\end{array}$ & $\begin{array}{l}\infty \\
\llcorner \\
0^{\circ} \\
\text { లે }\end{array}$ & 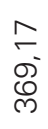 & $\frac{\text { m}}{\text { ণ }}$ & $\begin{array}{l}\stackrel{M}{+} \\
\frac{1}{\sigma}\end{array}$ & 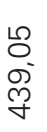 & 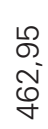 \\
\hline \multicolumn{2}{|c|}{ 完 } & & $\begin{array}{l}\hat{N} \\
\stackrel{\circ}{\circ} \\
\stackrel{0}{N}\end{array}$ & $\begin{array}{l}8 \\
8 \\
8 \\
\text { ○े }\end{array}$ & 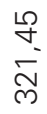 & 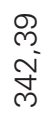 & $\begin{array}{l}\hat{0} \\
\text { లె } \\
\text { లె }\end{array}$ & $\begin{array}{l}\infty \\
\stackrel{\infty}{N} \\
\stackrel{\sim}{\infty} \\
\infty \\
\sim\end{array}$ & 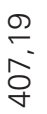 & 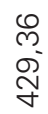 \\
\hline 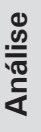 & 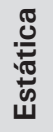 & & 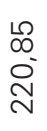 & $\begin{array}{l}\text { లె } \\
0^{-} \\
\text {N }\end{array}$ & $\begin{array}{l}0 \\
\stackrel{N}{N} \\
\stackrel{N}{N}\end{array}$ & $\begin{array}{l}\circ \\
\varnothing \\
\infty \\
0 \\
\sim\end{array}$ & $\begin{array}{l}\hat{m} \\
\omega^{\circ} \\
\stackrel{N}{N}\end{array}$ & 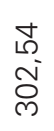 & $\begin{array}{l}\text { N } \\
\text { సे } \\
\text { m) }\end{array}$ & $\begin{array}{l}\text { व. } \\
0 \\
m \\
m\end{array}$ \\
\hline & $\mathbf{N}$ & & $\stackrel{\infty}{-}$ & $\approx$ & $\stackrel{\bullet}{\leftarrow}$ & $\stackrel{\sim}{\leftarrow}$ & 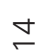 & $\stackrel{m}{\leftarrow}$ & $\simeq$ & $\approx$ \\
\hline
\end{tabular}




\begin{tabular}{|c|c|c|c|c|c|c|c|c|c|c|}
\hline \multicolumn{2}{|c|}{$\begin{array}{l}\text { 之. } \\
\text { 岁 } \\
\text { Q }\end{array}$} & & 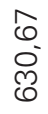 & 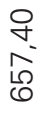 & \begin{tabular}{l}
$\stackrel{\bigcirc}{N}$ \\
\multirow{D}{*}{} \\
0
\end{tabular} & 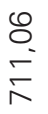 & مิ & 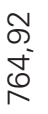 & 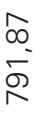 & $\begin{array}{l}m \\
\infty \\
\infty \\
\infty\end{array}$ \\
\hline \multicolumn{2}{|c|}{$\begin{array}{l}\text { 㟔 } \\
\text { 峞 }\end{array}$} & & 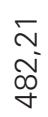 & 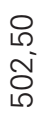 & 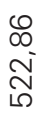 & 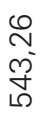 & $\begin{array}{l}\stackrel{0}{1} \\
\text { ల̃ } \\
0\end{array}$ & 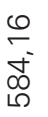 & 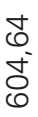 & 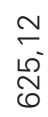 \\
\hline \multirow{5}{*}{ 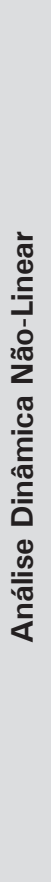 } & \multirow{5}{*}{ 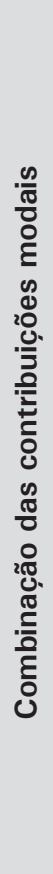 } & $\begin{array}{l}\text { م) } \\
\sigma \\
\sigma\end{array}$ & 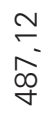 & $\frac{\circ}{\check{\leftarrow}}$ & 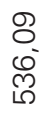 & $\begin{array}{l}\infty \\
\infty \\
\varnothing \\
\varnothing \\
\varnothing \\
\llcorner\end{array}$ & $\begin{array}{l}\circlearrowleft \\
\infty \\
\llcorner \\
\infty \\
\infty \\
\llcorner\end{array}$ & $\begin{array}{l}\text { ঠे } \\
0 \\
\frac{0}{6}\end{array}$ & $\begin{array}{l}0 \\
\qquad 0^{\circ} \\
\tilde{\omega}\end{array}$ & 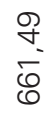 \\
\hline & & $\begin{array}{l}+ \\
\sigma \\
\sigma\end{array}$ & 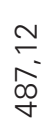 & $\frac{O}{\stackrel{0}{\circ}}$ & $\begin{array}{l}8 \\
\circ \\
0 \\
\tilde{N} \\
\llcorner\end{array}$ & $\begin{array}{l}\infty \\
\infty \\
\infty \\
\varnothing \\
\varnothing \\
\llcorner \\
\llcorner\end{array}$ & $\begin{array}{l}M \\
\infty \\
\infty \\
\infty \\
\infty \\
\llcorner\end{array}$ & 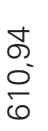 & 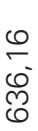 & $\begin{array}{l}\text { P. } \\
\overline{0} \\
\overline{0}\end{array}$ \\
\hline & & $\begin{array}{l}m \\
\widetilde{\sigma} \\
-\end{array}$ & 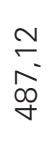 & 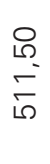 & $\begin{array}{l}8 \\
0 \\
0 \\
\tilde{1} \\
\llcorner\end{array}$ & 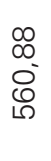 & $\begin{array}{l}m \\
\infty \\
\infty \\
\infty \\
\infty\end{array}$ & 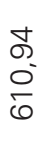 & $\begin{array}{l}0 \\
\overline{0} \\
\tilde{ల} \\
\tilde{c}\end{array}$ & $\begin{array}{l}\text { g. } \\
\overline{8} \\
\overline{8}\end{array}$ \\
\hline & & $\begin{array}{l}N \\
0 \\
\sigma\end{array}$ & 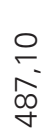 & 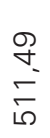 & 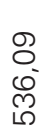 & $\begin{array}{l}\infty \\
\infty \\
\infty \\
\infty \\
\varnothing \\
\infty\end{array}$ & $\begin{array}{l}m \\
\infty \\
\infty \\
\infty \\
\infty\end{array}$ & $\begin{array}{l}\text { ठ } \\
\text { ○' } \\
\overline{6}\end{array}$ & 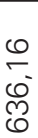 & $\begin{array}{l}\stackrel{\infty}{+} \\
\underset{\theta}{0}\end{array}$ \\
\hline & & $\begin{array}{l}\overline{-} \\
\frac{0}{0} \\
\Sigma\end{array}$ & 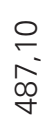 & $\frac{\text { q }}{\check{\leftarrow}}$ & $\begin{array}{l}9 \\
0 \\
0 \\
\text { లె }\end{array}$ & $\begin{array}{l}\infty \\
\infty \\
\infty \\
\circ \\
\bullet \\
\llcorner\end{array}$ & $\begin{array}{l}M \\
\infty \\
\infty \\
\infty \\
\infty \\
\infty\end{array}$ & $\begin{array}{l}\text { ठ } \\
\text { ○ } \\
\bar{\sigma}\end{array}$ & $\begin{array}{l}0 \\
\qquad 0^{\circ} \\
\widetilde{0}\end{array}$ & $\begin{array}{l}\infty \\
\stackrel{\infty}{+} \\
\overline{8} \\
\mathbb{8}\end{array}$ \\
\hline \multicolumn{2}{|c|}{ 完 } & & $\frac{\infty}{\stackrel{\Sigma}{\sigma}}$ & 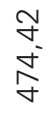 & 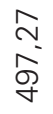 & 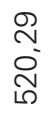 & $\begin{array}{l}\stackrel{\infty}{+} \\
\text { ஸ் } \\
\leftarrow\end{array}$ & $\begin{array}{l}\Upsilon \\
\infty \\
0 \\
0 \\
\mathbb{0} \\
10\end{array}$ & 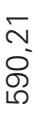 & $\begin{array}{l}0 \\
0 \\
\text { ల் } \\
\sigma\end{array}$ \\
\hline 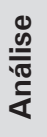 & 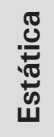 & & 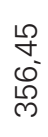 & $\frac{\sigma}{\stackrel{\sigma}{\infty}}$ & $\begin{array}{l}\text { ০ } \\
\text { ळे } \\
\text { ळे }\end{array}$ & $\begin{array}{l}\hat{N} \\
\frac{j}{\sigma}\end{array}$ & $\begin{array}{l}\infty \\
\stackrel{\infty}{\rho} \\
\text { ஸ் } \\
\text { యे }\end{array}$ & 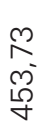 & $\frac{\sigma}{\underset{J}{J}}$ & \begin{tabular}{l}
$\stackrel{\text { }}{\Omega}$ \\
\multirow{+}{\sigma}{}
\end{tabular} \\
\hline & $N$ & & 으 & $\sigma$ & $\infty$ & 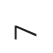 & 0 & م & $\checkmark$ & $m$ \\
\hline
\end{tabular}




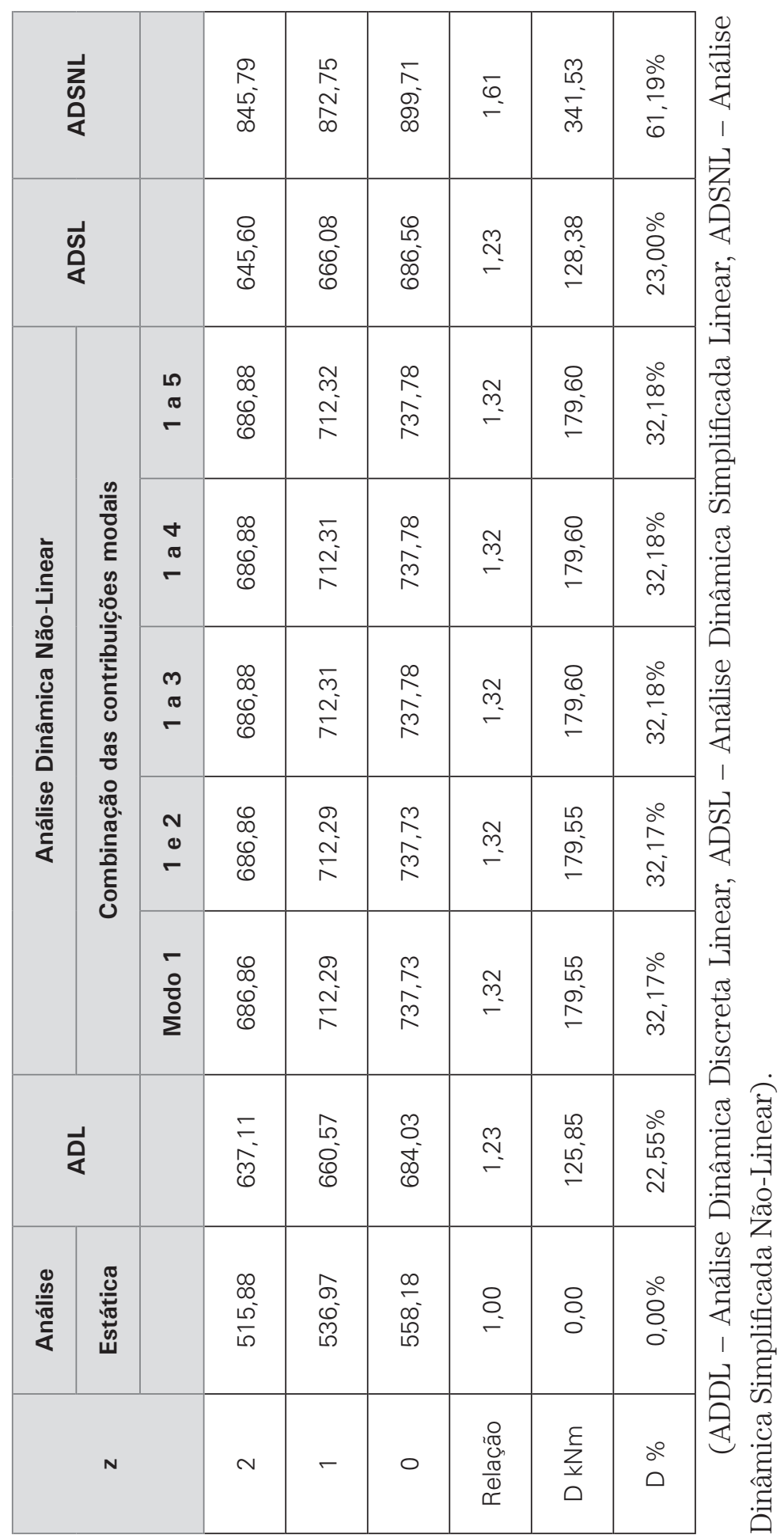


No gráfico da Figura 7.17 podem ser encontrados os resultados obtidos para a ação do vento sobre a estrutura. Nele se encontram, de baixo para cima, e nessa ordem, a análise estática, as análises dinâmicas discretas intercaladas pela análise dinâmica simplificada linear e, por último, a análise dinâmica simplificada não-linear.

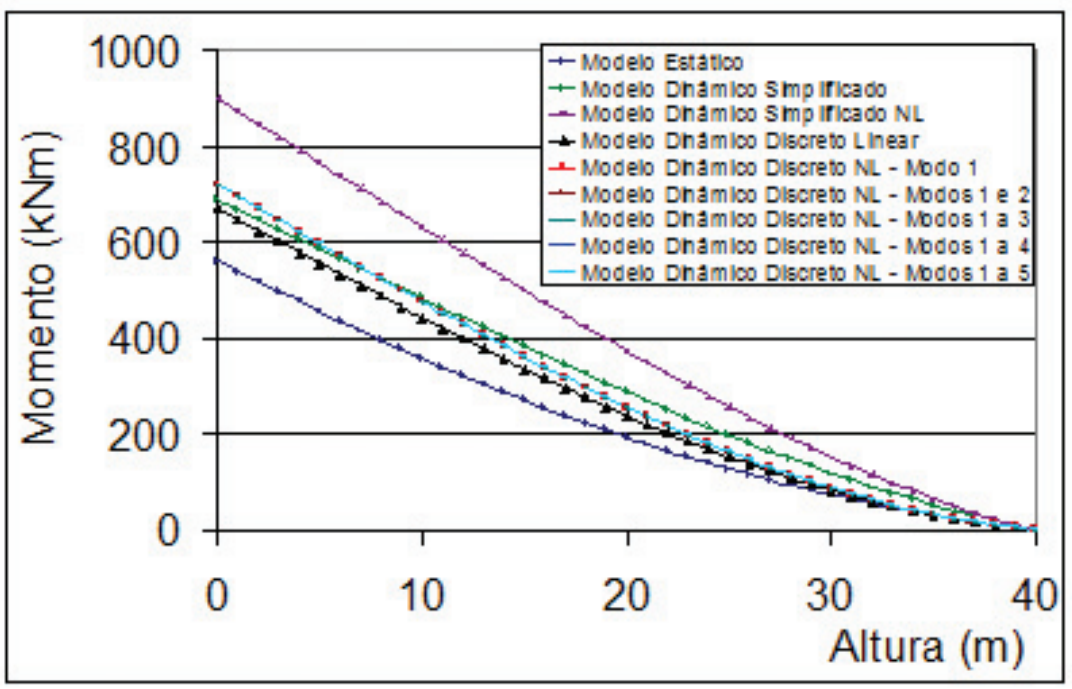

Figura 7.17 - Ação do vento na estrutura 3.

As formas modais da NBR 6123/88, a do Método dos Elementos Finitos e do método proposto constam na Figura 7.18 (a).

Uma melhor aproximação à forma modal não-linear é conseguida substituindo-se o expoente da expressão (6.6) por 1,60; tendo em vista que a curva definida com esse novo expoente oferece uma menor diferença entre seus pontos e os pontos da curva da forma modal não-linear, como pode ser observado na Figura 7.18(b).

O expoente sugerido anteriormente situa-se $6 \%$ abaixo do valor recomendado pela NBR 6123/88. 


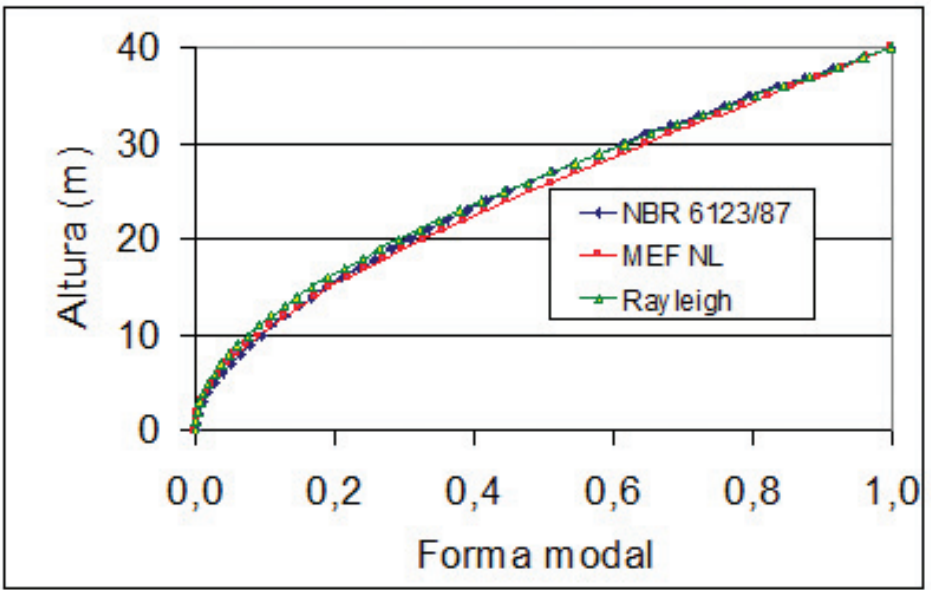

(a) expoente da forma modal: $\gamma=1,7$

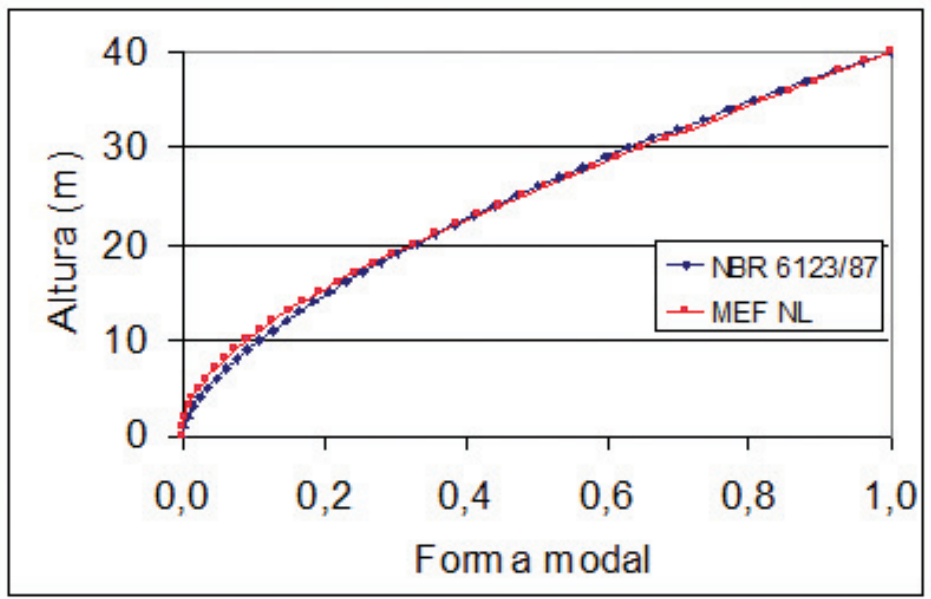

(b) expoente da forma moda: $\gamma=1,60$

Figura 7.18 - Estrutura 3 - comparativo das formas modais: (a) usada na análise, (b) sugerida. 


\subsection{ESTRUTURA 4 - POSTE DE CONCRETO ARMADO DE $46 \mathrm{M}$}

\subsubsection{Dados e geometria}

Esta é mais uma estrutura destinada ao serviço de telecomunicações. Trata-se de um poste de concreto armado com 46 metros de comprimento, incluindo a fundação e com $40 \mathrm{~m}$ de altura fora do solo. O poste possui seção circular vazada, com trechos retos e trechos que variam com a altura. A sua esbeltez, incluindo a fundação, é de 334, sem a fundação passa a 375. O módulo de elasticidade do concreto foi calculado com $\mathrm{f}_{\mathrm{ck}}$ de $45 \mathrm{MPa}$, para o poste, e de $20 \mathrm{MPa}$ para a fundação, conforme os dados recebidos e como é usual na construção dessas estruturas. Verificou-se que a condição do limite de esbeltez requerida pelo item 15.8.1 da NBR 6118/04 foi atendida.

Para poder avaliar as diferenças produzidas entre uma análise puramente linear, tanto do ponto de vista material quanto geométrico, e outra análise completamente não-linear, também em ambos os aspectos, foi adicionada à análise não-linear geométrica a não-linearidade material do concreto.

Esse tipo de estrutura é predominantemente submetida à flexão, cujo coeficiente Gama Z $\left(\gamma_{\mathrm{z}}\right)$, obtido pela análise dinâmica discreta linear, para esse caso, é 1,05. O coeficiente Gama Z está previsto no item 15.5.3 da NBR 6118/04 - Projeto de estruturas de concreto e fornece uma medida da importância dos esforços de segunda ordem globais, sendo calculado pela seguinte expressão:

$$
\gamma_{\mathrm{z}}=\frac{1}{1-\frac{\Delta \mathrm{M}_{\mathrm{tot}, \mathrm{d}}}{\mathrm{M}_{1, \mathrm{tot}, \mathrm{d}}}},
$$

onde: 
$\mathrm{M}_{1, \text { tot,d }}$ é o momento de tombamento em relação à base da estrutura,

$\Delta \mathrm{M}_{\text {tot,d }}$ é a soma dos produtos de todas as forças verticais atuantes na estrutura pelos deslocamentos de seus respectivos pontos de aplicação, obtidos na análise de $1^{\text {a }}$ ordem.

O item 15.7.3 da NBR 6118/03, permite usar 0,5 $\mathrm{E}_{\mathrm{ci}} \mathrm{I}_{\mathrm{c}}$ nas análises aproximadas para a consideração da não-linearidade física do concreto, para estruturas com Gama Z inferior a 1,3.

Brasil, Silva e Wahrhaftig (2007); e Silva \& Brasil (2006), ao estudarem a estrutura da seção anterior, verificaram, por meio do conceito do produto de rigidez efetiva, que a relação entre a inércia efetivamente solicitada e a inércia total da seção de concreto armado é de 0,4 , quando o momento fletor atuante se iguala ao momento último resistente da seção.

A rotação calculada para o correspondente deslocamento horizontal, no cálculo do coeficiente Gama Z, foi de 0,045 rd, o que permite enquadrar a cinemática da estrutura na hipótese de rotações muito pequenas, conforme as definições de Souza Lima e Venâncio Filho (1982).

Ressalta-se que a inércia da seção deve ser majorada em função da seção de aço existente, como visto no item anterior, que deixa de ser aplicada por falta de informação a respeito.

Na estrutura há um conjunto de antenas e uma plataforma fixada no topo que totalizam a massa de 1097,76 kg. Há ainda escada e guarda-corpo que conferem à estrutura uma massa adicional distribuída de $40 \mathrm{~kg} / \mathrm{m}$. As áreas de exposição ao vento computadas foram de 9,34 m²; 1,40 m²; $0,40 \mathrm{~m}^{2} / \mathrm{m}$ e $0,05 \mathrm{~m}^{2} / \mathrm{m}$; respectivamente para as antenas; plataforma; escada, guarda-corpo, cabos e esteiramento. Os coeficientes de arrasto utilizados foram: 0,6 para o poste; escada, cabos e esteiramento 1,2; plataforma 2 e antenas 1.

O solo lateral foi representado por molas distribuídas de rigidez igual a $2668,93 \mathrm{kN} / \mathrm{m}^{3}\left(300 \mathrm{tf} / \mathrm{m}^{3}\right)$. A massa específica adotada para o concreto armado da fundação foi de $2500 \mathrm{~kg} / \mathrm{m}^{3}$, enquanto que o da superestrutura foi considerado de $2600 \mathrm{~kg} / \mathrm{m}^{3}$, por ser concreto centrifugado.

Os dados da geometria da superestrutura constam na Tabela 7.16. A fundação é do tipo tubulão com as seguintes características: diâmetro da base $140 \mathrm{~cm}$, diâmetro do fuste $80 \mathrm{~cm}$, comprimento do fuste $580 \mathrm{~cm}$ e altura da base $20 \mathrm{~cm}$, conforme disposto na Tabela 7.17. 


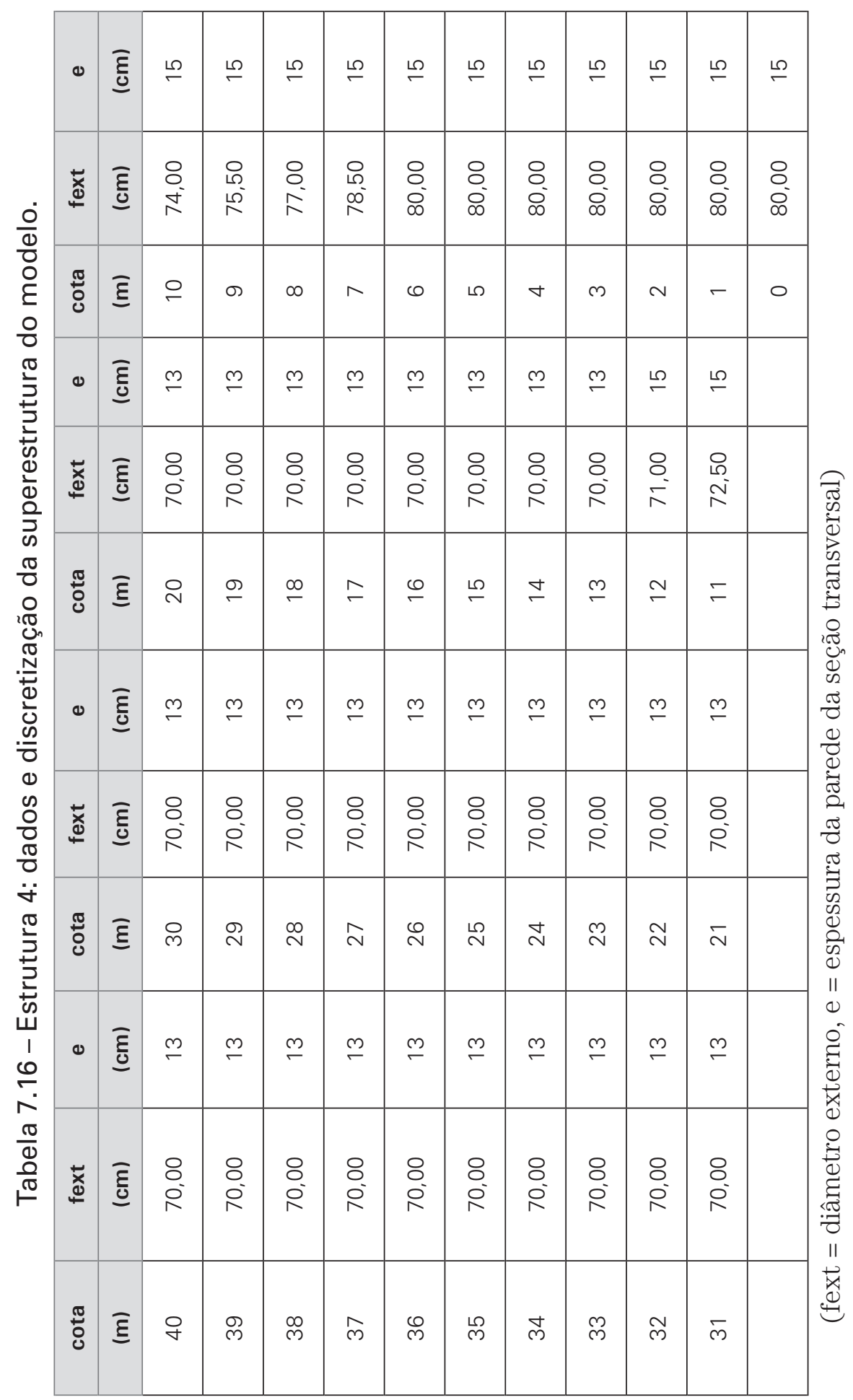


Tabela 7.17 - Estrutura 4: dados e discretização da fundação.

\begin{tabular}{|c|c|}
\hline Diâmetro da base & $140 \mathrm{~cm}$ \\
\hline Diâmetro do fuste & $80 \mathrm{~cm}$ \\
\hline Altura do fuste & $580 \mathrm{~cm}$ \\
\hline Altura da base & $20 \mathrm{~cm}$ \\
\hline
\end{tabular}

Fotografias da estrutura podem ser vistas na Figura 7.20, assim como, a geometria na Figura 7.19.
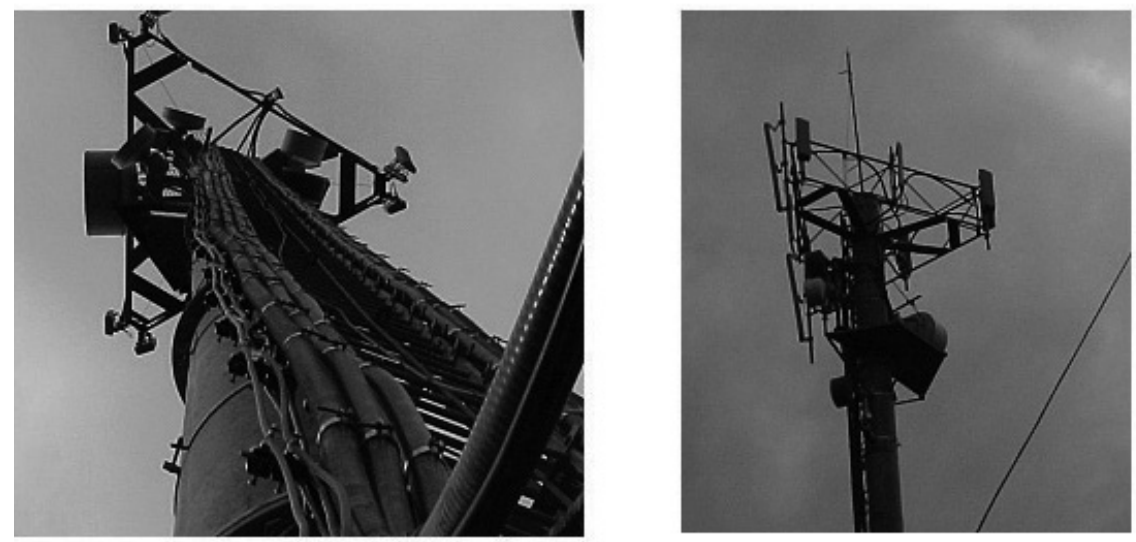

Figura 7.19 - Estrutura 4: Fotografias. 


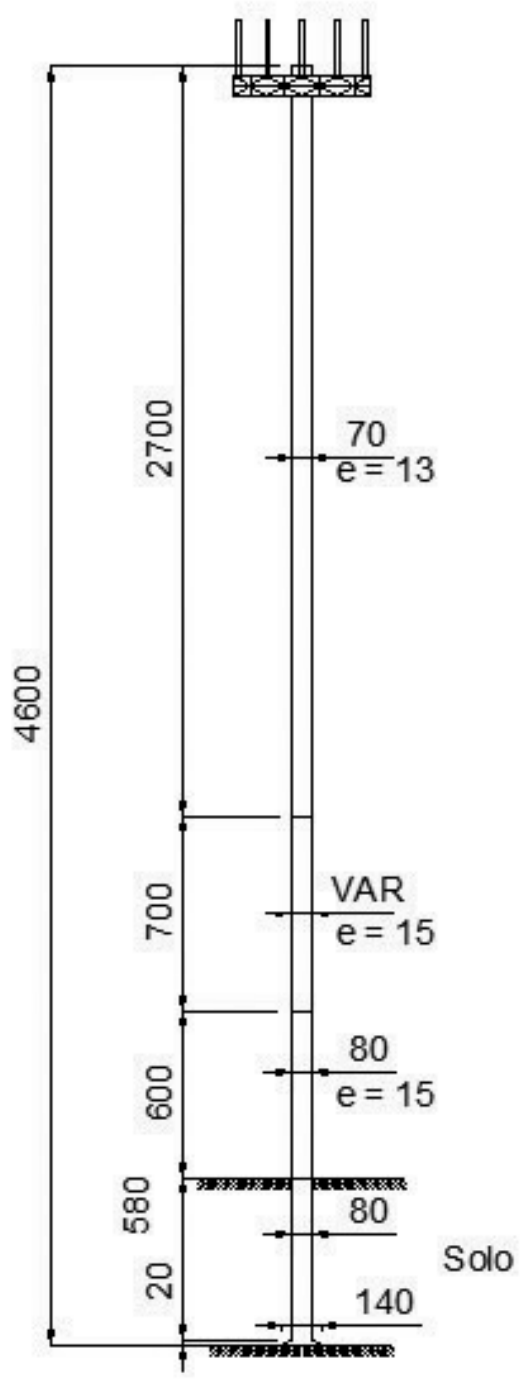

Figura 7.20 - Estrutura 4: geometria - medidas em centímetros.

\subsubsection{Modelagem por Elementos Finitos}

A estrutura foi modelada utilizando-se elementos de barra, com seções transversais constantes e variáveis. Foram feitos dois modelos. O primeiro, com $46 \mathrm{~m}$ de altura e 51 elementos, engloba, tanto as fundações quanto a superestrutura. Nesse modelo, a estrutura foi 
engastada na base, tendo o solo sido representado por molas laterais distribuídas. O segundo modelo também emprega elementos de barra de seção constante e variável, porém, a estrutura foi modelada engastando-a exclusivamente na base e com $40 \mathrm{~m}$ de altura a partir da superfície do terreno.

Em ambos os modelos foram consideradas a massa concentrada no topo e a massa distribuída devido à escada, cabos e esteiramento. Essas massas produzem os esforços normais externos atuantes na estrutura, que também foram introduzidos no modelo. Tanto a massa própria quanto o peso próprio da estrutura, foram calculadas automaticamente pelo programa SAP2000.

A Figura 7.21 e a Figura 7.23 trazem os modelos com a discretização da estrutura. Os modos de vibração e as frequências obtidas pelo Método dos Elementos Finitos estão mostradas na Figura 7.22 e Figura 7.24.

- $\quad$ Modelo com $46 \mathrm{~m}$ de altura.

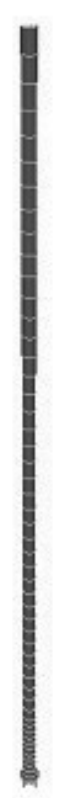

(a) $3 \mathrm{D}$

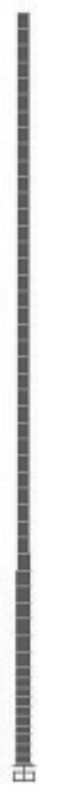

(b) Vista lateral

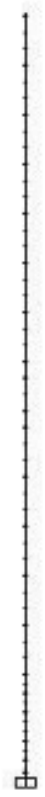

(a) Discretização

Figura 7.21 - Estrutura 4: Modelo de $46 \mathrm{~m}$ por Elementos Finitos. 


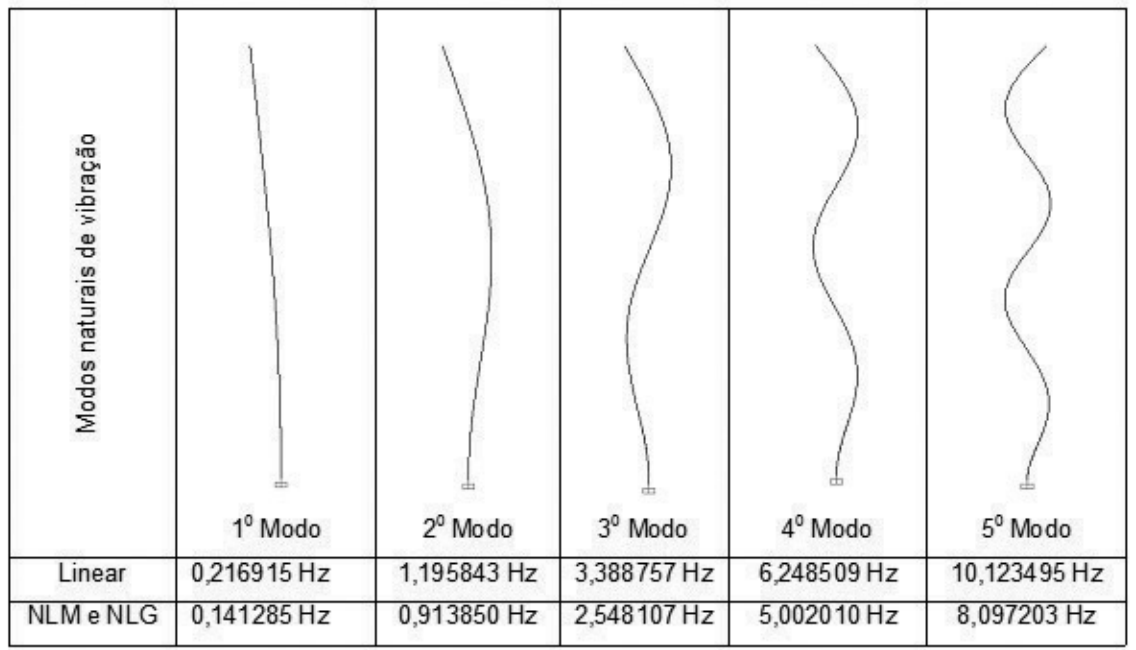

(NLG diz respeito à não-linearidade geométrica e NLM à não-linearidade material)

Figura 7.22 - Estrutura 4: modelo de $46 \mathrm{~m}$ - modos naturais de vibração.

- $\quad$ Modelo com $40 \mathrm{~m}$ de altura.

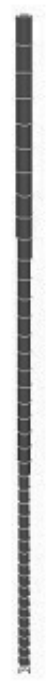

(a) $3 \mathrm{D}$

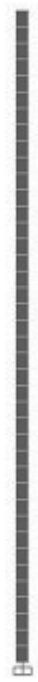

(a) Vista lateral

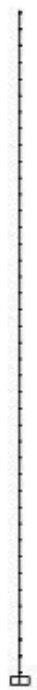

(a) Discretização

Figura 7.23 - Estrutura 4: modelo com 40 m por Elementos Finitos. 


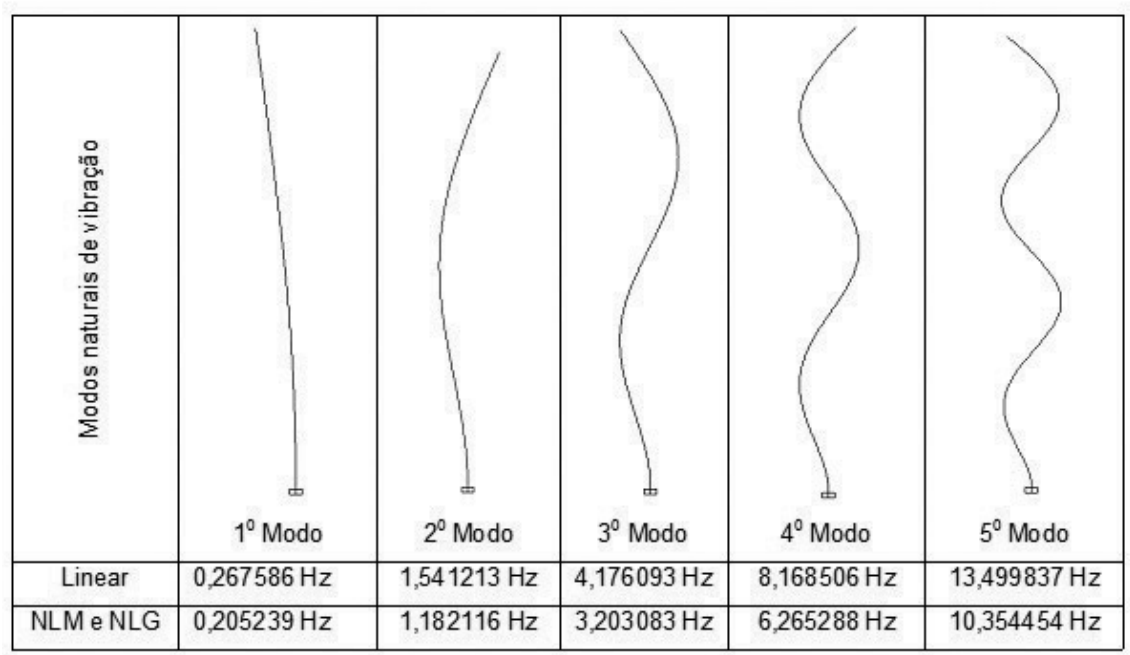

(NLG diz respeito à não-linearidade geométrica e NLM à não-linearidade material)

Figura 7.24 - Estrutura 4: modelo de 40 m - modos naturais de vibração.

\subsubsection{Aplicação do método proposto}

\subsubsection{Definição dos parâmetros}

Para esta estrutura devem ser observados: na fundação trechos de comprimento 0,2 m e 5,80 m, diâmetros de $140 \mathrm{~cm}, 80 \mathrm{~cm}$ e um trecho de diâmetro variável, módulo de elasticidade $\mathrm{E}_{1}$ de $12522 \mathrm{MPa}$ e densidade $\rho_{1}$ de $2500 \mathrm{~kg} / \mathrm{m}^{3}$. Para a superestrutura trechos de comprimento de $6 \mathrm{~m}, 7 \mathrm{~m}$ e $27 \mathrm{~m}$, seção circular vazada com espessuras de $15 \mathrm{~cm}$ e $13 \mathrm{~cm}$; diâmetros externos de $80 \mathrm{~cm}, 70 \mathrm{~cm}$ e um trecho variável, módulo de elasticidade $\mathrm{E}$ de $18783 \mathrm{MPa}$ e densidade do concreto $\rho$ de $2600 \mathrm{~kg} / \mathrm{m}^{3}$. Os módulos de elasticidade do concreto, incluindo-se a não-linearidade material, passam a ser $14901 \mathrm{MPa}$ e $22352 \mathrm{MPa}$, respectivamente para a fundação e para a superestrutura. 
Conforme descrito no item 7.4.1, a estrutura ainda suporta uma massa concentrada na extremidade superior, uma massa distribuída ao longo do seu comprimento e sua fundação está sujeita ação lateral do solo.

Ficam definidas as seguintes ordenas referentes às alturas na estrutura: $\mathrm{L}_{1}=0,2 \mathrm{~m}, \mathrm{~L}_{2}=6,0 \mathrm{~m}, \mathrm{~L}_{3}=12,0 \mathrm{~m}, \mathrm{~L}_{4}=19,0 \mathrm{~m}$, $\mathrm{L}_{5}=46,0 \mathrm{~m}$. Na base da fundação tem-se: $\mathrm{D}_{1}=140 \mathrm{~cm}, \mathrm{~A}_{1}=\frac{\pi}{4} \mathrm{D}_{1}{ }^{2}$, $\mathrm{I}_{1}=\frac{\pi}{64} \mathrm{D}_{1}{ }^{4}$. No fuste $\mathrm{D}_{2}=80 \mathrm{~cm}, \mathrm{~A}_{2}=\frac{\pi}{4} \mathrm{D}_{2}{ }^{2}, \mathrm{I}_{2}=\frac{\pi}{64} \mathrm{D}_{2}{ }^{4}$. O diâmetro do trecho variável entre a base e o fuste será obtido por interpolação linear fazendo-se $D_{1}(x)=\frac{D_{2}-D_{1}}{L_{1}} x+D_{1}$. Assim, a área e a inércia da seção serão dadas por $\mathrm{A}_{1}(\mathrm{x})=\frac{\pi}{4} \mathrm{D} 1(\mathrm{x})^{2}$ e $\mathrm{I}_{1}(\mathrm{x})=\frac{\pi}{64} \mathrm{D}_{1}(\mathrm{x})^{4}$.

Chamando de $\mathrm{D}_{3}$ e $\mathrm{e}_{3}$ o diâmetro externo e a espessura da seção inicial da superestrutura, tem-se: $D_{3}=80 \mathrm{~cm}$ e $e_{3}=13 \mathrm{~cm}$. Logo, o diâmetro interno, a área e a inércia dessa seção são: $d_{3}=D_{3}-2 e_{3}$, $\mathrm{A}_{3}=\frac{\pi}{4}\left(\mathrm{D}_{3}{ }^{2}-\mathrm{d}_{3}{ }^{2}\right)$ e $\mathrm{I}_{3}=\frac{\pi}{64}\left(\mathrm{D}_{3}{ }^{4}-\mathrm{d}_{3}{ }^{4}\right)$. Analogamente, $\mathrm{D}_{5}, \mathrm{e}_{5}$, $\mathrm{d}_{5}, \mathrm{~A}_{5}$ e $\mathrm{I}_{5}$ são o diâmetro externo, a espessura, a área e a inércia referente ao último segmento. Entre esses dois trechos há um de seção variável, de espessura igual à do segmento anterior $\left(\mathrm{e}_{4}=\mathrm{e}_{3}\right)$, cujo diâmetro pode ser obtido por interpolação linear de maneira semelhante ao que foi feito na primeira variação, usando a expressão $\mathrm{D}_{4}(\mathrm{x})=\frac{\mathrm{D}_{4}-\mathrm{D}_{3}}{\mathrm{~L}_{4}-\mathrm{L}_{3}}\left(\mathrm{x}-\mathrm{L}_{3}\right)+\mathrm{D}_{3}$. Portanto, $\mathrm{d}_{4}(\mathrm{x})=\mathrm{D}_{4}(\mathrm{x})-2 \mathrm{e}_{4}$, $\mathrm{A}_{4}(\mathrm{x})=\frac{\pi}{64}\left(\mathrm{D}_{4}(\mathrm{x})^{4}-\mathrm{d}_{4}(\mathrm{x})^{4}\right)$ e $\mathrm{I}_{4}(\mathrm{x})=\frac{\pi}{64}\left(\mathrm{D}_{4}(\mathrm{x})^{4}-\mathrm{d}_{4}(\mathrm{x})^{4}\right)$ são, respectivamente, o diâmetro interno, a área e a inércia variável da seção do trecho correspondente. 


\subsubsection{Cálculo da massa generalizada}

A massa generalizada foi obtida por meio das seguintes integrais:

$$
\begin{aligned}
& \mathrm{m}_{1}=\int_{0}^{\mathrm{L}_{1}} \mathrm{~m}_{\mathrm{I}}(\mathrm{x}) \phi(\mathrm{x})^{2} \mathrm{dx}, \text { com } \mathrm{m}_{\mathrm{I}}(\mathrm{x})=\left(\frac{\mathrm{A}_{2}-\mathrm{A}_{1}}{\mathrm{~L}_{1}}+\mathrm{A}_{1}\right) \rho_{1} ; \\
& \mathrm{m}_{2}=\int_{\mathrm{L}_{1}}^{\mathrm{L}_{2}} \mathrm{~m}_{\mathrm{II}} \phi(\mathrm{x})^{2} \mathrm{dx}, \text { com } \mathrm{m}_{\mathrm{II}}=\mathrm{A}_{2} \rho_{1} ; \\
& \mathrm{m}_{3}=\int_{\mathrm{L}_{2}}^{\mathrm{L}_{3}} \mathrm{~m}_{\mathrm{III}} \phi(\mathrm{x})^{2} \mathrm{dx}, \text { com } \mathrm{m}_{\mathrm{III}}=\mathrm{A}_{3} \rho+\mathrm{m}_{\mathrm{e}} ; \\
& \mathrm{m}_{4}=\int_{\mathrm{L} 3}^{\mathrm{L}_{4}} \mathrm{~m}_{\mathrm{IV}}(\mathrm{x}) \phi(\mathrm{x})^{2} \mathrm{dx}, \text { com } \mathrm{m}_{\mathrm{IV}}=\mathrm{A}_{4}(\mathrm{x}) \rho+\mathrm{m}_{\mathrm{e}} \mathrm{e} \\
& \mathrm{A}_{4}(\mathrm{x})=\frac{\pi}{4}\left(\mathrm{D}_{4}(\mathrm{x})^{2}-\mathrm{d}_{4}(\mathrm{x})^{2}\right) ; \\
& \mathrm{e} \\
& \mathrm{m}_{5}=\int_{\mathrm{L}_{4}}^{\mathrm{L}} \mathrm{m}_{\mathrm{V}} \phi(\mathrm{x})^{2} \mathrm{dx}, \text { com } \mathrm{m}_{\mathrm{V}}=\mathrm{A}_{5} \rho+\mathrm{m}_{\mathrm{e}}
\end{aligned}
$$

Nas expressões anteriores $m_{e}$ representa a massa por unidade de comprimento proveniente da escada, cabos e esteiramento, sendo $\mathrm{m}_{\mathrm{e}}=40 \mathrm{~kg} / \mathrm{m}$. Designando a massa distribuída generalizada por $m_{R}$, tem-se

$$
\mathrm{m}_{\mathrm{R}}=\sum_{\mathrm{i}=1}^{5} \mathrm{~m}_{\mathrm{i}}
$$

Com $\mathrm{m}_{0}$ igual a $1097,76 \mathrm{~kg}$, representando a massa oriunda das antenas e demais corpos fixados ao topo da estrutura. A massa generalizada total fica, então:

$$
\mathrm{M}=\mathrm{m}_{0}+\mathrm{m}_{\mathrm{R}}
$$




\subsubsection{Cálculo da rigidez generalizada}

\subsubsection{Rigidez geométrica generalizada}

Para calcular a rigidez geométrica generalizada foi preciso determinar os esforços normais relativos aos trechos definidos na geometria. Do topo para a base da estrutura têm-se:

$$
\begin{aligned}
& \mathrm{F}_{0}=\mathrm{m}_{0} g \\
& \mathrm{~F}_{5}=\int_{\mathrm{L}_{4}}^{\mathrm{L}} \mathrm{m}_{\mathrm{V}} \mathrm{gdx}, \\
& \mathrm{F}_{4}=\int_{\mathrm{L}_{3}}^{\mathrm{L}_{4}} \mathrm{~m}_{\mathrm{IV}}(\mathrm{x}) \mathrm{gdx}, \\
& \mathrm{F}_{3}=\int_{\mathrm{L}_{2}}^{\mathrm{L}_{3}} \mathrm{~m}_{\mathrm{III}} g \mathrm{dx}, \\
& \mathrm{F}_{2}=\int_{\mathrm{L} 1}^{\mathrm{L}_{2}} \mathrm{~m}_{\mathrm{II}} g \mathrm{dx} \mathrm{e} \\
& \mathrm{F}_{1}=\int_{0}^{\mathrm{L}_{1}} \mathrm{~m}_{\mathrm{I}}(\mathrm{x}) \mathrm{gdx} .
\end{aligned}
$$

A força normal generalizada $\mathrm{F}$ será obtida pela soma de suas parcelas,

$$
\mathrm{F}=\sum_{\mathrm{i}=1}^{5} \mathrm{~F}_{\mathrm{i}}
$$

As rigidezes geométricas podem, por fim, serem calculadas pelas seguintes integrais:

$$
\mathrm{K}_{\mathrm{g} 5}=\int_{\mathrm{L}_{4}}^{\mathrm{L}}\left[\mathrm{F}_{0}+\mathrm{m}_{\mathrm{V}}(\mathrm{L}-\mathrm{x}) \mathrm{g}\left(\frac{\mathrm{d}}{\mathrm{dx}} \phi(\mathrm{x})\right)^{2}\right]
$$




$$
\begin{aligned}
& \mathrm{K}_{\mathrm{g} 4}=\int_{\mathrm{L}_{3}}^{\mathrm{L}_{4}}\left[\mathrm{~F}_{0}+\mathrm{F}_{5}+\mathrm{m}_{\mathrm{IV}}(\mathrm{x})\left(\mathrm{L}_{4}-\mathrm{x}\right) \mathrm{g}\left(\frac{\mathrm{d}}{\mathrm{dx}} \phi(\mathrm{x})\right)^{2}\right] \\
& \mathrm{K}_{\mathrm{g} 3}=\int_{\mathrm{L}_{2}}^{\mathrm{L}_{3}}\left[\mathrm{~F}_{0}+\mathrm{F}_{5}+\mathrm{F}_{4}+\mathrm{m}_{\mathrm{III}}\left(\mathrm{L}_{3}-\mathrm{x}\right) \mathrm{g}\left(\frac{\mathrm{d}}{\mathrm{dx}} \phi(\mathrm{x})\right)^{2}\right] \\
& \mathrm{K}_{\mathrm{g} 2}=\int_{\mathrm{L}_{2}}^{\mathrm{L}_{3}}\left[\mathrm{~F}_{0}+\mathrm{F}_{5}+\mathrm{F}_{4}+\mathrm{F}_{3}+\mathrm{m}_{\mathrm{II}}\left(\mathrm{L}_{2}-\mathrm{x}\right) \mathrm{g}\left(\frac{\mathrm{d}}{\mathrm{dx}} \phi(\mathrm{x})\right)^{2}\right] \\
& \mathrm{K}_{\mathrm{g} 2}=\int_{\mathrm{L}_{2}}^{\mathrm{L}_{3}}\left[\mathrm{~F}_{0}+\mathrm{F}_{5}+\mathrm{F}_{4}+\mathrm{F}_{3}+\mathrm{F}_{3}+\mathrm{m}_{\mathrm{I}}(\mathrm{x})\left(\mathrm{L}_{1}-\mathrm{x}\right) \mathrm{g}\left(\frac{\mathrm{d}}{\mathrm{dx}} \phi(\mathrm{x})\right)^{2}\right]
\end{aligned}
$$

A rigidez geométrica generalizada final $\mathrm{K}_{\mathrm{g}}$ é

$$
\mathrm{K}_{\mathrm{g}}=\sum_{\mathrm{i}=1}^{5} \mathrm{~K}_{\mathrm{gi}}
$$

\subsubsection{Rigidez elástica generalizada}

As parcelas da rigidez elástica para os intervalos definidos na geometria são:

$$
\begin{aligned}
& \mathrm{K}_{01}=\int_{0}^{\mathrm{L}_{1}} \mathrm{E}_{1} \mathrm{I}_{1}(\mathrm{x})\left(\frac{\mathrm{d}^{2}}{\mathrm{dx}^{2}} \phi(\mathrm{x})\right)^{2} \mathrm{dx} \\
& \mathrm{K}_{02}=\int_{\mathrm{L}_{1}}^{\mathrm{L}_{2}} \mathrm{E}_{1} \mathrm{I}_{2}\left(\frac{\mathrm{d}^{2}}{\mathrm{dx}^{2}} \phi(\mathrm{x})\right)^{2} \mathrm{dx} \\
& \mathrm{K}_{03}=\int_{\mathrm{L}_{2}}^{\mathrm{L}_{3}} \mathrm{EI}_{3}\left(\frac{\mathrm{d}^{2}}{\mathrm{dx}^{2}} \phi(\mathrm{x})\right)^{2} \mathrm{dx}
\end{aligned}
$$




$$
\begin{aligned}
& \mathrm{K}_{04}=\int_{\mathrm{L}_{3}}^{\mathrm{L}_{4}} \mathrm{EI}_{4}(\mathrm{x})\left(\frac{\mathrm{d}^{2}}{\mathrm{dx}^{2}} \phi(\mathrm{x})\right)^{2} \mathrm{dx} \\
& \mathrm{K}_{05}=\int_{\mathrm{L}_{4}}^{\mathrm{L}} \mathrm{EI}_{5}\left(\frac{\mathrm{d}^{2}}{\mathrm{dx}^{2}} \phi(\mathrm{x})\right)^{2} \mathrm{dx}
\end{aligned}
$$

$\mathrm{E}$ a rigidez elástica generalizada $\mathrm{K}_{0}$ é, portanto:

$$
\mathrm{K}_{0}=\sum_{\mathrm{i}=1}^{5} \mathrm{~K}_{0 \mathrm{i}}
$$

\subsubsection{Rigidez das molas generalizada}

Para explorar todos os recursos e avaliar a potencialidade do método proposto neste trabalho de pesquisa fez-se a introdução de molas distribuídas para representar o solo lateral.

Chamando de $\mathrm{k}_{\mathrm{s}}$ o fator de mola, cujo valor, para o caso específico, é $\mathrm{k}_{\mathrm{s}}=2669 \frac{\mathrm{kN}}{\mathrm{m}^{3}}$, as molas distribuídas, no primeiro e no segundo trecho do fuste, são dadas por $\mathrm{k}_{1}(\mathrm{x})=\mathrm{k}_{\mathrm{s}} \mathrm{D}_{1}(\mathrm{x})$ e $\mathrm{k}_{2}(\mathrm{x})=\mathrm{k}_{\mathrm{s}} \mathrm{D}_{2}$. A rigidez generalizada das molas, designada por $\mathrm{K}_{\mathrm{m}}$, foi calculada por meio da seguinte expressão:

$$
\mathrm{K}_{\mathrm{m}}=\int_{0}^{\mathrm{L}_{1}} \mathrm{k}_{1}(\mathrm{x}) \phi(\mathrm{x})^{2} \mathrm{dx}+\int_{\mathrm{L}_{1}}^{\mathrm{L}_{2}} \mathrm{k}_{2}(\mathrm{x}) \phi(\mathrm{x})^{2} \mathrm{dx}
$$

A rigidez generalizada $\mathrm{K}$ da estrutura pode então ser determinada pela soma algébrica das parcelas calculadas nos subitens precedentes, de forma que se obtém:

$$
\mathrm{K}=\mathrm{K}_{0}-\mathrm{K}_{\mathrm{g}}+\mathrm{K}_{\mathrm{m}}
$$




\subsubsection{Cálculo da frequência}

Para o modelo de $46 \mathrm{~m}$, o valor da frequência fundamental com a não-linearidade geométrica e material é de 0,145517 Hz. Uma análise exclusivamente linear pelo método proposto fornece uma frequência para o primeiro modo de $0,218848 \mathrm{~Hz}$.

Já para o modelo de $40 \mathrm{~m}$, tem-se, com ambas as não-linearidades, a frequência de $0,195532 \mathrm{~Hz}$, e para o modelo exclusivamente linear, a frequência de 0,285602 Hz.

\subsubsection{Ação do vento}

\subsubsection{Forças estáticas devidas ao vento}

As forças estáticas devidas à ação do vento foram calculadas como descrito no item 6.1. Os parâmetros empregados na análise foram: fator topográfico $S_{1}=1,1$; fator de rugosidade do terreno $S_{2}$ correspondente à categoria IV, classe $\mathrm{B}$, calculado conforme a expressão (6.2) com os parâmetros $\mathrm{p}=0,125, \mathrm{~b}=0,85$ e $\mathrm{F}_{\mathrm{r}}=0,98$, presentes na Tabela 6.1; fator estatístico $\mathrm{S}_{3}=1,1$; velocidade básica do vento $\mathrm{V}_{0}=$ $35 \mathrm{~m} / \mathrm{s}$ e altura sobre o solo de $40 \mathrm{~m}$.

\subsubsection{Resposta dinâmica pelo modelo simplificado da NBR 6123/88}

Na determinação da resposta dinâmica pelo modelo contínuo simplificado foram empregados os seguintes parâmetros: largura da edificação $0,723 \mathrm{~m}$; altura da edificação de $46 \mathrm{~m}$ para o cálculo da frequência; categoria do terreno IV; velocidade básica $35 \mathrm{~m} / \mathrm{s}$; fatores estatísticos $\mathrm{S}_{1}$ e $\mathrm{S}_{3}$ iguais a 1,1 .

A frequência fundamental foi obtida fazendo $\mathrm{T}_{1}=0,02 \mathrm{~h}$ (Tabela 6.4), $\operatorname{logo} \mathrm{f}_{1}=1,086957 \mathrm{~Hz}$. É interessante ressaltar que a frequência fundamental da estrutura, como calculada, ficou acima de $1 \mathrm{~Hz}$, o que poderia induzir ao engenheiro a eximir-se do cálculo da resposta dinâmica da estrutura, conforme previsto no capítulo 9 da NBR 6123/88. 
A forma modal obedeceu à expressão (6.6) com g igual a 2,7. Conhecida a velocidade de projeto e a frequência natural da estrutura, obteve-se a relação adimensional Vp/( $\left.f_{1} L\right)$ de 0,013 , o que leva, com uma taxa crítica de amortecimento $\zeta$ igual a 0,015 , a um coeficiente de amplificação dinâmica $\xi$ de 1,131.

No cálculo da resposta dinâmica com base no modelo contínuo simplificado da NBR 6123/88, com a inclusão da não-linearidade geométrica e material, a relação adimensional $\mathrm{Vp} /\left(\mathrm{f}_{1} \mathrm{~L}\right)$ é de 0,115 . Com os ábacos da NBR 6123/88, e para o mesmo amortecimento crítico, obtém-se $\xi=2,758$.

\subsubsection{Resposta dinâmica pelo modelo discreto da NBR $6123 / 88$}

Calculando a frequência pelo Método dos Elementos Finitos encontra-se uma frequência para o modo fundamental de 0,216915 Hz. A relação adimensional $\mathrm{Vp} /\left(\mathrm{f}_{1} \mathrm{~L}\right)$ passa a ser 0,075 , o que conduz a um fator de amplificação dinâmica $\xi$ de 1,702, considerando o mesmo amortecimento crítico.

Os parâmetros usados no cálculo da resposta dinâmica discreta, com a inclusão da não-linearidade geométrica e material, são descrito na análise simplificada não-linear, apresentados no item anterior. Para os modos de 2 a 5 os fatores de amplificação dinâmica, dadas as frequências apresentadas na Figura 7.22, são, respectivamente: 1,$796 ; 1,492 ; 1,321 ; 1,321$.

\subsubsection{Análise dos resultados}

As diferenças no valor do esforço normal entre o método proposto e o Método dos Elementos Finitos, nas posições de interesse definidas na geometria da estrutura, podem ser encontrada na Tabela 7.18. 
Tabela 7.18 - Estrutura 4: esforço normal.

\begin{tabular}{|c|c|c|c|c|}
\hline $\mathbf{L}$ & Proposto & MEF & \multicolumn{2}{|c|}{ Diferença } \\
\hline $\mathbf{( m )}$ & $\mathbf{( k N )}$ & $\mathbf{( k N )}$ & Absoluta & $\mathbf{( \% )}$ \\
\hline 46,00 & 10,758048 & 10,758048 & 0,0000 & 0,0000000 \\
\hline 19,00 & 181,493643 & 181,493637 & 0,0000 & 0,0000034 \\
\hline 12,00 & 234,667745 & 234,667737 & 0,0000 & 0,0000035 \\
\hline 6,00 & 283,847697 & 283,847687 & 0,0000 & 0,0000035 \\
\hline 0,20 & 355,274947 & 355,274935 & 0,0000 & 0,0000036 \\
\hline 0,00 & 360,277934 & 360,277921 & 0,0000 & 0,0000036 \\
\hline
\end{tabular}

A frequência do primeiro modo, obtida pelo método proposto, como descrito no item 7.4.3, foi de 0,145517 Hz. Quando calculada pelo Método dos Elementos Finitos essa mesma frequência é de 0,141285 Hz, o que corresponde a uma diferença de apenas 2,99\%. Um cálculo simplificado pelo método proposto pôde ser feito utilizando-se as propriedades geométricas e materiais da estrutura de forma ponderada. Assim, mantendo-se a rigidez elástica das molas na formulação, encontra-se um valor de $0,115210 \mathrm{~Hz}$, o que representa uma diferença de 20,83\% em relação ao cálculo exato; de 18,46\% em relação ao mesmo modelo pelo MEF e de 89,40\% em relação à prescrição da NBR 6123/88.

No entanto, para usar diretamente a expressão (4.19) é preciso considerar a estrutura simplesmente engastada na superfície do terreno. Com essa hipótese, o cálculo simplificado pelo método proposto, incluindo a não-linearidade do concreto, conduz à frequência de $0,195532 \mathrm{~Hz}$, distante $34,37 \%$ do valor anterior. Pelo MEF, com o modelo correspondente, obtém-se 0,205239 Hz, o que significa uma diferença de 4,73\%, e pela NBR 6123/88 encontra-se 1,25 Hz, representando uma diferença de $84,36 \%$. 
Acompanhando os resultados já encontrados para as estruturas anteriores, os valores críticos para resposta dinâmica da estrutura sob ação do vento encontram-se na comparação entre o modelo estático e o modelo dinâmico discreto simplificado não-linear. A diferença do momento fletor na estrutura entre essas duas análises atinge o valor máximo de 53\%.

Já a resposta dinâmica obtida com o modelo simplificado conduz ao momento máximo 1,18 vezes maior do que o encontrado nos cálculos com o modelo de forças estáticas.

Nas análises discretas não-lineares segue-se com uma pequena influência da contribuição dos modos de vibração acima do fundamental. Quando computadas as contribuições do vento médio e as flutuações do $1^{\circ}$ ao $5^{\circ}$ modos surge uma diferença de 0,01\%, portanto desprezível, em relação à combinação que leva em conta apenas a ação do vento médio com a contribuição do primeiro modo de vibração.

As flutuações resultantes do primeiro modo de vibração são as que aportam maior contribuição na resposta da estrutura, representando $60 \%$ do valor total. Analisando os resultados obtidos na resposta dinâmica dos modelos discretos, linear e não-linear, observa-se uma diferença na frequência do primeiro modo de 35 \% e no fator de amplificação dinâmica de 8,06\%. Com isso, o momento fletor máximo na estrutura ficou acrescido de $38,99 \mathrm{kNm}$, o que equivale a uma força de $1 \mathrm{kN}$ aplicada no topo. Na Tabela 7.19 constam os resultados da análise dinâmica discreta não-linear.

A diferença entre a análise discreta não-linear e análise das forças estáticas, ou modelo estático, é de 8,33\%, o que reduz a capacidade da estrutura em possuir área de exposição ao vento, uma vez que seu projeto foi realizado com os esforços do vento sendo computados pelo último processo.

A Tabela 7.20 resume os valores dos momentos na estrutura devidos à ação do vento das análises descritas anteriormente, comparando-as ao modelo estático. 
Tabela 7.19 - Estrutura 4: momentos da análise discreta nãolinear $(\zeta=0,015)$.

\begin{tabular}{|c|c|c|c|c|c|c|}
\hline \multirow{2}{*}{ z } & \multirow{2}{*}{$\begin{array}{l}\text { Vento } \\
\text { Médio }\end{array}$} & \multicolumn{5}{|c|}{ Flutuações } \\
\hline & & Modo1 & Modo 2 & Modo 3 & Modo 4 & Modo 5 \\
\hline (m) & (kNm) & (kNm) & (kNm) & (kNm) & (kNm) & (kNm) \\
\hline 40 & 0,00 & 0,00 & 0,00 & 0,00 & 0,00 & 0,00 \\
\hline 39 & 6,23 & 2,76 & 0,32 & 0,54 & 0,30 & 0,28 \\
\hline 38 & 12,78 & 6,72 & 0,76 & 1,28 & 0,69 & 0,62 \\
\hline 37 & 19,65 & 11,86 & 1,31 & 2,15 & 1,13 & 0,97 \\
\hline 36 & 26,84 & 18,12 & 1,95 & 3,11 & 1,56 & 1,28 \\
\hline 35 & 34,35 & 25,47 & 2,66 & 4,10 & 1,96 & 1,51 \\
\hline 34 & 42,16 & 33,86 & 3,43 & 5,07 & 2,28 & 1,62 \\
\hline 33 & 50,28 & 43,25 & 4,23 & 5,97 & 2,51 & 1,60 \\
\hline 32 & 58,71 & 53,61 & 5,06 & 6,77 & 2,61 & 1,44 \\
\hline 31 & 67,43 & 64,88 & 5,89 & 7,44 & 2,58 & 1,16 \\
\hline 30 & 76,44 & 77,04 & 6,70 & 7,93 & 2,42 & 0,78 \\
\hline 29 & 85,74 & 90,03 & 7,49 & 8,23 & 2,12 & 0,33 \\
\hline 28 & 95,33 & 103,82 & 8,24 & 8,32 & 1,72 & 0,15 \\
\hline
\end{tabular}




\begin{tabular}{|c|c|c|c|c|c|c|}
\hline \multirow{2}{*}{ z } & \multirow{2}{*}{$\begin{array}{l}\text { Vento } \\
\text { Médio }\end{array}$} & \multicolumn{5}{|c|}{ Flutuações } \\
\hline & & Modo1 & Modo 2 & Modo 3 & Modo 4 & Modo 5 \\
\hline (m) & (kNm) & (kNm) & (kNm) & (kNm) & (kNm) & (kNm) \\
\hline 27 & 105,19 & 118,36 & 8,93 & 8,19 & 1,22 & 0,61 \\
\hline 26 & 115,33 & 133,63 & 9,55 & 7,85 & 0,66 & 1,01 \\
\hline 25 & 125,74 & 149,57 & 10,10 & 7,29 & 0,07 & 1,31 \\
\hline 24 & 136,42 & 166,16 & 10,55 & 6,53 & 0,53 & 1,49 \\
\hline 23 & 147,35 & 183,34 & 10,91 & 5,59 & 1,09 & 1,52 \\
\hline 22 & 158,55 & 201,10 & 11,16 & 4,49 & 1,58 & 1,41 \\
\hline 21 & 169,99 & 219,37 & 11,30 & 3,27 & 1,97 & 1,17 \\
\hline 20 & 181,68 & 238,15 & 11,32 & 1,95 & 2,25 & 0,81 \\
\hline 19 & 193,60 & 257,38 & 11,22 & 0,58 & 2,39 & 0,38 \\
\hline 18 & 205,76 & 277,03 & 11,00 & 0,80 & 2,39 & 0,09 \\
\hline 17 & 218,15 & 297,07 & 10,65 & 2,16 & 2,24 & 0,55 \\
\hline 16 & 230,76 & 317,48 & 10,17 & 3,46 & 1,96 & 0,97 \\
\hline 15 & 243,59 & 338,21 & 9,57 & 4,67 & 1,55 & 1,29 \\
\hline 14 & 256,63 & 359,24 & 8,85 & 5,74 & 1,05 & 1,50 \\
\hline
\end{tabular}




\begin{tabular}{|c|c|c|c|c|c|c|}
\hline \multirow{2}{*}{$\mathbf{z}$} & \multirow{2}{*}{$\begin{array}{l}\text { Vento } \\
\text { Médio }\end{array}$} & \multicolumn{5}{|c|}{ Flutuações } \\
\hline & & Modo1 & Modo 2 & Modo 3 & Modo 4 & Modo 5 \\
\hline (m) & (kNm) & (kNm) & (kNm) & (kNm) & (kNm) & (kNm) \\
\hline 13 & 269,87 & 380,54 & 8,02 & 6,65 & 0,47 & 1,57 \\
\hline 12 & 283,31 & 402,09 & 7,06 & 7,36 & 0,15 & 1,49 \\
\hline 11 & 296,94 & 423,90 & 5,99 & 7,85 & 0,78 & 1,26 \\
\hline 10 & 310,75 & 445,93 & 4,80 & 8,09 & 1,39 & 0,91 \\
\hline 9 & 324,75 & 468,17 & 3,50 & 8,07 & 1,93 & 0,45 \\
\hline 8 & 338,92 & 490,59 & 2,09 & 7,77 & 2,38 & 0,06 \\
\hline 7 & 353,25 & 513,17 & 0,60 & 7,21 & 2,70 & 0,58 \\
\hline 6 & 367,75 & 535,90 & 0,99 & 6,38 & 2,87 & 1,06 \\
\hline 5 & 382,39 & 558,75 & 2,65 & 5,30 & 2,88 & 1,45 \\
\hline 4 & 397,18 & 581,71 & 4,38 & 3,99 & 2,72 & 1,72 \\
\hline 3 & 412,09 & 604,75 & 6,16 & 2,46 & 2,39 & 1,83 \\
\hline 2 & 427,07 & 627,86 & 7,99 & 0,76 & 1,90 & 1,78 \\
\hline 1 & 442,11 & 651,02 & 9,86 & 1,09 & 1,27 & 1,55 \\
\hline 0 & 457,20 & 674,22 & 11,76 & 3,06 & 0,51 & 1,15 \\
\hline
\end{tabular}




\begin{tabular}{|c|c|c|c|c|c|c|c|c|c|c|}
\hline \multicolumn{2}{|c|}{$\begin{array}{l}\text { 岕 } \\
\text { 呈 }\end{array}$} & & $\sum_{\underline{\underline{E}}}^{\overline{\underline{\varepsilon}}}$ & $\begin{array}{l}\circ \\
\text { ○ } \\
\end{array}$ & $\stackrel{\text { న }}{\stackrel{\text { D }}{N}}$ & $\begin{array}{l}\hat{0} \\
\text { No }\end{array}$ & $\begin{array}{l}\infty \\
\text { D } \\
\text { D" }\end{array}$ & $\begin{array}{l}\infty \\
\circ \\
\text { 。' } \\
\text { ○ }\end{array}$ & $\begin{array}{l}\text { ా } \\
\text { गे } \\
\text { - }\end{array}$ & 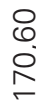 \\
\hline \multicolumn{2}{|c|}{ 㟔 } & & ${ }_{\underline{\underline{E}}}^{\overline{\underline{E}}}$ & $\begin{array}{l}8 \\
\text { ○ }\end{array}$ & 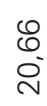 & $\begin{array}{l}\stackrel{\mathrm{L}}{\stackrel{2}{\sim}} \\
\stackrel{\sim}{*}\end{array}$ & $\begin{array}{l}5 \\
0 \\
0 \\
0\end{array}$ & $\begin{array}{l}\text { శ } \\
\infty \\
\infty \\
\infty\end{array}$ & $\begin{array}{l}m \\
\text { m} \\
\stackrel{5}{\sigma}\end{array}$ & $\begin{array}{l}\text { N } \\
\infty \\
\stackrel{\sim}{\sim}\end{array}$ \\
\hline \multirow{5}{*}{ 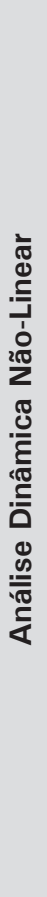 } & \multirow{5}{*}{ 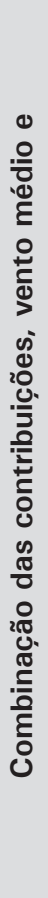 } & $\begin{array}{l}\text { م } \\
\text { ס } \\
\end{array}$ & $\bar{\sum}_{\underline{\underline{E}}}$ & $\begin{array}{l}8 \\
0 \\
0\end{array}$ & $\begin{array}{l}\infty \\
\infty \\
\infty \\
\infty\end{array}$ & $\begin{array}{l}\text { ণ } \\
\text { の }\end{array}$ & $\frac{\infty}{+}$ & $\begin{array}{l}\infty \\
\dot{J}\end{array}$ & 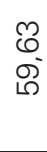 & $\frac{0}{\circ}$ \\
\hline & & $\begin{array}{l}\dot{\sigma} \\
\sigma \\
\sigma\end{array}$ & $\sum_{\underline{\underline{E}}}^{\bar{\xi}}$ & $\begin{array}{l}\text { ০ } \\
\text { ' }\end{array}$ & $\begin{array}{l}\hat{\sigma} \\
\infty\end{array}$ & $\begin{array}{l}\text { f } \\
\text { ळ் }\end{array}$ & $\frac{\text { f }}{\dot{m}}$ & $\begin{array}{l}\text { O } \\
\text { J }\end{array}$ & 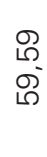 & $\begin{array}{l}\hat{\theta} \\
\text { L }\end{array}$ \\
\hline & & $\begin{array}{l}m \\
\pi \\
\sigma\end{array}$ & $\sum_{\underline{\underline{E}}}^{\bar{\xi}}$ & $\begin{array}{l}8 \\
0 \\
0\end{array}$ & $\begin{array}{l}\stackrel{\llcorner}{\Omega} \\
\infty \\
\infty\end{array}$ & $\begin{array}{l}\stackrel{m}{+} \\
\stackrel{-}{\sigma}\end{array}$ & $\begin{array}{l}\text { m } \\
\text { m }\end{array}$ & $\begin{array}{r}\text { 尺 } \\
\text { J }\end{array}$ & $\begin{array}{l}\text { గ్ } \\
\text { م' }\end{array}$ & 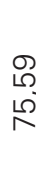 \\
\hline & & $\begin{array}{l}N \\
0 \\
\sigma\end{array}$ & $\underline{\underline{\underline{\xi}}}$ & $\begin{array}{l}\text { O } \\
\text { ' }\end{array}$ & $\begin{array}{l}\text { ᄋి } \\
\infty\end{array}$ & $\begin{array}{l}\bar{m} \\
\bar{\sigma}\end{array}$ & $\frac{\stackrel{丶}{N}}{\stackrel{m}{m}}$ & \begin{tabular}{l}
$\bar{\Sigma}$ \\
\multirow{8}{*}{}
\end{tabular} & $\begin{array}{l}\stackrel{\curvearrowright}{N} \\
\text { D' }\end{array}$ & \begin{tabular}{l} 
N \\
\multirow{N}{N}{}
\end{tabular} \\
\hline & & $\begin{array}{l}\overline{0} \\
\text { 음 } \\
\text { L }\end{array}$ & $\underset{\underline{\underline{\xi}}}{\sum_{\underline{\underline{z}}}}$ & $\begin{array}{l}\text { ○ } \\
\text { - }\end{array}$ & $\begin{array}{l}\infty \\
\infty \\
\infty\end{array}$ & $\begin{array}{l}\hat{N} \\
\stackrel{\sigma}{\sigma}\end{array}$ & $\frac{m}{\bar{m}}$ & 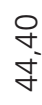 & $\begin{array}{l}\text { ○ } \\
\text { م' }\end{array}$ & $\begin{array}{l}\stackrel{\llcorner}{\circ} \\
\stackrel{\circ}{N}\end{array}$ \\
\hline \multicolumn{2}{|c|}{ 号 } & & $\underset{\underline{\underline{E}}}{\overline{\underline{E}}}$ & ০০ & $\underset{\infty}{-}$ & $\begin{array}{l}\stackrel{\llcorner}{\infty} \\
\infty \\
\infty\end{array}$ & $\begin{array}{l}\hat{m} \\
\hat{0}\end{array}$ & 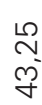 & 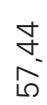 & $\begin{array}{l}\text { ᄋ } \\
\text { N }\end{array}$ \\
\hline 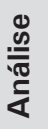 & 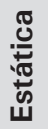 & & $\begin{array}{l}\bar{\varepsilon} \\
\underline{\underline{E}}\end{array}$ & $\begin{array}{l}8 \\
0 \\
\end{array}$ & $\begin{array}{l}\text { Q } \\
\text { ஸ் }\end{array}$ & $\stackrel{\bar{v}}{\stackrel{N}{N}}$ & $\begin{array}{l}\infty \\
\stackrel{+}{\sim} \\
\stackrel{+}{*}\end{array}$ & $\begin{array}{l}\text { ठ } \\
00 \\
0 \\
10\end{array}$ & \begin{tabular}{l} 
m \\
\multirow{N}{*}{}
\end{tabular} & $\frac{\grave{N}}{\sigma}$ \\
\hline & $\mathbf{N}$ & & $\underline{\xi}$ & 우 & ল & $\stackrel{\infty}{m}$ & $\hat{m}$ & ले & $\stackrel{\stackrel{m}{m}}{ }$ & ল \\
\hline
\end{tabular}




\begin{tabular}{|c|c|c|c|c|c|c|c|c|c|c|c|}
\hline \multicolumn{2}{|c|}{$\begin{array}{l}\text { Z } \\
\text { 足 } \\
\text { 是 }\end{array}$} & & $\begin{array}{l}\overline{\underline{\xi}} \\
\underline{\underline{z}}\end{array}$ & $\begin{array}{l}\text { D } \\
\text { N } \\
\text { D }\end{array}$ & $\begin{array}{l}\stackrel{N}{N} \\
0 \\
\stackrel{N}{N}\end{array}$ & \begin{tabular}{l}
0 \\
\multirow{2}{*}{} \\
$\stackrel{0}{N}$
\end{tabular} & 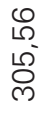 & $\frac{\text { 号 }}{\text { 㐫 }}$ & $\begin{array}{l}\underset{N}{N} \\
\infty \\
\stackrel{0}{\infty}\end{array}$ & $\begin{array}{l}00 \\
@ \\
\omega^{\circ} \\
\bar{\forall}\end{array}$ & 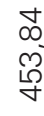 \\
\hline \multicolumn{2}{|c|}{ 号 } & & $\begin{array}{l}\overline{\underline{\underline{\varepsilon}}} \\
\underline{\underline{\underline{z}}}\end{array}$ & $\begin{array}{l}\hat{N} \\
\tilde{+} \\
\stackrel{0}{-}\end{array}$ & $\begin{array}{l}\infty \\
\stackrel{2}{ } \\
\stackrel{-}{\circ} \\
\stackrel{-}{\circ}\end{array}$ & $\begin{array}{l}\text { m } \\
\infty 0^{-} \\
\stackrel{\sim}{N}\end{array}$ & 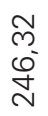 & 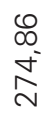 & $\begin{array}{l}\text { m. } \\
\text { ले } \\
\text { ले }\end{array}$ & $\begin{array}{l}\text { P } \\
\text { ले } \\
\text { ले }\end{array}$ & 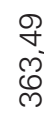 \\
\hline \multirow{5}{*}{ 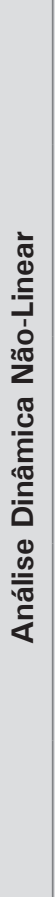 } & \multirow{5}{*}{ 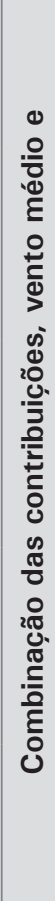 } & $\begin{array}{l}\text { م } \\
\text { ๘ } \\
-\end{array}$ & 忘 & $\begin{array}{l}\text { D } \\
\text { ल }\end{array}$ & $\begin{array}{l}8 \\
\check{E} \\
\mp\end{array}$ & $\begin{array}{l}\dot{m} \\
\dot{m} \\
\stackrel{m}{-}\end{array}$ & $\begin{array}{l}\underset{N}{N} \\
\underset{N}{6}\end{array}$ & $\begin{array}{l}\stackrel{ }{N} \\
\stackrel{\mathbb{N}}{上}\end{array}$ & $\begin{array}{l}\stackrel{+}{\sim} \\
\stackrel{\text { Oे }}{\sim}\end{array}$ & 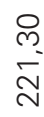 & $\begin{array}{l}M \\
m \\
0 \\
\stackrel{0}{\sim}\end{array}$ \\
\hline & & $\begin{array}{l}\dot{\sigma} \\
\sigma \\
-\end{array}$ & $\underset{\underline{\underline{\underline{E}}}}{\overline{\underline{E}}}$ & $\begin{array}{l}\bar{\sigma} \\
\text { ळ్ }\end{array}$ & $\begin{array}{l}\stackrel{\infty}{\llcorner} \\
\stackrel{\Sigma}{\mp}\end{array}$ & 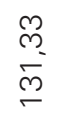 & \begin{tabular}{l}
$\underset{N}{N}$ \\
\multirow{N}{\sim}{} \\
$\stackrel{n}{\leftarrow}$
\end{tabular} & 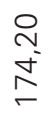 & 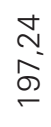 & 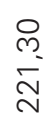 & $\begin{array}{l}\stackrel{m}{m} \\
0 \\
\stackrel{0}{+} \\
\sim\end{array}$ \\
\hline & & $\begin{array}{l}m \\
\text { o } \\
-\end{array}$ & $\begin{array}{l}\overline{\underline{\varepsilon}} \\
\text { 兰 }\end{array}$ & $\begin{array}{l}\text { Dे } \\
\text { N }\end{array}$ & 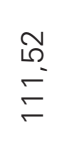 & $\begin{array}{l}\stackrel{\infty}{v} \\
\stackrel{m}{m}\end{array}$ & 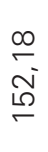 & $\begin{array}{l}\stackrel{\infty}{\nwarrow} \\
\stackrel{+}{\approx}\end{array}$ & $\begin{array}{l}\stackrel{m}{N} \\
\stackrel{\text { On}}{\sigma}\end{array}$ & $\begin{array}{l}\stackrel{D}{N} \\
\underset{N}{N}\end{array}$ & $\begin{array}{l}\stackrel{m}{m} \\
\stackrel{0}{0} \\
\stackrel{\sim}{\sim}\end{array}$ \\
\hline & & $\begin{array}{l}N \\
0 \\
-\end{array}$ & $\begin{array}{l}\overline{\underline{\varepsilon}} \\
\underline{\underline{\Sigma}}\end{array}$ & $\begin{array}{l}\text { Lิ } \\
\text { న }\end{array}$ & $\begin{array}{l}\stackrel{ }{\mp} \\
\mp\end{array}$ & $\begin{array}{l}0 \\
\infty \\
0 \\
0 \\
\end{array}$ & $\frac{\infty}{\stackrel{\infty}{\Sigma}}$ & $\begin{array}{l}\bar{\infty} \\
\stackrel{\infty}{\stackrel{1}{\alpha}}\end{array}$ & 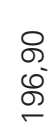 & $\frac{\overline{-}}{\grave{N}}$ & \begin{tabular}{l}
$\stackrel{\circ}{0}$ \\
$\stackrel{0}{+}$ \\
\multirow{2}{*}{}
\end{tabular} \\
\hline & & $\begin{array}{l}\overline{0} \\
\stackrel{0}{\circ} \\
\Sigma\end{array}$ & $\begin{array}{l}\bar{\xi} \\
\sum_{\underline{\underline{E}}}\end{array}$ & $\begin{array}{l}\text { W } \\
\text { N } \\
\text {. }\end{array}$ & $\begin{array}{l}\infty \\
\infty \\
0 \\
\varnothing \\
\varnothing\end{array}$ & $\begin{array}{l}8 \\
\stackrel{8}{0} \\
\text { m. }\end{array}$ & $\begin{array}{l}\text { g } \\
\frac{5}{5}\end{array}$ & 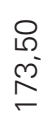 & $\begin{array}{l}\infty \\
\stackrel{\circ}{\Omega} \\
\stackrel{\circ}{\circ} \\
\stackrel{\mathscr{D}}{\sigma}\end{array}$ & 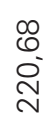 & $\begin{array}{l}\stackrel{0}{\sim} \\
\stackrel{5}{\sim} \\
\sim\end{array}$ \\
\hline \multicolumn{2}{|c|}{$\overrightarrow{\mathrm{Q}}$} & & $\begin{array}{l}\overline{\underline{\varepsilon}} \\
\underline{\underline{\underline{E}}}\end{array}$ & $\begin{array}{l}\text { 乃 } \\
\stackrel{0}{\circ} \\
\infty\end{array}$ & $\begin{array}{l}\text { f } \\
\text { 음 }\end{array}$ & $\begin{array}{l}\stackrel{\circ}{\llcorner} \\
0 \\
\stackrel{0}{\sim}\end{array}$ & 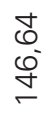 & 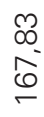 & $\begin{array}{l}8 \\
\stackrel{0}{\circ} \\
\stackrel{8}{\circ}\end{array}$ & $\begin{array}{l}\stackrel{0}{N} \\
\stackrel{m}{\sim} \\
\stackrel{\sim}{N}\end{array}$ & $\begin{array}{l}\overline{+} \\
\hat{\sim} \\
\stackrel{N}{N}\end{array}$ \\
\hline 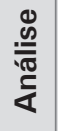 & 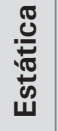 & & $\begin{array}{l}\overline{\underline{\xi}} \\
\text { 兰 }\end{array}$ & $\begin{array}{l}\text { o. } \\
0^{\circ} \\
\stackrel{0}{0}\end{array}$ & $\stackrel{\infty}{\stackrel{\infty}{\approx}}$ & $\begin{array}{l}0 \\
\stackrel{0}{0} \\
\stackrel{\sigma}{\sigma}\end{array}$ & 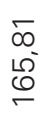 & $\begin{array}{l}\stackrel{1}{2} \\
\stackrel{0}{0} \\
\varnothing\end{array}$ & 定 & $\begin{array}{l}\bar{\lambda} \\
\infty \\
\stackrel{N}{N}\end{array}$ & $\begin{array}{l}\hat{\sigma} \\
\circ \\
\stackrel{\circ}{\circ}\end{array}$ \\
\hline & $N$ & & $\bar{\xi}$ & $m$ & $\widetilde{ల}$ & $\bar{m}$ & ले & $\stackrel{\sim}{N}$ & $\stackrel{\infty}{\sim}$ & 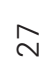 & $\stackrel{i}{\sim}$ \\
\hline
\end{tabular}




\begin{tabular}{|c|c|c|c|c|c|c|c|c|c|c|c|}
\hline \multicolumn{2}{|c|}{$\begin{array}{l}\sum^{3} \\
\text { 足 }\end{array}$} & & ${ }_{\underline{\underline{E}}}^{\overline{\underline{E}}}$ & $\begin{array}{l}\text { ๑ } \\
\text { N } \\
\text { જ }\end{array}$ & 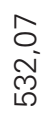 & $\begin{array}{l}0 \\
0 \\
N \\
\text { No }\end{array}$ & 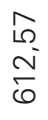 & 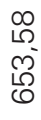 & 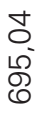 & $\begin{array}{l}\text { నे } \\
\text { లే } \\
\text { న }\end{array}$ & $\frac{\infty}{\infty}$ \\
\hline \multicolumn{2}{|c|}{ 虫 } & & $\begin{array}{l}\overline{\underline{E}} \\
\underline{\underline{\Sigma}}\end{array}$ & $\begin{array}{l}\bar{\sigma} \\
\text { ळ' } \\
\text { ल) }\end{array}$ & 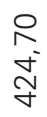 & 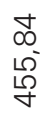 & 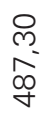 & 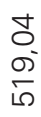 & $\begin{array}{l}m \\
0 \\
5 \\
0 \\
0\end{array}$ & $\begin{array}{l}\stackrel{\omega}{N} \\
\widetilde{N} \\
\infty \\
\infty\end{array}$ & $\begin{array}{l}\infty \\
0 \\
\sigma \\
\sigma\end{array}$ \\
\hline \multirow{5}{*}{ 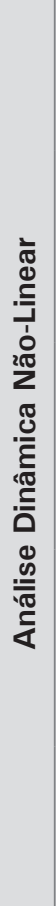 } & \multirow{5}{*}{ 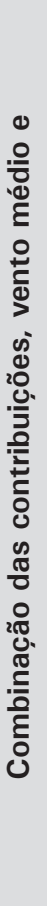 } & $\begin{array}{l}\text { م) } \\
\sigma \\
\sigma\end{array}$ & $\begin{array}{l}\overline{\underline{\xi}} \\
\underline{\underline{z}}\end{array}$ & 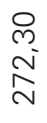 & $\begin{array}{l}\text { ন } \\
\text { ন }\end{array}$ & 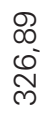 & 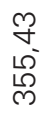 & $\begin{array}{l}\stackrel{+}{N} \\
\text { D } \\
\text { m }\end{array}$ & $\begin{array}{l}\stackrel{\Omega}{\curvearrowright} \\
\frac{\sigma}{\sigma}\end{array}$ & 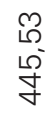 & 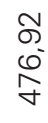 \\
\hline & & $\begin{array}{l}\nabla \\
\sigma \\
\sigma\end{array}$ & $\begin{array}{l}\overline{\underline{\xi}} \\
\underline{\underline{z}}\end{array}$ & 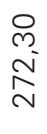 & $\begin{array}{l}0 \\
\stackrel{D}{\sigma} \\
\stackrel{2}{N}\end{array}$ & $\begin{array}{l}\infty \\
\infty \\
0 \\
0 \\
\text { లn }\end{array}$ & 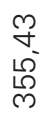 & 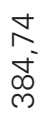 & 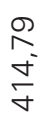 & 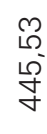 & 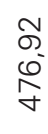 \\
\hline & & $\begin{array}{l}m \\
\sigma \\
\sigma\end{array}$ & ${ }_{\underline{\underline{E}}}^{\overline{\underline{E}}}$ & 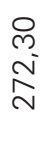 & $\begin{array}{l}0 \\
\text { ब) } \\
\stackrel{N}{2}\end{array}$ & 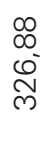 & 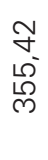 & $\begin{array}{l}\stackrel{m}{N} \\
\stackrel{j}{\infty} \\
\stackrel{m}{m}\end{array}$ & 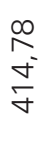 & 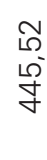 & \begin{tabular}{l}
$\bar{\sigma}$ \\
0 \\
\multirow{\gamma}{\sigma}{}
\end{tabular} \\
\hline & & $\begin{array}{l}N \\
0 \\
-\end{array}$ & $\begin{array}{l}\bar{\xi} \\
\text { 立 }\end{array}$ & $\frac{\sim}{\stackrel{N}{N}}$ & $\begin{array}{l}\text { 寸 } \\
\text { D } \\
\stackrel{N}{N}\end{array}$ & $\begin{array}{l}8 \\
\infty \\
0 \\
0 \\
\text { లె }\end{array}$ & $\begin{array}{l}\hat{m} \\
\text { L } \\
\stackrel{\rho}{m}\end{array}$ & $\begin{array}{l}\bar{\gamma} \\
\dot{j} \\
\infty \\
\infty\end{array}$ & $\begin{array}{l}\text { 尺े } \\
\frac{8}{+}\end{array}$ & 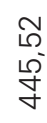 & $\begin{array}{l}\bar{\sigma} \\
0^{\circ} \\
\gamma\end{array}$ \\
\hline & & $\begin{array}{l}- \\
\frac{0}{0} \\
\Sigma \\
\Sigma\end{array}$ & $\begin{array}{l}\bar{\xi} \\
\underline{\underline{E}}\end{array}$ & $\frac{\text { D }}{\stackrel{N}{N}}$ & $\begin{array}{l}\bar{N} \\
\infty \\
\stackrel{\sim}{N}\end{array}$ & \begin{tabular}{l}
$\infty$ \\
$\stackrel{\infty}{+}$ \\
\multirow{2}{*}{} \\
\end{tabular} & $\begin{array}{l}0 \\
0 \\
10 \\
\stackrel{\circ}{n}\end{array}$ & \begin{tabular}{l}
$\mathcal{Y}$ \\
\multirow{j}{*}{} \\
$\infty$ \\
ల
\end{tabular} & 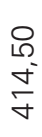 & 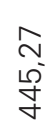 & $\begin{array}{l}\stackrel{P}{\circ} \\
0^{-} \\
\gamma\end{array}$ \\
\hline \multicolumn{2}{|c|}{ 完 } & & $\sum_{\underline{\underline{\Sigma}}}^{\bar{\Sigma}}$ & \begin{tabular}{l} 
O \\
\multirow{2}{*}{} \\
$\underset{v}{ }$
\end{tabular} & $\begin{array}{l}\infty \\
\text { ల } \\
\infty \\
\infty \\
\stackrel{\infty}{N}\end{array}$ & $\frac{\sigma}{\sigma}$ & 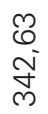 & $\begin{array}{l}\stackrel{8}{8} \\
\text { م } \\
\text { n }\end{array}$ & $\begin{array}{l}\hat{\infty} \\
\text { ब } \\
\text { ल }\end{array}$ & 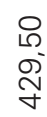 & 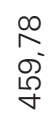 \\
\hline 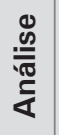 & & & $\begin{array}{l}\overline{\underline{\varepsilon}} \\
\underline{\underline{z}}\end{array}$ & $\begin{array}{l}\infty \\
\infty \\
\\
\curvearrowright\end{array}$ & $\begin{array}{l}\text { ন } \\
\text { ন্ }\end{array}$ & 市 & $\begin{array}{l}\text { Q } \\
\text { ల్ } \\
\text { లో }\end{array}$ & $\stackrel{0}{\hat{n}}$ & م) & \begin{tabular}{c}
$\mathscr{\infty}$ \\
$\stackrel{\sim}{\sim}$ \\
\multirow{\sigma}{*}{}
\end{tabular} & 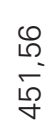 \\
\hline & $N$ & & छ & $\stackrel{\llcorner}{\sim}$ & $\underset{\sim}{\downarrow}$ & $\stackrel{m}{N}$ & $\approx$ & $\bar{N}$ & ㄱ. & $\stackrel{\sigma}{\square}$ & $\stackrel{\infty}{-}$ \\
\hline
\end{tabular}




\begin{tabular}{|c|c|c|c|c|c|c|c|c|c|c|c|}
\hline \multicolumn{2}{|c|}{$\begin{array}{l}\sum_{\text {D }} \\
\text { 是 }\end{array}$} & & $\underset{\underline{\underline{E}}}{\overline{\underline{\varepsilon}}}$ & $\begin{array}{l}\infty \\
\stackrel{\Sigma}{\Sigma} \\
\infty\end{array}$ & \begin{tabular}{l}
$\bar{N}$ \\
\multirow{+}{*}{} \\
$\infty$
\end{tabular} & $\begin{array}{l}\text { న } \\
\text { চ̊ }\end{array}$ & 孞 & $\begin{array}{l}8 \\
8 \\
\circ \\
8 \\
8\end{array}$ & $\begin{array}{l}8 \\
\text { ò } \\
\text { రె }\end{array}$ & \begin{tabular}{l}
8 \\
0 \\
Oे \\
0 \\
\hdashline
\end{tabular} & 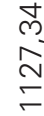 \\
\hline \multicolumn{2}{|c|}{$\begin{array}{l}\vec{D} \\
\text { }\end{array}$} & & $\begin{array}{l}\overline{\underline{\underline{\varepsilon}}} \\
\underline{\underline{\underline{z}}}\end{array}$ & $\begin{array}{l}\text { D } \\
0 \\
0 \\
0 \\
0\end{array}$ & $\begin{array}{l}8 \\
0 \\
\infty \\
0\end{array}$ & $\begin{array}{l}\infty \\
\stackrel{2}{ } \\
m^{-} \\
\stackrel{n}{n}\end{array}$ & $\begin{array}{l}\overline{0} \\
\text { 守 }\end{array}$ & $\begin{array}{l}\frac{1}{0} \\
\stackrel{\circ}{\circ} \\
\stackrel{0}{1}\end{array}$ & $\begin{array}{l}\infty \\
m \\
m \\
\stackrel{\infty}{\infty}\end{array}$ & $\begin{array}{l}\infty \\
0 \\
0 \\
0 \\
\infty\end{array}$ & $\begin{array}{l}10 \\
\circ \\
\circ \\
\infty \\
\infty\end{array}$ \\
\hline \multirow{5}{*}{ 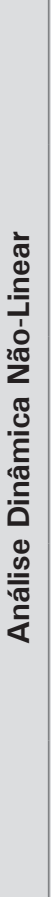 } & \multirow{5}{*}{ o } & $\begin{array}{l}\text { م } \\
\text { ๘ } \\
-\end{array}$ & 忘 & $\begin{array}{l}\text { मे } \\
\text { के } \\
\text { ठ }\end{array}$ & مُ & 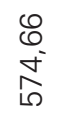 & $\begin{array}{l}\text { N } \\
\text { o } \\
\mathbb{O}^{\circ}\end{array}$ & 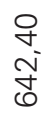 & $\begin{array}{l}L \\
\Omega \\
0 \\
0 \\
0\end{array}$ & $\begin{array}{l}\stackrel{5}{E} \\
\frac{1}{\pi}\end{array}$ & 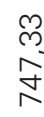 \\
\hline & & $\begin{array}{l}+ \\
\stackrel{+}{-}\end{array}$ & $\underset{\underline{\underline{\underline{E}}}}{\overline{\underline{\varepsilon}}}$ & $\begin{array}{l}\text { मे } \\
00^{\circ} \\
\text { ही }\end{array}$ & 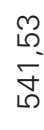 & 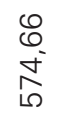 & $\begin{array}{l}\text { I } \\
\infty^{\circ} \\
\mathbb{O}^{2}\end{array}$ & 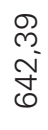 & $\begin{array}{l}\stackrel{2}{\Omega} \\
0 \\
0 \\
6\end{array}$ & $\frac{\mathscr{\Omega}}{\stackrel{-}{\pi}}$ & 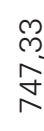 \\
\hline & & $\begin{array}{l}m \\
\text { o } \\
-\end{array}$ & $\begin{array}{l}\overline{\underline{\varepsilon}} \\
\underline{\underline{\underline{E}}}\end{array}$ & $\begin{array}{l}\text { m } \\
\circ \\
\circ \\
\circ \\
\circ\end{array}$ & 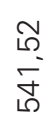 & 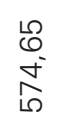 & $\begin{array}{l}\text { 尺े } \\
\text { ô } \\
0 \\
0\end{array}$ & 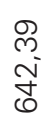 & $\begin{array}{l}\stackrel{L}{\Omega} \\
0 \\
0 \\
0\end{array}$ & $\frac{\mathscr{\Omega}}{\stackrel{-}{\pi}}$ & $\begin{array}{l}\stackrel{N}{~} \\
\stackrel{ }{ন}\end{array}$ \\
\hline & & $\begin{array}{l}\mathbf{N} \\
\underset{-}{-}\end{array}$ & $\begin{array}{l}\overline{\underline{\xi}} \\
\sum_{\underline{\underline{z}}}\end{array}$ & $\begin{array}{l}\text { న్ } \\
\delta^{\circ} \\
\delta^{2}\end{array}$ & م) & 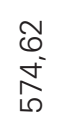 & $\begin{array}{l}\underset{N}{d} \\
\infty D^{\circ} \\
0\end{array}$ & 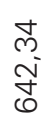 & $\begin{array}{l}\infty \\
\infty \\
0 \\
0 \\
0\end{array}$ & $\begin{array}{l}\infty \\
\infty \\
\frac{\infty}{\pi}\end{array}$ & $\begin{array}{l}\stackrel{\llcorner}{N} \\
\stackrel{ }{N}\end{array}$ \\
\hline & & $\begin{array}{l}\overline{0} \\
\text { o } \\
\text { ¿ }\end{array}$ & $\begin{array}{l}\overline{\underline{\varepsilon}} \\
\sum_{\underline{\underline{z}}}\end{array}$ & $\begin{array}{l}\text { న } \\
\varnothing^{\circ} \\
\overbrace{}^{\circ}\end{array}$ & $\begin{array}{l}\text { ले } \\
\text { - }\end{array}$ & 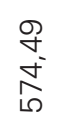 & 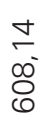 & 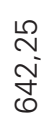 & $\begin{array}{l}\infty \\
0 \\
0 \\
0 \\
0\end{array}$ & $\begin{array}{l}\infty \\
\stackrel{\infty}{\leftarrow} \\
\frac{\pi}{\pi}\end{array}$ & $\begin{array}{l}\stackrel{N}{N} \\
\stackrel{N}{N}\end{array}$ \\
\hline \multicolumn{2}{|c|}{ एं } & & $\begin{array}{l}\overline{\underline{\varepsilon}} \\
\underline{\underline{\underline{E}}}\end{array}$ & $\begin{array}{l}\text { 寸 } \\
\text { ○े } \\
\text { ᄋ }\end{array}$ & $\begin{array}{l}\hat{0} \\
\text { N } \\
\text { దે }\end{array}$ & 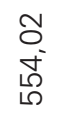 & 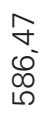 & $\begin{array}{l}\infty \\
m \\
\infty \\
\bar{\sigma}\end{array}$ & $\begin{array}{l}\stackrel{M}{N} \\
\underset{N}{0} \\
0\end{array}$ & $\begin{array}{l}\circ \\
0 \\
0 \\
00 \\
0\end{array}$ & $\begin{array}{l}\infty \\
0 \\
\circ \\
\stackrel{N}{N}\end{array}$ \\
\hline 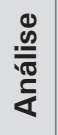 & 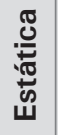 & & $\begin{array}{l}\overline{\underline{\xi}} \\
\text { 兰 }\end{array}$ & $\begin{array}{l}m \\
m \\
\stackrel{2}{\sigma} \\
\stackrel{+}{*}\end{array}$ & $\begin{array}{l}8 \\
0 \\
\hat{0} \\
\llcorner\end{array}$ & $\begin{array}{l}8 \\
8 \\
0 \\
0 \\
0 \\
\llcorner\end{array}$ & $\begin{array}{l}\infty \\
\circ \\
0^{\circ} \\
\mathbb{0}^{\circ} \\
10\end{array}$ & $\begin{array}{l}\circ \\
\text { ¿' } \\
\circ \\
\circ\end{array}$ & $\begin{array}{l}0 \\
\Theta \\
0 \\
0 \\
0 \\
0\end{array}$ & $\begin{array}{l}\stackrel{0}{N} \\
\stackrel{5}{0} \\
0\end{array}$ & $\begin{array}{l}\infty \\
m \\
\infty \\
\infty \\
0 \\
0\end{array}$ \\
\hline & $N$ & & $\underline{\xi}$ & $\stackrel{\sim}{\leftarrow}$ & $\stackrel{\oplus}{\sim}$ & $\stackrel{\llcorner}{\sim}$ & $\stackrel{\nabla}{\leftarrow}$ & $\stackrel{m}{\leftarrow}$ & $\stackrel{\sim}{\sim}$ & $\mp$ & $\stackrel{0}{\circ}$ \\
\hline
\end{tabular}




\begin{tabular}{|c|c|c|c|c|c|c|c|c|c|c|c|}
\hline \multicolumn{2}{|c|}{$\begin{array}{l}\text { 之 } \\
\text { 定 }\end{array}$} & & ${ }_{\underline{\underline{E}}}^{\overline{\underline{E}}}$ & $\begin{array}{l}m \\
\approx \\
\approx \\
\approx\end{array}$ & 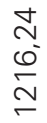 & $\begin{array}{l}0 \\
\infty \\
0 \\
0 \\
\mathbb{N} \\
\sim\end{array}$ & $\begin{array}{l}\hat{L} \\
\text { L̊ } \\
\text { ભి }\end{array}$ & $\begin{array}{l}0 \\
\text { n- } \\
0 \\
\text { ñ } \\
\end{array}$ & $\begin{array}{l}\mathscr{0} \\
\stackrel{5}{\circ} \\
\text { ले }\end{array}$ & 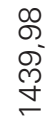 & 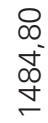 \\
\hline \multicolumn{2}{|c|}{ 虫 } & & $\begin{array}{l}\overline{\underline{E}} \\
\underline{\underline{\Sigma}}\end{array}$ & $\begin{array}{l}\hat{y} \\
\text { m. } \\
\dot{\sigma}\end{array}$ & $\begin{array}{l}\text { J } \\
\sigma^{\circ} \\
\text { రా }\end{array}$ & 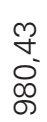 & $\begin{array}{l}\infty \\
\text { ஸ் } \\
\text { ஸ் }\end{array}$ & 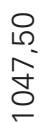 & 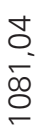 & \begin{tabular}{l} 
م \\
\multirow{\leftarrow}{\pm}{} \\
$E$
\end{tabular} & 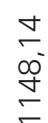 \\
\hline \multirow{5}{*}{ 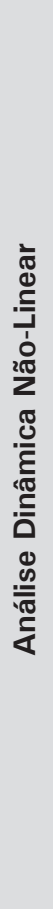 } & \multirow{5}{*}{ 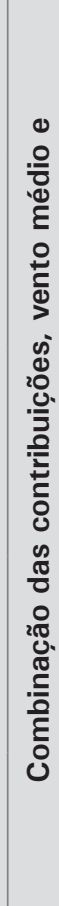 } & $\begin{array}{l}\text { م } \\
\sigma \\
\sigma\end{array}$ & $\begin{array}{l}\overline{\underline{\varepsilon}} \\
\underline{\underline{z}}\end{array}$ & 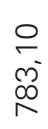 & $\begin{array}{l}\stackrel{m}{N} \\
\stackrel{\infty}{\infty}\end{array}$ & $\begin{array}{l}\infty \\
0 \\
L^{-1} \\
\infty \\
\infty\end{array}$ & $\begin{array}{l}\stackrel{+}{~} \\
\text { న } \\
\infty\end{array}$ & $\begin{array}{l}\text { f } \\
\text { ন } \\
\text { ه }\end{array}$ & 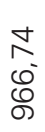 & $\begin{array}{l}\text { N } \\
\text { 广 } \\
8 \\
8\end{array}$ & $\begin{array}{l}\infty \\
\infty \\
\text { J } \\
\text { O }\end{array}$ \\
\hline & & $\begin{array}{l}\dot{\sigma} \\
\sigma \\
\sigma\end{array}$ & $\begin{array}{l}\overline{\underline{\varepsilon}} \\
\underline{\underline{z}}\end{array}$ & $\begin{array}{l}0 \\
\bar{N} \\
0 \\
\Gamma\end{array}$ & $\begin{array}{l}\stackrel{m}{N} \\
\frac{\sigma}{\infty}\end{array}$ & $\begin{array}{l}\infty \\
\complement \\
L^{-} \\
\infty \\
\infty\end{array}$ & $\begin{array}{l}\text { 寸 } \\
\text { న } \\
\infty\end{array}$ & \begin{tabular}{l}
0 \\
\multirow{+}{}{} \\
$\stackrel{N}{ }$
\end{tabular} & 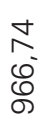 & 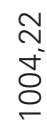 & $\begin{array}{l}\mathscr{\infty} \\
\infty \\
\check{\Xi} \\
\stackrel{\sigma}{-}\end{array}$ \\
\hline & & $\begin{array}{l}m \\
\sigma \\
\sigma\end{array}$ & $\begin{array}{l}\bar{\xi} \\
\underline{\underline{E}}\end{array}$ & 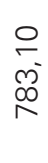 & $\begin{array}{l}\underset{N}{\infty} \\
\frac{\pi}{\infty}\end{array}$ & $\begin{array}{l}0 \\
0 \\
\llcorner \\
\infty \\
\infty\end{array}$ & \begin{tabular}{l} 
\} $\\
{\multirow{\infty}{*}{}} \\
{\infty}$ & 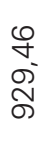 & $\begin{array}{l}m \\
10 \\
8 \\
8\end{array}$ & $\begin{array}{l}\text { N } \\
\text { ঠ } \\
\text { ○ }\end{array}$ & $\begin{array}{l}\infty \\
\infty \\
\bar{\Xi} \\
O\end{array}$ \\
\hline & & $\begin{array}{l}N \\
0 \\
\sigma\end{array}$ & $\begin{array}{l}\overline{\underline{\xi}} \\
\underline{\underline{z}}\end{array}$ & $\begin{array}{l}\text { mo } \\
\text { m. } \\
\text { o } \\
\text { o }\end{array}$ & $\begin{array}{l}\frac{0}{\sigma} \\
\frac{\sigma}{\infty}\end{array}$ & $\begin{array}{l}\widetilde{O} \\
\left\llcorner\rho^{-}\right. \\
\infty \\
\infty\end{array}$ & $\begin{array}{l}\text { ల } \\
\text { న } \\
\text { న }\end{array}$ & 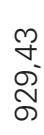 & $\begin{array}{l}N \\
\mathscr{C}^{-} \\
\stackrel{0}{0}\end{array}$ & $\begin{array}{l}\bar{v} \\
\text { ¿ } \\
\text { ○ }\end{array}$ & 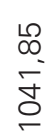 \\
\hline & & $\begin{array}{l}\overline{0} \\
\text { 음 } \\
\text { ㅁ }\end{array}$ & $\begin{array}{l}\overline{\underline{\xi}} \\
\underline{\underline{\underline{z}}}\end{array}$ & 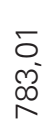 & $\frac{10}{\frac{1}{\sigma}}$ & $\begin{array}{l}\widetilde{\sigma} \\
\llcorner \\
\infty \\
\infty\end{array}$ & $\begin{array}{l}\text { లి } \\
\text { N } \\
\infty\end{array}$ & $\begin{array}{l}\text { m } \\
\text { D্ } \\
\text { চ }\end{array}$ & $\begin{array}{l}0 \\
\stackrel{0}{0} \\
0 \\
0\end{array}$ & 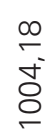 & $\begin{array}{l}\stackrel{0}{\infty} \\
\text { - } \\
\text { O- }\end{array}$ \\
\hline \multicolumn{2}{|c|}{ 定 } & & $\begin{array}{l}\overline{\underline{E}} \\
\underline{\underline{z}}\end{array}$ & $\underset{\stackrel{+}{\sim}}{\stackrel{+}{N}}$ & 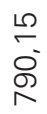 & $\begin{array}{l}\stackrel{\curvearrowright}{m} \\
\stackrel{\infty}{\infty} \\
\infty\end{array}$ & $\begin{array}{l}\text { న్ } \\
\text { ঠ் } \\
\varnothing\end{array}$ & 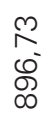 & $\begin{array}{l}\infty \\
0 \\
N \\
\text { ñ }\end{array}$ & 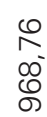 & $\begin{array}{l}\bar{\sigma} \\
\dot{\delta} \\
8\end{array}$ \\
\hline 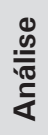 & 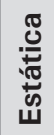 & & $\begin{array}{l}\bar{\xi} \\
\underline{\underline{\underline{E}}}\end{array}$ & $\frac{N}{\grave{N}}$ & $\frac{\infty}{\underset{j}{\infty}}$ & 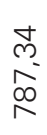 & 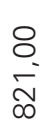 & $\begin{array}{l}+ \\
\stackrel{+}{\circ} \\
\infty\end{array}$ & $\begin{array}{l}\text { م) } \\
\text { D } \\
\infty \\
\infty\end{array}$ & $\begin{array}{r}\text { 尺 } \\
\underset{\sim}{\sim}\end{array}$ & $\begin{array}{l}\circ \\
\text { ס̊ } \\
\text { சீ }\end{array}$ \\
\hline & $N$ & & $\xi$ & $\sigma$ & $\infty$ & 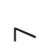 & 0 & ما & $\checkmark$ & $m$ & $N$ \\
\hline
\end{tabular}
\end{tabular}




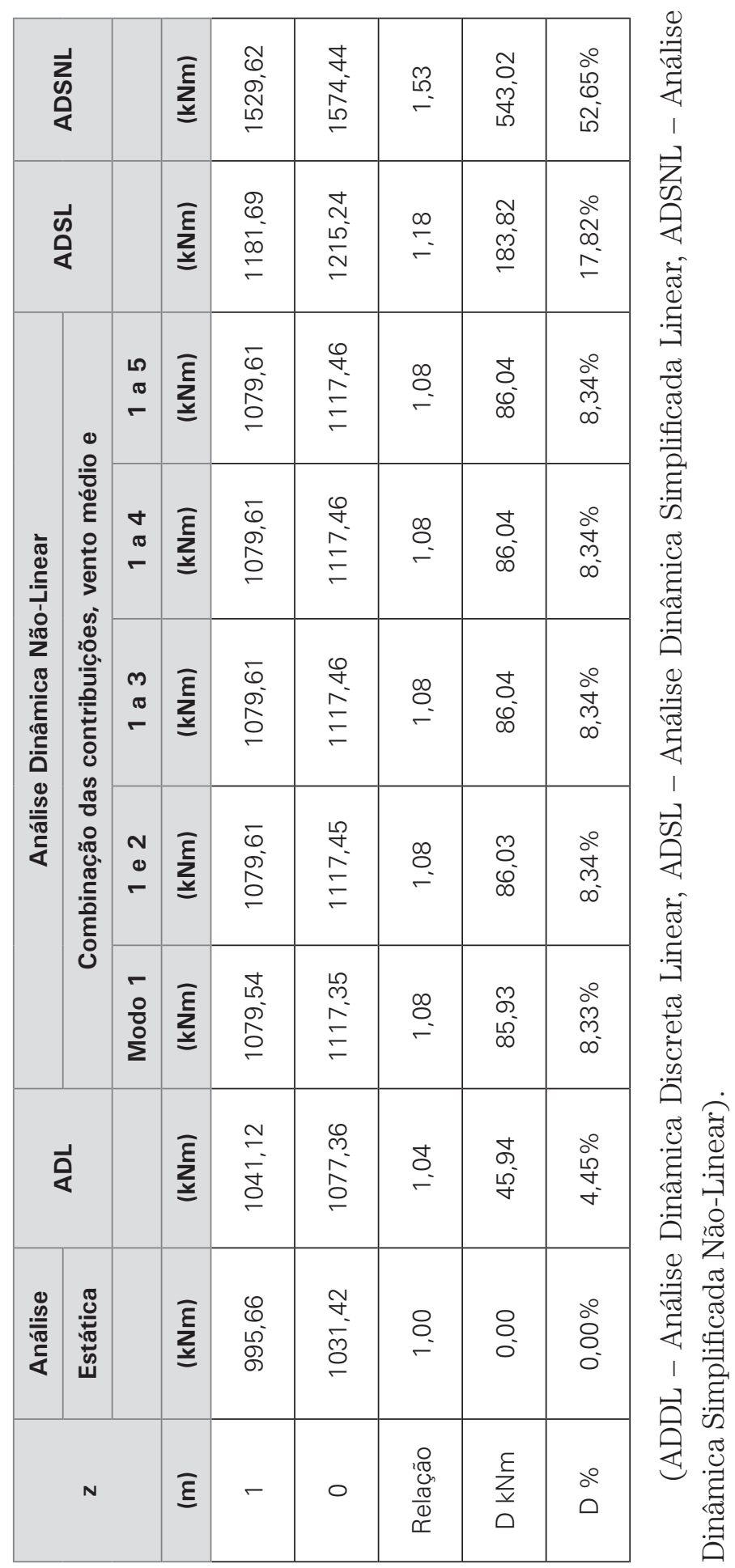


No gráfico da Figura 7.25 podem ser encontrados os resultados obtidos para a ação do vento sobre a estrutura. No traço mais inferior estão os valores do momento para a análise estática. Os traçados superiores subsequentes correspondem aos valores das análises dinâmicas discretas. Logo acima se encontram as curvas das análises dinâmicas simplificadas, linear e não-linear.

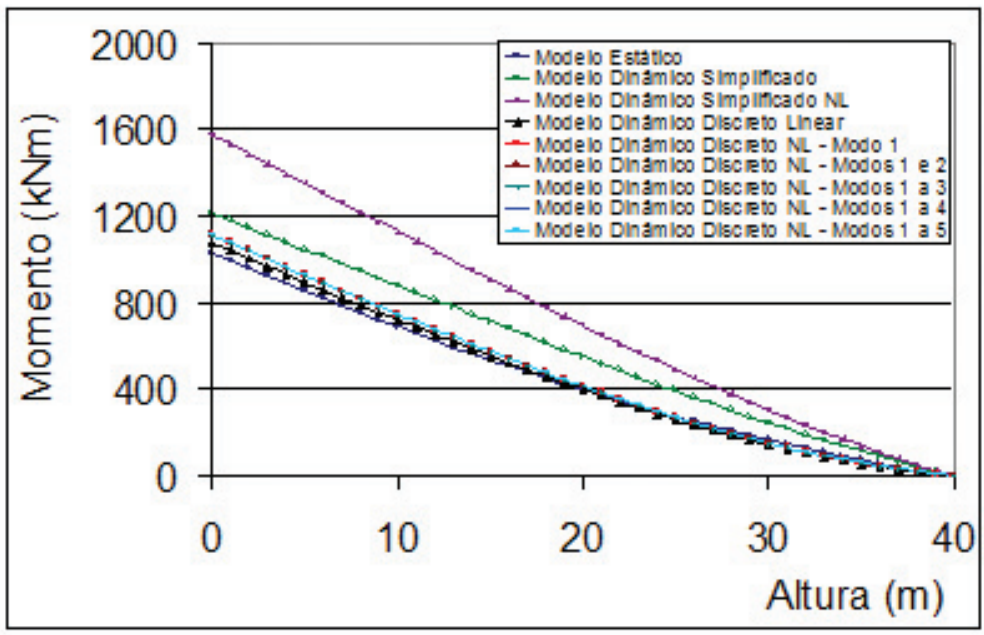

Figura 7.25 - Ação do vento na estrutura $4(\zeta=0,015)$.

As formas modais da NBR 6123/88, do Método dos Elementos Finitos e do Método proposto constam na Figura 7.26(a).

Uma melhor aproximação à forma modal não-linear é conseguida substituindo-se o expoente da expressão (6.6) por 1,77; tendo em vista que a curva definida com esse novo expoente oferece uma menor diferença entre seus pontos e os da curva da forma modal não-linear, como pode ser observado na Figura 7.26(b)

O expoente sugerido anteriormente distancia-se 34\% do valor do recomendado pela NBR 6123/88. 


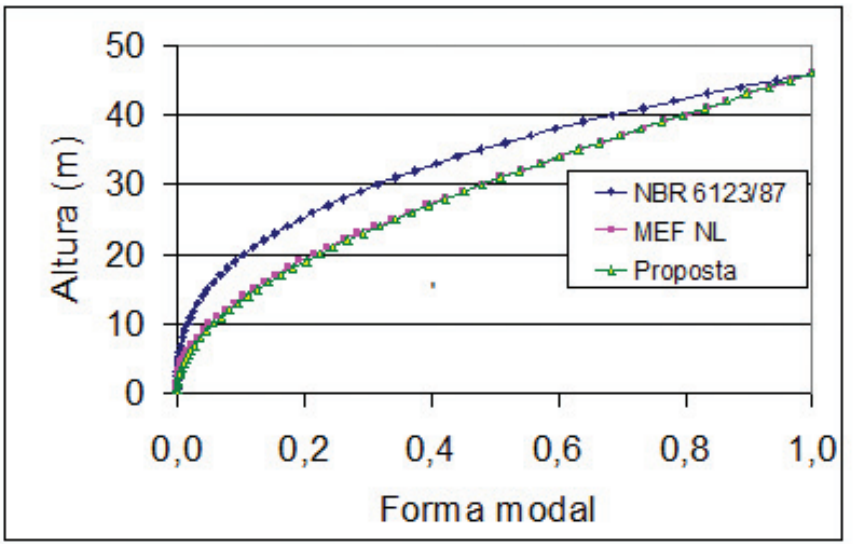

(a) expoente da forma: $\gamma=2,7$

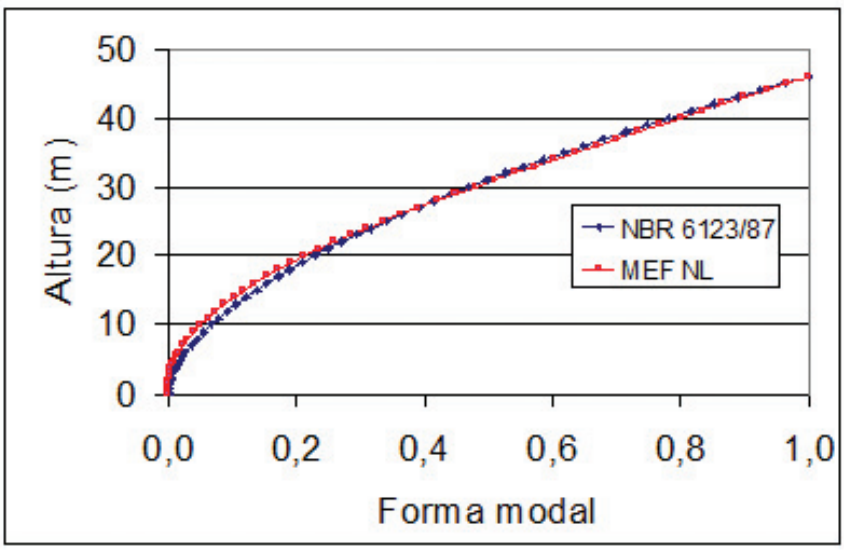

(b) expoente da forma modal: $\gamma=1,77$

Figura 7.26 - Estrutura 4 - comparativo das formas modais: (a) usada na análise, (b) sugerida.

Ter-se-ia, entretanto, um panorama mais crítico que o anterior caso se adotasse uma razão de amortecimento crítico de 0,01. Nesse caso, o modelo dinâmico simplificado linear seria o correspondente à forma modal com expoente de 1,7 na Eq. (6.6), e essa opção levaria o período de oscilação do primeiro modo a ser calculado usando 1,5\% da altura da estrutura, o que forneceria a frequência fundamental de $0,69 \mathrm{~Hz}$. Nessa situação as flutuações do primeiro modo responderiam por 70\% da resposta dinâmica da estrutura, quando superposta à contribuição do vento médio. 
Seguindo-se com a análise para essa condição, a diferença produzida entre o valor do momento fletor máximo, do modelo discreto, do primeiro ao quinto modo, com as considerações não-lineares, tanto material quanto geométrica, chegariam a superar em 1,41 a análise das forças estáticas. E seria superior em 4,28\% à análise discreta linear ao computar-se apenas o primeiro modo. Os resultados podem ser vistos na Tabela 7.22, na Tabela 7.23 e no gráfico da Figura 7.27.

Tabela 7.21 - Estrutura 4: momentos da análise discreta nãolinear $(\zeta=0,01)$.

\begin{tabular}{|c|c|c|c|c|c|c|}
\hline \multirow{2}{*}{$\mathbf{z}$} & \multirow{2}{*}{$\begin{array}{l}\text { Vento } \\
\text { Médio }\end{array}$} & \multicolumn{5}{|c|}{ Flutuações } \\
\hline & & Modo1 & Modo 2 & Modo 3 & Modo 4 & Modo 5 \\
\hline (m) & (kNm) & (kNm) & (kNm) & (kNm) & (kNm) & (kNm) \\
\hline 40 & 0,00 & 0,00 & 0,00 & 0,00 & 0,00 & 0,00 \\
\hline 39 & 6,23 & 4,13 & 0,48 & 0,82 & 0,45 & 0,41 \\
\hline 38 & 12,78 & 10,08 & 1,14 & 1,92 & 1,04 & 0,93 \\
\hline 37 & 19,65 & 17,79 & 1,97 & 3,23 & 1,69 & 1,46 \\
\hline 36 & 26,84 & 27,18 & 2,93 & 4,66 & 2,34 & 1,92 \\
\hline 35 & 34,35 & 38,20 & 4,00 & 6,14 & 2,94 & 2,26 \\
\hline 34 & 42,16 & 50,79 & 5,15 & 7,60 & 3,42 & 2,43 \\
\hline 33 & 50,28 & 64,88 & 6,35 & 8,96 & 3,76 & 2,40 \\
\hline 32 & 58,71 & 80,41 & 7,59 & 10,16 & 3,91 & 2,16 \\
\hline 31 & 67,43 & 97,32 & 8,83 & 11,15 & 3,87 & 1,74 \\
\hline 30 & 76,44 & 115,55 & 10,05 & 11,89 & 3,62 & 1,17 \\
\hline
\end{tabular}




\begin{tabular}{|c|c|c|c|c|c|c|}
\hline \multirow{2}{*}{$\mathbf{z}$} & \multirow{2}{*}{$\begin{array}{l}\text { Vento } \\
\text { Médio }\end{array}$} & \multicolumn{5}{|c|}{ Flutuações } \\
\hline & & Modo1 & Modo 2 & Modo 3 & Modo 4 & Modo 5 \\
\hline (m) & (kNm) & $(\mathrm{kNm})$ & (kNm) & (kNm) & (kNm) & (kNm) \\
\hline 29 & 85,74 & 135,04 & 11,24 & 12,34 & 3,19 & 0,49 \\
\hline 28 & 95,33 & 155,72 & 12,35 & 12,48 & 2,58 & 0,22 \\
\hline 27 & 105,19 & 177,55 & 13,39 & 12,29 & 1,83 & 0,91 \\
\hline 26 & 115,33 & 200,44 & 14,33 & 11,77 & 0,99 & 1,51 \\
\hline 25 & 125,74 & 224,36 & 15,15 & 10,93 & 0,10 & 1,96 \\
\hline 24 & 136,42 & 249,24 & 15,83 & 9,79 & 0,79 & 2,23 \\
\hline 23 & 147,35 & 275,02 & 16,37 & 8,38 & 1,63 & 2,28 \\
\hline 22 & 158,55 & 301,64 & 16,74 & 6,73 & 2,37 & 2,12 \\
\hline 21 & 169,99 & 329,06 & 16,95 & 4,90 & 2,96 & 1,75 \\
\hline 20 & 181,68 & 357,22 & 16,98 & 2,93 & 3,38 & 1,22 \\
\hline 19 & 193,60 & 386,07 & 16,83 & 0,88 & 3,59 & 0,57 \\
\hline 18 & 205,76 & 415,55 & 16,49 & 1,20 & 3,58 & 0,13 \\
\hline 17 & 218,15 & 445,61 & 15,97 & 3,24 & 3,36 & 0,83 \\
\hline 16 & 230,76 & 476,22 & 15,26 & 5,20 & 2,94 & 1,45 \\
\hline 15 & 243,59 & 507,31 & 14,36 & 7,00 & 2,33 & 1,94 \\
\hline
\end{tabular}




\begin{tabular}{|c|c|c|c|c|c|c|}
\hline \multirow{2}{*}{ z } & \multirow{2}{*}{$\begin{array}{l}\text { Vento } \\
\text { Médio }\end{array}$} & \multicolumn{5}{|c|}{ Flutuações } \\
\hline & & Modo1 & Modo 2 & Modo 3 & Modo 4 & Modo 5 \\
\hline (m) & (kNm) & (kNm) & (kNm) & (kNm) & (kNm) & (kNm) \\
\hline 14 & 256,63 & 538,85 & 13,28 & 8,61 & 1,57 & 2,25 \\
\hline 13 & 269,87 & 570,80 & 12,02 & 9,97 & 0,70 & 2,35 \\
\hline 12 & 283,31 & 603,14 & 10,59 & 11,05 & 0,23 & 2,24 \\
\hline 11 & 296,94 & 635,85 & 8,98 & 11,78 & 1,18 & 1,89 \\
\hline 10 & 310,75 & 668,90 & 7,19 & 12,14 & 2,08 & 1,36 \\
\hline 9 & 324,75 & 702,25 & 5,24 & 12,10 & 2,90 & 0,68 \\
\hline 8 & 338,92 & 735,88 & 3,14 & 11,66 & 3,57 & 0,09 \\
\hline 7 & 353,25 & 769,76 & 0,89 & 10,81 & 4,05 & 0,87 \\
\hline 6 & 367,75 & 803,85 & 1,48 & 9,57 & 4,31 & 1,59 \\
\hline 5 & 382,39 & 838,13 & 3,97 & 7,95 & 4,32 & 2,18 \\
\hline 4 & 397,18 & 872,57 & 6,57 & 5,98 & 4,08 & 2,58 \\
\hline 3 & 412,09 & 907,13 & 9,24 & 3,70 & 3,59 & 2,75 \\
\hline 2 & 427,07 & 941,79 & 11,99 & 1,15 & 2,86 & 2,67 \\
\hline 1 & 442,11 & 976,53 & 14,79 & 1,63 & 1,91 & 2,32 \\
\hline 0 & 457,20 & 1011,33 & 17,64 & 4,59 & 0,77 & 1,72 \\
\hline
\end{tabular}




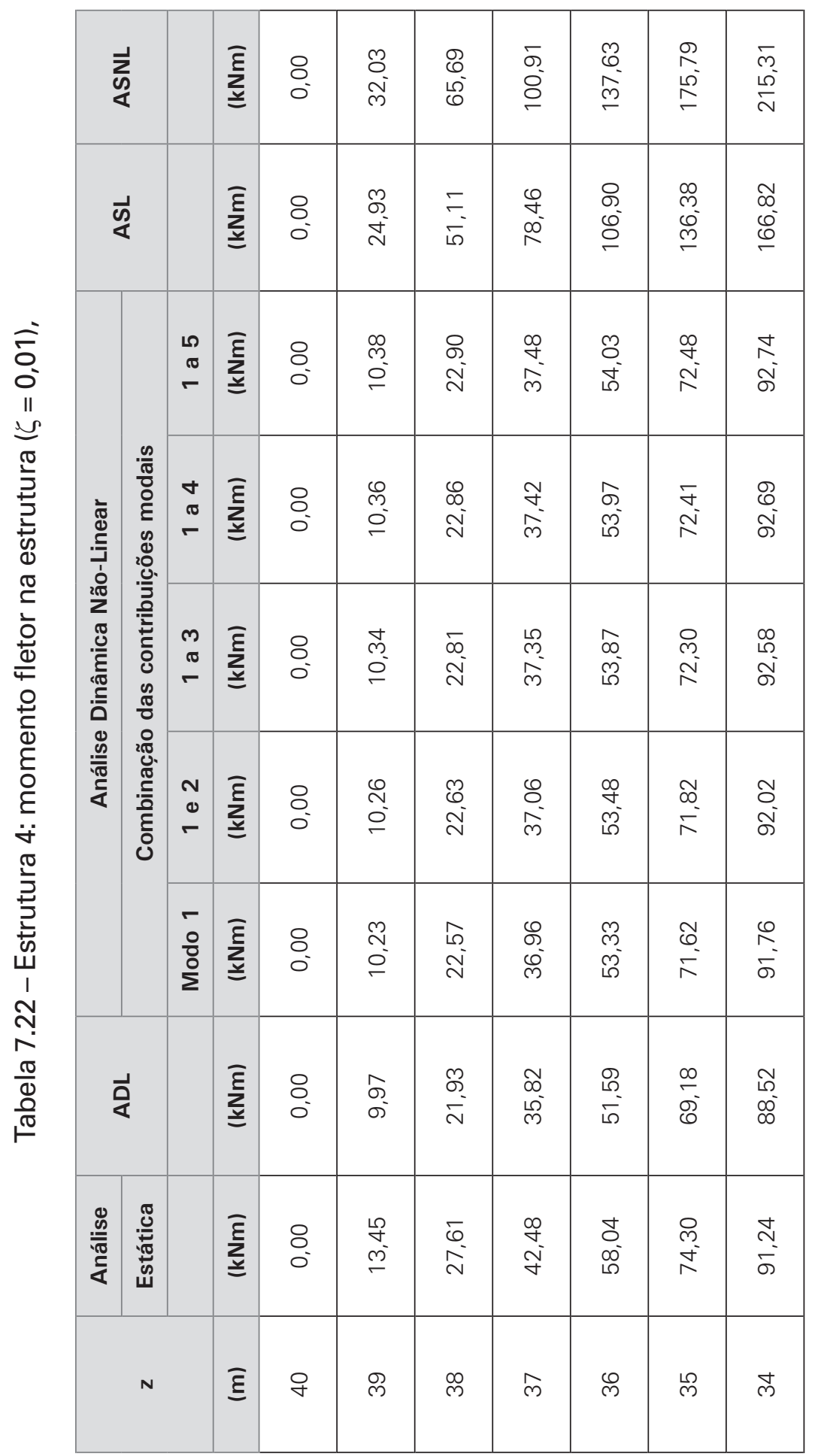




\begin{tabular}{|c|c|c|c|c|c|c|c|c|c|c|c|}
\hline \multicolumn{2}{|c|}{ 定 } & & $\sum_{\underline{\underline{E}}}^{\bar{E}}$ & \begin{tabular}{l}
$\checkmark$ \\
\multirow{\circ}{\circ}{} \\
$\stackrel{D}{N}$
\end{tabular} & 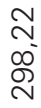 & 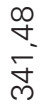 & $\begin{array}{l}\mathscr{Q} \\
\infty \\
\infty \\
\infty \\
m\end{array}$ & $\bar{m}$ & $\stackrel{\hat{N}}{\stackrel{\hat{\gamma}}{\sigma}}$ & $\begin{array}{l}\infty \\
\stackrel{\infty}{\sim} \\
\stackrel{\sim}{\sim}\end{array}$ & $\begin{array}{l}\text { g } \\
\text { m } \\
\stackrel{0}{0}\end{array}$ \\
\hline \multicolumn{2}{|c|}{ ङ } & & $\begin{array}{l}\overline{\underline{\xi}} \\
\text { 兰 }\end{array}$ & $\begin{array}{l}0 \\
\stackrel{0}{\infty} \\
\stackrel{\circ}{\sigma}\end{array}$ & $\begin{array}{l}\text { N } \\
\text { ల్ } \\
\text { N }\end{array}$ & 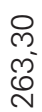 & $\begin{array}{l}\text { ब } \\
\text { ச } \\
\text { న }\end{array}$ & 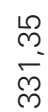 & $\begin{array}{l}m \\
m \\
0 \\
\mathbb{E} \\
m\end{array}$ & $\begin{array}{l}\text { ' } \\
\infty \\
\text { ó }\end{array}$ & $\begin{array}{l}\hat{O} \\
\hat{m} \\
\text { Oे }\end{array}$ \\
\hline \multirow{5}{*}{ 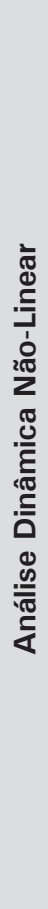 } & \multirow{5}{*}{ 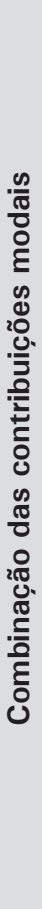 } & $\begin{array}{l}10 \\
\sigma \\
\sigma\end{array}$ & $\sum_{\underline{\underline{E}}}^{\bar{\xi}}$ & $\begin{array}{l}0 \\
\stackrel{ \pm}{E} \\
=\end{array}$ & \begin{tabular}{l}
\multirow{\sigma}{*}{} \\
$\infty$ \\
$\stackrel{-}{-}$
\end{tabular} & $\begin{array}{l}\bar{\infty} \\
\text { లె }^{-}\end{array}$ & 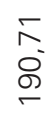 & $\frac{m}{\frac{m}{\sigma}}$ & 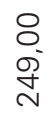 & $\begin{array}{l}0 \\
\stackrel{2}{ } \\
0 \\
\stackrel{0}{N}\end{array}$ & $\begin{array}{l}\stackrel{\infty}{\infty} \\
\stackrel{\sim}{m}\end{array}$ \\
\hline & & $\begin{array}{l}\text { + } \\
\text { ब }\end{array}$ & $\begin{array}{l}\bar{\xi} \\
\sum_{\underline{E}}\end{array}$ & $\begin{array}{l}N \\
\pm \\
\stackrel{E}{E}\end{array}$ & $\begin{array}{l}\text { \& } \\
\infty \\
\stackrel{-}{m}\end{array}$ & 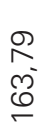 & $\begin{array}{l}\overline{1} \\
\tilde{0} \\
-\end{array}$ & $\frac{m}{\frac{m}{\sigma}}$ & 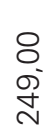 & $\begin{array}{l}0 \\
\stackrel{N}{0} \\
\stackrel{0}{N} \\
\stackrel{0}{0}\end{array}$ & $\begin{array}{l}\stackrel{\llcorner}{\infty} \\
\stackrel{\infty}{N} \\
\stackrel{m}{n}\end{array}$ \\
\hline & & $\begin{array}{l}m \\
\sigma \\
\sigma\end{array}$ & $\begin{array}{l}\bar{\xi} \\
\underline{\underline{E}}\end{array}$ & $\begin{array}{l}\bar{\sigma} \\
\pm \\
\mp\end{array}$ & 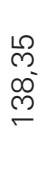 & 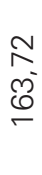 & $\begin{array}{l}\text { D } \\
0 \\
8 \\
0\end{array}$ & $\begin{array}{l}\text { g. } \\
\text { D. } \\
\bar{N}\end{array}$ & $\begin{array}{l}\infty \\
\stackrel{\infty}{ } \\
\stackrel{\infty}{+} \\
\stackrel{+}{\sim}\end{array}$ & $\begin{array}{l}\stackrel{L}{N} \\
\stackrel{0}{0} \\
\stackrel{0}{N}\end{array}$ & $\begin{array}{l}+\infty \\
\stackrel{+}{N} \\
\stackrel{m}{2}\end{array}$ \\
\hline & & $\begin{array}{l}N \\
0 \\
r\end{array}$ & $\begin{array}{l}\bar{\xi} \\
\bar{\sum}\end{array}$ & $\begin{array}{l}\overline{0} \\
\pm \\
\mp\end{array}$ & $\frac{N}{N}$ & $\begin{array}{l}8 \\
\text { §ె } \\
6\end{array}$ & $\begin{array}{l}\text { Lீ } \\
\circ \\
8 \\
8\end{array}$ & 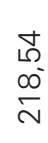 & $\begin{array}{l}\stackrel{\infty}{+} \\
\stackrel{\infty}{+} \\
\sim\end{array}$ & $\begin{array}{l}m \\
\infty \\
\text { D' } \\
\text { N }\end{array}$ & $\begin{array}{c}\stackrel{ }{\infty} \\
\text { N } \\
\text { n }\end{array}$ \\
\hline & & $\begin{array}{l}\overline{0} \\
\text { 음 } \\
\Sigma\end{array}$ & $\underline{\underline{\varepsilon}}_{\underline{\underline{\varepsilon}}}$ & $\begin{array}{l}\stackrel{P}{2} \\
\stackrel{m}{\mp}\end{array}$ & $\begin{array}{l}\hat{m} \\
\hat{m}\end{array}$ & 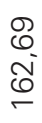 & $\begin{array}{l}\text { ర్ } \\
\text { న } \\
\infty\end{array}$ & $\begin{array}{l}\hat{0} \\
\infty \\
\bar{N} \\
\bar{\sigma}\end{array}$ & $\begin{array}{l}\text { ৪ } \\
\text { o- } \\
\stackrel{+}{\sim}\end{array}$ & $\begin{array}{l}m \\
m \\
\stackrel{m}{N} \\
\stackrel{n}{2}\end{array}$ & $\frac{\text { R. }}{\text { - }}$ \\
\hline \multicolumn{2}{|c|}{ 只 } & & ${ }_{\underline{\underline{\xi}}}^{\overline{\underline{E}}}$ & $\begin{array}{l}\hat{L} \\
\text { g̊ } \\
\circ\end{array}$ & $\stackrel{\sim}{\sim}$ & $\begin{array}{l}\llcorner \\
\omega^{\circ} \\
\curvearrowleft\end{array}$ & $\begin{array}{l}\stackrel{\circ}{\infty} \\
\stackrel{2}{\infty} \\
\infty\end{array}$ & 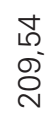 & $\stackrel{\infty}{\infty} \underset{\infty}{\infty}$ & $\begin{array}{l}\mathscr{0} \\
\infty 0^{-} \\
\stackrel{0}{N}\end{array}$ & $\begin{array}{l}\text { ণ } \\
\text { ণ } \\
\stackrel{\sim}{\sim}\end{array}$ \\
\hline 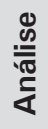 & 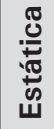 & & $\begin{array}{l}\overline{\underline{\varepsilon}} \\
\underline{\underline{\underline{z}}}\end{array}$ & $\begin{array}{l}0 \\
\infty \\
\infty \\
0\end{array}$ & $\stackrel{\infty}{\stackrel{\sim}{N}}$ & $\begin{array}{l}0 \\
\stackrel{0}{0} \\
\stackrel{+}{\tau}\end{array}$ & $\begin{array}{l}\bar{\infty} \\
\sigma \\
0 \\
0\end{array}$ & $\begin{array}{l}\mathcal{V} \\
0^{-} \\
\infty\end{array}$ & $\begin{array}{l}\text { o } \\
\text { ㅇ. } \\
\text { ㅇ }\end{array}$ & $\begin{array}{l}\bar{N} \\
\infty \\
\stackrel{N}{N}\end{array}$ & $\begin{array}{l}\hat{n} \\
0 \\
0 \\
\stackrel{0}{N}\end{array}$ \\
\hline & $N$ & & $\underline{\xi}$ & $m$ & m & m & $m$ & $\stackrel{\Omega}{\sim}$ & $\stackrel{\infty}{N}$ & $\widehat{N}$ & $\stackrel{\bullet}{\sim}$ \\
\hline
\end{tabular}




\begin{tabular}{|c|c|c|c|c|c|c|c|c|c|c|c|}
\hline \multicolumn{2}{|c|}{ 岕 } & & $\begin{array}{l}\bar{\xi} \\
\underline{\underline{E}}\end{array}$ & $\begin{array}{l}\text { ம } \\
\text { N } \\
\widetilde{0}\end{array}$ & \begin{tabular}{l} 
D \\
N \\
\multirow{0}{0}{}
\end{tabular} & $\begin{array}{l}\bar{N} \\
\stackrel{N}{N}\end{array}$ & $\begin{array}{l}\text { O } \\
\text { N } \\
\text { N }\end{array}$ & 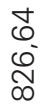 & $\begin{array}{l}\stackrel{m}{v} \\
\text { D } \\
\infty\end{array}$ & $\begin{array}{l}\infty \\
\text { m} \\
\text { N } \\
\text { ñ }\end{array}$ & $\begin{array}{l}\text { ¿ } \\
0 \\
0 \\
0 \\
0\end{array}$ \\
\hline & p & & $\bar{\sum}_{\underline{\underline{E}}}^{\bar{\varepsilon}}$ & 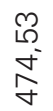 & $\frac{\underbrace{+}}{\stackrel{5}{-}}$ & 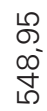 & $\begin{array}{l}N \\
0 \\
0 \\
0\end{array}$ & 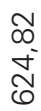 & $\begin{array}{l}\text { N } \\
\text { ָ̃ } \\
\mathbb{0}\end{array}$ & $\begin{array}{l}\infty \\
\infty \\
\infty \\
\infty \\
\Gamma\end{array}$ & $\begin{array}{l}\infty \\
\stackrel{\infty}{\circ} \\
\stackrel{+}{N}\end{array}$ \\
\hline \multirow{5}{*}{ 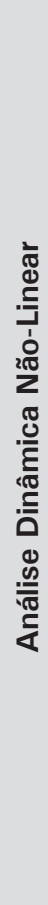 } & \multirow{5}{*}{ 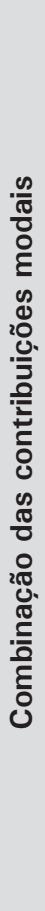 } & $\begin{array}{l}\text { م } \\
\sigma \\
\sigma\end{array}$ & $\sum_{\underline{\underline{E}}}^{\bar{\xi}}$ & $\begin{array}{c}\stackrel{N}{N} \\
\text { ले }\end{array}$ & $\begin{array}{l}\stackrel{\infty}{\infty} \\
\text { - } \\
\text { }\end{array}$ & $\begin{array}{l}\dot{\sigma} \\
\infty \\
\infty \\
\dot{\sigma}\end{array}$ & $\begin{array}{l}\infty \\
m \\
\Gamma^{\infty} \\
\stackrel{\gamma}{q}\end{array}$ & 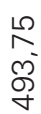 & $\begin{array}{l}\check{E} \\
\tilde{m} \\
\tilde{n}\end{array}$ & 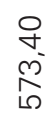 & $\frac{1}{\sigma}$ \\
\hline & & $\begin{array}{l}\dot{\sigma} \\
\sigma \\
\sigma\end{array}$ & $\begin{array}{l}\bar{\varepsilon} \\
\underline{\underline{z}}\end{array}$ & $\begin{array}{l}\overline{7} \\
0 \\
\text { o' }\end{array}$ & $\begin{array}{l}\text { D } \\
\bar{\infty} \\
\bar{m}\end{array}$ & $\begin{array}{l}m \\
0 \\
\infty \\
\sigma \\
\sigma\end{array}$ & $\begin{array}{l}\hat{n} \\
\stackrel{\rho}{\rho} \\
\stackrel{\sigma}{\sigma}\end{array}$ & 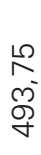 & Г̃ & 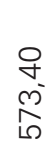 & $\begin{array}{l}\stackrel{L}{L} \\
\underset{\sim}{+}\end{array}$ \\
\hline & & $\begin{array}{l}m \\
\sigma \\
\sigma\end{array}$ & $\sum_{\underline{\underline{E}}}^{\bar{\xi}}$ & $\begin{array}{l}\bar{r} \\
0 \\
\tilde{m}\end{array}$ & $\frac{\text { g }}{N}$ & 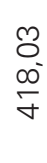 & $\begin{array}{l}0 \\
m \\
\mathcal{L}^{\circ} \\
\text { مै }\end{array}$ & $\begin{array}{l}\text { N } \\
\text { ণ্ } \\
\stackrel{+}{+}\end{array}$ & $\begin{array}{l}\text { ' } \\
\text { ले } \\
\text { లె }\end{array}$ & $\begin{array}{l}\infty \\
m \\
\stackrel{m}{n} \\
\end{array}$ & 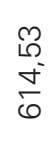 \\
\hline & & $\begin{array}{l}N \\
0 \\
\sigma\end{array}$ & $\begin{array}{l}\bar{\varepsilon} \\
\underline{\underline{E}}\end{array}$ & 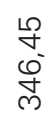 & 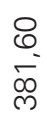 & $\begin{array}{l}\text { O } \\
\text { N- } \\
\bar{\sigma}\end{array}$ & $\begin{array}{l}\stackrel{9}{\sim} \\
\stackrel{\sim}{\sim} \\
\sim\end{array}$ & 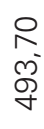 & $\begin{array}{l}\infty \\
0 \\
\text { లె } \\
\text { లె }\end{array}$ & $\begin{array}{l}\infty \\
\stackrel{\infty}{n} \\
\end{array}$ & $\frac{n}{\sigma}$ \\
\hline & & $\begin{array}{l}\overline{0} \\
\frac{0}{0} \\
\dot{0}\end{array}$ & $\sum_{\underline{\underline{z}}}^{\bar{\varepsilon}}$ & $\begin{array}{l}\text { J } \\
\text { ळ } \\
\text { లे }\end{array}$ & $\begin{array}{l}\circ \\
\bar{\infty} \\
\text { ळ }\end{array}$ & $\frac{\stackrel{f}{+}}{\frac{\sigma}{\sigma}}$ & \begin{tabular}{l}
$\infty$ \\
$\infty$ \\
\multirow{\leftarrow}{*}{} \\
$\stackrel{+}{+}$
\end{tabular} & 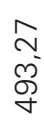 & 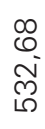 & 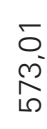 & $\begin{array}{l}\bar{N} \\
\dot{\sigma} \\
\bar{\sigma}\end{array}$ \\
\hline \multicolumn{2}{|c|}{ 完 } & & $\sum_{\underline{E}}^{\bar{E}}$ & $\begin{array}{l}\text { 品 } \\
\text { m }\end{array}$ & $\begin{array}{l}\llcorner \\
\llcorner \\
\llcorner \\
0 \\
0\end{array}$ & $\begin{array}{l}\text { ᄋ } \\
\text { O̊ } \\
\text { ᄋ }\end{array}$ & 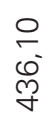 & 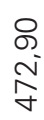 & 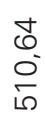 & 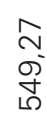 & $\begin{array}{l}m \\
\infty \\
\infty \\
\infty \\
\infty\end{array}$ \\
\hline 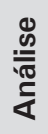 & 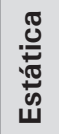 & & $\underset{\underline{\underline{\varepsilon}}}{\underline{\underline{\underline{E}}}}$ & \begin{tabular}{l}
$\infty$ \\
$\infty$ \\
$\stackrel{\infty}{N}$ \\
\multirow{N}{*}{}
\end{tabular} & $\begin{array}{l}\bar{\gamma} \\
\text { ন } \\
\text { N }\end{array}$ & 怘 & 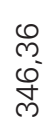 & $\stackrel{0}{\stackrel{n}{N}}$ & م) & $\begin{array}{l}\mathscr{N} \\
\underset{\sim}{\sim}\end{array}$ & L \\
\hline & $\mathbf{N}$ & & $\bar{\xi}$ & $\sim$ & $\stackrel{\sim}{\sim}$ & $\stackrel{N}{N}$ & $\approx$ & $\bar{v}$ & $\stackrel{\sim}{N}$ & $\stackrel{\Omega}{\square}$ & $\stackrel{\infty}{\sim}$ \\
\hline
\end{tabular}




\begin{tabular}{|c|c|c|c|c|c|c|c|c|c|c|c|}
\hline \multicolumn{2}{|c|}{ 离 } & & $\begin{array}{l}\overline{\underline{\xi}} \\
\underline{\underline{z}}\end{array}$ & 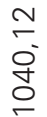 & 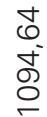 & 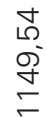 & 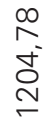 & $\begin{array}{l}m \\
\text { m } \\
0 \\
\stackrel{0}{N} \\
\text { - }\end{array}$ & $\frac{n}{\frac{0}{\sigma}}$ & $\begin{array}{r}\bar{N} \\
\stackrel{n}{N}\end{array}$ & $\begin{array}{l}\stackrel{\bigcirc}{\llcorner} \\
\infty^{-} \\
\stackrel{\sim}{\sim} \\
\leftarrow\end{array}$ \\
\hline $\bar{u}$ & & & $\begin{array}{l}\bar{\xi} \\
\underline{\underline{z}}\end{array}$ & $\begin{array}{l}\infty \\
\infty \\
\infty \\
\curvearrowright \\
N\end{array}$ & $\frac{N}{\sigma}$ & $\begin{array}{l}\mathcal{N} \\
\infty \\
\stackrel{0}{ } \\
\infty\end{array}$ & $\begin{array}{l}\bar{N} \\
\infty \\
\infty \\
\infty\end{array}$ & $\begin{array}{l}\text { n } \\
\text { ñ }\end{array}$ & $\frac{N}{N}$ & $\frac{\bar{\sigma}}{\tilde{\sigma}}$ & مิ \\
\hline \multirow{5}{*}{ 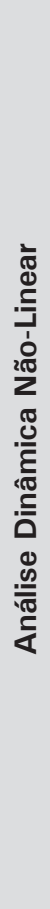 } & \multirow{5}{*}{ 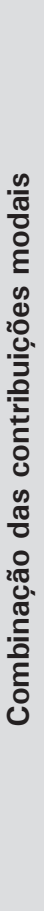 } & $\begin{array}{l}0 \\
\text { గ } \\
\sigma\end{array}$ & ${ }_{\underline{\underline{E}}}^{\bar{E}}$ & 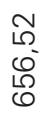 & 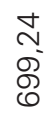 & \begin{tabular}{l}
$\hat{0}$ \\
\multirow{N}{N}{} \\
N
\end{tabular} & $\begin{array}{l}\stackrel{\omega}{\wedge} \\
0^{\circ} \\
\infty\end{array}$ & $\begin{array}{l}\underset{N}{J} \\
\bar{\infty}\end{array}$ & $\begin{array}{l}0 \\
0 \\
0 \\
\infty\end{array}$ & 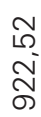 & $\begin{array}{l}\llcorner 0 \\
\infty \\
\infty \\
\mathscr{0}\end{array}$ \\
\hline & & $\begin{array}{l}+ \\
\sigma \\
\sigma\end{array}$ & $\begin{array}{l}\overline{\underline{\xi}} \\
\underline{\underline{\sum}}\end{array}$ & $\begin{array}{l}\text { న్ } \\
0 \\
0 \\
0 \\
0\end{array}$ & 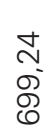 & \begin{tabular}{l}
$\hat{0}$ \\
\multirow{N}{*}{} \\
N
\end{tabular} & $\begin{array}{l}\stackrel{\llcorner}{\curvearrowright} \\
0^{-} \\
\infty\end{array}$ & \begin{tabular}{l}
$m$ \\
\multirow{\infty}{m}{} \\
$\infty$
\end{tabular} & $\begin{array}{l}\text { の } \\
0 \\
\infty\end{array}$ & \begin{tabular}{l}
$\bar{\Sigma}$ \\
\multirow{N}{\sigma}{} \\
ñ
\end{tabular} & $\begin{array}{l}\infty \\
\infty \\
\infty \\
\infty \\
\varnothing\end{array}$ \\
\hline & & $\begin{array}{l}m \\
\sigma \\
\sigma\end{array}$ & $\begin{array}{l}\bar{E} \\
\text { 经 }\end{array}$ & $\begin{array}{l}0 \\
\llcorner \\
0 \\
10 \\
0\end{array}$ & $\begin{array}{l}\stackrel{m}{N} \\
\stackrel{8}{8}\end{array}$ & \begin{tabular}{l}
0 \\
0 \\
\multirow{N}{N}{} \\
\multirow{N}{*}{}
\end{tabular} & \begin{tabular}{l}
+ \\
\multirow{0}{*}{} \\
$\infty$ \\
$\infty$
\end{tabular} & 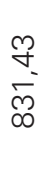 & $\begin{array}{l}9 \\
0 \\
0 \\
0 \\
\infty\end{array}$ & 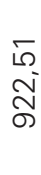 & $\begin{array}{l}\llcorner 0 \\
\infty \\
\infty \\
\varnothing \\
\mathscr{0}\end{array}$ \\
\hline & & $\begin{array}{l}N \\
0 \\
\sigma\end{array}$ & $\begin{array}{l}\bar{\xi} \\
\underline{\underline{\Sigma}}\end{array}$ & $\begin{array}{l}\text { ア } \\
6 \\
6 \\
0 \\
0\end{array}$ & 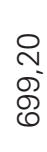 & \begin{tabular}{l}
$\overline{6}$ \\
$\stackrel{N}{N}$ \\
\multirow{N}{*}{}
\end{tabular} & $\begin{array}{l}\infty \\
0 \\
0 \\
\infty \\
\infty\end{array}$ & $\stackrel{+}{m}$ & 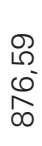 & $\begin{array}{l}\text { ㅁ } \\
\text { ㄱ } \\
\text { ন }\end{array}$ & 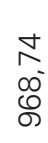 \\
\hline & & $\begin{array}{l}\overline{0} \\
\frac{0}{0} \\
\text { 을 }\end{array}$ & $\begin{array}{l}\bar{\xi} \\
\sum_{\underline{\underline{E}}}\end{array}$ & $\begin{array}{l}\bar{N} \\
0 \\
\mathcal{D}^{\prime} \\
0\end{array}$ & $\begin{array}{l}\text { ద } \\
\infty \\
\infty \\
\varnothing\end{array}$ & 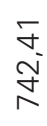 & $\begin{array}{l}\bar{L} \\
0 \\
0 \\
\Gamma\end{array}$ & \begin{tabular}{l}
\multirow{N}{N}{} \\
$\tilde{\infty}$ \\
$\infty$
\end{tabular} & $\begin{array}{l}0 \\
\stackrel{0}{0} \\
0 \\
\infty\end{array}$ & \begin{tabular}{l} 
N \\
\multirow{N}{N}{} \\
N
\end{tabular} & $\begin{array}{l}\text { P } \\
\infty \\
0 \\
\mathscr{Q} \\
8\end{array}$ \\
\hline \multicolumn{2}{|c|}{ 定 } & & $\begin{array}{l}\overline{\underline{\xi}} \\
\underline{\underline{z}}\end{array}$ & 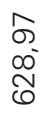 & $\begin{array}{l}\text { ঠ } \\
\text { వ' } \\
0 \\
\varnothing\end{array}$ & $\begin{array}{l}0 \\
E \\
\overline{1}\end{array}$ & $\begin{array}{l}\text { D } \\
\infty \\
\text { న్ } \\
\end{array}$ & $\frac{\infty}{\mathscr{\rho}^{-}}$ & 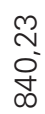 & $\begin{array}{l}\text { N } \\
\underset{\infty}{+} \\
\infty \\
\infty\end{array}$ & 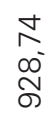 \\
\hline 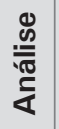 & 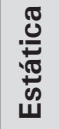 & & $\begin{array}{l}\overline{\underline{E}} \\
\underline{\underline{\underline{z}}}\end{array}$ & $\begin{array}{l}m \\
\stackrel{m}{\sigma} \\
\stackrel{\sigma}{\sigma}\end{array}$ & $\begin{array}{l}\text { ఠ } \\
\text { 응 }\end{array}$ & $\begin{array}{l}\circ \\
0 \\
0 \\
\text { లె }\end{array}$ & $\begin{array}{l}\infty \\
0 \\
0 \\
0 \\
0 \\
\llcorner\end{array}$ & 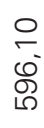 & 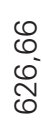 & مَ & $\begin{array}{l}\infty \\
\text { m } \\
\infty \\
\infty \\
0\end{array}$ \\
\hline & $\mathbf{N}$ & & $\underline{\xi}$ & $\approx$ & $\ddot{\theta}$ & $\stackrel{\llcorner}{\sim}$ & $\stackrel{\Xi}{ }$ & $m$ & $\stackrel{\sim}{\sim}$ & $\mp$ & 으 \\
\hline
\end{tabular}




\begin{tabular}{|c|c|c|c|c|c|c|c|c|c|c|c|}
\hline \multicolumn{2}{|c|}{ 主 } & & $\sum_{\underline{\underline{E}}}^{\bar{\xi}}$ & 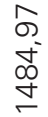 & 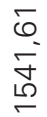 & $\begin{array}{l}\text { 워 } \\
\infty 0 \\
\text { م } \\
\stackrel{0}{\leftarrow}\end{array}$ & $\begin{array}{l}\bar{n} \\
\omega \\
0 \\
0 \\
0\end{array}$ & $\begin{array}{l}m \\
m \\
\stackrel{2}{N} \\
\end{array}$ & 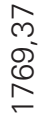 & 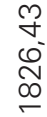 & $\begin{array}{l}0 \\
\stackrel{1}{0} \\
\text { ஸ் } \\
\infty \\
\infty\end{array}$ \\
\hline $\bar{u}$ & ? & & $\sum_{\underline{\underline{E}}}^{\bar{E}}$ & $\begin{array}{l}\text { ̊̊ } \\
\text { ஸ̊ } \\
\text { ᄋ }\end{array}$ & $\begin{array}{l}0 \\
\hat{n} \\
\stackrel{-}{\sigma}\end{array}$ & $\begin{array}{l}\frac{0}{2} \\
\frac{1}{E}\end{array}$ & $\begin{array}{l}\infty \\
\infty \\
\\
\stackrel{-}{\sim}\end{array}$ & 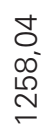 & $\begin{array}{l}\text { ๑) } \\
\stackrel{-}{\infty} \\
\stackrel{2}{\sim}\end{array}$ & $\begin{array}{l}\stackrel{\omega}{m} \\
\infty \\
m \\
m\end{array}$ & $\begin{array}{l}\bar{\kappa} \\
\infty \\
\infty \\
\infty\end{array}$ \\
\hline \multirow{5}{*}{ 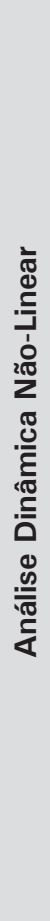 } & \multirow{5}{*}{ 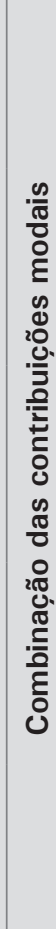 } & $\begin{array}{l}\text { م) } \\
\sigma \\
\sigma\end{array}$ & $\sum_{\underline{\underline{E}}}^{\bar{\xi}}$ & $\begin{array}{l}\hat{\sigma} \\
\sigma \\
\sigma \\
\sigma\end{array}$ & 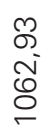 & $\begin{array}{l}\overline{0} \\
0 \\
\approx \\
\leftarrow\end{array}$ & 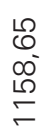 & $\begin{array}{l}\text { ঠ } \\
\text { స̃ } \\
\text { N }\end{array}$ & $\begin{array}{l}N \\
\stackrel{N}{\circ} \\
\stackrel{\sim}{N} \\
\sim\end{array}$ & 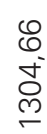 & 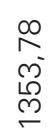 \\
\hline & & $\begin{array}{l}\dot{\sigma} \\
\sigma \\
\sigma\end{array}$ & $\sum_{\underline{\underline{E}}}^{\bar{\varepsilon}}$ & $\begin{array}{l}\hat{\sigma} \\
\leftarrow \\
0 \\
\sigma\end{array}$ & 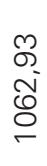 & $\begin{array}{l}\overline{0} \\
0 \\
\check{\sigma} \\
\sigma\end{array}$ & 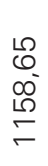 & $\begin{array}{l}\text { O } \\
\text { ㅇ. } \\
\stackrel{\text { N }}{-}\end{array}$ & $\begin{array}{l}- \\
\stackrel{1}{0} \\
\stackrel{1}{N} \\
\sim\end{array}$ & 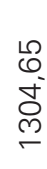 & $\begin{array}{l}\infty \\
\stackrel{\infty}{N} \\
\stackrel{m}{\infty} \\
\stackrel{m}{n}\end{array}$ \\
\hline & & $\begin{array}{l}m \\
\sigma \\
\sigma\end{array}$ & $\sum_{\underline{\underline{E}}}^{\bar{\xi}}$ & $\begin{array}{l}0 \\
0 \\
10 \\
0 \\
0\end{array}$ & 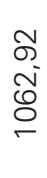 & 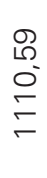 & $\begin{array}{l}\text { J } \\
\infty 0^{-} \\
\llcorner \\
\sqsubset\end{array}$ & 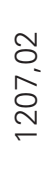 & $\begin{array}{l}\tau \\
\stackrel{1}{\circ} \\
\stackrel{0}{N} \\
\sim\end{array}$ & 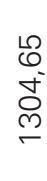 & $\begin{array}{l}\hat{N} \\
\hat{N} \\
\stackrel{ }{n}\end{array}$ \\
\hline & & $\begin{array}{l}N \\
0 \\
\sigma\end{array}$ & $\begin{array}{l}\bar{\varepsilon} \\
\underline{\underline{E}}\end{array}$ & $\begin{array}{l}0 \\
\llcorner \\
\llcorner \\
\circ \\
\circ\end{array}$ & 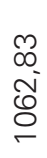 & $\begin{array}{l}\sqrt{0} \\
0 \\
\simeq \\
\approx\end{array}$ & $\begin{array}{l}\infty \\
\llcorner \\
\infty \\
\infty \\
\llcorner \\
\check{\leftarrow}\end{array}$ & $\begin{array}{l}\text { D } \\
0 \\
0 \\
\stackrel{0}{ } \\
-\end{array}$ & $\begin{array}{l}\infty \\
0 \\
\Gamma^{-} \\
\stackrel{\sim}{N} \\
\sim\end{array}$ & 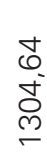 & $\begin{array}{l}\hat{N} \\
\stackrel{N}{ } \\
\stackrel{\leftrightarrow}{-}\end{array}$ \\
\hline & & $\begin{array}{l}- \\
\frac{0}{0} \\
\text { 을 }\end{array}$ & $\sum_{\underline{\underline{E}}}^{\bar{\varepsilon}}$ & 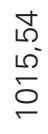 & 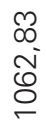 & 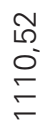 & $\begin{array}{l}\infty \\
\llcorner \\
\infty \\
\infty \\
\llcorner \\
\mp\end{array}$ & 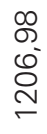 & $\begin{array}{l}0 \\
\bullet \\
\llcorner \\
\stackrel{\rho}{\Omega} \\
\sim\end{array}$ & 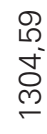 & $\begin{array}{l}\stackrel{\circ}{N} \\
\stackrel{N}{\Omega} \\
\stackrel{m}{-}\end{array}$ \\
\hline \multicolumn{2}{|c|}{ 完 } & & $\begin{array}{l}\bar{\xi} \\
\underline{\underline{E}}\end{array}$ & $\frac{m}{\stackrel{m}{n}}$ & $\begin{array}{l}0 \\
\sigma \\
\sigma \\
\sigma\end{array}$ & $\begin{array}{l}8 \\
\circ 0 \\
0 \\
0 \\
\circ\end{array}$ & $\begin{array}{l}\check{テ} \\
\check{\mp} \\
\mp\end{array}$ & 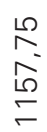 & 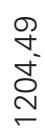 & $\begin{array}{l}\bar{\sigma} \\
\bar{\delta} \\
\stackrel{5}{\sigma}\end{array}$ & 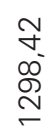 \\
\hline 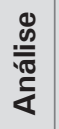 & 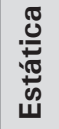 & & $\underline{\underline{\underline{E}}}$ & $\frac{N}{N}$ & $\begin{array}{l}\stackrel{\infty}{\sim} \\
\stackrel{5}{\circ}\end{array}$ & 志 & $\begin{array}{l}8 \\
\stackrel{8}{\nwarrow} \\
\infty\end{array}$ & $\begin{array}{l} \pm \\
\stackrel{5}{\circ} \\
\infty\end{array}$ & $\begin{array}{l}\stackrel{\llcorner}{N} \\
\infty \\
\infty \\
\infty\end{array}$ & $\begin{array}{l}\text { হ } \\
\text { ন }\end{array}$ & 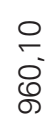 \\
\hline & $\mathbf{N}$ & & ह & の & $\infty$ & $r$ & 0 & ما & $\nabla$ & $m$ & $N$ \\
\hline
\end{tabular}




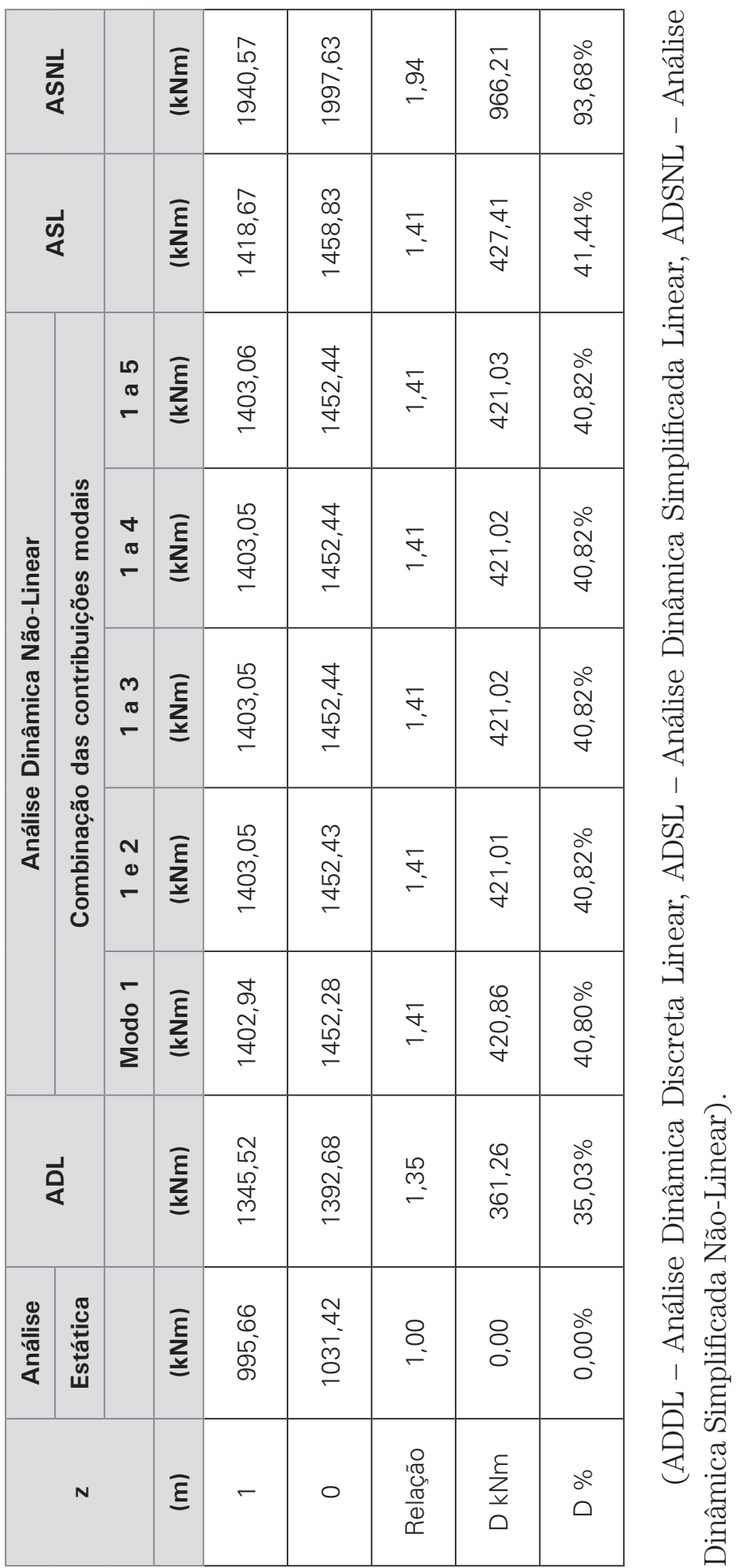




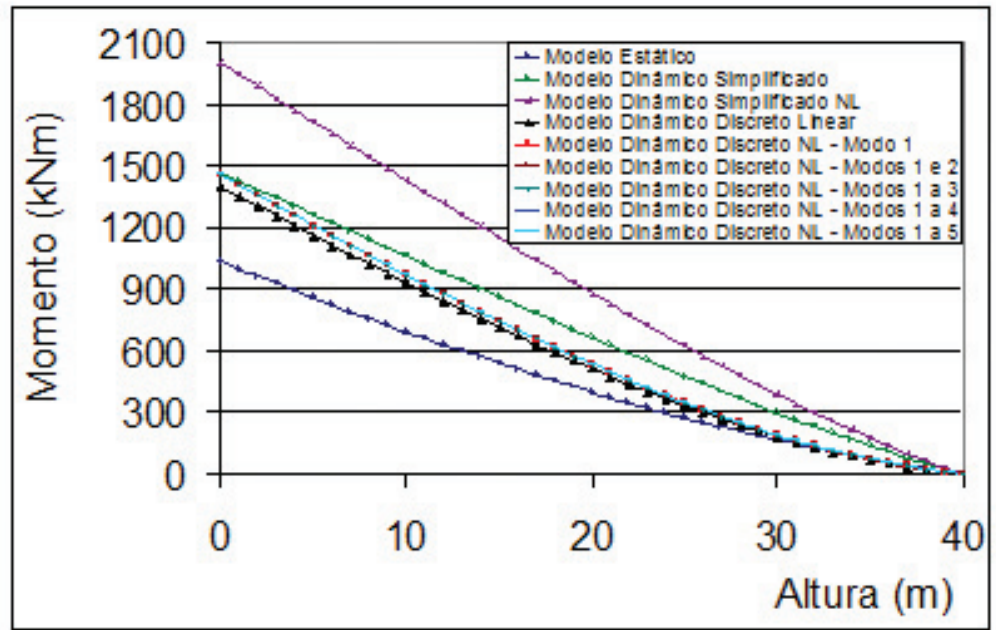

Figura $7 \cdot 27$ - Ação do vento na estrutura $4(\zeta=0,01)$.

A forma modal obtida pelo expoente da Eq. (6.6), aproxima-se bem da forma modal do modelo não-linear pelo MEF e da função proposta neste trabalho, como apresentado na Tabela 7.29.

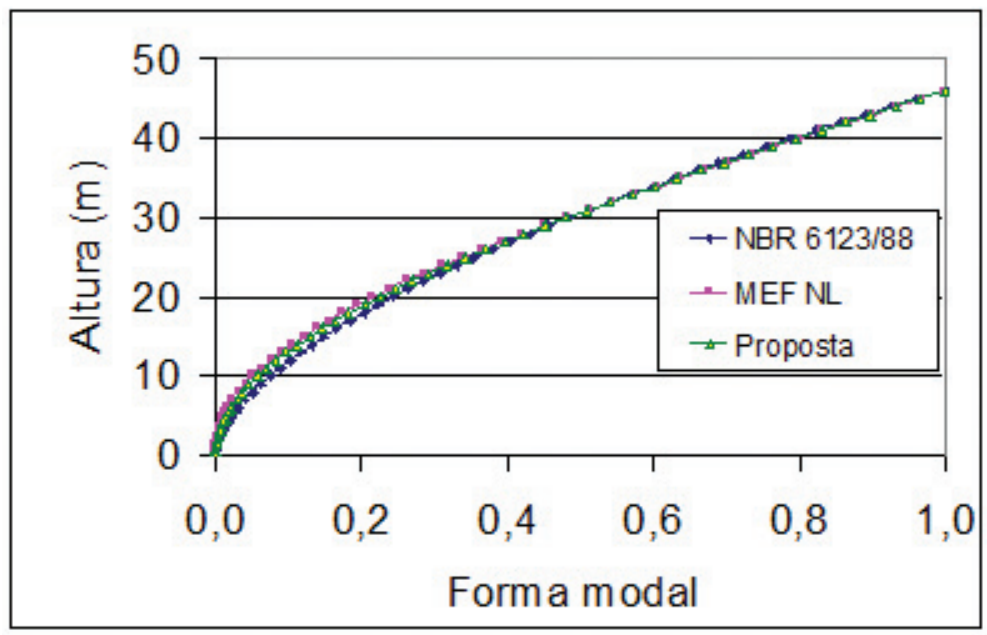

Expoente da forma modal da NBR 6123/88: $\gamma=1,7$

Figura 7.28 - Formas modais. 


\subsection{ESTRUTURA 5 - POSTE METÁLICO DE 30 M}

Para a estrutura que se apresenta nesta seção, cabe destacar a investigação experimental de campo que foi realizada para a obtenção da frequência de fundamental da estrutura, realizada por meio da aquisição de uma série temporal de dados experimentais, cujo intuito foi aferir a solução proposta nesta Tese. O resultado obtido está descrito no item 7.5.2

\subsubsection{Dados e geometria}

Esta estrutura é um poste metálico troncônico com diâmetro superior igual a $52 \mathrm{~cm}$ e diâmetro inferior de $82 \mathrm{~cm}$. Destina-se ao suporte do sistema irradiante do sinal de telefonia móvel celular. Possui 30 metros de altura e seção circular vazada de diâmetro externo (申ext) e espessura (e) variáveis com a altura. Está instalada na cidade de Aracajú, Sergipe.

Os dados da estrutura foram coletados no campo, tendo os diâmetros sido medidos com trena metálica e a espessura do copo com aparelho de ultra-som. Para um mesmo tramo vertical foram feitas diversas medidas da espessura, obtendo-se uma média relativa ao trecho. A união dos segmentos do corpo do poste é formada pelo encaixe sucessivo, por sobreposição e aparafusamento, das partes metálicas. Cada trecho sobreposto possui $20 \mathrm{~cm}$ de extensão. Na região dessas emendas, a espessura da seção transversal corresponde à soma das medidas feitas fora da zona de sobreposição. Na Tabela 7.23 e na Figura 7.29 podem ser encontradas as propriedades e a discretização utilizadas para modelar a estrutura. A esbeltez da estrutura é de 256.

A estrutura suporta duas plataformas de trabalho; uma situada a 20 m de altura e a outra na extremidade superior. Há ainda um conjunto de antenas localizadas a $27 \mathrm{~m}$ da base e fixadas ao corpo do poste por meio de suportes metálicos. As plataformas e os suportes obedecem à composição presente na Tabela 7.24 , onde $\phi$ designa 0 diâmetro da plataforma. 
Tabela 7.23 - Estrutura 5: dados da estrutura e discretização do modelo.

\begin{tabular}{|c|c|c|c|c|c|c|c|c|}
\hline cota & fext & e & cota & fext & e & cota & fext & e \\
\hline (m) & $(\mathrm{cm})$ & $(\mathrm{cm})$ & (m) & $(\mathrm{cm})$ & $(\mathrm{cm})$ & (m) & $(\mathrm{cm})$ & $(\mathrm{cm})$ \\
\hline 30,00 & 52,00 & 0,60 & 20,00 & 62,00 & 0,60 & 10,00 & 72,00 & 0,76 \\
\hline 29,00 & 53,00 & 0,60 & 19,00 & 63,00 & 0,60 & 9,00 & 73,00 & 0,76 \\
\hline 28,00 & 54,00 & 0,60 & 18,10 & 63,90 & 0,60 & 8,00 & 74,00 & 0,76 \\
\hline 27,00 & 55,00 & 0,60 & 17,90 & 64,10 & 0,60 & 7,00 & 75,00 & 0,76 \\
\hline 26,00 & 56,00 & 0,60 & 17,00 & 65,00 & 0,60 & 6,10 & 75,90 & 0,76 \\
\hline 25,00 & 57,00 & 0,60 & 16,00 & 66,00 & 0,60 & 5,90 & 76,10 & 0,76 \\
\hline 24,10 & 57,90 & 0,60 & 15,00 & 67,00 & 0,60 & 5,00 & 77,00 & 0,76 \\
\hline 23,90 & 58,10 & 0,60 & 14,00 & 68,00 & 0,60 & 4,00 & 78,00 & 0,76 \\
\hline 23,00 & 59,00 & 0,60 & 13,00 & 69,00 & 0,60 & 3,00 & 79,00 & 0,76 \\
\hline 22,00 & 60,00 & 0,60 & 12,10 & 69,90 & 0,60 & 2,00 & 80,00 & 0,76 \\
\hline 21,00 & 61,00 & 0,60 & 11,90 & 70,10 & 0,76 & 1,00 & 81,00 & 0,76 \\
\hline & & & & & & 0,00 & 82,00 & 0,76 \\
\hline
\end{tabular}


Tabela 7.24 - Estrutura 5: composição da plataforma e suporte.

\begin{tabular}{|c|c|}
\hline Plataforma $\mathrm{f}=2,5 \mathrm{~m}$ & Massa $(\mathrm{kg})$ \\
\hline Chapa piso & 116 \\
\hline Chapa lateral piso & 46 \\
\hline Perfil U 150x12,2 - Guarda-Corpo & 96 \\
\hline Cantoneira L 102×76×6,4 - Guarda-Corpo & 68 \\
\hline Cantoneira L 102×76×6,4 - Transversais - Guarda-Corpo & 77 \\
\hline Cantoneira L102x76×6,4 - Suporte do piso & 43 \\
\hline Anel inferior da plataforma & 14 \\
\hline Emendas & 3 \\
\hline Parafusos do Guarda-Corpo & 5 \\
\hline Cantoneira L 152×102×9,5 - Suporte inferior da plataforma & 33 \\
\hline Total $=$ & 500 \\
\hline Conjunto suporte para antena & Massa $(\mathbf{k g})$ \\
\hline Tubo $f=1^{\prime}(25,4 \mathrm{~mm})$ & 6 \\
\hline Cantoneira (L203×152×19) & 50 \\
\hline Grampos $\cup\left(f=1^{\prime}\right)$ & 1 \\
\hline Chapa de topo & 1 \\
\hline Total $=$ & 58 \\
\hline
\end{tabular}


O levantamento feito no local revelou a presença de antenas de micro-ondas (MW) e de rádio-frequência $(\mathrm{RF})$, que estão relacionadas juntamente com os demais acessórios da estrutura na Tabela 7.25. As informações relativas às antenas foram retiradas do catálogo do fabricante.

Todos os dispositivos mencionados anteriormente representam massas e forças concentradas adicionais à da estrutura, compostas conforme disposto na Tabela 7.25. A Tabela 7.26 apresenta os parâmetros estruturais e dos dispositivos existentes para o cálculo da ação do vento, o peso específico do material da estrutura, o carregamento axial distribuído e o localizado.

Tabela 7.25 - Estrutura 5: composição das massas nodais localizadas.

\begin{tabular}{|c|c|c|c|c|c|c|c|}
\hline \multirow{2}{*}{ Dispositivo } & \multirow{2}{*}{$\begin{array}{c}\text { Massa } \\
\text { (kg/ } \\
\text { unid) }\end{array}$} & \multicolumn{2}{|c|}{$\begin{array}{l}1^{\mathrm{a}} \text { Plat } \\
\text { (20m) }\end{array}$} & \multicolumn{2}{|c|}{$\begin{array}{c}\text { Suporte } \\
(27 \mathrm{~m})\end{array}$} & \multicolumn{2}{|c|}{$\begin{array}{l}2{ }^{\text {a }} \text { Plat } \\
(30 \mathrm{~m})\end{array}$} \\
\hline & & Qtde & (kg) & Qtde & $(\mathrm{kg})$ & Qtde & (kg) \\
\hline Antena RF 2,6 m & 19 & 2 & 37 & 3 & 56 & 1 & 19 \\
\hline Antena RF 1,23 m & 4 & 1 & 4 & 0 & 0 & 1 & 4 \\
\hline Antena MW & 19 & 2 & 38 & 0 & 0 & 0 & 0 \\
\hline Plataforma & 500 & 1 & 500 & 0 & 0 & 1 & 500 \\
\hline $\begin{array}{l}\text { Suporte para } \\
\text { antenas }\end{array}$ & 58 & 6 & 345 & 3 & 173 & 6 & 345 \\
\hline $\begin{array}{l}\text { tubos } \phi=1^{\prime} \\
(25,4 \mathrm{~mm}) \\
\text { (Balizador) }\end{array}$ & 6 & 0 & 0 & 0 & 0 & 1 & 6 \\
\hline $\begin{array}{c}\text { tubos } \phi=3 / 4^{\prime} \\
(19 \mathrm{~mm})(\mathrm{PR})\end{array}$ & 6 & 0 & 0 & 0 & 0 & 1 & 6 \\
\hline Total $(\mathrm{kg})=$ & & & 924 & & 228 & & 880 \\
\hline
\end{tabular}

(PR = Pára-raios, MW = Micro-ondas, $\mathrm{RF}$ = rádio-frequência, Plat. $=$ Plataforma) 
Tabela 7.26 - Estrutura 5: carregamento axial localizado e características dos dispositivos.

\begin{tabular}{|c|c|c|c|c|}
\hline Dispositivo & $\begin{array}{l}\text { Área } \\
\text { frontal }\end{array}$ & $\mathrm{Ca}$ & Cota & $\begin{array}{c}\text { Peso, peso } \\
\text { distribuído ou } \\
\text { peso }\end{array}$ \\
\hline Poste & Variável & 0,6 & de 0 a $30 \mathrm{~m}$ & $77 \mathrm{kN} / \mathrm{m}^{3}$ \\
\hline Escada & $0,05 \mathrm{~m}^{2} / \mathrm{m}$ & 2,0 & de 0 a $30 \mathrm{~m}$ & $0,15 \mathrm{kN} / \mathrm{m}$ \\
\hline Cabos & $0,15 \mathrm{~m}^{2} / \mathrm{m}$ & 1,2 & de 0 a $30 \mathrm{~m}$ & $0,25 \mathrm{kN} / \mathrm{m}$ \\
\hline 1a Plataforma & $2,60 \mathrm{~m}^{2}$ & 2,0 & & \\
\hline $\begin{array}{c}\text { Antenas da } 1^{a} \\
\text { plataforma }\end{array}$ & $1,99 \mathrm{~m}^{2}$ & 1,0 & $20 \mathrm{~m}$ & $9,06 \mathrm{kN}$ \\
\hline $\begin{array}{c}\text { Antenas } \\
\text { intermediárias }\end{array}$ & $2,11 \mathrm{~m}^{2}$ & 1,0 & \multirow{2}{*}{$27 \mathrm{~m}$} & \multirow{2}{*}{$2,24 \mathrm{kN}$} \\
\hline $\begin{array}{c}\text { Suportes } \\
\text { intermediários }\end{array}$ & $0,56 \mathrm{~m}^{2}$ & 2,0 & & \\
\hline 2 a Plataforma & $2,36 \mathrm{~m}^{2}$ & 2,0 & \multirow[b]{2}{*}{$30 \mathrm{~m}$} & \multirow[b]{2}{*}{$8,63 \mathrm{kN}$} \\
\hline $\begin{array}{c}\text { Antenas da } 2 \text { a } \\
\text { plataforma }\end{array}$ & $0,90 \mathrm{~m}^{2}$ & 1,0 & & \\
\hline
\end{tabular}

$(\mathrm{Ca}=$ Coeficiente de arrasto $)$

A geometria da estrutura e os dispositivos existentes foram esquematicamente representados na Figura 7.29. Da Figura 7.30 à Figura 7.36 são apresentadas imagens fotográficas do corpo, do carregamento instalado e do entorno da estrutura. A Figura 7.29 mostra detalhe da base da estrutura. 


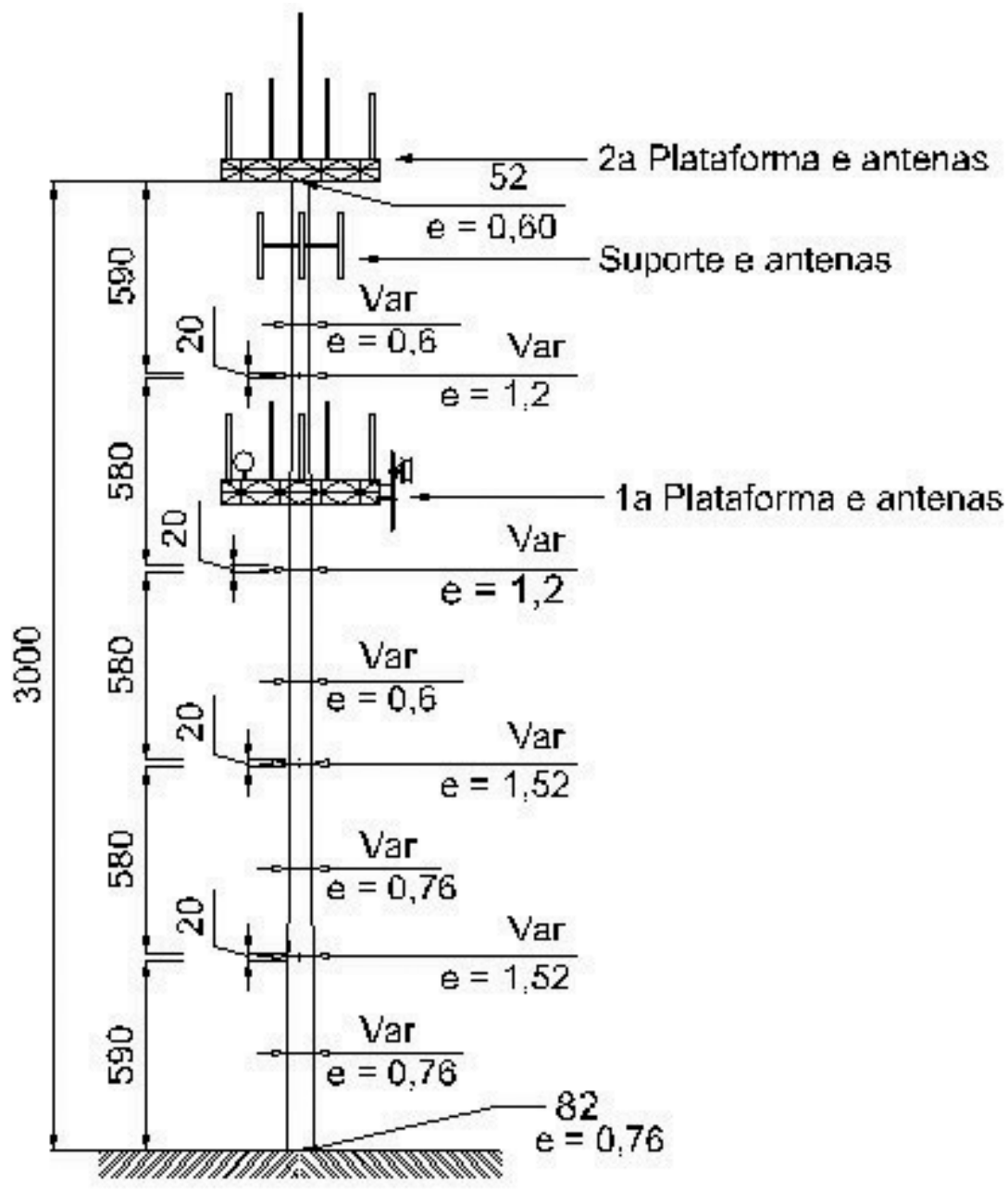

Figura 7.29 - Estrutura 5: geometria - Medidas em centímetro. 

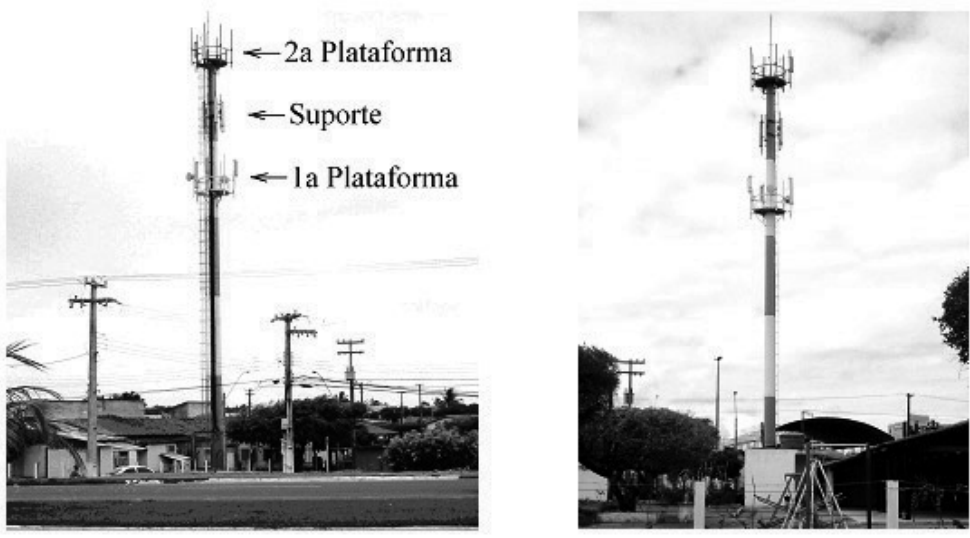

Figura 7.30 - Estrutura 5. Vista fotográfica geral.
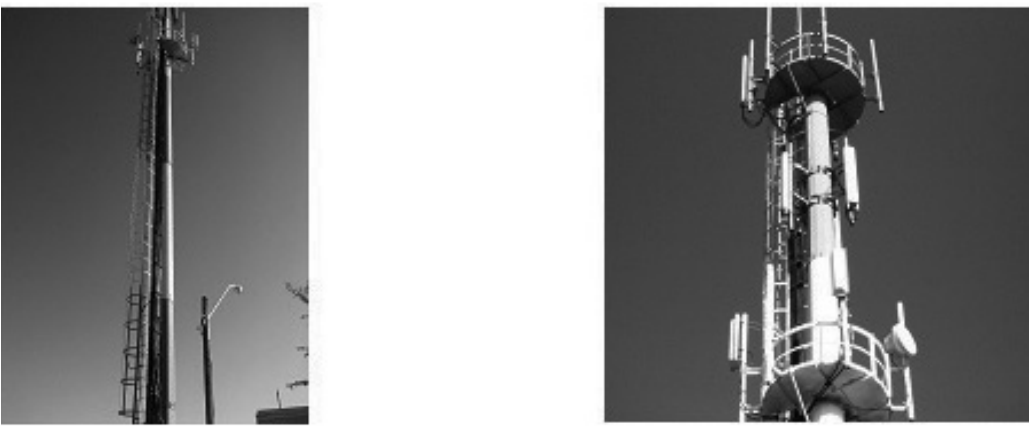

Figura 7.31 - Estrutura 5: Corpo da estrutura e carregamento.

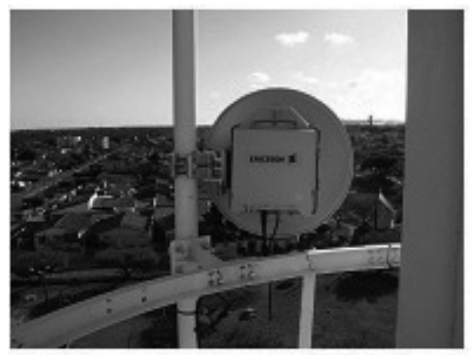

(a) Micro-ondas

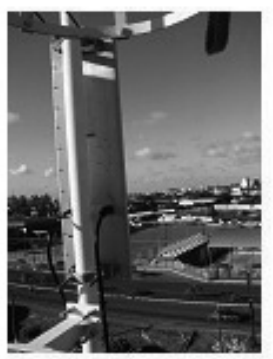

(b) Rádio Freqüência

Figura 7.32 - Estrutura 5: detalhe das antenas instaladas. 


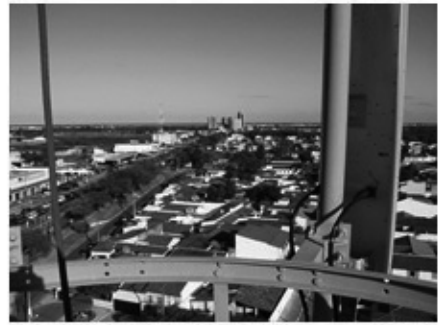

(a) Vista para o Leste

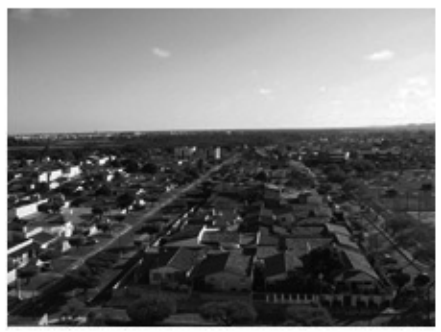

(c) Vista para o Oeste

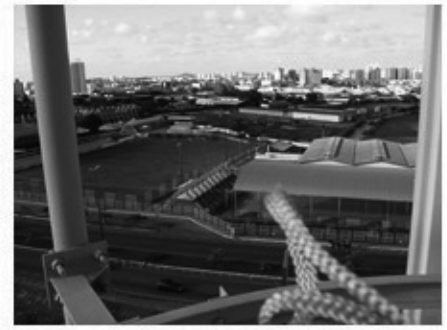

(b) Vista para o norte

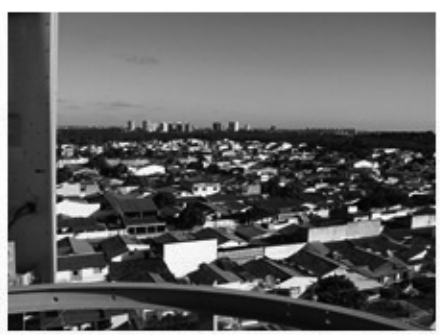

(d) Vista para o Sul

Figura 7.33 - Estrutura 5: entorno da estrutura.
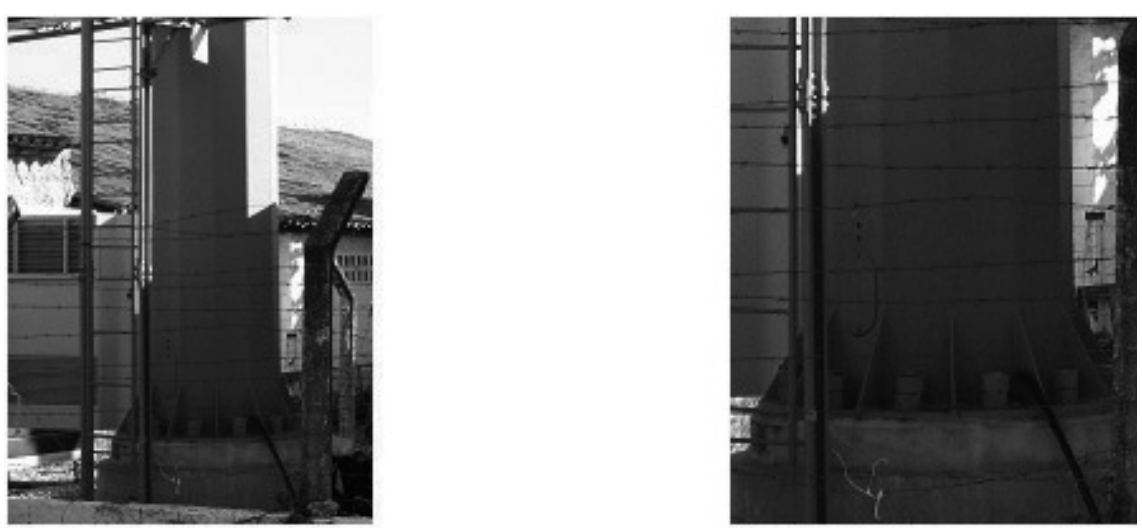

Figura 7.34 - Estrutura 5: detalhe da base. 


\subsubsection{Investigação experimental da frequência natural de vibração da estrutura}

A investigação da frequência natural da estrutura, sob excitação ambiente, foi realizada utilizando acelerômetro do tipo piezoresistivo, fabricado pela Bruel \& Kjaer, com resposta DC, com sensibilidade de $1021 \mathrm{mV} / \mathrm{g}$, com cabo integrado, capaz de medir acelerações entre \pm 2 g. Esse dispositivo foi fixado à superfície da extremidade superior do poste, conforme se vê na Figura 7.35.
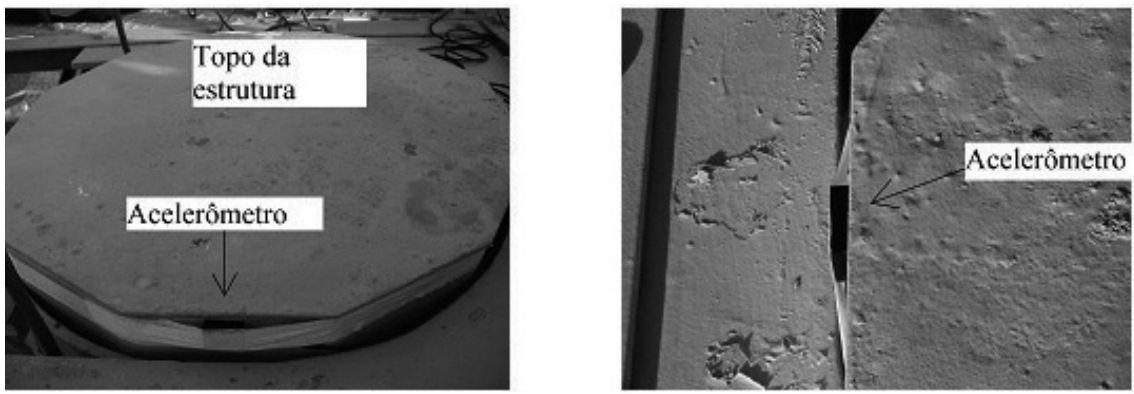

Figura 7.35 - Estrutura 5: instrumentação.

A aquisição dos dados foi feita pelo sistema ADS2000, AdDados, da Lynx informática, que estava conectado a um microcomputador portátil para gravação dos sinais. Os equipamentos foram conduzidos ao alto do poste, onde foram depositados sobre a superfície da plataforma de trabalho, conforme pode ser visto na Figura 7.36, e protegidos das intempéries. O sistema de energia elétrica da estação serviu como fonte para alimentar os equipamentos eletrônicos. 


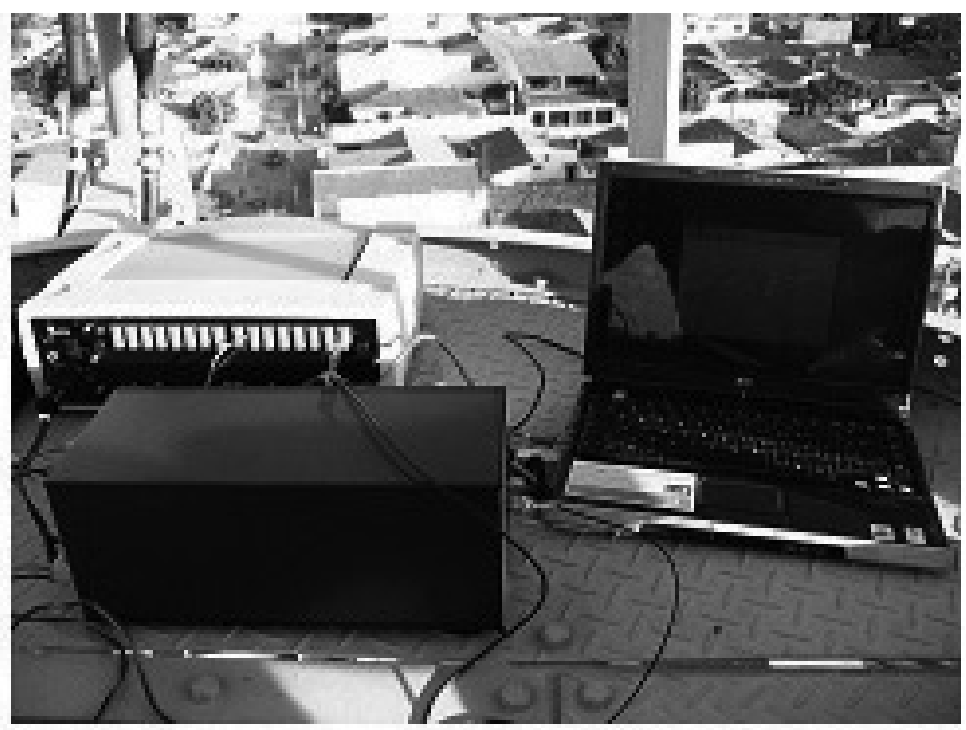

Figura 7.36 - Estrutura 5: sistema instalado na estrutura para aquisição dos dados.

A aquisição dos sinais foi realizada com taxa de $50 \mathrm{~Hz}$ e teve duração de 40 h 33 min 22 s, iniciando-se no dia 11 de dezembro de 2007, às 18 h 30 min 23 s. A série temporal da aceleração pode ser vista na Figura 7.37.

Percebeu-se que a estrutura estava sob suficiente excitação do vento, tendo inclusive ocorrido chuva e ventos fortes durante o período em que foi instrumentada.

A frequência fundamental da estrutura foi obtida a partir da série temporal da aquisição dos sinais pela Transformada de Fourier (FFT) no programa AqDAnalysis 7.02. O resultado obtido foi de 0,53 Hz. Na Figura 7.38 encontra-se a análise do sinal no domínio da frequência. 


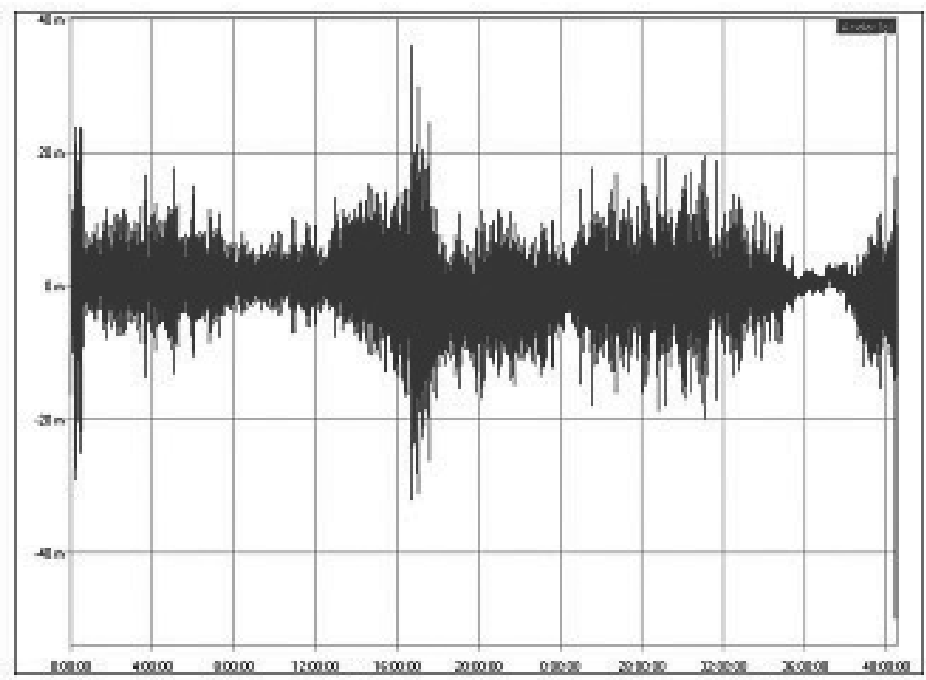

Figura 7.37 - Estrutura 5: série temporal de aceleração.

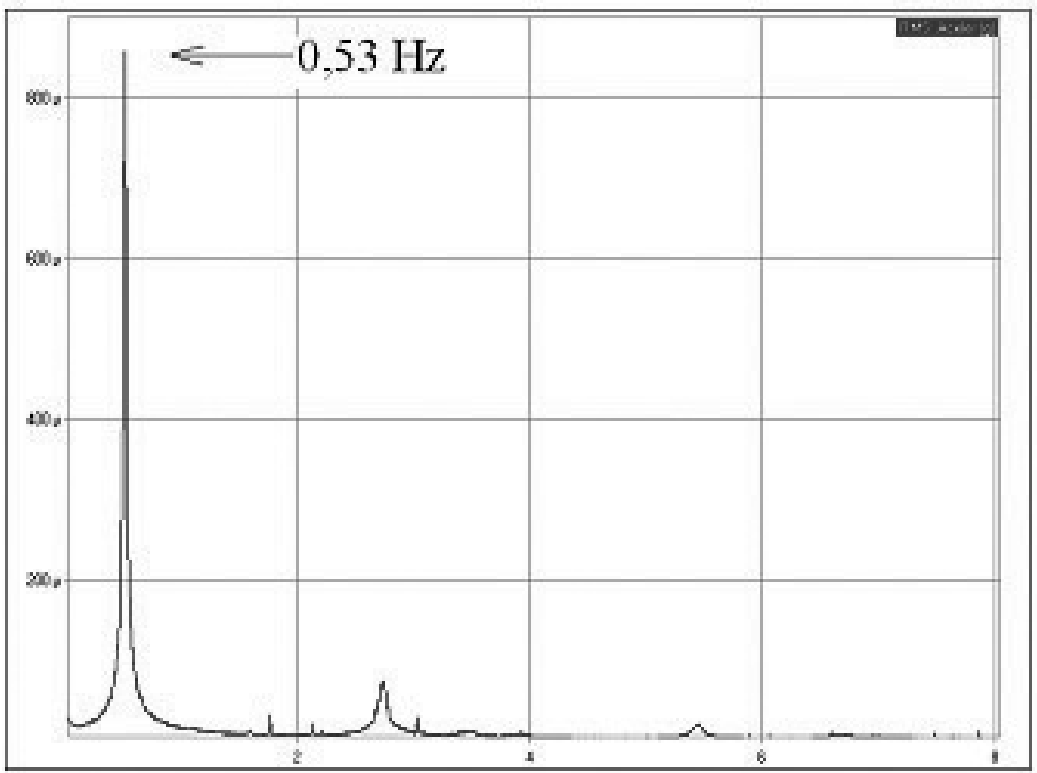

Figura 7.38 - Estrutura 5: sinal no domínio da frequência. 


\subsubsection{Modelagem por Elementos Finitos}

A modelagem por Elementos Finitos acompanhou os critérios já utilizados nas estruturas precedentes. No entanto, há um importante detalhe que aparece na discretização deste modelo. As regiões de ligações foram tratadas como elementos de barra de seção variável de 0,2 m de comprimento e espessura correspondente à soma das espessuras das seções que estavam imediatamente acima e abaixo da zona de emenda.

Na Figura 7.39 se encontra o modelo em Elementos Finitos. com uma vista tridimensional, uma lateral e a discretização da estrutura que conta com 40 elementos de barra. Os modos de vibração e as frequências obtidas pelo Método dos Elementos Finitos são as constantes na Figura 7.40.

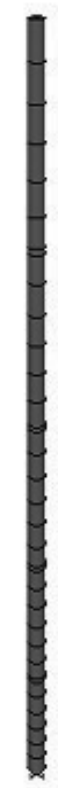

(a) 3D

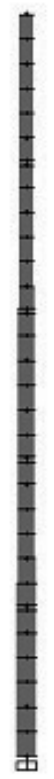

(b) Vista lateral

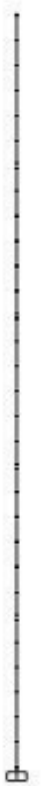

(c) Discretização

(NLG = Não-Linearidade Geométrica)

Figura 7.39 - Estrutura 5: modelo por Elementos Finitos. 


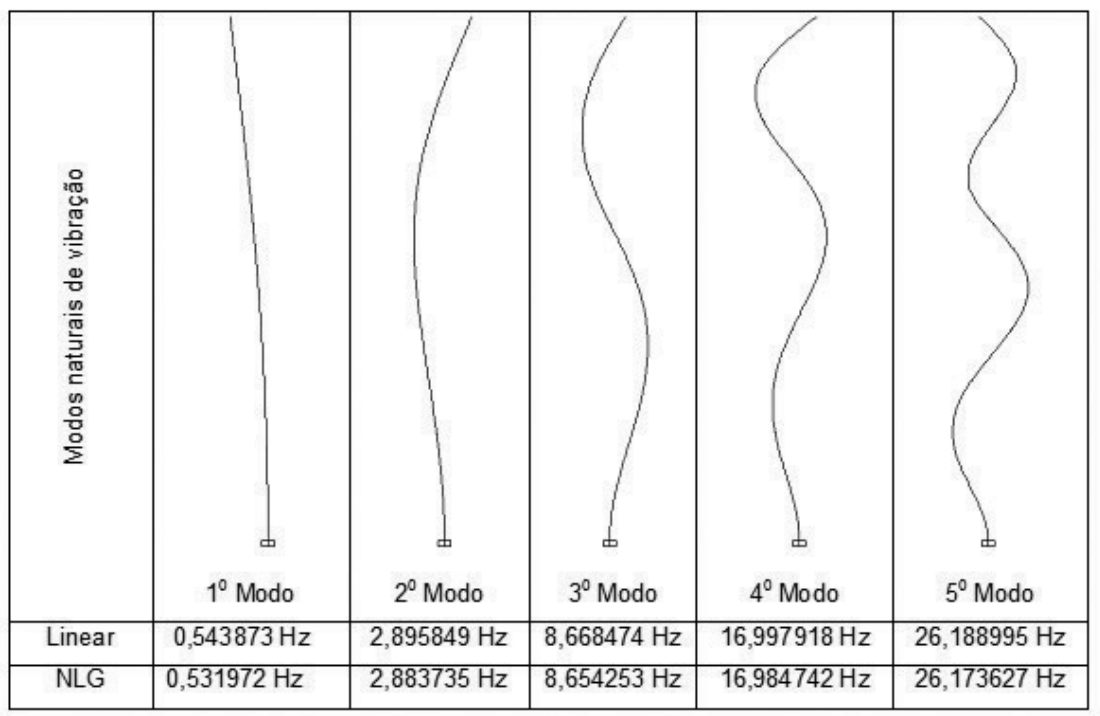

Figura 7.40 - Estrutura 5: modos naturais de vibração.

\subsubsection{Aplicação do método proposto}

\subsubsection{Definição dos parâmetros}

Os dados para aplicação do método são:

- módulo de elasticidade: $\mathrm{E}=205 \mathrm{GPa}$,

- densidade do aço: $\rho=7850 \mathrm{~kg} / \mathrm{m} 3$,

- $\quad$ massa concentrada no topo: $\mathrm{m}_{0}=880 \mathrm{~kg}$;

- $\quad$ massa concentra a $27 \mathrm{~m}$ da altura: $\mathrm{m}_{10}=228 \mathrm{~kg}$;

- $\quad$ massa concentrada a 20 m de altura: $\mathrm{m}_{11}=924 \mathrm{~kg}$;

- massa distribuída: $\mathrm{m}_{\mathrm{e}}=40 \mathrm{~kg} / \mathrm{m}$.

Em toda a estrutura o diâmetro externo varia linearmente com a altura seguindo a expressão

$$
\mathrm{D}(\mathrm{x})=\frac{\mathrm{D}_{9}-\mathrm{D}_{0}}{\mathrm{~L}} \mathrm{x}+\mathrm{D}_{0}
$$


onde $\mathrm{D}_{9}$ é o diâmetro da extremidade superior e $\mathrm{D}_{0}$ é o diâmetro da extremidade inferior.

As ordenadas e as espessuras das seções, dos trechos de interesse definidos na geometria são as seguintes.

Na base, quando $\mathrm{x}=0$, tem-se: $\mathrm{D}_{0}=82 \mathrm{~cm}, \mathrm{e}_{1}=0,76 \mathrm{~cm}$.

No primeiro segmento, da base do poste até $\mathrm{L}_{1}=5,90 \mathrm{~m}$, tem-se $\mathrm{e}_{1}=0,76 \mathrm{~cm}$.

No segundo segmento, entre $L_{1}$ e $L_{2}=6,10 \mathrm{~m}$, define-se $\mathrm{e}_{2}=1,52 \mathrm{~cm}$.

No terceiro segmento, entre $L_{2}$ e $L_{3}=11,90 \mathrm{~m}$, tem-se $\mathrm{e}_{3}=0,76 \mathrm{~cm}$.

No quarto segmento, entre $\mathrm{L}_{3}$ e $\mathrm{L}_{4}=12,10 \mathrm{~m}$, tem-se $\mathrm{e}_{4}=1,52 \mathrm{~cm}$.

No quinto segmento, entre $\mathrm{L}_{4}$ e $\mathrm{L}_{5}=17,90 \mathrm{~m}$, tem-se $\mathrm{e}_{5}=0,60 \mathrm{~cm}$.

No sexto segmento, entre $\mathrm{L}_{5}$ e $\mathrm{L}_{6}=18,10 \mathrm{~m}$, tem-se $\mathrm{e}_{6}=1,20 \mathrm{~cm}$.

No sétimo segmento, entre $\mathrm{L}_{6}$ e $\mathrm{L}_{7}=23,90 \mathrm{~m}$, tem-se $\mathrm{e}_{7}=0,60 \mathrm{~cm}$.

No oitavo segmento, entre $\mathrm{L}_{7}$ e $\mathrm{L}_{8}=24,10 \mathrm{~m}$, tem-se $\mathrm{e}_{8}=0,60 \mathrm{~cm}$.

No nono segmento, entre $\mathrm{L}_{8}$ e $\mathrm{L}=30,00 \mathrm{~m}$, tem-se $\mathrm{e}_{9}=0,60 \mathrm{~cm}$.

As propriedades geométricas, como diâmetro interno, área e momento de inércia das seções, foram obtidas com as seguintes expressões gerais:

$$
\mathrm{d}_{\mathrm{i}}(\mathrm{x})=\mathrm{D}(\mathrm{x})-2 \mathrm{e}_{\mathrm{i}}
$$




$$
\begin{aligned}
& \mathrm{A}_{\mathrm{i}}(\mathrm{x})=\frac{\pi}{4}\left(\mathrm{D}(\mathrm{x})^{2}-\mathrm{d}_{\mathrm{i}}(\mathrm{x})^{2}\right) \\
& \mathrm{I}_{\mathrm{i}}(\mathrm{x})=\frac{\pi}{64}\left(\mathrm{D}(\mathrm{x})^{4}-\mathrm{d}_{\mathrm{i}}(\mathrm{x})^{4}\right)
\end{aligned}
$$

onde i caracteriza o segmento analisado.

\subsubsection{Cálculo da massa generalizada}

A massa generalizada oriunda das massas distribuídas de cada segmento, foi calculada fazendo-se

$$
\mathrm{m}_{\mathrm{i}}=\int_{\mathrm{L}_{\mathrm{i}-1}}^{\mathrm{L}_{\mathrm{i}}} \mathrm{m}_{\mathrm{i}}(\mathrm{x}) \phi(\mathrm{x})^{2} \mathrm{dx}, \text { com } \mathrm{m}_{\mathrm{i}}(\mathrm{x})=\mathrm{A}_{\mathrm{i}}(\mathrm{x}) \rho+\mathrm{m}_{\mathrm{e}}
$$

com i, i $=1,2 \ldots 9$.

E a generalização das massas concentradas foi obtida por

$$
\begin{aligned}
& \mathrm{m}_{10}=\mathrm{m}_{\mathrm{x}} \phi\left(\mathrm{x}_{10}\right)^{2}, \text { com } \mathrm{x}_{10}=27,00 \mathrm{~m} ; \\
& \mathrm{m}_{11}=\mathrm{m}_{\mathrm{xI}} \phi\left(\mathrm{x}_{11}\right)^{2}, \text { com } \mathrm{x}_{11}=20,00 \mathrm{~m} ;
\end{aligned}
$$

A massa distribuída generalizada fica então

$$
\mathrm{m}_{\mathrm{R}}=\sum_{\mathrm{i}=1}^{11} \mathrm{~m}_{\mathrm{i}}
$$

E a massa generalizada total

$$
\mathrm{M}=\mathrm{m}_{0}+\mathrm{m}_{\mathrm{R}}
$$




\subsubsection{Cálculo da rigidez generalizada}

\section{Rigidez geométrica generalizada}

Os esforços normais concentrados são dados por:

$$
\begin{aligned}
& \mathrm{F}_{0}=\mathrm{m}_{0} \mathrm{~g}, \\
& \mathrm{~F}_{11}=\mathrm{m}_{\mathrm{XI}} \mathrm{g} \\
& \mathrm{F}_{10}=\mathrm{m}_{\mathrm{x}} \mathrm{g}
\end{aligned}
$$

$\mathrm{E}$ as forças normais devidas a massa distribuída dos segmentos, por

$$
F_{i}=\int_{L_{i-1}}^{L_{i}} m_{1}(x) g d x
$$

A força normal generalizada é, então:

$$
\mathrm{F}=\sum_{\mathrm{i}=0}^{11} \mathrm{~F}_{\mathrm{i}}
$$

Com isso as parcelas das rigidezes geométricas foram calculadas pelas seguintes expressões:

$$
\begin{aligned}
& \mathrm{K}_{\mathrm{g} 9}=\int_{\mathrm{L}_{8}}^{\mathrm{L}}\left[\mathrm{F}_{0}+\mathrm{F}_{10}+\mathrm{m}_{\mathrm{IX}}(\mathrm{x})(\mathrm{L}-\mathrm{x}) \mathrm{g}\left(\frac{\mathrm{d}}{\mathrm{dx}} \phi(\mathrm{x})\right)^{2}\right], \\
& \mathrm{K}_{\mathrm{g} 8}=\int_{\mathrm{L}_{7}}^{\mathrm{L}_{8}}\left[\mathrm{~F}_{0}+\mathrm{F}_{10}+\mathrm{F}_{9}+\mathrm{m}_{\mathrm{VIII}}(\mathrm{x})\left(\mathrm{L}_{8}-\mathrm{x}\right) \mathrm{g}\left(\frac{\mathrm{d}}{\mathrm{dx}} \phi(\mathrm{x})\right)^{2}\right],
\end{aligned}
$$

que se repete para os demais segmentos, de forma que se pode escrever 


$$
\mathrm{K}_{\mathrm{gi}}=\int_{\mathrm{L}_{\mathrm{i}-1}}^{\mathrm{L}_{\mathrm{i}}}\left[\mathrm{F}_{0}+\sum_{\mathrm{i}+1}^{10} \mathrm{~F}_{\mathrm{i}+1}+\mathrm{m}_{\mathrm{i}}(\mathrm{x})\left(\mathrm{L}_{\mathrm{i}}-\mathrm{x}\right) \mathrm{g}\left(\frac{\mathrm{d}}{\mathrm{dx}} \phi(\mathrm{x})\right)^{2}\right]
$$

Assim, a rigidez geométrica generalizada total é:

$$
\mathrm{K}_{\mathrm{g}}=\sum_{\mathrm{i}=1}^{11} \mathrm{~K}_{\mathrm{gi}}
$$

\subsection{Rigidez elástica generalizada}

Analogamente à rigidez geométrica, calcularam-se as parcelas das rigidezes convencionais generalizadas por

$$
\mathrm{K}_{0 \mathrm{i}}=\int_{\mathrm{L}_{\mathrm{i}-1}}^{\mathrm{Li}} \mathrm{EI}_{\mathrm{i}}(\mathrm{x})\left(\frac{\mathrm{d}^{2}}{\mathrm{dx}^{2}} \phi(\mathrm{x})\right)^{2} \mathrm{dx}
$$

e a rigidez elástica generalizada pelo somatório

$$
\mathrm{K}_{0}=\sum_{\mathrm{i}=1}^{11} \mathrm{~K}_{0 \mathrm{i}}
$$

\subsubsection{Cálculo da frequência}

As frequências do primeiro modo de vibração da estrutura calculadas pelo método proposto neste trabalho são: modelo linear $=$ 0,550271 Hz e modelo não-linear = 0,537826 Hz.

\subsubsection{Ação do vento}

\subsubsection{Forças estáticas devidas ao vento}

As anotações existentes na placa de identificação da estação indicam os parâmetros empregados na determinação das forças estáticas devidas a ação do vento e utilizados no dimensionamento da 
estrutura, que são: fator topográfico $S_{1}=1,0$; fator de rugosidade do terreno $\mathrm{S}_{2}$ correspondente à categoria II, classe $\mathrm{B}$; fator estatístico $\mathrm{S}_{3}$ $=1,1$; velocidade básica do vento $\mathrm{V}_{0}=30 \mathrm{~m} / \mathrm{s}$.

\section{5.6.2 Resposta dinâmica pelo modelo simplificado da NBR 6123/88}

Os parâmetros usados na determinação da resposta dinâmica pelo modelo contínuo simplificado da NBR 6123/88 foram os seguintes: largura da edificação $0,670 \mathrm{~m}$, altura da edificação $30 \mathrm{~m}$, categoria do terreno, velocidade básica do vento e fatores estatísticos como descrito no item anterior.

Para o cálculo da frequência recorreu-se à recomendação contida na Tabela 6.4 (Tabela 19 da NBR 6123/88). Com a expressão $0,29 \sqrt{\mathrm{h}}-0,4$ obtém-se uma frequência do modo fundamental igual a $0,841471 \mathrm{~Hz}$, um resultado distante $35,37 \%$ do calculado linearmente pelo Método dos Elementos Finitos e distante 36,08\% do cálculo desenvolvido pelo método proposto. Com esse resultado, a relação adimensional $\mathrm{Vp} /\left(\mathrm{f}_{1} \mathrm{~L}\right)$ torna-se igual a 0,010. Adotando a taxa de amortecimento crítico $\zeta$ igual a 0,015 chega-se a um coeficiente de amplificação dinâmica $\xi$ de 1,926. A forma de vibração do primeiro modo sugerida pela NBR6123/88 tem, para a correspondente expressão da frequência, o expoente da expressão (6.6) igual 1,7.

A segunda avaliação, usando o modelo simplificado da NBR 6123/88, feita sob não-linearidade geométrica, teve como ponto de partida a frequência de $0,531970 \mathrm{~Hz}$. Com isso, a relação adimensional $\mathrm{Vp} /\left(\mathrm{f}_{1} \mathrm{~L}\right)$ fica em 0,024, o que conduziu a um fator de amplificação dinâmica $\xi=2,180$.

\subsubsection{Resposta dinâmica pelo modelo discreto da NBR 6123/88}

A resposta dinâmica pelo modelo dinâmico discreto linear foi calculada com base na frequência e parâmetros citados no item precedente. Para esse procedimento de cálculo, a relação adimensional Vp/ $\left(\mathrm{f}_{1} \mathrm{~L}\right)$ foi de 0,023 e o coeficiente de amplificação dinâmica de 2,158. 
O cálculo da resposta dinâmica com a inclusão da não-linearidade geométrica foi feito levando-se em conta as contribuições até o $5^{\circ}$ modo de vibração. Para o primeiro modo a relação adimensional e o coeficiente de amplificação dinâmica constam no item anterior. Para os modos de 2 a 5, a relação adimensional e o coeficiente de amplificação dinâmica são, respectivamente, 0,004 e 1,634; 0,001 e 1,508; 0,001 e 1,$463 ; 0,0005$ e 1,444 .

\subsubsection{Análise dos resultados}

Os esforços normais na estrutura pelo método proposto e pelo Método dos Elementos Finitos podem ser vistos na Tabela 7.27.

Tabela 7.27 - Estrutura 5: esforço normal.

\begin{tabular}{|c|c|c|c|c|}
\hline $\mathbf{L}$ & Proposto & MEF & \multicolumn{2}{|c|}{ Diferença } \\
\hline $\mathbf{m})$ & $\mathbf{( k N )}$ & $\mathbf{( k N )}$ & Absoluta & $\mathbf{( \% )}$ \\
\hline 30,00 & 8,624000 & 8,624000 & 0,0000 & 0,000000 \\
\hline 24,10 & 17,820587 & 17,820587 & 0,0000 & $-0,000001$ \\
\hline 23,90 & 18,228409 & 18,228409 & 0,0000 & $-0,000001$ \\
\hline 18,10 & 34,636570 & 34,636570 & 0,0000 & $-0,000001$ \\
\hline 17,90 & 35,079190 & 35,079190 & 0,0000 & $-0,000001$ \\
\hline 12,10 & 42,936723 & 42,936724 & 0,0000 & $-0,000001$ \\
\hline 11,90 & 43,518196 & 43,518196 & 0,0000 & $-0,000001$ \\
\hline 6,10 & 53,486859 & 53,486860 & 0,0000 & $-0,000001$ \\
\hline 5,90 & 54,112409 & 54,112410 & 0,0000 & $-0,000001$ \\
\hline 0,00 & 64,908508 & 64,908509 & 0,0000 & $-0,000001$ \\
\hline
\end{tabular}


A frequência do primeiro modo de vibração obtida pelo método proposto, sem a consideração da não-linearidade geométrica, foi de 0,550271 Hz e pelo Método dos Elementos Finitos, de 0,543873 Hz, apresentando uma diferença de 1,18\%. Pela expressão adotada da NBR 6123/88, essa frequência corresponde a 0,841471 Hz.

A diferença entre a frequência fundamental do modelo não-linear, calculada pelo método proposto de $0,537826 \mathrm{~Hz}$, e a obtida pelo MEF de 0,531972 Hz, é de 1,10\%. Ambas as frequências coincidem, com a frequência medida experimentalmente, o que valida a aplicação da solução proposta nesta Tese.

Verificou-se bom ajustamento da forma de vibração adotada pelo método proposto e a do modelo não-linear do MEF.

A frequência calculada pela aproximação do método proposto, usando a expressão (4.21), foi de $0,408091 \mathrm{~Hz}$, apresentando uma diferença de 24,12 \% em relação ao valor exato do método e de 36,08\% em relação ao resultado obtido segundo a expressão sugerida pela NBR6123/88.

Os resultados críticos da ação do vento são encontrados na comparação entre a análise estática e a análise pelo modelo dinâmico discreto não-linear, quando a análise não-linear supera a análise estática em 1,56 vezes (55,69 \%).

A análise dinâmica discreta linear fica 54,67\% acima da análise estática. Já as resposta dinâmicas obtida com os modelos simplificados guardam entre si uma diferença de 8,47\%, com superioridade da análise não-linear.

Comparando os resultados obtidos pelas análises discretas não-lineares, verifica-se novamente uma pequena influência da contribuição dos modos de vibração que estão acima do fundamental, resultando uma diferença de 0,01\% entre a resposta dinâmica da estrutura com a consideração exclusiva do primeiro modo e a combinação que inclui também as contribuições do $2^{\circ}$ modo.

Na resposta dinâmica da estrutura com superposição das contribuições do vento médio com as flutuações devidas ao primeiro modo de vibração, as flutuações do $1^{\circ}$ modo são responsáveis por $66 \%$ da resposta dinâmica total da estrutura, conforme pode ser visto na Tabela 7.28. 
A diferença entre a frequência fundamental do modelo linear e a do modelo não-linear, de 2,19\%, elevou o coeficiente de amplificação dinâmica em 1,02\%. Com isso, o momento fletor máximo na estrutura foi acrescido de 4,99 kNm, uma diferença de 0,65\%.

A Tabela 7.29 traz o valor dos momentos máximos devidos à ação do vento das análises realizadas, comparando-as ao modelo estático.

Tabela 7.28 - Estrutura 5: momentos fletores da análise discreta não-linear.

\begin{tabular}{|c|c|c|c|c|c|c|}
\hline \multirow{2}{*}{ z } & \multirow{2}{*}{$\begin{array}{l}\text { Vento } \\
\text { Médio }\end{array}$} & \multicolumn{5}{|c|}{ Flutuações } \\
\hline & & Modo1 & Modo 2 & Modo 3 & Modo 4 & Modo 5 \\
\hline (m) & $(\mathrm{kNm})$ & (kNm) & (kNm) & (kNm) & (kNm) & (kNm) \\
\hline 30,00 & 0,00 & 0,00 & 0,00 & 0,00 & 0,00 & 0,00 \\
\hline 29,00 & 2,61 & 6,78 & 0,47 & 0,26 & 0,28 & 0,08 \\
\hline 28,00 & 5,46 & 14,35 & 0,99 & 0,53 & 0,56 & 0,16 \\
\hline 27,00 & 8,55 & 22,70 & 1,54 & 0,79 & 0,79 & 0,21 \\
\hline 26,00 & 13,27 & 33,15 & 2,13 & 0,97 & 0,81 & 0,18 \\
\hline 25,00 & 18,23 & 44,29 & 2,71 & 1,11 & 0,74 & 0,11 \\
\hline 24,10 & 22,91 & 54,88 & 3,23 & 1,20 & 0,61 & 0,03 \\
\hline 23,90 & 23,98 & 57,31 & 3,34 & 1,21 & 0,57 & 0,01 \\
\hline 23,00 & 28,98 & 68,57 & 3,82 & 1,23 & 0,35 & 0,08 \\
\hline
\end{tabular}




\begin{tabular}{|c|c|c|c|c|c|c|}
\hline \multirow{2}{*}{ Z } & \multirow{2}{*}{$\begin{array}{l}\text { Vento } \\
\text { Médio }\end{array}$} & \multicolumn{5}{|c|}{ Flutuações } \\
\hline & & Modo1 & Modo 2 & Modo 3 & Modo 4 & Modo 5 \\
\hline (m) & (kNm) & (kNm) & (kNm) & (kNm) & (kNm) & (kNm) \\
\hline 22,00 & 34,77 & 81,64 & 4,32 & 1,20 & 0,06 & 0,17 \\
\hline 21,00 & 40,80 & 95,23 & 4,77 & 1,11 & 0,26 & 0,24 \\
\hline 20,00 & 47,07 & 109,32 & 5,16 & 0,97 & 0,58 & 0,29 \\
\hline 19,00 & 56,06 & 127,10 & 5,03 & 0,56 & 0,65 & 0,16 \\
\hline 18,10 & 64,37 & 143,45 & 4,86 & 0,18 & 0,68 & 0,03 \\
\hline 17,90 & 66,25 & 147,13 & 4,81 & 0,09 & 0,68 & 0,01 \\
\hline 17,00 & 74,86 & 163,91 & 4,55 & 0,29 & 0,64 & 0,11 \\
\hline 16,00 & 84,66 & 182,88 & 4,20 & 0,70 & 0,55 & 0,22 \\
\hline 15,00 & 94,69 & 202,16 & 3,77 & 1,06 & 0,39 & 0,28 \\
\hline 14,00 & 104,95 & 221,71 & 3,26 & 1,37 & 0,20 & 0,30 \\
\hline 13,00 & 115,44 & 241,50 & 2,69 & 1,60 & 0,02 & 0,26 \\
\hline 12,10 & 125,07 & 259,50 & 2,12 & 1,74 & 0,22 & 0,19 \\
\hline 11,90 & 127,24 & 263,52 & 1,99 & 1,76 & 0,27 & 0,17 \\
\hline
\end{tabular}




\begin{tabular}{|c|c|c|c|c|c|c|}
\hline \multirow{2}{*}{ z } & \multirow{2}{*}{$\begin{array}{l}\text { Vento } \\
\text { Médio }\end{array}$} & \multicolumn{5}{|c|}{ Flutuações } \\
\hline & & Modo1 & Modo 2 & Modo 3 & Modo 4 & Modo 5 \\
\hline$(\mathrm{m})$ & (kNm) & (kNm) & (kNm) & (kNm) & (kNm) & (kNm) \\
\hline 11,00 & 137,16 & 281,75 & 1,35 & 1,79 & 0,45 & 0,06 \\
\hline 10,00 & 148,40 & 302,18 & 0,57 & 1,71 & 0,61 & 0,08 \\
\hline 9,00 & 159,84 & 322,77 & 0,26 & 1,53 & 0,72 & 0,21 \\
\hline 8,00 & 171,50 & 343,49 & 1,15 & 1,24 & 0,75 & 0,32 \\
\hline 7,00 & 183,36 & 364,31 & 2,07 & 0,85 & 0,71 & 0,38 \\
\hline 6,10 & 194,20 & 383,11 & 2,93 & 0,44 & 0,60 & 0,39 \\
\hline 5,90 & 196,64 & 387,30 & 3,12 & 0,33 & 0,57 & 0,38 \\
\hline 5,00 & 207,72 & 406,17 & 4,01 & 0,16 & 0,38 & 0,32 \\
\hline 4,00 & 220,21 & 427,18 & 5,01 & 0,76 & 0,12 & 0,20 \\
\hline 3,00 & 232,88 & 448,22 & 6,03 & 1,40 & 0,19 & 0,03 \\
\hline 2,00 & 245,70 & 469,28 & 7,06 & 2,06 & 0,53 & 0,17 \\
\hline 1,00 & 258,66 & 490,34 & 8,08 & 2,73 & 0,89 & 0,39 \\
\hline 0,00 & 271,74 & 511,40 & 9,11 & 3,41 & 1,25 & 0,61 \\
\hline
\end{tabular}




\begin{tabular}{|c|c|c|c|c|c|c|c|c|c|c|}
\hline \multicolumn{2}{|c|}{ 岕 } & & $\sum_{\underline{\underline{\Sigma}}}^{\overline{\underline{\Sigma}}}$ & $\begin{array}{l}\circ \\
\text { ○. } \\
\end{array}$ & $\begin{array}{l}\hat{\sigma} \\
\text { a. }\end{array}$ & $\begin{array}{l}m \\
\infty \\
0 \\
0\end{array}$ & 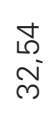 & 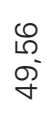 & $\begin{array}{l}\text { m. } \\
\text { No }\end{array}$ & $\begin{array}{l}\text { ది } \\
\text { ల' }\end{array}$ \\
\hline \multicolumn{2}{|c|}{ 崫 } & & $\begin{array}{l}\overline{\underline{E}} \\
\text { 兰 }\end{array}$ & $\begin{array}{l}\text { ○ } \\
\text { ○' }\end{array}$ & $\frac{\circ}{\sigma}$ & $\begin{array}{l}\text { Oे } \\
\text { ウ் }\end{array}$ & $\frac{\text { P }}{\stackrel{N}{N}}$ & 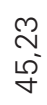 & $\frac{\text { t. }}{\dot{\sigma}}$ & $\begin{array}{l}0 \\
0 \\
0\end{array}$ \\
\hline \multirow{5}{*}{ 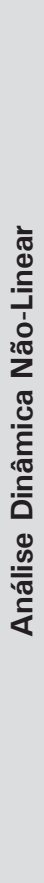 } & \multirow{5}{*}{ 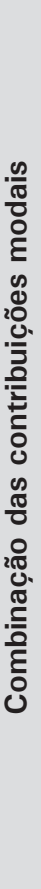 } & $\begin{array}{l}\text { م) } \\
\text { ס }\end{array}$ & $\sum_{\underline{\underline{\Sigma}}}^{\underline{\underline{\varepsilon}}}$ & $\begin{array}{l}8 \\
\text { ○ } \\
\end{array}$ & $\bar{m}$ & $\begin{array}{l}0 \\
\bullet \\
\sigma\end{array}$ & $\frac{\delta}{\delta}$ & 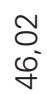 & $\frac{8}{6}$ & $\begin{array}{l}0 \\
\text { N } \\
\text { N }\end{array}$ \\
\hline & & $\begin{array}{l}\dot{\sigma} \\
\sigma \\
\sigma\end{array}$ & $\begin{array}{l}\bar{E} \\
\text { Ė } \\
\text { In }\end{array}$ & $\begin{array}{l}\text { ○ } \\
\text { ○' }\end{array}$ & $\begin{array}{l}\bar{m} \\
\bar{\sigma}\end{array}$ & $\begin{array}{l}0 \\
\mathscr{Q} \\
\text { "ే }\end{array}$ & $\frac{5}{\dot{m}}$ & 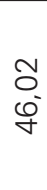 & $\begin{array}{l}\text { Q } \\
\text { চ் }\end{array}$ & 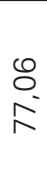 \\
\hline & & $\begin{array}{l}m \\
\text { ๘ } \\
\sigma\end{array}$ & $\begin{array}{l}\bar{\xi} \\
\underline{\underline{E}}\end{array}$ & $\begin{array}{l}8 \\
0 \\
0\end{array}$ & $\bar{n}$ & 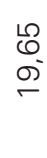 & $\begin{array}{l}\text { ○. } \\
\text { m }\end{array}$ & $\begin{array}{l}5 \\
0 \\
0 \\
\end{array}$ & 占 & $\begin{array}{l}0 \\
0 \\
N\end{array}$ \\
\hline & & $\begin{array}{c}N \\
0 \\
\sigma\end{array}$ & $\begin{array}{l}\overline{\underline{E}} \\
\underline{\underline{z}}\end{array}$ & $\begin{array}{l}8 \\
0 \\
0\end{array}$ & $\begin{array}{l}\text { m. } \\
\text { "i }\end{array}$ & 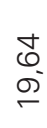 & 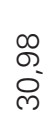 & $\begin{array}{l}8 \\
0 \\
0 \\
+\end{array}$ & $\begin{array}{l}\text { Jా } \\
\bar{\sigma}\end{array}$ & $\begin{array}{l}\text { 오 } \\
\text { N }\end{array}$ \\
\hline & & $\begin{array}{l}r \\
\frac{0}{0} \\
\Sigma \\
\Sigma\end{array}$ & $\begin{array}{l}\overline{\underline{E}} \\
\underline{\underline{z}}\end{array}$ & $\begin{array}{l}8 \\
0 \\
0\end{array}$ & $\begin{array}{l}\text { N } \\
\text { á }\end{array}$ & $\begin{array}{l}\bar{\sigma} \\
\sigma \\
\sigma\end{array}$ & $\begin{array}{l}\text { m. } \\
\text { ' } \\
\text { m. }\end{array}$ & 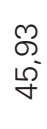 & $\begin{array}{l}0 \\
\infty \\
\bar{\sigma}\end{array}$ & 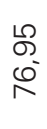 \\
\hline \multicolumn{2}{|c|}{ 完 } & & ${ }_{\underline{\underline{E}}}^{\bar{E}}$ & ০০ & 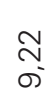 & $\begin{array}{l}\stackrel{+}{+} \\
\stackrel{-}{-}\end{array}$ & $\begin{array}{l}\stackrel{P}{0} \\
\text { о }\end{array}$ & 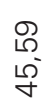 & $\begin{array}{l}\text { ণ } \\
\dot{\sigma}\end{array}$ & $\begin{array}{l}\infty \\
\prod^{\infty} \\
\omega^{\prime}\end{array}$ \\
\hline 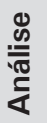 & 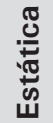 & & $\begin{array}{l}\overline{\underline{E}} \\
\text { 兰 }\end{array}$ & $\begin{array}{l}8 \\
0 \\
0\end{array}$ & $\bar{\sigma}$ & $\begin{array}{l}\llcorner \\
\varrho \\
\sigma\end{array}$ & $\stackrel{m}{\sigma}$ & ָ̊ & $\begin{array}{l}m \\
m \\
m \\
m\end{array}$ & $\begin{array}{l}\text { L } \\
0 \\
0 \\
\text { ○ }\end{array}$ \\
\hline & $N$ & & $\underline{\xi}$ & ०े & $\begin{array}{l}\text { ○ } \\
\text { হ }\end{array}$ & $\begin{array}{l}\text { O } \\
\text { ó } \\
\text { v }\end{array}$ & $\begin{array}{l}\text { O } \\
\text { N }\end{array}$ & 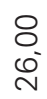 & $\begin{array}{l}\circ \\
\stackrel{\circ}{\circ} \\
\stackrel{0}{0}\end{array}$ & $\begin{array}{l}\stackrel{0}{-} \\
\underset{J}{J}\end{array}$ \\
\hline
\end{tabular}




\begin{tabular}{|c|c|c|c|c|c|c|c|c|c|c|c|}
\hline \multicolumn{2}{|c|}{$\begin{array}{l}\text { 之 } \\
\text { 岁 } \\
\text { 字 }\end{array}$} & & $\begin{array}{l}\overline{\underline{\xi}} \\
\underline{\underline{\sum}}\end{array}$ & $\frac{\stackrel{L}{N}}{\infty}$ & $\begin{array}{l}\stackrel{+}{N} \\
\stackrel{0}{0} \\
\stackrel{O}{-}\end{array}$ & $\begin{array}{l}\stackrel{D}{N} \\
\stackrel{0}{\sim}\end{array}$ & $\begin{array}{l}\bar{\sigma} \\
\sigma^{-} \\
\square\end{array}$ & $\begin{array}{l}0 \\
0 \\
0\end{array}$ & $\begin{array}{l}\text { g } \\
\text { m } \\
\text { g- }\end{array}$ & 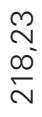 & $\begin{array}{l}\stackrel{N}{N} \\
\stackrel{N}{N}\end{array}$ \\
\hline \multicolumn{2}{|c|}{ 㟔 } & & $\begin{array}{l}\bar{E} \\
\underline{\underline{E}}\end{array}$ & $\begin{array}{l}0 \\
0 \\
0 \\
\infty\end{array}$ & $\begin{array}{l}\text { m } \\
0 \\
0\end{array}$ & $\begin{array}{l}\tilde{m} \\
\stackrel{\Xi}{ \pm} \\
\leftarrow\end{array}$ & 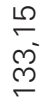 & 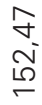 & 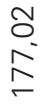 & 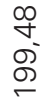 & $\begin{array}{l}\text { న } \\
\text { D } \\
\text { N }\end{array}$ \\
\hline \multirow{5}{*}{ 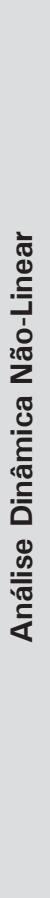 } & \multirow{5}{*}{ 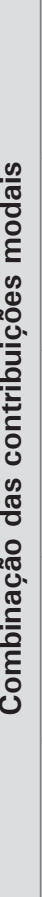 } & $\begin{array}{l}\text { ס } \\
\sigma \\
\sigma\end{array}$ & $\begin{array}{l}\overline{\underline{\xi}} \\
\underline{\underline{\underline{z}}}\end{array}$ & $\begin{array}{l}\text { నొ } \\
\text { ద' } \\
\infty\end{array}$ & $\begin{array}{l}\widetilde{0} \\
0^{\circ} \\
\text { बै }\end{array}$ & $\begin{array}{l}\underset{\sim}{\sigma} \\
\check{\Sigma} \\
\leftarrow\end{array}$ & \begin{tabular}{l}
$\infty$ \\
0 \\
\multirow{1}{-}{} \\
m.
\end{tabular} & $\begin{array}{l}\widetilde{N} \\
\infty \\
\stackrel{\sim}{0} \\
\sim\end{array}$ & $\begin{array}{l}\stackrel{\llcorner}{N} \\
\stackrel{\infty}{\infty}\end{array}$ & $\begin{array}{l}\overline{0} \\
\text { L } \\
0\end{array}$ & $\begin{array}{l}O \\
\check{\Sigma} \\
\bar{N}\end{array}$ \\
\hline & & $\begin{array}{l}+ \\
\sigma \\
\sigma\end{array}$ & $\begin{array}{l}\overline{\underline{\xi}} \\
\underline{\underline{\underline{\nu}}}\end{array}$ & 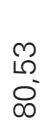 & $\begin{array}{l}\widetilde{0} \\
\widetilde{\sigma} \\
\sigma\end{array}$ & 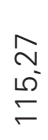 & 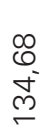 & \begin{tabular}{l}
$N$ \\
$\infty$ \\
\multirow{L}{L}{} \\
$\sim$
\end{tabular} & $\stackrel{\stackrel{L}{N}}{\underset{\infty}{\infty}}$ & $\begin{array}{l}\overline{0} \\
\stackrel{0}{0} \\
\sim\end{array}$ & $\frac{O}{\bar{E}}$ \\
\hline & & $\begin{array}{l}m \\
\sigma \\
\sigma\end{array}$ & $\begin{array}{l}\overline{\underline{E}} \\
\underline{\underline{z}}\end{array}$ & $\begin{array}{l}\text { กิ } \\
\infty \\
\infty\end{array}$ & $\begin{array}{l}\widetilde{N} \\
\mathscr{6} \\
\mathscr{8}\end{array}$ & 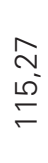 & \begin{tabular}{l}
$\infty$ \\
0 \\
\multirow{1}{*}{} \\
m
\end{tabular} & 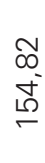 & $\stackrel{\stackrel{L}{\sim}}{\stackrel{\infty}{\infty}}$ & $\begin{array}{l}\overline{0} \\
\text { - } \\
\text { N }\end{array}$ & 듣 \\
\hline & & $\begin{array}{l}N \\
0 \\
-\end{array}$ & $\begin{array}{l}\overline{\underline{\xi}} \\
\underline{\underline{z}}\end{array}$ & $\begin{array}{l}\overline{1} \\
0 \\
\infty\end{array}$ & $\begin{array}{l}\overline{0} \\
\varnothing \\
\mathscr{0}\end{array}$ & $\begin{array}{l}\stackrel{0}{N} \\
\stackrel{\sigma}{\sigma} \\
\leftarrow\end{array}$ & $\begin{array}{l}0 \\
0 \\
\text { ले } \\
\text { - }\end{array}$ & $\begin{array}{l}\bar{\infty} \\
\tilde{\sigma} \\
{[}\end{array}$ & $\begin{array}{l}\stackrel{10}{N} \\
\stackrel{\infty}{\infty}\end{array}$ & $\begin{array}{l}\overline{6} \\
\text { ம) } \\
\text { N }\end{array}$ & $\begin{array}{l}\stackrel{0}{E} \\
\check{\tau}\end{array}$ \\
\hline & & $\begin{array}{l}- \\
\frac{0}{8} \\
\Sigma\end{array}$ & $\begin{array}{l}\overline{\underline{\varepsilon}} \\
\underline{\underline{\underline{z}}}\end{array}$ & $\begin{array}{l}\underset{\checkmark}{\checkmark} \\
\stackrel{\circ}{\infty}\end{array}$ & $\begin{array}{l}\circ \\
\text { டீ } \\
\text { बீ }\end{array}$ & $\begin{array}{l} \pm \\
\stackrel{5}{5} \\
\leftarrow\end{array}$ & \begin{tabular}{l}
$\stackrel{\llcorner}{\llcorner}$ \\
\multirow{2}{*}{} \\
m
\end{tabular} & 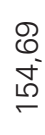 & 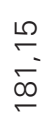 & 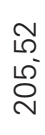 & $\underset{\widetilde{O}}{\check{\sigma}}$ \\
\hline \multicolumn{2}{|c|}{ 定 } & & $\underline{\underline{\underline{\varepsilon}}}_{\underline{\underline{z}}}$ & $\begin{array}{l}\text { N } \\
\text { న } \\
\end{array}$ & $\frac{\mathscr{R}}{\stackrel{0}{\circ}}$ & $\begin{array}{l}\stackrel{ }{m} \\
\underset{\leftarrow}{+} \\
\leftarrow\end{array}$ & $\begin{array}{l}\text { Ln } \\
\text { m } \\
\text { m }\end{array}$ & 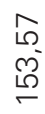 & 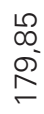 & 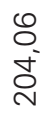 & $\begin{array}{l}\tilde{N} \\
\text { D' } \\
\text { న }\end{array}$ \\
\hline 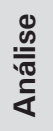 & 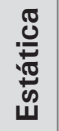 & & $\begin{array}{l}\overline{\underline{\varepsilon}} \\
\underline{\underline{\underline{z}}}\end{array}$ & $\begin{array}{l}\mathscr{0} \\
\stackrel{\sim}{~} \\
\stackrel{Y}{*}\end{array}$ & 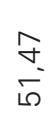 & 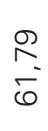 & $\begin{array}{l}0 \\
\stackrel{1}{N} \\
N\end{array}$ & $\hat{\bar{N}}$ & $\begin{array}{l}\text { ¿ } \\
8 \\
8 \\
0\end{array}$ & $\begin{array}{l}\hat{0} \\
\sigma \\
\sigma \\
\sigma\end{array}$ & $\begin{array}{l}\text { f } \\
\infty \\
\infty^{\circ} \\
F\end{array}$ \\
\hline & $N$ & & छ & $\begin{array}{l}\text { 尺) } \\
\text { ָे }\end{array}$ & $\begin{array}{l}\text { O } \\
\text { Nं }\end{array}$ & $\begin{array}{l}\bigcirc \\
\text { ํ }\end{array}$ & $\begin{array}{l}\text { ○ } \\
\text { స }\end{array}$ & $\begin{array}{l}\text { O } \\
\text { O } \\
\text { ○ }\end{array}$ & $\begin{array}{l}\text { ㅇ } \\
\text { த) }\end{array}$ & $\begin{array}{l}0 \\
\infty \\
\infty\end{array}$ & $\begin{array}{l}\text { ㅇ } \\
\text { - }\end{array}$ \\
\hline
\end{tabular}




\begin{tabular}{|c|c|c|c|c|c|c|c|c|c|c|c|}
\hline \multicolumn{2}{|c|}{$\begin{array}{l}\text { ह } \\
\text { के } \\
\text { 宅 }\end{array}$} & & $\underline{\underline{\underline{\varepsilon}}}$ & $\begin{array}{l}\stackrel{ }{N} \\
\infty 0^{-} \\
\underset{\sim}{+}\end{array}$ & 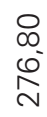 & $\begin{array}{l}\underset{N}{N} \\
\stackrel{n}{O}\end{array}$ & $\begin{array}{l}\text { D } \\
\text { mं } \\
\text { m }\end{array}$ & $\begin{array}{l}\text { J } \\
\text { N } \\
0 \\
\text { ले }\end{array}$ & $\begin{array}{l}\text { N } \\
\text { D } \\
\infty \\
\text { లn }\end{array}$ & $\begin{array}{l}\text { व } \\
\text { ம் } \\
\text { ల }\end{array}$ & 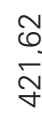 \\
\hline $\bar{c}$ & & & $\sum_{\underline{\underline{E}}}^{\overline{\underline{E}}}$ & $\begin{array}{l}\text { I } \\
\text { N }\end{array}$ & $\begin{array}{l}\stackrel{\llcorner}{m} \\
\stackrel{\mathrm{N}}{\mathrm{N}}\end{array}$ & $\begin{array}{l}\text { L } \\
\text { D' } \\
\stackrel{2}{N}\end{array}$ & $\begin{array}{l}\overline{0} \\
0 \\
0 \\
\text { ల) }\end{array}$ & $\begin{array}{l}\text { N } \\
\text { N } \\
\text { m }\end{array}$ & 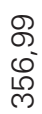 & $\begin{array}{l}\bar{d} \\
\text { v } \\
\text { ల }\end{array}$ & $\begin{array}{l}\text { m. } \\
\infty \\
\infty \\
\infty\end{array}$ \\
\hline \multirow{5}{*}{ 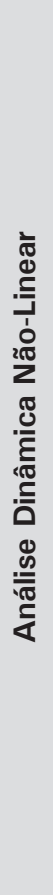 } & \multirow{5}{*}{ 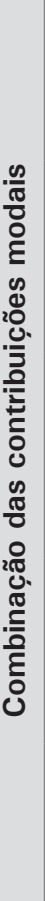 } & $\begin{array}{l}0 \\
\sigma \\
\sigma\end{array}$ & $\sum_{\underline{\underline{E}}}^{\bar{\xi}}$ & $\begin{array}{l}\infty \\
\underset{\sigma}{0} \\
\stackrel{N}{N}\end{array}$ & 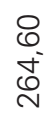 & $\begin{array}{l}\stackrel{\llcorner}{\circ} \\
\text { N } \\
\text { N }\end{array}$ & $\begin{array}{l}\overline{0} \\
\text { స్ } \\
\text { m }\end{array}$ & $\begin{array}{l}m \\
\text { D } \\
\text { N } \\
\text { men }\end{array}$ & $\begin{array}{l}\text { Na } \\
\text { - } \\
\infty \\
\text { m. }\end{array}$ & $\begin{array}{l}\stackrel{1}{m} \\
\text { 心 } \\
\infty \\
m\end{array}$ & $\begin{array}{l}\sigma \\
\stackrel{\sigma}{\sigma}\end{array}$ \\
\hline & & $\begin{array}{l}\nabla \\
\sigma \\
\sigma\end{array}$ & $\sum_{\underline{\underline{E}}}^{\bar{\xi}}$ & $\begin{array}{l}\infty \\
\underset{\infty}{\infty} \\
\stackrel{\infty}{N}\end{array}$ & 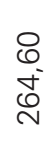 & $\begin{array}{l}\stackrel{L}{\circ} \\
\text { N } \\
\stackrel{N}{N}\end{array}$ & $\begin{array}{l}\overline{0} \\
\text { స్} \\
\text { }\end{array}$ & $\begin{array}{l}m \\
\infty \\
N \\
\tilde{n}\end{array}$ & 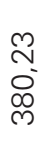 & $\begin{array}{l}\stackrel{1}{m} \\
m \\
0 \\
0 \\
m\end{array}$ & $\begin{array}{l}\underset{\sigma}{\sigma} \\
\stackrel{+}{\sigma}\end{array}$ \\
\hline & & $\begin{array}{l}m \\
\sigma \\
\sigma\end{array}$ & $\sum_{\underline{\underline{E}}}^{\bar{\xi}}$ & 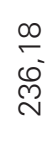 & 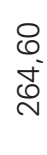 & $\begin{array}{l}\stackrel{L}{\llcorner} \\
\stackrel{\text { N}}{\text { N }}\end{array}$ & 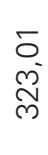 & $\begin{array}{l}m \\
\text { ñ } \\
\text { N } \\
\text { man }\end{array}$ & $\begin{array}{l}\text { N } \\
\text { O } \\
\infty \\
\text { N }\end{array}$ & $\begin{array}{l}\omega^{m} \\
0^{\circ} \\
\infty \\
m\end{array}$ & \begin{tabular}{l}
6 \\
\multirow{\sigma}{\sigma}{} \\
\multirow{\sigma}{*}{}
\end{tabular} \\
\hline & & $\begin{array}{l}N \\
0 \\
-\end{array}$ & $\bar{\sum}_{\underline{\underline{E}}}^{\bar{\varepsilon}}$ & $\begin{array}{l}\stackrel{\infty}{c} \\
\stackrel{0}{N}\end{array}$ & 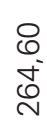 & $\begin{array}{l}\stackrel{L}{\llcorner} \\
\stackrel{N}{N} \\
\stackrel{N}{N}\end{array}$ & $\begin{array}{l}\stackrel{8}{0} \\
\text { Nं } \\
\text { N }\end{array}$ & $\begin{array}{l}\text { N } \\
\text { N } \\
\stackrel{\text { N }}{\text { N }}\end{array}$ & $\begin{array}{l}\underset{N}{N} \\
\stackrel{\infty}{\infty} \\
\text { లn }\end{array}$ & 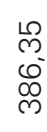 & 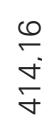 \\
\hline & & $\begin{array}{l}\text { 음 } \\
\frac{0}{2}\end{array}$ & $\bar{\sum}_{\underline{\underline{E}}}^{\bar{\varepsilon}}$ & $\begin{array}{l}\stackrel{2}{*} \\
\stackrel{\sim}{\sim}\end{array}$ & 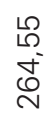 & $\begin{array}{l}\bar{\Sigma} \\
\text { N } \\
\text { N }\end{array}$ & 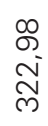 & $\begin{array}{l}\bar{\sigma} \\
\text { N } \\
\text { లn }\end{array}$ & $\begin{array}{l}\text { N } \\
\text { O } \\
\infty \\
m\end{array}$ & $\begin{array}{l}\text { Ð } \\
\text { 心్ } \\
\infty \\
\infty\end{array}$ & $\stackrel{\llcorner}{\underset{\sigma}{\sigma}}$ \\
\hline \multicolumn{2}{|c|}{ 定 } & & $\sum_{\underline{\underline{E}}}^{\bar{\varepsilon}}$ & $\begin{array}{l}\stackrel{L}{\sim} \\
\stackrel{\sim}{N}\end{array}$ & $\begin{array}{l}\stackrel{0}{O} \\
\stackrel{\text { N }}{\mathcal{N}}\end{array}$ & $\begin{array}{l}\text { D } \\
\text { চ }\end{array}$ & $\begin{array}{l}\stackrel{L}{N} \\
\stackrel{0}{N} \\
\stackrel{n}{n}\end{array}$ & $\begin{array}{l}\text { প } \\
\text { ○े } \\
\stackrel{1}{n}\end{array}$ & $\frac{N}{\hat{N}}$ & $\begin{array}{l}\text { ז } \\
\text { mं } \\
\infty \\
\text { m }\end{array}$ & $\begin{array}{l}\stackrel{1}{\sigma} \\
\text { б }\end{array}$ \\
\hline 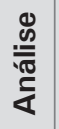 & 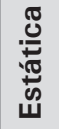 & & $\bar{\sum}_{\underline{\underline{E}}}$ & \begin{tabular}{l}
$\infty$ \\
0 \\
\multirow{2}{*}{} \\
$\stackrel{n}{-}$
\end{tabular} & $\begin{array}{l}\infty \\
\infty \\
\Sigma \\
\Sigma\end{array}$ & $\begin{array}{l}\infty \\
0 \\
0 \\
\\
-\end{array}$ & \begin{tabular}{l}
\multirow{J}{*}{} \\
$\infty 0^{-}$ \\
$\infty$ \\
-
\end{tabular} & $\begin{array}{l}\stackrel{\llcorner}{\infty} \\
\stackrel{\infty}{\circ} \\
\stackrel{\circ}{N}\end{array}$ & 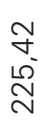 & $\begin{array}{l}\text { D } \\
\text { న్ } \\
\text { N }\end{array}$ & ָ̊ \\
\hline & $\mathbf{N}$ & & छ & $\begin{array}{l}\circ \\
\text { ㅇ } \\
-\end{array}$ & $\begin{array}{l}8 \\
0 \\
0\end{array}$ & $\begin{array}{l}\text { ㅇ } \\
\stackrel{\circ}{\circ}\end{array}$ & \begin{tabular}{l}
8 \\
○ \\
\multirow{\leftarrow}{*}{}
\end{tabular} & $\begin{array}{l}\text { ○ } \\
\text { n' }\end{array}$ & $\stackrel{0}{\sim}$ & $\begin{array}{l}\text { 尺 } \\
\text { E }\end{array}$ & $\begin{array}{l}8 \\
= \\
=\end{array}$ \\
\hline
\end{tabular}




\begin{tabular}{|c|c|c|c|c|c|c|c|c|c|c|c|}
\hline \multicolumn{2}{|c|}{$\begin{array}{l}\text { 岁 } \\
\text { 足 }\end{array}$} & & $\sum_{\underline{\underline{\Sigma}}}^{\overline{\underline{\varepsilon}}}$ & $\frac{\stackrel{\llcorner}{\sim}}{\stackrel{\leftarrow}{q}}$ & 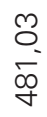 & $\begin{array}{l}\bar{\sigma} \\
\overline{0} \\
\bar{\kappa}\end{array}$ & \begin{tabular}{l} 
ऽ \\
$\infty$ \\
0 \\
\multirow{+}{0}{}
\end{tabular} & $\begin{array}{l}m \\
0 \\
0 \\
ద\end{array}$ & 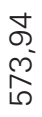 & $\begin{array}{l}\text { L } \\
\text { - } \\
0\end{array}$ & 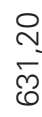 \\
\hline \multicolumn{2}{|c|}{ 峁 } & & $\begin{array}{l}\overline{\underline{\varepsilon}} \\
\underline{\underline{z}}\end{array}$ & $\begin{array}{l}\stackrel{m}{m} \\
\underset{\forall}{+}\end{array}$ & $\begin{array}{l}\infty \\
\infty \\
\tilde{J} \\
\dot{J}\end{array}$ & $\begin{array}{l}0 \\
\llcorner \\
8 \\
0 \\
\varnothing\end{array}$ & 㐫 & 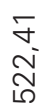 & $\begin{array}{l}\text { ब } \\
\text { స̃ } \\
\text { దூ }\end{array}$ & 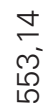 & 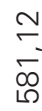 \\
\hline \multirow{5}{*}{ 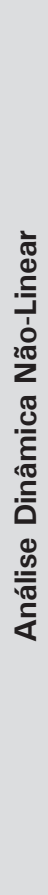 } & \multirow{5}{*}{ 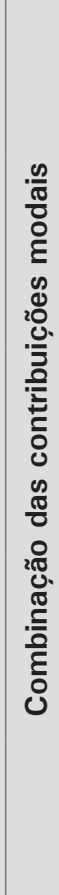 } & $\begin{array}{l}\text { L) } \\
\text { ס } \\
\end{array}$ & $\sum_{\underline{\underline{\Sigma}}}^{\overline{\underline{\varepsilon}}}$ & 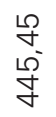 & $\stackrel{\circ}{\stackrel{\circ}{*}}$ & $\begin{array}{l}\text { の } \\
\circ \\
8 \\
\circ\end{array}$ & 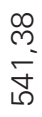 & $\begin{array}{l}\hat{0} \\
0 \\
0 \\
0\end{array}$ & $\stackrel{N}{N}$ & $\begin{array}{l}N \\
\infty \\
0 \\
0 \\
0\end{array}$ & 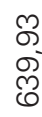 \\
\hline & & $\begin{array}{l}+ \\
\text { व } \\
-\end{array}$ & $\begin{array}{l}\overline{\underline{\xi}} \\
\sum_{\underline{\Sigma}}\end{array}$ & \begin{tabular}{l}
$\stackrel{L}{+}$ \\
$\stackrel{5}{f}$ \\
\multirow{+}{*}{}
\end{tabular} & $\stackrel{\circ}{\stackrel{\circ}{*}}$ & $\begin{array}{l}\text { O } \\
\text { த } \\
0 \\
0\end{array}$ & 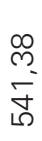 & 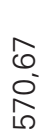 & $\frac{N}{N}$ & $\begin{array}{l}N \\
\infty \\
0 \\
8 \\
0\end{array}$ & $\begin{array}{l}\text { న } \\
\text { गे } \\
\text { రె }\end{array}$ \\
\hline & & $\begin{array}{l}m \\
\sigma \\
\sigma\end{array}$ & $\begin{array}{l}\overline{\underline{\varepsilon}} \\
\underline{\underline{z}}\end{array}$ & 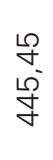 & $\frac{\circ}{\stackrel{2}{~}}$ & 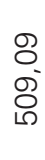 & 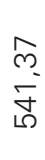 & $\begin{array}{l}0 \\
0 \\
0 \\
\text { م. }\end{array}$ & $\frac{N}{N}$ & $\begin{array}{l}N \\
\infty \\
0 \\
8 \\
0\end{array}$ & 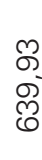 \\
\hline & & $\begin{array}{c}N \\
0 \\
-\end{array}$ & $\sum_{\underline{\underline{E}}}^{\overline{\underline{E}}}$ & 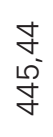 & $\stackrel{\circ}{\stackrel{\circ}{\sim}}$ & $\begin{array}{l}\infty \\
\circ \\
0 \\
\text { D } \\
\circ\end{array}$ & 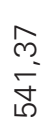 & $\begin{array}{l}\hat{0} \\
0 \\
0 \\
0\end{array}$ & 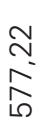 & $\begin{array}{l}\stackrel{1}{0} \\
0 \\
0 \\
0\end{array}$ & $\begin{array}{l}\text { ñ } \\
\text { లో }\end{array}$ \\
\hline & & $\frac{r}{\frac{0}{0}}$ & $\begin{array}{l}\underline{\underline{\varepsilon}} \\
\underline{\underline{z}}\end{array}$ & $\begin{array}{l}\text { 寸 } \\
\stackrel{+}{+}\end{array}$ & $\stackrel{\circ}{\stackrel{ }{*}}$ & $\begin{array}{l}\infty \\
0 \\
0 \\
0 \\
0\end{array}$ & ڤ̊ & $\begin{array}{l}0 \\
0 \\
0 \\
0 \\
1\end{array}$ & $\underset{ }{\overleftarrow{N}}$ & $\begin{array}{l}\circ \\
\infty \\
0 \\
0 \\
0\end{array}$ & 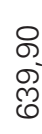 \\
\hline \multicolumn{2}{|c|}{ 定 } & & $\sum_{\underline{\underline{E}}}^{\overline{\underline{E}}}$ & 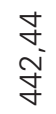 & \begin{tabular}{l}
$\infty$ \\
$\infty$ \\
$\infty$ \\
\multirow{\sigma}{*}{}
\end{tabular} & $\begin{array}{l}\infty \\
0 \\
10 \\
0 \\
0\end{array}$ & $\begin{array}{r}\hat{N} \\
\hat{n}\end{array}$ & $\begin{array}{l}\infty \\
\infty \\
0 \\
0 \\
0 \\
1\end{array}$ & 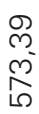 & 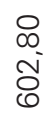 & $\begin{array}{c}\stackrel{0}{1} \\
\text { లో }\end{array}$ \\
\hline 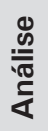 & 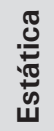 & & $\begin{array}{l}\overline{\underline{\varepsilon}} \\
\underline{\underline{z}}\end{array}$ & 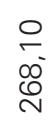 & $\begin{array}{l}\bar{\sigma} \\
\stackrel{\infty}{\infty} \\
\stackrel{\infty}{N}\end{array}$ & $\begin{array}{l}\text { L' } \\
\text { o } \\
\text { m }\end{array}$ & $\begin{array}{l}\text { m } \\
\stackrel{N}{N} \\
\tilde{m}\end{array}$ & 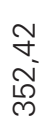 & 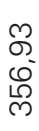 & \begin{tabular}{l}
\multirow{f}{*}{} \\
$\stackrel{f}{\text { s }}$
\end{tabular} & $\begin{array}{l}\infty \\
0 \\
8 \\
8 \\
\forall\end{array}$ \\
\hline & $N$ & & $\widehat{\underline{\xi}}$ & $\begin{array}{l}8 \\
\circ \\
\circ\end{array}$ & $\begin{array}{l}\circ \\
\text { - }\end{array}$ & $\infty^{\circ}$ & $\stackrel{\circ}{\circ}$ & $\frac{0}{\sigma^{\circ}}$ & $\begin{array}{l}8 \\
\text { வ }\end{array}$ & $\begin{array}{l}8 \\
8 \\
10\end{array}$ & ০ \\
\hline
\end{tabular}




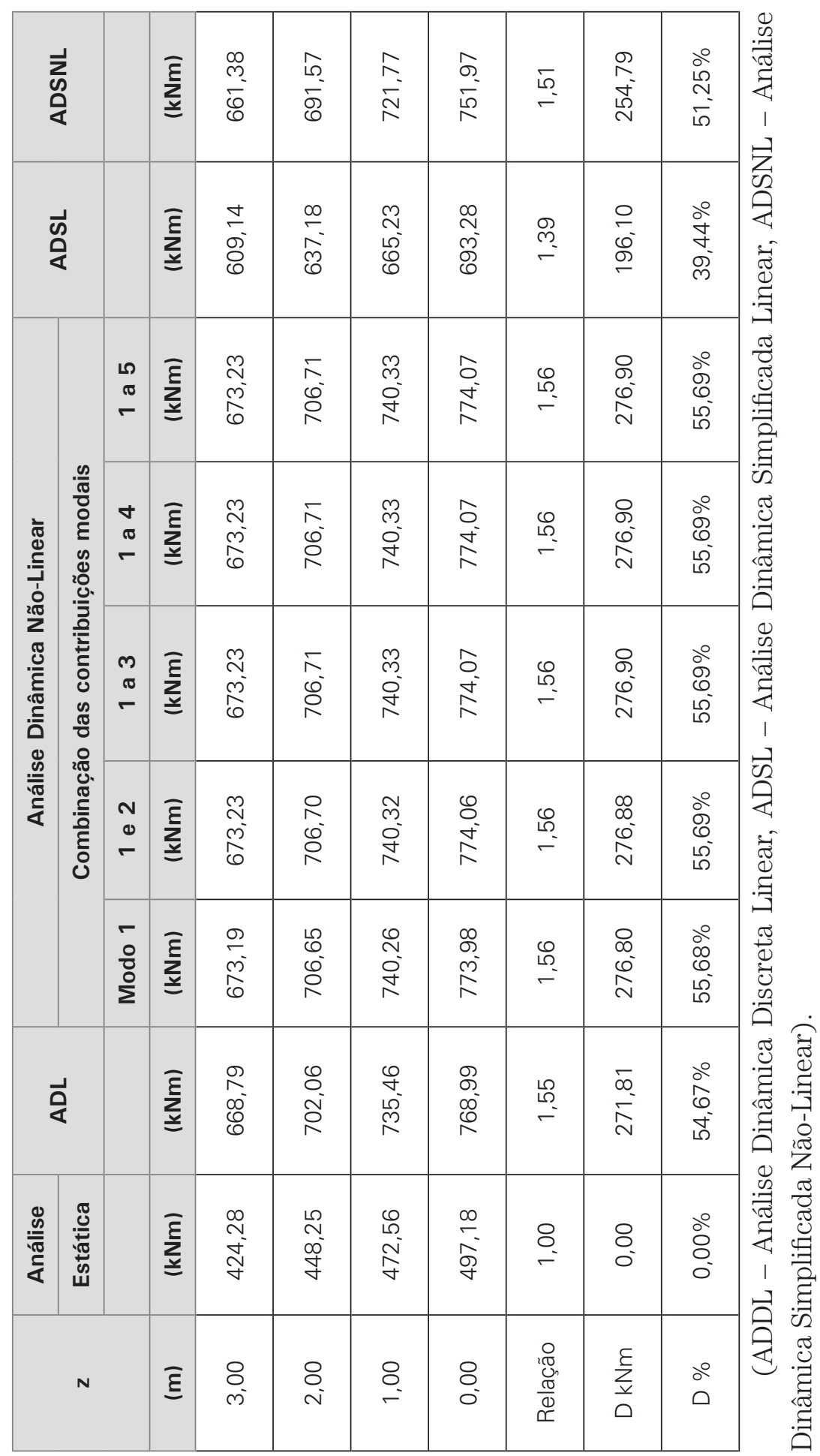


A Figura 7.41 condensa e apresenta os resultados obtidos das análises considerando a ação do vento sobre a estrutura, onde, de baixo para cima, estão, respectivamente, a análise estática; as análises dinâmicas simplificadas, linear e não-linear; e as análises dinâmicas discretas, linear e não-linear do $1^{\circ}$ modo e modos superiores até o $5^{\circ}$, respectivamente.

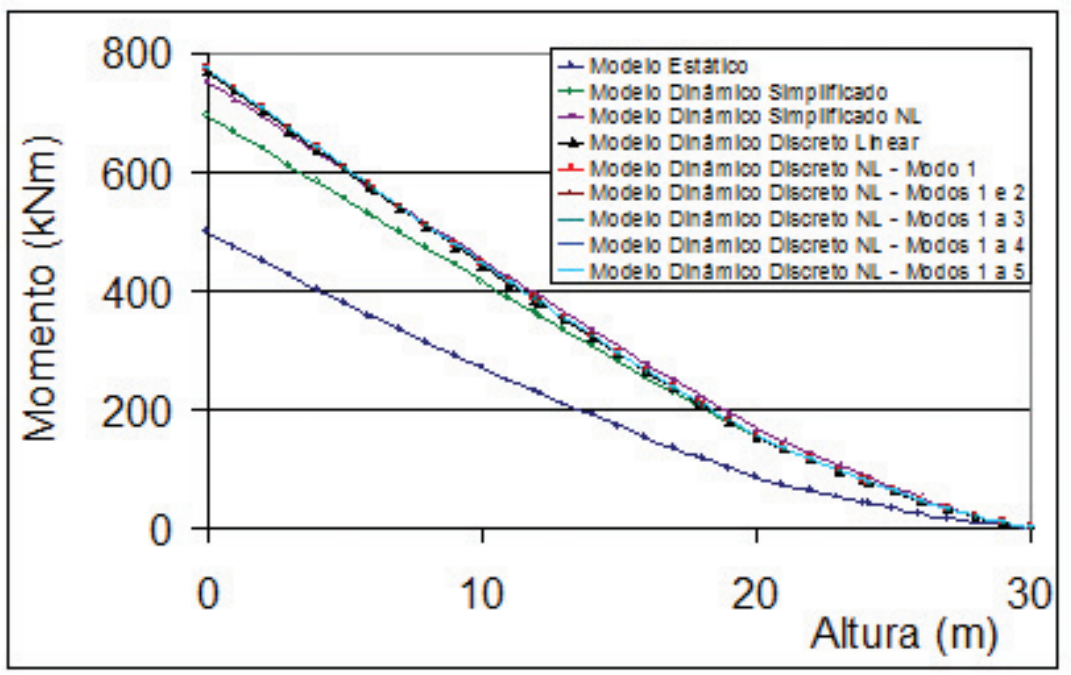

Figura 7.41 - Ação do vento na estrutura 5.

As formas modais da NBR 6123/88, a do Método dos Elementos Finitos e a do método proposto constam na Figura 7.42(a).

Uma melhor aproximação à forma modal não-linear é conseguida substituindo-se o expoente da expressão (6.6) por 1,85; tendo em vista que a curva definida com esse novo expoente oferece uma menor diferença entre seus pontos e os da curva da forma modal não-linear, como pode ser observado na Figura 7.42(b).

O expoente sugerido anteriormente distancia-se $9 \%$ do valor do recomendado pela NBR 6123/88. 


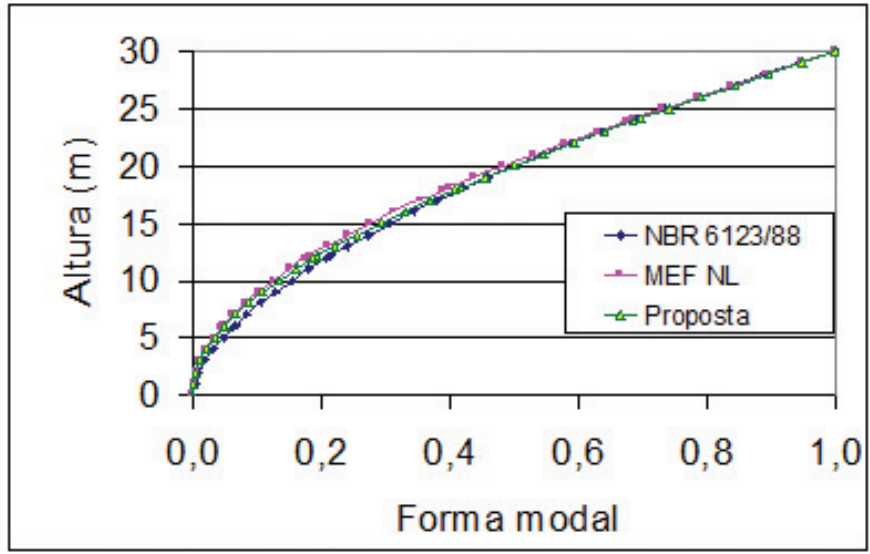

(a) expoente da forma modal: $\gamma=1,7$

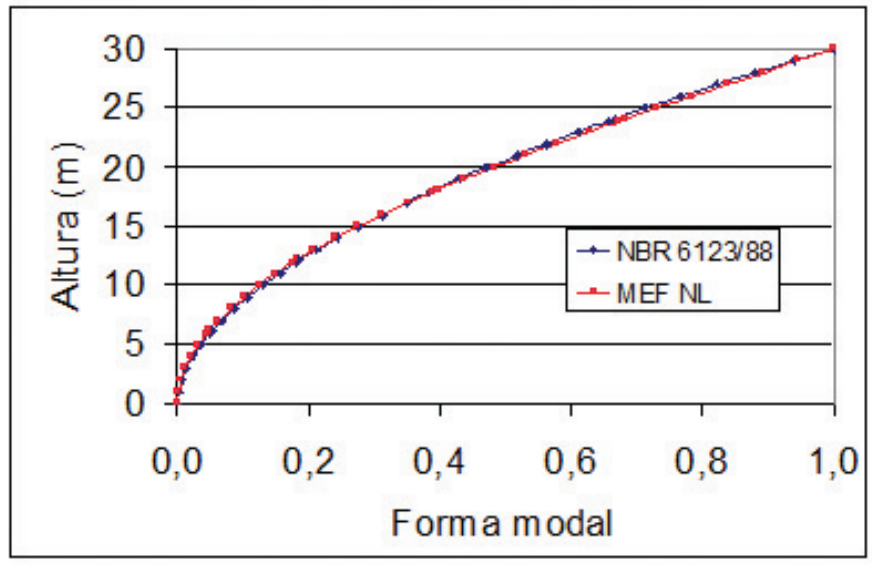

(b) expoente da forma modal: $\gamma=1,85$

Figura 7.42 - Estrutura 5 - comparativo das formas modais: (a) usada na análise, (b) sugerida.

\subsection{RESUMO}

A Tabela 7.30 resume as frequências naturais das estruturas analisadas. Os fatores que nela são indicados se referem à relação entre os resultados da aplicação direta das Eq. (4.19) ou (4.21), denominada de solução aproximada do método proposto, com a solução completa do método e com o Método dos Elementos Finitos. 


\begin{tabular}{|c|c|c|c|c|c|c|c|}
\hline$\frac{\propto}{\frac{\alpha}{z}}$ & $\begin{array}{l}\infty \\
\infty \\
\stackrel{\infty}{m} \\
\underset{\sigma}{\sigma}\end{array}$ & $\begin{array}{l}\text { D } \\
\infty \\
\infty \\
\infty \\
\infty \\
\\
\end{array}$ & \begin{tabular}{l}
$\bar{\sigma}$ \\
\multirow{\sigma}{0}{} \\
ᄋ \\
-
\end{tabular} & $\begin{array}{l}\hat{\theta} \\
\mathbb{0} \\
\mathbb{0} \\
\mathbb{0} \\
\mathbb{E}\end{array}$ & $\begin{array}{l}\hat{0} \\
0 \\
0 \\
0 \\
0 \\
-\end{array}$ & $\begin{array}{l}\underset{\sim}{+} \\
\underset{\delta}{+} \\
0 \\
0\end{array}$ & \\
\hline \multirow{3}{*}{ 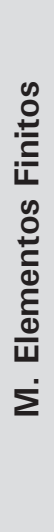 } & 흄 & 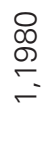 & $\begin{array}{l}\circ \\
\text { ஓ } \\
\text { న్ }\end{array}$ & 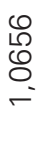 & $\begin{array}{l}\underset{c}{0} \\
\stackrel{N}{N} \\
\stackrel{2}{2}\end{array}$ & $\begin{array}{l}\stackrel{0}{ } \\
\text { Oे } \\
\text { m. }\end{array}$ & $\begin{array}{l}\stackrel{\bullet}{2} \\
\stackrel{N}{N} \\
-\end{array}$ \\
\hline & 之 & 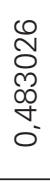 & 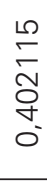 & 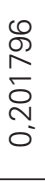 & \begin{tabular}{l}
$\stackrel{L}{\infty}$ \\
$\stackrel{N}{N}$ \\
\multirow{J}{\pm}{} \\
$\dot{0}$
\end{tabular} & 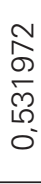 & $\begin{array}{l}\| \\
. \mathbb{0} \\
\frac{\mathbb{O}}{\mathbb{O}} \\
\Sigma\end{array}$ \\
\hline & & 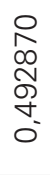 & $\begin{array}{l}\hat{0} \\
⺊ \\
0 \\
\tilde{\sigma} \\
0 \\
0\end{array}$ & 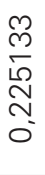 & 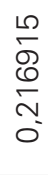 & 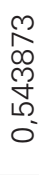 & \\
\hline \multirow{4}{*}{ 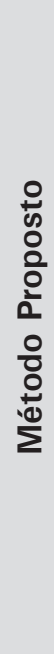 } & $\underset{\stackrel{0}{\circ}}{\stackrel{ \pm}{\leftarrow}}$ & $\begin{array}{l}\text { f } \\
\text { ग् } \\
\text { - }\end{array}$ & 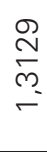 & 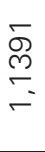 & 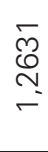 & $\begin{array}{l}\stackrel{g}{N} \\
\stackrel{m}{\sigma}\end{array}$ & $\begin{array}{l}\stackrel{L}{\llcorner} \\
\infty \\
\stackrel{\infty}{\sim} \\
-\end{array}$ \\
\hline & $\begin{array}{l}\text { 㐅̀ } \\
\frac{0}{2}\end{array}$ & $\begin{array}{l}m \\
\text { g } \\
\bar{m} \\
\text { ó } \\
0\end{array}$ & $\begin{array}{l}\infty \\
1 \\
0 \\
1 \\
0 \\
0 \\
0\end{array}$ & 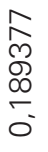 & $\begin{array}{l}0 \\
\frac{0}{N} \\
\frac{1}{\sigma} \\
\overline{0}\end{array}$ & $\begin{array}{l}\bar{\delta} \\
0 \\
\infty \\
\text { o } \\
\text { o }\end{array}$ & $\begin{array}{l}\text { II } \\
. \frac{\mathbb{O}}{0} \\
\sum^{\mathbb{E}}\end{array}$ \\
\hline & $\bar{z}$ & 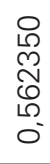 & $\begin{array}{l}\text { o } \\
\text { mे } \\
\text { ó } \\
0\end{array}$ & 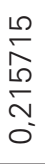 & 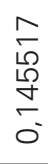 & $\begin{array}{l}\stackrel{0}{ } \\
\text { D } \\
\hat{N} \\
\stackrel{0}{\rho} \\
0^{\circ}\end{array}$ & \\
\hline & 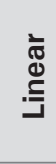 & $\begin{array}{l}\text { వ } \\
\text { D } \\
6 \\
\llcorner \\
0 \\
0\end{array}$ & $\begin{array}{l}\text { Jे } \\
\stackrel{0}{\sigma} \\
\dot{\sigma} \\
0\end{array}$ & 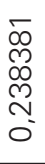 & 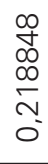 & 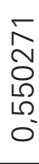 & \\
\hline \multicolumn{2}{|c|}{$-\widehat{\underline{\xi}}$} & $\stackrel{\infty}{+}$ & $\overline{6}$ & ㅇ & Q & ஓे & \multirow{3}{*}{ 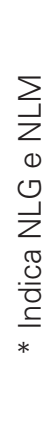 } \\
\hline \multirow[t]{2}{*}{ 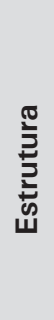 } & $\stackrel{\circ}{\gtrless}$ & 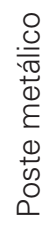 & 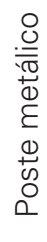 & 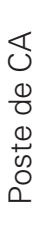 & \begin{tabular}{l}
$*$ \\
\multirow{U}{*}{} \\
0 \\
0 \\
0 \\
0 \\
0 \\
0 \\
0 \\
0
\end{tabular} & 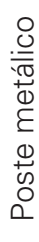 & \\
\hline & $\stackrel{\circ}{z}$ & - & $N$ & $m$ & $\checkmark$ & ما & \\
\hline
\end{tabular}


Puderam ser apreciados os expoentes $\gamma$ da forma modal

$$
\mathrm{x}=\left(\frac{\mathrm{z}}{\mathrm{h}}\right)^{\gamma}
$$

prevista na NBR 6123/88, obtendo-se valores que melhor a aproximam à forma modal dos modelos não-lineares. Os resultados estão na Tabela 7.31. Para a estrutura 4 o expoente apresentado, correspondente à forma modal da NBR 6123/88, é relativo ao cálculo, cujos resultados foram mais desfavoráveis.

Tabela 7.31 - Expoente das formas modais.

\begin{tabular}{|c|c|c|c|c|c|}
\hline \multicolumn{2}{|c|}{ Estrutura } & Altura & $\begin{array}{c}\text { Previsto na } \\
\text { NBR 6123/88 }\end{array}$ & $\begin{array}{c}\text { Sugerido } \\
\text { por esta } \\
\text { pesquisa }\end{array}$ & $\begin{array}{c}\text { Diferença (\%) } \\
\text { em relação à } \\
\text { NBR }\end{array}$ \\
\hline 1 & $\begin{array}{c}\text { Tipo } \\
\text { metálico }\end{array}$ & 48 & 1,7 & 1,965 & -16 \\
\hline 2 & $\begin{array}{c}\text { Poste } \\
\text { metálico }\end{array}$ & 61 & 1,7 & 1,775 & -4 \\
\hline 4 & $\begin{array}{c}\text { Poste de CA } \\
\text { Poste de CA }\end{array}$ & 40 & 1,7 & 1,600 & 6 \\
\hline 5 & $\begin{array}{c}\text { Poste } \\
\text { metálico }\end{array}$ & 30 & 1,7 & 1,770 & -4 \\
\hline
\end{tabular}

Para a determinação da ação do vento empregaram-se os processos normativos vigentes, assim denominados: análise estática, análise dinâmica simplificada e análise dinâmica discreta. Fez-se a introdução de mais um modelo de cálculo que ficou denominado de análise dinâmica simplificada não-linear, por estar baseada na frequência e na forma de vibração do modelo não-linear.

Foram feitas duas análises dinâmicas discretas. Uma tinha caráter puramente linear, tanto do ponto de vista geométrico quanto ma- 
terial, enquanto que a outra possuía caráter não-linear geométrico e não-linear material quando indicado. No modelo dinâmico não-linear foram computadas as contribuições do $1^{\circ}$ ao $5^{\circ}$ modo de vibração na parcela relativa às flutuações sobre a velocidade média.

Foi visto que o $1^{\circ}$ modo de vibração é o que aporta maior contribuição na resposta da estrutura dentre os outros modos de vibração. Supera, nos casos analisados, sempre a contribuição do vento médio na resposta dinâmica total.

Foram apreciadas as diferenças produzidas na determinação do momento em relação à base das estruturas pelos processos de cálculo mencionados. A Tabela 7.32 traz os momentos fletores máximos nas estruturas em relação à análise estática. Para a estrutura 4 os resultados apresentados são os referentes à taxa de amortecimento crítico de 0,01

A relação entre a as análises dinâmicas discretas pode ser vista na última coluna da Tabela 7.32. 


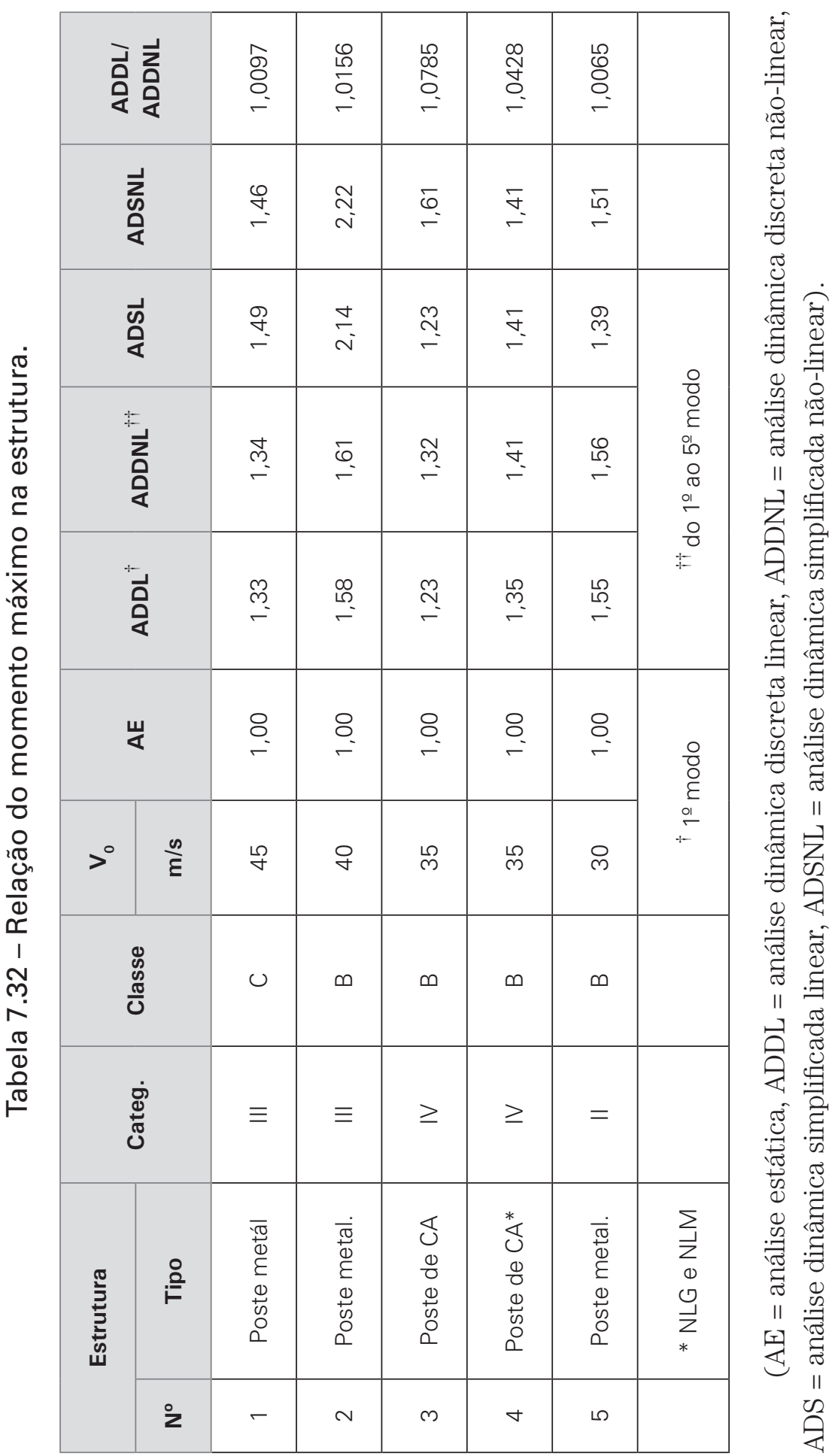


